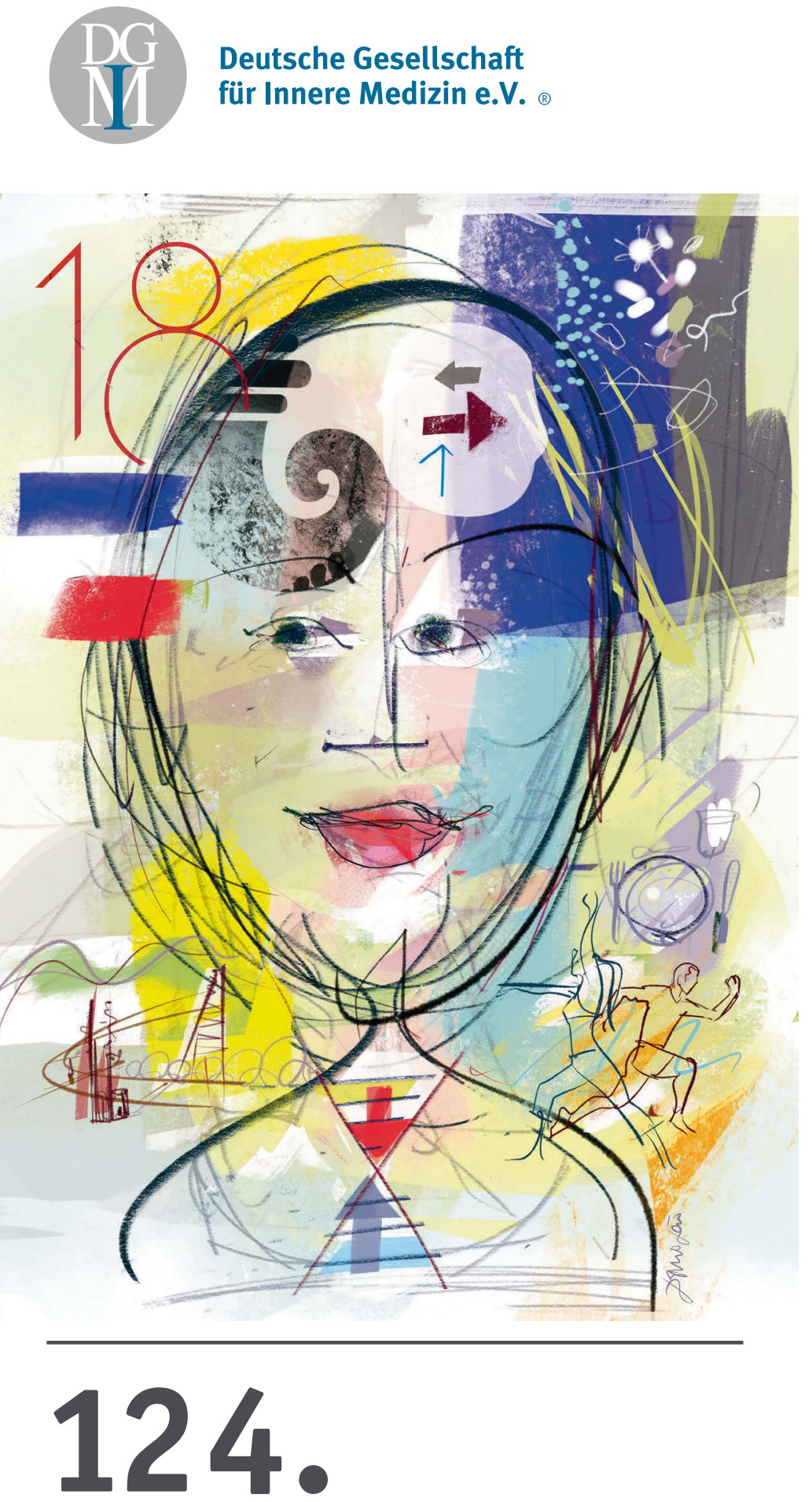

Kongress der

Deutschen Gesellschaft für Innere Medizin e.V.

Congress Center Rosengarten - Mannheim 14. - 17. April 2018

Abstractband 


\section{Kongress der Deutschen Gesellschaft für Innere Medizin e.V.}

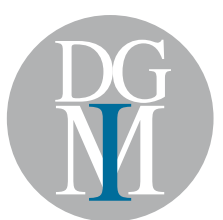

Internist $2018 \cdot 59$ (Suppl 1): S1-S82

DOI 10.1007/s00108-018-0419-y

Online publiziert: 23. März 2018

๑) Springer Medizin Verlag GmbH, ein Teil von Springer Nature 2018

\section{Wissenschaftliche Organisation}

\section{Kongress-Präsident}

Prof. Dr. med. Cornel Sieber

DGIM-Kongress-Team 2018

PD Dr. phil. Ellen Freiberger

Prof. Dr. med. Ute Hoffmann

PD Dr. med. Katrin Singler

Prof. Dr. rer. nat. Dorothee Volkert

\section{Kongress-Sekretariat}

Ulrike Freiheit

Institut für Biomedizin des Alterns

Kobergerstr. 60

90408 Nürnberg

Annemarie Volkmann

Krankenhaus Barmherzige Brüder

Prüfeninger Str. 86

93049 Regensburg
DGIM-Geschäftsstelle

DG Irenenstraße 1

65189 Wiesbaden

\section{Geschäftsführung}

RA Maximilian Broglie

Stellvertretende Geschäftsführerin

Dipl.-Kffr. Ourania Menelaou

Managementassistentin

Nicole Safenauer

Kongress-Sekretärin der DGIM

Katharina Wick

Verantwortlich für Patiententag

Dr. med. Norbert Schütz

Telefon: +49 611 - 2058040 - 0

Telefax: +49 611 - 2058040 - 46

info@dgim.de -www.dgim.de

Kongress Agentur (PCO)

m:con - mannheim:congress $\mathrm{GmbH}$

Thilo Hübner

Rosengartenplatz 2

D-68161 Mannheim

www.mcon-mannheim.de 


\section{Kongress der Deutschen Gesellschaft für Innere Medizin e.V.}

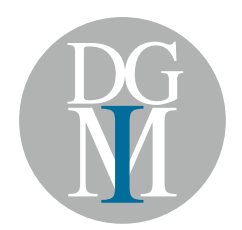

\section{Abstract-Gutachter DGIM 2018}

Altern und Altersmedizin

Simm, Andreas, Prof. Dr. med., Halle

Bauer, Jürgen, Prof. Dr. med., Heidelberg

Ernährungsmedizin / Mikrobiom

Bischoff, Stefan, Prof. Dr. med., Stuttgart

Ockenga, Johann, Prof. Dr. med., Bremen

Immunologie / Infektiologie

Müller-Ladner, Ulf, Prof. Dr. med., Bad Nauheim

Salzberger, Bernd, Prof. Dr. med., Regensburg

Innere Medizin integral

Schumm-Draeger, Petra, Prof. Dr. med., München Müller-Werdan, Ursula, Prof. Dr. med., Berlin Interdisziplinarität in der Inneren Medizin Püllen, Rupert, PD Dr. med., Frankfurt Hauner, Hans, Prof. Dr. med., München

Internisten und das (zentrale) Nervensystem Kraft, Johannes, Prof. Dr. med., Coburg Hofmann, Werner, PD Dr. med., Neumünster Multimorbidität und Polypharmazie Denkinger, Michael, Prof. Dr. med., Ulm Burkhardt, Heinrich, PD Dr. med., Mannheim

Prävention und Rehabilitation Halle, Martin, Prof. Dr. med., München Lüttje, Dieter, Prof. Dr. med., Osnabrück Versorgungsstrukturen - Versorgungsforschung Parhofer, Klaus, Prof. Dr. med., München Grandt, Daniel, Prof. Dr. med., Saarbrücken

"Young investigators - the future is now" Espinola-Klein, Christine, Prof. Dr. med., Mainz Malek, Nisar, Prof. Dr. med., Tübingen

\section{Programm - Kommission DGIM 2018}

Sieber, Cornel, Prof. Dr. med., Nürnberg/Regensburg (Vorsitzender)

Albus, Christian, Prof. Dr. med., Köln Bauersachs, Johann, Prof. Dr. med., Hannover Bischoff, Stephan C., Prof. Dr. med., Stuttgart Brunkhorst, Reinhard, Prof. Dr. med., Hannover Denkinger, Michael, Prof. Dr. med., Ulm Ertl, Georg, Prof. Dr. med., Würzburg Freiberger, Ellen, PD Dr. phil., Nürnberg Hach-Wunderle, Viola, Prof. Dr. med., Frankfurt Heppner, Hans Jürgen, Prof. Dr. med., Schwelm Hertenstein, Bernd, Prof. Dr. med., Bremen Hoffmann, Ute, Prof. Dr. med., Regensburg Hübner, Thilo, Mannheim Jung, Norma, PD Dr. med., Köln Kluge, Stefan, Prof. Dr. med., Hamburg Kochanek, Matthias, Dr. med., Köln Kuhlencordt, Peter, PD Dr. med., München Lammert, Frank, Prof. Dr. med., Homburg Lehnert, Hendrik, Prof. Dr. med., Lübeck Märker-Herrmann, Elisabeth, Prof. Dr. med., Wiesbaden Müller-Ladner, Ulf, Prof. Dr. med., Bad Nauheim Pfeifer, Michael, Prof. Dr. med., Donaustauf Püllen, Rupert, PD Dr. med., Frankfurt Schumm-Draeger, Petra-Maria, Prof. Dr. med., München Siegel, Erhard, PD Dr. med., Heidelberg Singler, Katrin, PD Dr. med., Nürnberg Stallmach, Andreas, Prof. Dr. med., Jena Strassburg, Christian, Prof. Dr. med., Hannover Trümper, Lorenz, Prof. Dr. med., Göttingen Vogelmeier, Claus, Prof. Dr. med., Marburg Virchow, J.-Christian, Prof. Dr. med., Rostock Volkert, Dorothee, Prof. Dr. rer.nat., Nürnberg Weber, Manfred, Prof. Dr. med., Köln 
Internist 2018 5 59 (Suppl 1): S1-S82

DOI 10.1007/s00108-018-0419-y

Online publiziert: 23. März 2018

๑) Springer Medizin Verlag GmbH, ein Teil von Springer Nature 2018

\section{Young Investigator Award}

\section{YIA 01 \\ Auswirkungen des Makrophagen-Phänotyps auf die Progression der Hyperurikämie-assoziierten CKD.}

Markus Sellmayr; Qiuyue Ma; Moritz Roman Hernandez Petzsche; Viviane Gnemmi; Hans-Joachim Anders; Stefanie Steiger

\section{Klinikum der Universität München, Med. IV, München}

Hintergrund: Es ist seit langem bekannt, dass eine enge Korrelation zwischen der Hyperurikämie und dem chronischen Nierenversagen (CKD) besteht. Allerdings ist bis heute nicht eindeutig geklärt, welches die Ursache und welches die Konsequenz ist. Auch die Bildung der Granulome in den Nieren der Patienten ist noch nicht im Detail verstanden, ebenso wenig wie deren Auswirkung auf den Krankheitsverlauf. Wir vermuten, dass durch das Forcieren einer anti-inflammatorischen M2-Makrophagen-Polarisation mit Adenosin die Progression der CKD verlangsamt werden kann.

Methodik: Sechs Wochen alte experimentelle Alb-creERT2; Glut9lox/lox (ki/ki) Mäuse und Alb-creERT2; Glut9+/+ (+/+) Kontrollmäuse wurden für eine Woche jeden zweiten Tag mit 1omg/kg KG Tamoxifen i.p. injiziert. Anschließend wurde den Mäusen ein fettreiches Inosin-haltiges Futter verabreicht. Ein Teil der ki/ki Mäuse wurde ab der zweiten Woche zusätzlich mit Adenosin (1mg/kg KG) behandelt. Harnstoff-Stickstoff (BUN), Kreatinin- und Harnsäurespiegel wurden im Serum gemessen, sowie die GFR bestimmt. Die mRNA-Expressionsmuster pro-fibrotischer bzw. pro-inflammatorischer Gene wurden mittels RT-qPCR nachgewiesen. (Immun-)Histologische Färbungen an Nierenschnitten bzgl. Entzündung und Fibrose wurden durchgeführt. Die Quantifizierung von Immunzellen erfolgte mittels Durchflusszytometrie.

Ergebnisse: Die ki/ki Tiere entwickelten eine signifikante Hyperurikämie $(18,84 \mathrm{mg} / \mathrm{dl}$ vs $3,23 \mathrm{mg} / \mathrm{dl} ; \mathrm{p}<0,01)$ im Vergleich zu den +/+ Mäusen. Auch der BUN-Wert $(125.76 \mathrm{mg} / \mathrm{dl}$ vs $52.27 \mathrm{mg} / \mathrm{dl} ; \mathrm{p}<$ $0,05)$, sowie der Serum-Kreatinin-Spiegel $(1,95 \mathrm{mg} / \mathrm{dl}$ vs $0,99 \mathrm{mg} / \mathrm{dl}$; $\mathrm{p}<0,001$ ) waren in der ki/ki Gruppe deutlich erhöht. Dagegen wiesen die ki/ki Mäuse eine signifikant niedrigere GFR auf $(72,17 \mu \mathrm{l} / \mathrm{min} /$ KG vs 336,48 $\mu \mathrm{l} / \mathrm{min} / \mathrm{KG} ; \mathrm{p}<0,001$ ). In den ki/ki Mäusen bildeten sich renale Harnsäurekristall-induzierte Granulome. F4/80+-Färbung sowie Durchflusszytometrie zeigten eine massive renale Makrophagen-Infiltration, wohingegen beides in den +/+ Mäusen ausblieb. RT-qPCR ergab eine Hochregulierung des Nierenschädigungsmarkers KIM-1 und der pro-inflammatorischen Zytokine IL- 6 und TNF- $a$ in den ki/ki Mäusen. Eine vermehrte renale Fibrose in den ki/ki Tieren zeigte sich histologisch und auf Transkriptionsebene. Interessant ist allerdings, dass nach Adenosin-Behandlung weniger Entzündung und Fibrose in den ki/ki Mäusen zu beobachten war, einhergehend mit einer verbesserten Nierenfunktion. Dies war mit einer erhöhten Anzahl an renalen anti-inflammatorischen $\mathrm{M}_{2}$-Makrophagen verbunden, bei einer gleichzeitig reduzierten Zahl pro-inflammatorischer Makrophagen.

Diskussion: In einem innovativen Tiermodell konnten wir zeigen, dass die Hyperurikämie mit begleitender Harnsäurekristall-assoziierter Granulom-Bildung zur CKD Progression beiträgt. Unter Gabe von Adenosin erhöhte sich die Anzahl der anti-inflammatorischen M2-polarisierten Makrophagen, wodurch eine Verlangsamung der Progression der CKD erreicht wurde.

\section{YIA 02}

Validität des SARC-F als Screening-Instrument zur Erfassung der Sarkopenie bei älteren Menschen mit funktionellen Einschränkungen

Tina Auerswald'; Anna Franke'; Susann Gotthardt'; Anne Hofmann'; Carolin Kraska'; Marina Mellou'; Ellen Freiberger ; Cornel C. Sieber ${ }^{1}{ }^{2}$; Daniel Schöne ${ }^{1}$

1 Institut für Biomedizin des Alterns, Friedrich-Alexander-Universität Erlangen-Nürnberg, Nürnberg; ${ }^{2}$ Krankenhaus Barmherzige Brüder Regensburg

Einleitung: Der alternsbedingte Verlust von Muskelmasse („Sarkopenie") gilt als eine wichtige Ursache des Gebrechlichkeitssyndroms und geht mit erhöhten Risiken für Funktionsverlust, Stürzen, Frakturen und Mortalität einher. Aufgrund der Relevanz dieses geriatrischen Syndroms existiert seit Oktober 2016 ein eigener ICD-10 Code (M62.84). Eine standardisierte Diagnostik und Therapie gibt es hingegen nicht. Der Bedarf an validierten, kostengünstigen und kurzen Screening-Instrumenten ist hoch. Als solches ist der SARC-F, ein Kurzfragebogen zur Identifizierung von Personen mit Sarkopenie entwickelt worden. Ziel dieser Studie war es, dessen Validität in einer Population funktionell eingeschränkter Senioren zu untersuchen.

Methodik: Selbständig lebende Personen wurden im Rahmen des Screenings einer randomisierten Kontrollstudie (SPRINTT-Studie, www.mysprintt.eu) untersucht und $\mathrm{N}=232$ in diese Sub-Studie eingeschlossen. Teilnehmer waren i) über 70 Jahre ( $\varnothing 80,0 \pm 5,6 ; 70-92 ; 71 \%$ weiblich), hatten ii) funktionelle Einschränkungen (Short Physical Per- 
formance Battery (SPPB) 3-9 (12=beste Funktionalität); $\varnothing_{7,0} \pm 1,6$ ) und iii) keine starken kognitiven Einschränkungen (MMST>23). Der SARC-F hat 5 Items (pro Item o-2 Punkte; o= keine Probleme); es werden subjektiv wahrgenommene Schwierigkeiten bei funktionell-körperlichen Alltagsaktivitäten und die Anzahl der Stürze im vergangenen Jahr erfasst. Der in der Literatur vorgeschlagene Schwellenwert für eine Sarkopenie liegt bei 4 Punkten und wurde auch hier appliziert. Der diagnostische Algorithmus der "European Working Group on Sarcopenia in Older People" (EWGSOP) wurde zur Bestimmung einer Sarkopenie angewandt (appendikuläre Muskelmasse - Dual-Röntgen-Absorptiometrie

\section{YIA 03}

Antikoagulative Erhaltungstherapie bei Tumorpatienten mit venöser Thromboembolie - Abbildung der Versorgungslandschaft mithilfe einer GKV-Routinedatenanalyse [CERTIFICAT]

\author{
Hanno Riess'; Albrecht Kretzschmar²; Melanie May ${ }^{3}$; Sebastian Schellong ${ }^{4}$; \\ Andreas Heinken ${ }^{5}$ \\ ${ }^{1}$ Charité Universitätsmedizin Berlin, Berlin; ${ }^{2}$ Städtisches Klinikum St.Georg- \\ Leipzig, Leipzig; ${ }^{3} \mathrm{HGC}$ GesundheitsConsult $\mathrm{GmbH}$, Düsseldorf; \\ ${ }^{4}$ StädtischesKlinikum Dresden, Dresden; ${ }^{5}$ Aspen Germany $\mathrm{GmbH}$, \\ München
}

Einleitung: Venöse Thromboembolien (VTE) treten häufig bei Tumorpatienten auf und gehören damit zu einer der häufigsten Komplikationen mit Auswirkungen auf die Gesamtprognose. Die entsprechende S2-Leitlinie empfiehlt eine Fortführung der initialen Antikoagulation mit niedermolekularen Heparinen (NMH). Es existieren bislang keine verlässlichen epidemiologischen Daten über die Versorgungsrealität in Deutschland. Die Nutzung von GKV-Routinedaten gewinnt in der Versorgungsforschung zunehmend an Bedeutung. Ziel der CERTIFICAT-Studie war es, die Versorgung von Tumorpatienten mit VTE mithilfe von GKV-Routinedaten zu beschreiben. Dabei sollte im ersten Schritt geprüft werden, wie und ob eine aussagekräftige Population identifiziert werden kann.

Methodik: Datenbasis bilden alters- und geschlechtsadjustierte GKV-Routinedaten (2011-2016) mit 4,1 Mio. Versicherten, das einer 5,5 \%igen Stichprobe der deutschen Bevölkerung entspricht. Es wurden zwei Patientenpopulationen aufgegriffen und in die Gruppen mit normalem oder hohem VTE-Risiko eingeteilt. Patienten mit normalem Risiko definieren sich durch 5 Kriterien: (1) Ambulant gesicherte $(\mathrm{M} 2 \mathrm{Q})$ oder stationäre Haupt- oder Nebendiagnose (HD/ND) "Krebs" (ICD 10 GM "C") im Zeitraum 2012- 2015, (2) erstmaliges Auftreten einer VTE ICD I80 als gesicherte ambulante oder stationäre HD/ND, (3) mind. ein Jahr vorher keine VTE, (4) Antikoagulation mit NMH (ATC $B 01 A B)$, VKA (Bo1AA) oder DOAK (B01AF) im selben Quartal der VTE (5) kein "hohes Ereignisrisiko". Die Einschlusskriterien für hohes Ereignisrisiko unterschieden sich von der ersten Gruppe durch das erstmalige Auftreten einer Krebsdiagnose.

Ergebnisse: Es konnten insgesamt 322.600 Tumorpatienten identifiziert werden. In der Normalrisikogruppe konnten 13.131 Patienten mit Tumordiagnose und initialer VTE im gleichen Quartal aufgegriffen werden. Mit Antikoagulation im gleichen Quartal bleiben 6.991 Versicherte. Getrennt von der Hochrisikogruppe waren 4.445 Patienten beobachtbar. Das Kriterium durchgehend versichert erfüllten 4.421. In der Hochrisikogruppe konnten 5.649 initiale Tumorpatienten mit initialer VTE im vorherigen, gleichen oder nachfolgenden Quartal gefunden werden. Mit Antikoagulation im vorherigen, gleichen oder nachfolgenden Quartal noch 2.901. Mit durchgehender Versichertenzeit blieb die Populationsgröße bei 2.892.
Zusammenfassung: Für die Analysen der Versorgungsforschungsstudie diente eine Gesamtpopulation von 7.313 Tumorpatienten, davon über die Hälfte aus der Normalrisikogruppe. Trotz Limitationen bei Studien mit Sekundärdaten, die den originären Zweck verfolgen, medizinische Leistungen abzurechnen, besitzt der Datensatz aufgrund seiner Stichprobengröße eine hohe Aussagekraft. Dadurch wird eine Grundlage geschaffen, die Versorgungsrealität von Tumorpatienten mit VTE darzustellen. Neben epidemiologischen Erkenntnissen für das deutsche Gesundheitswesen konnten so auch Versorgungsdefizite aufgedeckt werden, die in folgenden Publikationen näher beleuchtet werden.

\section{YIA 04}

Wie valide sind Aktivitätsdaten von mobilen Apple-Devices? Entwicklung einer telemedizinischen Patient Monitoring App zur Betreuung von Patienten nach Herzinsuffizienz-Hospitalisierung.

Stefanie Werhahn'; Henning Dathe'; Thorsten Rottmannn'; Thomas Franke ${ }^{2}$; Cath Wheeler ${ }^{3}$; Milano Fili ${ }^{3}$; Gerd Hasenfuß ${ }^{1}$; Tim Seidler ${ }^{4}$

${ }^{1}$ Universitätsmedizin Göttingen, Göttingen; ${ }^{2}$ Institut für Medizinische Informatik, Göttingen; ${ }^{3}$ Medopad Ltd, London U.K., London; ${ }^{4}$ Georg-August Universität Göttingen, Göttingen

Hintergrund: „Mobile Health“- („mHealth“-) Anwendungen eröffnen durch den Einsatz mobiler Endgeräte und entsprechender Gesundheitsapplikationen (Apps) neue Möglichkeiten der biomedizinischen Datenerfassung. Die Qualität der erhobenen Daten und der klinische Nutzen solcher mHealth-Applikationen sind aber noch unzureichend validiert.

Material und Methoden: In Kooperation mit Medopad Ltd (London, U.K.) entwickelten wir ein mHealth-Konzept zur telemedizinischen Betreuung von Patienten nach Herzinsuffizienz-Hospitalisierung mittels Apple iPhone und Watch. In einer Pilotstudie wurden zehn Patienten mit erstdiagnostizierter Herzinsuffizienz (LVEF $\leq 40 \%$, NYHA $\geq$ II) eingeschlossen und prospektiv über zwei Monate untersucht. Die Probanden erhielten je ein iPhone 6SE und eine Apple Watch der ersten Generation als Leihgeräte und wurden zur täglichen Eingabe von Daten (Blutdruck, Körpergewicht, Bestätigung der Medikamenteneinnahme, etc.) in die medopd-App aufgefordert. Über das Health \& Activity Framework konnte die medopad-App zusätzlich auf Gesundheitsdaten von iPhone und Watch (Aktivitätsdaten, Herzfrequenzprofil) zugreifen. Die gesammelten biomedizinischen Daten wurden kontinuierlich über eine verschlüsselte Verbindung an den Prüfarzt transferiert. Zusätzlich zur telemedizinischen Betreuung und Erfassung der Patientenzufriedenheit, wurden die o.g. mHealth-Daten etablierten Mess- und Untersuchungsmethoden (Echokardiographie, NTproBNP, Spiroergometrie, Langzeit-EKG und Herzinsuffizienz-Fragebögen etc.) gegenübergestellt, die bei Studieneinschluss sowie nach 1 und 2 Monaten durchgeführt wurden. Ein neu entwickelter Device-basierter 6 MWT wurde mit dem standardisierten 6 MWT verglichen.

Ergebnisse: Rating-Fragebögen erbrachten hohe Werte für die Benutzerfreundlichkeit, Funktionalität der App und Patientenakzeptanz. Über die zwei Monate zeigte sich eine signifikante Zunahme des Wochenmittels der täglichen Schrittzahl (2880 Schritte/Tag \pm 2318 bei Studieneinschluss und 6095 Schritte/Tag \pm 4158 bei Studienende; $p<$ 0.0001), die mit einer Verbesserung der konventionellen Parameter (LVEF im TTE, Rückgang von NT-proBNP, Spiroergometrie) korrelierte. Der Vergleich der Gehstrecke des konventionellen 6MWT mit dem Device-basierten 6 MWT war mit $539 \mathrm{~m} \pm 158$ vs. $506 \mathrm{~m} \pm 87,2$ nicht signifikant unterschiedlich und ergab eine statistisch signifikante Korrelation $(\mathrm{r}=0,879$; $\mathrm{P}$ 


\section{YIA 05}

Polypharmacy increases the risk of adverse events in patients receiving oral anticoagulation therapy - Results from the thrombEVAL study

Lisa Eggebrecht'; Markus Nagler'; Sebastian Göbel'; Heidrun Lamparter $^{1}$; Karsten Keller'; Christoph Bickel ${ }^{2}$; Michael Lauterbach ${ }^{3}$; Christine Espinola-Klein'; Roland Hardt'; Thomas Münzel'; Philipp Sebastian Wild'; Jürgen Prochaska'

${ }^{1}$ Universitätsmedizin Mainz, Mainz; ${ }^{2}$ Bundeswehrkrankenhaus Koblenz,Mainz; ${ }^{3}$ Krankenhaus der Barmherzigen Brüder Trier, Trier

Background: Polypharmacy is perceived as an important challenge in daily clinical practice, since it has been demonstrated to be related with adverse outcome. Although polypharmacy has been reported to potentially increase the frequency of negative events in several conditions, evidence on the relevance of polypharmacy for the clinical outcome of anticoagulated patients is currently limited.

Methods: Information on 2,011 individuals receiving vitamin K-antagonists was available for analysis from the prospective multi-centre thrombEVAL study (NCT01809015). Data were obtained from clinical visits, computer-assisted interviews, and laboratory measurements. Information on anticoagulation-specific and non-specific outcome was obtained during a three year follow-up period and subsequently validated via medical records. Information on medication was classified according to the ATC code list. Polypharmacy was defined as the intake of $\geq 5$ drugs. Cox proportional hazard regression models were applied to assess the relevance of polypharmacy for specific outcomes under consideration of potential confounders.

Results: In the study sample, the prevalence of polypharmacy was $84.1 \%(n=1,691)$. In detail, $44.7 \%(n=899)$ of subjects received $5-8$ drugs and $39.4 \%(n=792) \geq 9$ drugs. The mean number of medications used at baseline was $7.8 \pm 3.3$. During the three year follow-up period, the cumulative incidence of clinically-relevant bleeding increased across groups in a dose-dependent relationship: 5.0 [3.3/7.1] events per 100 patient-years (py) for individuals without polypharmacy, 8.5 [7.2/10.1] events per 10opy for individuals with 5-8 drugs, and 12.8 [11.0/14.9] per 100py for individuals with $\geq 9$ drugs $(P<0.0001)$. Similarly, an increased risk for hospitalization and all-cause mortality, but not for thromboembolic events, was detected. Quality of anticoagulation therapy assessed by time in therapeutic range (TTR) was lower in individuals on $5-8$ drugs and $\geq 9$ drugs $(70.7 \%[53.4 \% / 85.2 \%]$ and $64.7 \%$ [46.4\%/81.9\%], respectively) compared to subjects without polypharmacy (73.4\% [49.9\%/90.2\%]). In addition, a significantly higher variability of INR measurements was found in presence of polypharmacy. In Cox regression analysis with adjustment for potential confounders (i.e. age, sex, comorbidities, cardiovascular risk factors and TTR), polypharmacy indicated an elevated risk for hospitalization (hazard ratio (HR) 1.44 [1.15/1.79], $\mathrm{P}=0.0012)$, and all-cause mortality (HR 1.76 [1.18/2.62], $\mathrm{P}=0.0052$ ) and bleeding (HR 1.48 [0.98/2.24], $\mathrm{P}=0.065)$. The highest risk of adverse events was found for individuals receiving $\geq 9$ drugs (HRbleeding 1.62 [1.04/2.52], $\mathrm{P}=0.033$ ). Per additional drug, the risk of bleeding was increased by $7 \%(P=0.012)$.

Conclusion: Polypharmacy influences the quality of anticoagulation therapy and translates into an elevated risk of adverse events in anticoagulated patients, which may have implications for risk stratification and the management of anticoagulation therapy.

\section{YIA 06}

"Therapeutic Drug Monitoring“ (TDM) auf Standard-Antiinfektiva (TDMSAI) und "Drug Interaction Stewardship" (DIS) in der chirurgisch-operativ geprägten Intensivmedizin eines tertiären Zentrums

Florian Neuling; Uwe Lodes; Uwe Tröger; David Jacob; Frank Meyer

Universitätsklinikum Magdeburg A.ö.R., Magdeburg

Einleitung: Gestörte Organfunktionen und Organversagen, pathophysiologische Veränderungen beim schwer Erkrankten und in der Sepsis, laufende Organersatz-, Nierenersatz- und Leberersatzverfahren, vielfältig unterschiedliche Pharmakokinetik/Pharmakodynamik (PK/PD) von medikamentösen Substanzen sowie zahlreiche Medikamenteninteraktionen machen die Medikation des chirurgischen Intensivpatienten zunehmend schwer kalkulierbar. Ziel: Darstellung eines interdisziplinären Vorgehens im klinischen Alltag zur Optimierung der Vielfachmedikation als auch laufenden medikamentösen Therapie von Patienten vor indizierten Operationen oder Interventionen sowie im Rahmen des peri- und postoperativen intensivmedizinischen Managements.

Methode: Etablierung eines „Drug Interaction Stewardships" (DIS), analog und zeitgleich zum bereits etablierten Antibiotic Stewardship (ABS) in der Routine einer chirurgischen Intensivstation, sowie Erweiterung des Standard-TDM auf Standard-Antiinfektiva (Meronem, Piperacillin-Tazobactam, Ceftazidim, Linezolid, Vorikonazol, Fluconazol, Caspofungin), für die bisher kein TDM etabliert ist (TDMSAI) an einer konsekutiven Patientenkohorte über einen definierten Zeitraum im Rahmen einer prospektiven klinisch-systematischen "Single-center"-Beobachtungsstudie (tertiäres Zentrum) zur Reflexion des chirurgisch-intensivmedizinischen klinischen Alltags und als Beitrag zur chirurgischen Qualitätssicherung.

Ergebnisse: Im Zeitraum 01-2012 bis 08-2016 führten $1.454 \mathrm{kli}-$ nisch-pharmakologische Patientenvisiten zu 385 (26,5\%) Änderungen einer vorher vom erfahrenen Intensivmediziner eingestellten medikamentösen Therapie, am häufigsten in 156 (10,7\%) Fällen infolge einer neu kalkulierten PK/PD. 2.333 Proben TDMSAl ergaben in 1.130 Fällen $(48,4 \%)$ einen Talspiegel im gewünschten Bereich. In 427 (18,3\%) Fällen war wegen eines zu niedrigen und in 776 (33,3\%) Fällen wegen eines zu hohen Substanzspiegels eine Änderung der antiinfektiven Therapie nach Art, Dosis, Dosisintervall oder Appplikationsart erforderlich.

Schlussfolgerung: TDMSAI und DIS führen zu einer hohen Rate an Therapieänderungen einer bereits vorher von erfahrenen Intensivmedizinern eingestellten Therapie und können auf diese Weise die Qualität, insbesondere Wirksamkeit der medikamentösen Therapie bei chirurgischen Intensivpatienten entscheidend verbessern helfen 
YIA 07

\section{Die Rolle von Diabetes und HbA1c-Werten bei Patienten mit dekompensierter Leberzirrhose}

Tammo L. Tergast ${ }^{1}$, Hans Laser ${ }^{2}$, Svetlana Gerbel ${ }^{2}$, Michael. P. Manns ${ }^{1},{ }^{3}, 4$ Markus Cornberg ${ }^{1},{ }^{3,4}$, Benjamin Maasoumy ${ }^{1},{ }^{3}$,

${ }^{1}$ Klinik für Gastroenterologie, Hepatologie und Endokrinologie, Medizinische Hochschule Hannover, Carl-Neuberg-Str. 1, Hannover; ${ }^{2}$ Zentrum für Informationsmanagement (ZIMt), Medizinische Hochschule Hannover, Carl-Neuberg-Str. 1, Hannover; ${ }^{3}$ Deutsches Zentrum für Infektionsforschung DZIF, Partnersite Hannover-Braunschweig ${ }^{4}$ Zentrum für individualisierte Medizin (CIIM), c/o CRC Hannover, Feodor-Lynen-Str. 15, Hannover

Hintergrund und Ziele: Diabetes Mellitus Typ 2 (DM) ist eine häufige Komorbidität bei Patienten mit Leberzirrhose und ein bekannter Risikofaktor für Infektionen. Trotzdem sind Daten bezüglich des Einflusses von DM auf die Entwicklung einer spontan bakteriellen Peritonitis (SBP), einer schweren Komplikation bei Patienten mit dekompensierter Leberzirrhose und Aszites, selten. Glykosyliertes Hämoglobin A1c (HbA1c) ist der wichtigste Parameter für die 9o-Tages Überwachung von durchschnittlichen Blutzuckerwerten. Patienten mit Leberzirrhose haben eine erhöhte Hämolyse, welches die Kinetik des HbArc-Wertes beeinflusst, jedoch keine modifizierten Grenzwerte für den $\mathrm{HbA}$ c. In dieser Studie haben wir den Einfluss von $\mathrm{HbA} 1 \mathrm{c}$ auf die Inzidenz von SBP und die Letalität bei Patienten mit Leberzirrhose und Aszites untersucht.

Methoden: Insgesamt wurden 626 konsekutive Patienten, die zwischen Januar 2012 und Juni 2016 an der Medizinischen Hochschule Hannover ihre Parazentese erhielten, eingeschlossen und weiterverfolgt (mittlere Nachbeobachtungszeit: 200Tage). Die Klassifizierung als DM Patient erfolgte basierend auf den medizinischen Unterlagen der Patienten. Der HbA1c-Wert wurde zur Zeit der ersten Parazentese bestimmt. Zur Überlebensanalyse sind Log-rank Tests durchgeführt worden. Potentielle Risikofaktoren für SBP und Tod wurden in einem uni- bzw. multivariatem Modell überprüft. Zuletzt wurden zur Bestimmung von optimalen Cut-Offs ROC Analysen angerfertigt.

Ergebnisse: In der Gesamtkohorte befanden sich 152 Patienten mit DM (24\%). Während der Nachbeobachtungszeit entwickelten Patienten mit DM häufiger eine SBP (DM: $42 \%$ vs. kein DM: $33 \%$; HR = 1.5, $\mathrm{p}=0.03$ ). Besonders Patienten mit einem hohen $\mathrm{HbA} 1 \mathrm{c}$ hatten ein hohes Risiko für eine SBP: Als optimaler Cut-Off wurde in dieser Kohorte ein $\mathrm{HbA} 1 c$ von $6.4 \%$ identifiziert. Patienten mit einem $\mathrm{HbA} 1 \mathrm{c}$ von $\geq$ $6.4 \%$ hatten ein signifikant erhöhtes Risiko für eine SBP Entwicklung innerhalb von 90 Tagen im Vergleich mit Patienten, die einen $\mathrm{HbA} 1 \mathrm{c}$ $<6.4 \%$, bzw. keinen DM hatten ( $\mathrm{HbA}_{1 \mathrm{c}} \geq 6.4 \%$ : $66 \%$ vs. $\mathrm{HbA} 1 \mathrm{c}<6.4 \%$ : $25 \% ; \mathrm{HR}=4.16 ; \mathrm{p}=0.0002$, bzw. $\mathrm{HbA} 1 \mathrm{C} \geq 6.4 \%$ : $66 \%$ vs. kein DM: $26 \%$; $\mathrm{HR}=6.3 ; \mathrm{p}$

\section{YIA 08}

Lamina propria of the small intestine provides survival niches for memory plasma cells

Dominik Lammerding'; Qingyu Cheng'; Magdalena Kraft'; Karolin Pollock²; Andreas Radbruch'; Falk Hiepe ${ }^{3}$; Anja Hauser ${ }^{2}$; Bimba Franziska Hoyer ${ }^{4}$

'Deutsches Rheuma-Forschungszentrum Berlin (DRFZ), Berlin; ${ }^{2}$ Deutsches Rheuma-Forschungszentrum Berlin (DRFZ) und Charité Universitätsmedizin Berlin, Berlin; ${ }^{4}$ Universitätsklinik Schleswig-Holstein (UKSH), Kiel

Background: Autoreactive long-lived plasma cells (PCs) have been shown to play a major role in the pathogenesis of systemic lupus erythematosus, the prototype of systemic autoimmunity. Lymphatic organs such as spleen and bone marrow as well as inflamed organs such as inflamed kidneys have been shown to harbor longlived memory plasma cells. These cells are refractory to therapies with cyclophosphamid but have been shown to be susceptible to proteasome inhibitors. In protective humoral memory, mucosal memory plasma cells have been shown to play an important role. In this project, the intestinal lamina propria in lupus prone NZB/W F1 mice was analyzed for the presence of longlived plasma cells.

Methods: NZB/W mice of different ages were EdU-fed for 14 days in order to quantify the frequency and number of long-lived plasma cells in the lamina propria of the small intestine. Cells were analyzed by flow cytometry for the presence of plasma cell markers (Kappa, $\mathrm{CD} 138$ ) and EdU incorporation. Elispot was used to detect the number of autoreactive plasma cells and the presence of autoreactive antibodies in cell culture supernatants was determined by ELISA.

Results: We could show here that about $80 \%$ of the small intestine PCs in aged (7 month old) NZB/W mice with symptomatic lupus nephritis are indeed long-lived memory plasma cells. Using Elispot and ELISA we could show, that within this population of intestinal plasma cells, dsDNA-specific plasma cells are included, in the majority of $\lg A$ isotype.

Conclusion: In conclusion we were able to demonstrate that the intestinal lamina propria is a major compartment for long-lived plasma cells in a mouse model for systemic lupus erythematosus. It still needs to be shown whether the autoreactive mucosal plasma cells are also in their majority longlived and in how far they contribute to systemic autoimmunity. Further analysis is also needed with regard to their isotype, the present cytokine milieu and the role of neighboring cells. Nevertheless, the presence of this important memory plasma cell compartment has to be taken into account in considering treatment strategies and efficiency.

\section{YIA 09}

Wirkung unterschiedlicher Januskinase-Inhibitoren auf synoviale Fibroblasten von Patienten mit Rheumatoider Arthritis

Magnus Diller'; Marie-Lisa Hülser'; Stefan Rehart ${ }^{2}$; Martin Fleck³ ${ }^{3}$ Elena Neumann'; Ulf Müller-Ladner ${ }^{1}$

${ }^{1}$ Campus Kerckhoff, Justus-Liebig-Universität Gießen, Bad Nauheim; ${ }^{2}$ Agaplesion Markus Krankenhaus, Frankfurt; ${ }^{3}$ Asklepios Klinikum Bad Abbach, Universitätsklinikum Regensburg, Regensburg

Einleitung: Die Januskinase-Inhibitoren (JAKi) Tofacitinib und Baricitinib sind seit ${ }^{2017}$ in der EU zur Behandlung der Rheumatoiden Arthritis (RA) zugelassen. Weitere JAKi wie Peficitinib, Filgotinib und Upadacitinib werden aktuell noch in klinischen Studien erprobt. Die Effekte der Substanzen werden maßgeblich durch die Hemmung des IL-6-Signalwegs in Immunzellen vermittelt. Die Wirkung der JAKi auf aktivierte synoviale Fibroblasten von Patienten mit Rheumatoider Arthritis (RASF) ist bisher aber nur teilweise bekannt.

Ziel: Diese Studie vergleicht den Effekt unterschiedlicher JAKi auf die pro-inflammatorische Antwort von RASF.

Methoden: Die RASF wurden aus Synovialgewebe von Patienten mit bekannter RA im Rahmen einer Gelenksersatzoperation isoliert. Die Zellen wurden anschließend mit unterschiedlichen Konzentrationen der JAKi bzw. der Lösungsmittelkontrolle und mit IL-1 $\beta$ (10 oder $20 \mathrm{ng} /$ $\mathrm{ml}$ ) oder Onkostatin M (OSM, $100 \mathrm{ng} / \mathrm{ml})$ stimuliert. Nach 17, 18 oder 24 $\mathrm{h}$ wurden die Überstände abgenommen und die Konzentration von IL-6 mittels ELISA gemessen. Es wurde außerdem ein Assay durchgeführt, der die gleichzeitige Messung der Viabilität, Zytotoxizität und 
Apoptoserate ermöglicht, um mögliche Zytotoxizitäts-Effekte der JAKi auszuschließen.

Ergebnisse: In einem Vorversuch wurden die RASF zunächst mit den entsprechenden JAKi ( $1 \mu \mathrm{M}$ und $10 \mu \mathrm{M})$ für $2 \mathrm{~h}$ vorbehandelt und dann mit IL1- $\beta$ (20 ng/ml) für $18 \mathrm{~h}$ stimuliert. Bei der höchsten Konzentration von $10 \mu \mathrm{M}$ senkten Peficitinib, Filgotinib und Upadacitinib die IL-6-Ausschüttung der RA-FLS, wohingegen Tofacitinib und Baricitinib keine Wirkung zeigten. Sowohl Tofacitinib als auch Baricitinib reduzierten die Zytokin-Ausschüttung nur, wenn spezifisch der IL-6-Signalweg mit OSM aktiviert wurde $(n=3)$. Um den klinisch relevanten Konzentrationsbereich von $0,01 \mu \mathrm{M}$ bis $5 \mu \mathrm{M}$ zu untersuchen, wurden erneut RASF mit Filgotinib und Peficitinib für $2 \mathrm{~h}$ vorbehandelt und dann mit IL1- $\beta$ (10 ng/ml) für $17 \mathrm{~h}$ stimuliert. Peficitinib senkte bei $5 \mu \mathrm{M}$ den IL-6-Spiegel um $66 \%$ verglichen mit der Kontrolle IL1- $\beta$ ( $p$ kleiner als $0,01, n=5$ ). Bei $1 \mu \mathrm{M}$ zeigte sich ein Trend zu einer Reduktion um $24 \%(p=0,12, n=5)$. Filgotinib verursachte bei diesen Konzentrationen keine Veränderungen der IL-6-Spiegel. Diese Effekte von Peficitinib lassen sich nicht durch Zytotoxizität bzw. gesteigerte Apoptose erklären $(n=2)$.

Schlussfolgerung: Peficitinib reduziert die durch IL-1 $\beta$ ausgelöste proinflammatorische Antwort der RASF und scheint daher Tofacitinib und Baricitinib bezüglich der Wirkung auf RASF überlegen zu sein.

\section{YIA 10 \\ Corticosteroids potently abrogate RIPK1-independent necro- sis induced by Mycobacterium tuberculosis - implications for host-directed therapies}

Jessica Gräb'; Edeltraud van Gumpel'; Sandra Winter'; Andrew Cato ${ }^{2}$; Christoph Hölscher ${ }^{3}$, Jan Rybniker ${ }^{1}$

${ }^{1}$ Uniklinik Köln, Köln; ${ }^{2}$ Karlsruhe Institute of Technology, Karlsruhe; ${ }^{3}$ Forschungszentrum Borstel, Borstel

Tuberculosis (TB) continues to be one of the most deadly infectious diseases in humans. In 2016, 1.7 million people died from TB. The emergence of multi-drug resistant (MDR) and extensively-drug resistant (XDR) strains remains a public health crisis and has intensified research efforts to develop new therapeutic approaches, like host directed therapies (HDT). Mycobacterium tuberculosis (Mtb) strongly influences gene expression and cell-signaling in infected cells, providing targets for host-modulating therapies to shorten treatment periods, to reduce tissue damage and to lower the risk of relapsing disease.

Corticosteroids as HDT in combination with antibiotic treatment have been shown to reduce mortality in all forms of TB. However, the underlying mechanism of action is poorly understood. We have generated data showing that corticosteroids are highly effective in preventing Mtb-induced cytotoxicity in several cell types, including primary human macrophages. An intriguing finding since corticosteroids are the only approved HDT for TB. We were now able to link the protective effect of corticosteroids to the activation of MAPK phosphatase-1 (MKP-1). MKP-1 is essential for protection from hyperinflammation as well as tissue damage in autoimmune diseases by inactivating the stress responsive mitogen-activated protein kinases (MAPK). We show that host cell infection with Mtb leads to phosphorylation of p38 MAPK. Pretreatment with dexamethasone abrogates Mtb-induced p38 MAPK phosphorylation. A cytoprotective effect was also seen after treatment with the p38 MAPK inhibitor doramapimod, a substance currently tested in clinical trials against autoimmune diseases. This clearly links p38 MAPK phosphorylation to the cytotoxic effect mycobacteria exert on their host cells. Despite the well-known involvement of MAPKs in apoptosis, we show that the pan caspase inhibitor Z-VAD-FMK is not able to reduce Mtb-dependent host cell death. Therefore, we explored the role of necrosis effector proteins downstream of p38 MAPK, such as the release of the chromatin protein high mobility group box 1 (HMGB1). This pro-inflammatory protein can be detected in large amounts in body fluids of TB-infected patients. We show that this increased release of HMGB1 can be significantly reduced by pretreatment with dexamethasone and doramapimod in vitro. To determine the type of necrosis induced by Mtb, infected cells were treated with selective inhibitors of necroptosis, necrostatin-1 and necrostatin-5. However, necrostatin had no protective effect, leading to the assumption that Mtb induces a receptor-interacting serine/threonine-protein kinase 1 (RIPK1)-independent form of necrosis. Linking corticosteroids and p38 MAPK to necrotic cell death is a novel and unexpected finding.

Our data suggest that $\mathrm{HMGB}_{1}$ can act as a quantitative marker for necrosis in TB and that P38 MAPK inhibitors are potential substitutes for corticosteroids providing a more specific and tailored HDT in TB.

\section{"Young investigators - the future is now" I}

\section{PS001}

Einflussparameter auf das Überleben nach Resektion von solitären Lebermetastasen kolorektaler Karzinome - mitteldeutsche bi-institutionelle Studie

Sara Acciuffi'; Frank Meyer'; Roland S. Croner'; Astrid Bauschke²; Utz Settmacher $^{2}$; Hans Lippert ${ }^{3}$; Stefanie Wolff'; Annelore Altendorf-Hofmann ${ }^{2}$

'Universitätsklinikum Magdeburg A.ö.R.; ${ }^{2}$ Universitätsklinikum Jena; ${ }^{3}$ Otto-von-Guericke-Universität Magdeburg

Einleitung: Anhand der Daten aus 2 mitteldeutschen chirurgischen Kliniken aus den letzten 20 Jahren stellten wir uns die Frage, welche Ergebnisse bei dieser Gruppe zu erreichen sind.

Material und Methoden: Wir untersuchten eine konsekutive Serie von 374 prospektiv dokumentierten Patienten, die zwischen 1994 und 2014 in wegen einer solitären Lebermetastase eines kolorektalen Karzinoms kurativ behandelt wurden und die Leberresektion mindestens 3 Monate überlebten. Berechnet wurde das beobachtete Überleben (Zielkriterium Tod jeder Ursache).

Ergebnisse: 207 Patienten wurden vor 2004 leberreseziert, 167 von 2004-2014. Das mediane Alter aller Patienten betrug 63 Jahre. Bei 220 Patienten (59\%) traten Metastasen synchron mit dem Primärtumor auf, 154 metachron im Verlauf, davon 100 (24\%) nach mehr als 2 Jahren. 218 Primärtumoren (58\%) waren im Kolon, 156 im Rektum lokalisiert. Sie zeigten zu 81\% eine pT-Kategorie 3/4, zu 60\% regional Lymphknotenmetastasen ( $37 \% \mathrm{pN1}, 23 \% \mathrm{pN}$ ) und zu $17 \%$ Malignitätsgrad 3. 108 Lebermetastasen (29\%) waren größer als $5 \mathrm{~cm}$. Bei den Merkmalen Alter, pT/pN-Kategorie des Primärtumors, Metastasen-Durchmesser und tumorfreies Intervall zwischen Primärtumorbehandlung und Metastasen-Diagnose fand sich kein statistisch signifikanter Unterschied in der Verteilung zwischen beiden Kliniken. Statistisch signifikant unterschiedlich waren die Häufigkeit lokaler Exzisionen ( $p_{5} \mathrm{~cm}(p=0,010)$ hatten. Alle anderen Merkmale hatten keinen statistisch signifikanten Einfluss auf das beobachtete Überleben.

Schlussfolgerung: Patientenalter, multiple Lymphknotenmetastasen beim Primärtumor und der Metastasen-Durchmesser zeigten univariat einen statistisch signifikanten Einfluss auf das Langzeitüberleben. 


\section{PS002}

\section{8,4 Promille in 30 Minuten - ein Fallbericht zu einer massiven Alkoholintoxikation}

Cagla Nur Demirci; Constantin Maier-Stocker; Adela Georgescu; Franziska Kocheise; Michael Selgrad; Martina Müller; Stephan Schmid

Uniklinikum Regensburg, Regensburg

Auch wenn mittlerweile bereits vor einem mäßigen Alkoholkonsum gewarnt wird, ist weiterhin eine hohe gesellschaftliche Akzeptanz für regelmäßigen, moderaten Alkoholkonsum zu verzeichnen. Das „binge drinking“ kann jedoch durch die in kurzer Zeit massiv zugeführte Alkoholmenge ein lebensbedrohliches Ausmaß erreichen. Denn das Stadium des Unwohlseins wird übersprungen und nur das Koma beendet das Trinken. Ab circa 3,\%o steigt statistisch die Letalität. Bei über ${ }^{5 \%} \%$ rechnet man mit einer Mortalität von ${ }^{50} \%$.

Wir berichten von einer 27-jährigen Patientin, die in einer Damentoilette eines Einkaufzentrums mit einem GCS von 3 neben zwei Flaschen Alkohol aufgefunden wurde. Sie wurde bereits vom Notarzt intubiert. Laborchemisch zeigte sich initial ein Blutalkoholspiegel von $8,3 \%$ o und eine metabolische Azidose $(\mathrm{pH}=7,22)$. Bei Anisokorie wurde ein CCT veranlasst. Diese und die Kontrolle im Verlauf waren unauffällig. Röntgenologische stellte sich eine Aspirationspneumonie dar. Bronchoskopisch konnte hier Alkohol (Wodka) mobilisiert werden. Es wurde eine empirische Antibiose mit Ampicillin/Sulbactam und Clarithromycin begonnen. Bei einer Hypothermie von $34,1^{\circ} \mathrm{C}$ wurde die Patientin aktiv erwärmt. Der initiale Serumalkoholspiegel von 8,35\%o bestätigte sich in der Kontrollmessung (7,25\%o), so dass noch am Aufnahmetag eine Hämodialyse durchgeführt wurde. Unter Dialyse fiel der Alkoholspiegel sukzessive. Circa18 h nach Hospitalisation entwickelte sich ein akutes Leberversagen mit einer Blutungsneigung aus allen liegenden Kathetern. Laborchemisch zeigte sich ein akutes $\mathrm{Ge}$ rinnungsversagen mit einem INR $>7,55$, Quick

\section{PS003 \\ Myocardial Fibrosis predicts 10-Year-Survival in Patients Under- going Aortic Valve Replacement}

Bastian Fries'; Sebastian Herrmann²; Tim Salinger ${ }^{2}$; Dan Liư ${ }^{2}$; Kai Hu²; Daniel Gensler ; Jörg Strotmann ${ }^{3}$; Martin Christa ${ }^{2}$; Meinrad Beer'; Stefan Gattenlöhner ${ }^{5}$; Stefan Störk ${ }^{6}$; Wolfram Völker ${ }^{2}$; Constanze Bening ${ }^{2}$; Kristina Lorenz ${ }^{7}$; Rainer Leyh ${ }^{2}$; Stefan Frantz ${ }^{2}$; Georg Ertl' ${ }^{2}$; Frank Weidemann ${ }^{5}$; Peter Nordbeck ${ }^{2}$

'Städtisches Klinikum Karlsruhe; ${ }^{2}$ Universitätsklinikum Würzburg; ${ }^{3}$ Städtisches Krankenhaus Kiel; ${ }^{4}$ Universitätsklinikum Ulm;

${ }^{5}$ Universitätklinikum Gießen-Marburg; ${ }^{6}$ Deutsches Zentrum für Herzinsuffizienz; ${ }^{7}$ Leibnitz Institute for Analytical Sciences; ${ }^{8}$ Katharinen Hospital Unna

Background: Long-term data on evolution and clinical impact of myocardial fibrosis in valvular heart disease is scarce.

Methods and Results: In this 10-years extension of a prospective study in patients undergoing conventional aortic valve replacement (AVR) due to symptomatic severe aortic valve stenosis (AS), the impact of myocardial replacement fibrosis (RF) on long-term outcome was assessed. Endomyocardial biopsies were acquired during AVR in 58 consecutive patients. RF was graded using the calculated percentage area of fibrosis and patients categorized as severe $(n=21)$, mild $(n=15)$, no fibrosis $(n=22)$. Echocardiography including strain imaging, as well as cardiovascular magnetic resonance (CMR) to assess late gadolinium enhancement were performed at baseline, one and ten years after AVR. Death of any cause occurred in 21 patients (38.9 \%): 3 (14.3\%) in the group without RF, 6 (42.9\%) in the mild RF group and $12(63.2 \%)$ in the severe RF group ( $p=0.006)$, resulting in the lowest cumulative survival for patients with severe RF (log rank $p=0.003)$. In the group without RF none died of cardiovascular cause. RF was found to be an independent predictor of survival (hazard ratio 1.271, $95 \%$ confidence interval: 1.032-1.564, $p=0.024$ ).

Conclusion: This 10-year follow-up study underlines the profound impact of replacement fibrosis with regard to cardiac and all-cause mortality in patients undergoing AVR for severe AS. Integrating CMR and echocardiographic functional imaging beyond ejection fraction quantification could help in clinical decision making to stratify patient prognosis with regard to myocardial longitudinal function and prevalence of replacement fibrosis.

\section{PS004}

Higher fluorodeoxyglucose uptake in brown adipose tissue measured by positron emission tomography is associated with underweight but not with weight loss in end-stage COPD

\section{Armin Frille'; Karen Geva Steinhoff'; Swen Hesse'; Osama Sabri'; Hartmut} Kuhn'; Hubert Wirtz ${ }^{2}$; Hans-Jürgen Seyfarth'

'Universitätsklinikum Leipzig; ${ }^{2}$ Universitätsklinikum Leipzig AöR

Introduction: Progressive chronic lung diseases often lead to pulmonary cachexia (PC). Here, we assume that the activity of brown adipose tissue (BAT) visualized by means of fluorodeoxyglucose (FDG) positron emission tomography (PET) interacts with PC-induced weight loss. We aimed to find out whether the BAT activity measured by PET reflects the metabolic state and the severity of chronic lung diseases.

Methods: One hundred and four patients with end-stage chronic obstructive (COPD) and interstitial lung disease (ILD) underwent complete respiratory workup and an FDG-PET/computed tomography (CT) to rule out malignancy within the lung transplant evaluation process. Putative supraclavicular BAT regions were identified with CT. Mean standardized uptake values (SUVmean) of predefined volumes of interest were measured. Periumbilical regions with white adipose tissue (WAT) were similarly analysed. These data were normalized to each patient's liver uptake as a reference region. SUV ratios (SUVR) were compared with results from epidemiological and metabolic characteristics (e.g. body mass index [BMI]) and pulmonary function tests (e.g. FEV 1). To characterize cachexia, each patient's course of body weight (i.e. BMI) was assessed before and after the PET exam for a period of 5 years, respectively. Group comparisons and Spearman's correlation coefficients $(r)$ were calculated.

Results: SUVR in supraclavicular regions were significant higher in woman than in men $(\mathrm{P}=0,002)$. COPD patients showed an inverse correlation between FDG uptake in putative BAT regions and BMI $(P=0.002 ; r=-0.38)$. Interestingly, leaner COPD patients (BMI

\section{PS005}

The BMI's influence onto "morbidity and mortality" and survival rates after rectal cancer resections

\section{Björn Gebauer'; Ralf Steinert ${ }^{2}$; Henry Ptok'; Ronny Otto ${ }^{3}$; Ingo Gastinger ${ }^{3}$;} Hans Lippert ${ }^{3}$; Frank Meyer ${ }^{1}$

'Universitätsklinikum Magdeburg A.ö.R.; ${ }^{2}$ St Joseph Hospital; ${ }^{3}$ Otto-von-Guericke-Universität Magdeburg

Introduction: Overweight and obesity are healthcare challenge with globally growing prevalence. Literature data suggests an impairment of early postoperative outcome after general surgery but also an 
"obesity paradox" concerning better survival of patients being overweight or obese. The aim of this analysis was to evaluate the possible impact of obesity in rectal cancer surgery onto early postoperative and long-term oncological outcome.

Methods: A prospective multicenter observational study was conducted from 2008 to 2011 observing 68 items concerning pre-, peri-, postoperative (postop.) aspects $\&$ histological findings. These were reported by the clinic where the patient underwent surgery. A consecutive follow-up was performed if the patient signed a consent form. We subdivided the patients into underweight, normal weight, overweight \& obese patients as stated in the WHO definition for the body-mass-index (BMI).

Results: In total, 9,920 (BMI) patients were enrolled of whom $2.1 \%$ were underweight, $38.0 \%$ had normal weight, $40.5 \%$ overweight and $19.4 \%$ were obese. Mean age was 68 (range, 21-99) years (yr). Underweight patients showed more advanced diseases whereas overweight \& obese patients had lower tumor stages (according to UICC classification) compared to normal weight ones $(P<0.001)$. ASA score was worse in underweight \& obese patients $(P<0.001)$. Postop. morbidity (in total, $38.0 \%$ ) was worse in underweight patients due to more general complications $(P=$ mo.013) whereas obesity impaired surgery-related morbidity $(\mathrm{P}<0.001)$. In-hospital mortality was $3.1 \%$ in total without significant influences $(P=0.176) .5$-yr overall survival was $59.0 \%$ in total. Underweight patients showed decreased rates whereas overweight \& obese patients showed better rates $(P<0.001$ each). Disease-free survival (in total, $58.1 \%$ after $5 \mathrm{yr}$ ) was improved by a $B M I \leq 25.0 \mathrm{~kg} / \mathrm{m} 2(P<0.05$ each). Underweight did not show an influence compared to normal weight $(P=0.168)$. No effect was observed onto local recurrence rate (overall, $5.2 \%$ after $5 \mathrm{yr}$ ). Multivariate analysis identified overweight [HR=0.84 (95-Cl: 0.74-0.95); $\mathrm{P}=0.006]$ \& obesity [HR $=0.78$ (95- $\mathrm{Cl}: 0.66-0.91) ; \mathrm{P}=0.002]$ as independent factors for a better overall survival. Discussion Obesity leads to a higher risk of surgery-related complications without impairing in-hospital mortality. Our long-term results support the "obesity paradox" in rectal cancer surgery concerning the survival rates. The higher rates of general postop. morbidity in underweight patients may be caused by worse physical status preoperatively. Nevertheless, this correlation should be considered in the pre- \& postop. management.

\section{PS006 \\ Targetverteilung von PDE-5-Hemmern und Endothelin-1- Rezep- torantangonisten - eine immunhistologische Untersuchungen an humanen in-vivo-gewonnenen Pulmonalgefäßen-}

\section{Stefan Ferdinand Hertling; Marietta Süßkind von Schwendi}

Uniklinikum Regensburg, Regensburg

Hintergrund: Die pulmonale-arterielle Hypertonie (PAH) ist durch eine Erhöhung des Gefäßwiderstandes im Lungenkreislauf gekennzeichnet. Bei der Pathogenese nehmen vasomodulierende Faktoren eine Schlüsselrolle ein.

Material und Methode: 50 Patienten wurden während einer Lobektomie Segmente von Pulmonalarterie (PA) und Pulmonalvene (PV) entnommen. Mittels Immunhistologie wurden Endothelinrezeptoren ETa/ETb, sowie der Phosphodiester-5(PDE5)-Rezeptor untersucht. Zwei Untersucher werteten die Gefäßschnitte unabhängig und verblindet mit einem dafür konzipierten Auswertungsscore aus.

Ergebnis: Beide Gefäßarten hatten ein ähnliches Anfärbeverhalten. ETa und ETb-Rezeptoren färbten sich positiv (++) in allen Wandschichten. In Anlehnung an die Veränderungen der Wandschichten (z.B. Intimafibrose) zeigten sich in diesen Bereichen auch Veränderun- gen in der Rezeptordichte sowie deren Anfärbbarkeit. Pathologisch unauffällige Gefäße präsentieren ein homogenes Verteilungsmuster, während dies in pathologisch veränderten Bereichen sich änderte. So verstärkte sich die Anfärbbarkeit zu stark positiv (+++) von Eta/ Etb-Rezeptoren bei Intimaveränderungen. Dies wurde in PA und PV gleichermaßen beobachtet. Eine leichte postitve (+) Färbung des PDE5- Rezeptors konnte in PA und PV in allen Wandschichten dargestellt werden. Er zeigte eine schwächere Verteilung in beiden Gefäßen als die Endothelin-Rezeptoren. Bei vermehrter Neovaskularisation im peripheren Gefäßschnitt der PA verstärkte sich das Auftreten des PDE5-Rezeptors Richtung Gefäßlumen (++).

Schlussfolgerung: Die untersuchten Rezeptoren treten in allen Gefäßschichten auf. Ein Vorkommen auf einen Wandabschnitt allein scheint nicht der Fall zu sein. Bei strukturellen Veränderungen der Gefäßwand erscheint die Rezeptorverteilung verändert. PA und PV zeigen ein ähnliches Anfärbeverhalten für ETa-, ETb- und PDE5-Rezeptoren, sodass vasomodulierende Substanzen zur Therapie der PAH sowohl durch die Wirkung an PA als auch an PV wirken können.

\section{PS007 \\ Sind Pulmonalvenen (PV) den Pulmonalarterien (PA) gleichzu- stellen? - eine Antwort aus immunchemischer Sicht-}

\section{Stefan Ferdinand Hertling; Marietta Süßkind von Schwendi}

Uniklinikum Regensburg, Regensburg

Hintergrund: Klinische und pharmakologische Studien zur pulmonal-arteriellen Hypertonie (PAH) sind vorhanden. Allen gemein ist die Beschreibung eines optimalen Behandlungsregimes. Pulmonalarterien nehmen eine zentrale Stellung ein.

Material und Methode: 50 Patienten wurde während einer elektiven Lobektomie Segmente von PA und PV entnommen und histologische Gefäßquerschnitte gefärbt (HE, Sirius, SMA). Dabei handelt es sich um klinisch unauffällige Gefäßabschnitte. Zwei Untersucher haben die Präparate unabhängig und verblindet ausgewertet.

Ergebnis: Die HE-Färbungen von PA und PV zeigten den typischen Aufbau eines Gefäßes mit Intima, Media und Adventitia. In der Tunica Media beider Gefäßarten befand sich der höchste Anteil an Kollagenfasern und glatten Muskelzellen. Hierbei zeigte sich, dass diese im Verhältnis zur Gefäßgröße annährend gleichwertig bei beiden Gefäßtypen ausgeprägt waren. In $28 \%$ der PV konnten glatte Muskelzellen als abgegrenzte Zellinseln (SMA-positiv) in derer Tunica Adventitia nachgewiesen werden. Diese Zellinseln waren größer, je stärker die zugehörige Media schien. Innerhalb der drei Gefäßschichten konnten in beiden Gefäßtypen Veränderungen beobachtet werden ( $42 \%$ Wandverdickungen und $20 \%$ artherosklerotische Ausprägungen).

Schlussfolgerung: Diese histologische Untersuchung zeigt eine Ähnlichkeit im Aufbau der PA und PV. Da bisher das Hauptaugenmerk auf der PA lag, sollte nunmehr auch der PV mehr Beachtung geschenkt werden. Die beschriebenen abgegrenzten Zellformationen können für eine kontraktile Einheit sprechen, sodass die Kontraktionsfähigkeit beider Gefäßarten ähnlich ausfallen könnte. Für künftige pharmakokinetische Untersuchungen (Organbadversuche) wäre es sinnvoll beide Gefäßtypen zu untersuchen. Weiterhin kann nicht ausgeschlossen werden, dass der Wandaufbau der Gefäße und die beschriebenen Auffälligkeiten einen Einfluss auf die Rezeptorverteilung haben und somit die Therapie und Wirkungsweise von Medikamenten beeinflussen können. 


\section{PS008}

Targeted Therapie der Pulmonalen Hypertonie - eine immunhistochemische Untersuchung an PAH-erkrankten Gefäßen

\author{
Stefan Ferdinand Hertling; Marietta Süßkind von Schwendi \\ Uniklinikum Regensburg, Regensburg
}

Hintergrund: Klinische und pharmakologische Studien zur pulmonalen arteriellen Hypertonie (PAH) stehen zur Verfügung. Die Beschreibung eines optimalen Behandlungsregimes ist allen gemein. Pulmonalarterien (PA) nehmen eine zentrale Stellung ein. Vasoaktive Faktoren spielen eine Schlüsselrolle bei der Pathogenese. Phosphodiesterase-5-Inhibitoren und Endothelin-Rezeptor-Antagonisten sind wichtige Substanzklassen der PAH-Behandlung und werden dafür in der täglichen klinischen Praxis eingesetzt.

Methoden: 8 Patienten mit PAH und 8 Patienten ohne PAH, die eine elektive Lobektomie oder eine Lungentransplantation erhielten, wurden Segmente von PA und Pulmonalvenen (PV) entnommen. Diese wurden mittels immunhistochemischen Methoden fixiert, um die Verteilung von Endothelin-Rezeptoren (ET-A und ET-B) und des Enzyms Phosphodiester-5 (PDE-5) innerhalb der verschiedenen Wandstrukturen dieser Gefäße zu analysieren. Zwei Prüfer werteten die Segmente unabhängig und verblindet mit einem eigens dafür konzipierten Bewertungsscore aus.

Ergebnisse: Die Gefäßwand von PA und PV zeigte eine ähnliche Morphometrie (Intima, Media und Adventitia). Jeder der untersuchten Target-Enzyme zeigte sich gleichmäßig in beiden Gefäßarten über jede Gefäßwandschicht verteilt. Die Dichte von Phosphodiester-5 (PDE-5) -Enzym und Endothelin-Rezeptoren in den PAH-Gefäßen war höher im Vergleich zu den klinisch unauffälligen PA und PV. Pathologische Veränderungen in der Wandschicht (z. B. beginnende Intimafibrose) veränderten die Dichte und die Verteilung der untersuchten Targetenzyme. Dieser Effekt zeigte sich bei PA und PV der PAH erkrankten Gefäßen verstärkt.

Schlussfolgerungen: Target-Enzyme für ET-A, ET-B und PDE-5 waren in jeder Schicht von PA und PV vorhanden. Beide Gefäßarten scheinen gleichermaßen betroffen. Bei strukturellen Veränderungen in der Gefäßwand erscheint die Rezeptorverteilung verändert. Diese fällt in PAH-Gefäßen signifikant höher aus. Die untersuchten Stoffklassen können sich daher positiv auf den Gefäßtonus der PAH auswirken. Es ist anzunehmen, dass atherosklerotische Gefäßveränderungen eine Einflussgröße auf die Targetenzymverteilung in beiden Gefäßtypen sein können. Weitere elektrophysiologische und immunchemische Untersuchungen sind ausstehend.

\section{PS009 \\ Pulmonale Hypertonie - eine ultrastrukturelle Untersuchung an humanen PAH-Gefäßen}

Stefan Ferdinand Hertling; Marietta Süßkind von Schwendi; Karla Lehle

Uniklinikum Regensburg, Regensburg

Hintergrund: Die Pulmonalarterien (PA) werden typischerweise zur pharmakokinetischen Analyse neuer Medikamente zur optimalen Behandlung der pulmonalen arteriellen Hypertonie (PAH) verwendet. Vasoaktive Faktoren spielen eine Schlüsselrolle bei der Pathogenese. Es gibt verschiedene morphologische Pathologien, die für die Klassifizierung von Gefäßveränderungen bei pulmonaler Hypertonie kennzeichnend sind. Diese (histologische) Studie vergleicht die Gefäßmorphometrie PAH erkrankter menschlicher Lungengefäße mit humanen nicht-PAH erkrankten Gefäßen.
Methoden: 8 Patienten mit PAH und 8 Patienten ohne PAH, die eine elektive Lobektomie oder eine Lungentransplantation bekamen, wurden Segmente von PA und Pulmonalvenen (PV) entnommen. Die Nicht-PAH-Gefäßsegmente waren klinisch unauffällig. Histochemische Methoden wurden verwendet, um die ultrastrukturelle Untersuchung der Gefäßmorphometrie unter Berücksichtigung der Gefäßwände zu analysieren. Zwei Prüfer bewerteten die Segmente unabhängig und verblindet mit einem dafür konzipierten Bewertungsscore.

Ergebnisse: Die Gefäßwand von PA und PV zeigte eine ähnliche Konstruktion (Intima, Media und Adventitia). Veränderungen in der Gefäßmorphometrie und auch atherosklerotische Veränderungen wurden dokumentiert. Gefäßwandverdickungen, Fibrose, Atherome und erhöhte periphere Neovaskularisation wurden gefunden. Dieser Effekt wurde für PA und PV beider Studiengruppen gezeigt. Die Änderungen wurden in PA und in der entsprechenden PV dokumentiert. Der Grad der Veränderungen der atherosklerotischen Gefäßwand war in PAH-Gefäßen stärker ausgeprägt.

Schlussfolgerungen: Die Gefäße beider Studiengruppen zeigten Ähnlichkeiten. Unauffällige Gefäße und PAH-Gefäße zeigen atherosklerotische Veränderungen. Diese pathologischen Veränderungen ist bisher nur in den Pulmonalgefäßen PAH-erkrankter Gefäße beschrieben. Darüber hinaus scheinen PA und PV von diesen Gefäßveränderungen gleichermaßen betroffen zu sein. Daher können vasoaktive Mittel zur Behandlung von PAH in PA und PV wirken. Beide Arten von Gefäßen könnten ein wertvolles Instrument zur Analyse vasoaktiver Mittel für die Behandlung von PAH sein.

\section{PS010 \\ PCSK9-inhibitor treatment of cardiovascular high risk patients in clinical practice - new data regarding safety and effectivity}

Tim Hollstein ${ }^{1}$; Thomas Grenkowitz² ; Hubert Scharnagl ${ }^{3}$; Winfried März'; Elisabeth Steinhagen-Thiessen ${ }^{2}$; Ursula Kassner ${ }^{2}$

${ }^{1}$ Charité Universitätsmedizin Berlin; ${ }^{2}$ Charité Universitätsmedizin der Freien und der Humboldt Universität; ${ }^{3}$ Medizinische Universität Graz;

${ }^{4}$ Universitätsklinikum Heidelberg

Introduction: Only recently, Proprotein convertase subtilisin/kexin type 9 antibodies (PCSK9-I) became available to treat therapy-refractory hypercholesterolemia. The aim of this study is to provide new safety and efficacy data of PCSKg-I collected in clinical practice, with particular regard to subgroups in patients with diabetes mellitus or high Lp(a).

Methods: 600 patients with high and very high cardiovascular risk according to the European Society of Cardiology (ESC) guidelines, who did not achieve LDL-C goals under maximal tolerable lipid-lowering therapy or were statin-intolerant were selected to therapy with either alirocumab (150 mg or $75 \mathrm{mg}$ every two weeks) or evolocumab (140 $\mathrm{mg}$ every two weeks). Clinically-indicated laboratory tests were performed before and after one month of PCSKg-I treatment initiation.

Results: PCSK9-I treatment reduced total and LDL-C by 38 and 52 percent, respectively. Triglycerides (TG) decreased by 26 percent, HDL-C increased by 14.5 percent. LDL-C reduction correlated with baseline $L D L$ and $L p(a)$ reduction in subjects with elevated $L p(a)$. In patients with diabetes mellitus, baseline HbArc correlated with the triglyceride reduction, especially in VLDL. Half of the patients developed side effects during therapy, where cold symptoms and fatigue were the most common. 
Conclusions: Treatment with PCSK9-I seems to be a safe and effective way to further reduce LDL-C levels in cardiovascular high risk patients. Patients with diabetes mellitus seem to take advantage of a reduced $\mathrm{Hb} 1 \mathrm{Ac}$ and a higher triglyceride reduction, due to reduced VLDL and reduced TGs in LDL.

\section{PS011}

Quantitative und qualitative Analyse zur Beurteilung einer Änderung des Essverhaltens und des Wirkmechanismus eines nicht-invasiven neuartigen Medizinproduktes zur Gewichtsreduktion bei Übergewicht und Adipositas

Pia Hüttemann ; Aleksandra Nowak'; Jessica Königsmann ; Peter von Seck ${ }^{1}$; André Schmidt-Lucke'; Caroline Schmidt-Lucke ${ }^{1}$

${ }^{1}$ MEDIACC GmbH, Berlin; ' MEDIACC GmbH, Berlin; ${ }^{1}$ MEDIACC GmbH, Berlin; ${ }^{2}$ Praxis für Innere Medizin, Wiesbaden

Hintergrund: In evidenzbasierten Therapieprogrammen zur Gewichtsreduktion ist die erneute Gewichtszunahme nach vorangegangener Gewichtsreduktion typisch. Das Ziel dieser Studie war es daher die Hypothese zu untersuchen, ob die regelmäßige Nutzung eines neuartigen intra-oralen Medizinproduktes (Risikoklasse I) während der Nahrungsaufnahme zu einer Änderung des Essverhaltens und damit zu einer langfristigen Gewichtsreduktion führt.

Methodik und Ergebnisse: In die prospektive, randomisierte kontrollierte, einfach-verblindete, multizentrische Studie wurden 19 adipöse Patienten eingeschlossen (Alter 45,1 \pm 17,2; BMI 31,12 $[26,9-40,7] \mathrm{kg} / \mathrm{m} 2$, davon 3 Männer). Alle Patienten hatten in der Vergangenheit mindestens 2 frustrane Abnehmversuche erlebt. Während der Studie sollten sie keine Diät halten, essen ad libitum, keine Kalorien zählen, sich Zeit zum Essen nehmen und die Mahlzeit erst dann beenden, wenn sie satt waren. Die Schiene wurde während der ersten 3 Monate zu jeder Mahlzeit getragen, woraus sich eine Gesamttragezeit von 80 Stunden ergab. Die Follow-Up Zeit betrug insgesamt 12 Monate. In den ersten 3 Monaten reduzierten die Probanden ihr Körpergewicht signifikant $(p<0,005)$.

Mithilfe evaluierter, standardisierter Fragebögen sowie eines zweitägigen Essversuchen wurde der Einfluss der Schiene auf spezifische Parameter des Essverhaltens quantifiziert. Die Patienten kauten mit Schiene 3-mal häufiger pro Bissen $(p<0,001)$, sie benötigten doppelt so viel Zeit bis zum Schlucken pro Bissen $(p<0,001)$ und $60 \%$ mehr Zeit zum Beenden der Mahlzeit $(p<0,001)$. Zudem aßen sie $25 \%$ kleinere Portionen $(p<0,012)$, bemerkten die verzehrte Speisemenge besser $(p<0,004)$ und gaben keinen Hunger an, wenn sie direkt nach dem Essen dazu befragt wurden - trotz zuvor kleinerer verzehrter Speisemenge. Auch nach 3 Stunden hatten sie tendenziell weniger Zwischenhunger, als ohne Schiene $(p=0,10)$. Die Probanden hatten nach dem Essen mit Schiene weniger Appetit auf Süßes $(p<0,048)$ und Fettes $(p<0,004)$ und bemerkten im Verlauf der Studie eine Änderung des Essverhaltens $(p<0,001)$.

Zusammenfassung: Diese Ergebnisse zeigen, dass erstmalig ein nicht-invasives Medizinprodukt durch eine Änderung des Essverhaltens eine signifikante Gewichtsreduktion bei adipösen Patienten bewirkt, insbesondere bei freier Wahl der Speiseart und -menge. Daher stellt die Entwicklung dieses neuartigen Produktes in Kombination mit dem bestehenden leitlinienbasierten Basisprogramm eine vielversprechende Option zur Gewichtsreduktion bei pathologischer Adipositas dar. Diese Ergebnisse bilden die Grundlage für eine experimentelle Untersuchung der Änderung des Essverhaltens zur Gewichtsreduktion bei adipösen Patienten.

\section{PS012}

Prävalenz und prognostische Bedeutung der neuen echocardiographischen Diagnosealgorithmen für HFpEF bei CKD Patienten

Hannah Kaiser; Gunnar Heine; Insa Emrich; Kathrin Untersteller; Danilo Fliser; Stephan Schirmer; Michael Böhm

Universitätsklinikum des Saarlandes, Homburg;

Hintergrund: Die Herzinsuffizienz (HI) mit erhaltener Ejektionsfraktion (HFpEF) ist von ähnlicher epidemiologischer und prognostischer Bedeutung wie eine systolische $\mathrm{HI}$ (HFrEF). Anders als die HFrEF, die echokardiographisch eindeutig durch Bestimmung der Ejektionsfraktion (EF) diagnostiziert werden kann, waren bisherige echokardiographische Diagnosealgorithmen für HFpEF, welche pathologische Basis der HFpEF ist, für den klinischen Alltag oft zu komplex. 2016 wurden von der American Society of Echocardiography (ASE) und der European Association of Cardiovascular Imanging (EACl) daher neue echokardiographische Kriterien zur Diagnose der diastolischen HI veröffentlicht.

Wir wollten überprüfen, ob diese Diagnosekriterien auch auf Menschen mit chronischer Nierenerkrankung (CKD) übertragen werden können, oder ob sie in eine Überdiagnostik der HFpEF münden. Eine solche Überdiagnostik würde in einer unzureichenden Diskrimination von CKD Patienten mit erhöhtem Risiko für kardiovaskuläre Ereignisse münden. Daher untersuchten wir Prävalenz und prognostische Bedeutung der HFpEF nach ASE / EACI Kriterien in einer größeren Kohorte von CKD Patienten.

Methodik: Die prospektive CARE FOR HOMe Studie rekrutiert CKD Patienten im Stadium G2-G4; 526 von 575 eingeschlossenen Patienten erhielten bei Studieneinschluss eine standardisierte Echokardiographie. Gemäß den ASE und EACI Empfehlungen wurde die mediale und septale Bewegung des Mitralklappenanulus (e'), der linksatriale Volumen Index (LAVI), die maximale Geschwindigkeit des transtrikuspidalen Insuffizienzjets (TR-jet) und die transmitrale Einstromgeschwindigkeit (E) gemessen und der mitrale $E / e^{\prime}$ Index berechnet. Sind >2 der Parameter pathologisch verändert, wird die Diagnose der diastolischen $\mathrm{HI}$ gestellt. Bei genau 2 zutreffenden Parametern ist die Diagnosestellung „unklar“. Von der Auswertung ausgeschlossen waren Patienten mit inkompletten echokardiographischen Daten und Patienten mit eingeschränkter systolischer Herzfunktion, so dass 401 von 526 Patienten ausgewertet wurden. Alle Patienten wurden mittels standardisierter Interviews jährlich nachbeobachtet; primärer Endpunkt unserer Auswertung war die stationäre Aufnahme aufgrund kardialer Dekompensation.

Ergebnisse: Von den 401 ausgewerteten Patientendaten konnte bei 8\% (31 Pat.) eine HFpEF gesichert und bei 66\% (265 Pat.) ausgeschlossen werden. Bei den verbleibenden 26\% (105 Pat.) blieb die Diagnose echokardiographisch "unklar". Die Patienten mit $>2$ pathologisch veränderten Parametern hatten in Kaplan-Meier Analysen deutlich häufiger kardiale Dekompensationen als Patienten mit "unklarer" Diagnose; Patienten mit $<2$ veränderten Parametern hatten die beste Prognose (log rank $p<0.001)$.

Schlussfolgerung: Entgegen unserer Erwartungen resultierten die neuen Diagnosealgorithmen nicht in einer Überdiagnose der HFpEF bei CKD Patienten; anhand der drei vorgeschlagenen Kategorien lassen sich die Patienten prognostisch differenzieren. 


\section{"Young investigators - the future is now" II}

\section{PS013}

\section{A skeletal muscle derived and secreted myokine attenuates left ventricular dysfunction}

Badder Kattih ; Malgorzata Szaroszyk ${ }^{2}$; Mortimer Korf-Klingebiel ${ }^{2}$; Andrea Grund $^{2}$; Natali Froese ${ }^{2}$; Michael Wagner ${ }^{3}$; Oliver J. Müller'; Thomas Thum²; Hugo Albert Katus ; Ali El-Armouche ${ }^{3}$; Kai Christoph Wollert ${ }^{2}$; Johann Bauersachs ${ }^{2}$; Jörg Heineke'

${ }^{1}$ Medizinische Fakultät Mannheim der Universität Heidelberg; ${ }^{2}$ Medizinische Hochschule Hannover; ${ }^{3}$ Universitätsklinikum Carl Gustav Carus TU Dresden; ${ }^{4}$ Universitätsklinikum Schleswig-Holstein Campus Kiel; ${ }^{5}$ Universitätsklinikum Heidelberg

Background: Pathological remodeling during heart failure is characterized by the activation of different neurohormonal systems. While chronic activation of the sympathetic nervous system is harmful, increased natriuretic peptide (NP) levels are in principal beneficial, although their effectiveness is strongly impaired in heart failure, in part due to their rapid degradation. Musclin, a novel skeletal muscle-derived, secreted myokine is a specific ligand of the NP clearance receptor (Npr3), thereby inhibiting the degradation of natriuretic peptides.

Methods: Transaortic constriction (TAC) was used to induce longterm pressure overload and transition to heart failure with muscle wasting in $\mathrm{C} 57 \mathrm{BL} / 6 \mathrm{~J}$ mice. RNAseq in wasting skeletal muscle 12 weeks after TAC revealed a 3.5-fold reduction in Musclin expression, which was confirmed by RT-qPCR analysis. While Musclin as potential cardioprotective myokine is downregulated in skeletal muscle during heart failure, we assessed the functional consequence of Musclin overexpression in murine skeletal muscle as therapeutic approach.

Results: AAV6-mediated skeletal muscle specific overexpression of Musclin ameliorated myocardial fibrotic remodeling (Sirius red staining) and pulmonary congestion (lung weight/body weight ratio) compared to AAV6-Control treated mice 12 weeks after TAC. These beneficial effects were accompanied by improved cardiac function (echocardiography) 6, 9 and 12 weeks after TAC in mice treated with AAV6-Musclin. Improved systolic contractility and diastolic relaxation was also demonstrated in the AAV6-Musclin group by intraventricular pressure/volume analysis. Moreover, we investigated the impact of Musclin overexpression on cardiomyocyte contractility in isolated cardiomyocytes. Indeed, adult murine cardiomyocytes isolated from mice 8 week after TAC and AAV6-Musclin administration (vs. TAC and AAV6-Control) exhibited improved contractility at baseline and during beta1-adrenergic receptor stimulation. While NP production (RT-qPCR and Western blot analysis) in murine hearts was not different in AAV6-Musclin vs. AAV6-Control treated mice subjected to TAC, ANP plasma levels tended to be higher in AAV6-Musclin treated mice, suggesting less degradation of NPs. Administration of a selective Npr3 antagonist (AP-811) via osmotic pumps for 4 weeks partially blunted the Musclin induced increase in cardiac function, indicating that Musclin mediates its effects at least in part through enhancement of natriuretic peptide (NP) signaling .

Conclusion: Preventing disease progression in chronic heart failure by inter-tissue communication via AVV6-mediated overexpression of a novel skeletal muscle-derived and secreted myokine could be a promising therapeutic strategy for the treatment of chronic heart failure. Our data show that Musclin potently attenuates LV dysfunction following pressure overload at least in part through enhancement of natriuretic peptide (NP) signaling.
PS014 Unterschätzt der MELD-Score die Mortalität von Patienten mit nosokomial erworbener spontan bakterieller Peritonitis?

Markus Kimmann; Tammo Tergast; Hans Laser; Michael Peter Manns; Markus Cornberg; Benjamin Maasoumy

Medizinische Hochschule Hannover, Hannover;

Hintergrund und Ziele: Patienten mit dekompensierter Leberzirrhose sind eine vulnerable Patientengruppe mit hoher Mortalität. Häufig ist eine Lebertransplantation (LTx) die einzige kurative Behandlungsoption. Für die Organallokation in Deutschland ist der MELD-Score maßgeblich, welcher die kurzfristige Prognose (9o Tage) und somit die Dringlichkeit einer Transplantation widerspiegeln soll. Eine der häufigsten Komplikationen bei dekompensierter Leberzirrhose ist die spontan bakterielle Peritonitis (SBP). Gegenwärtig existieren lediglich begrenzte Daten bezüglich des prognostischen Wertes des MELD-Scores bei Patienten mit SBP. Das Ziel dieser Studie war zu untersuchen, ob der MELD-Score bei Patienten mit SBP die Prognose adäquat widerspiegelt.

Methode: Insgesamt wurden 608 konsekutive Patienten mit Leberzirrhose und Aszites eingeschlossen. Die Patienten wurden in drei Gruppen eingeteilt: Patienten ohne SBP (no-SBP) $(n=334)$, Patienten mit ambulant erworbener SBP (caSBP) $(n=59)$ und Patienten mit nosokomial erworbener SBP ( $n S B P)(n=215)$. LTx-freies Überleben (innerhalb von 90 Tagen) wurde mit dem Log-Rank-Test, potentielle Risikofaktoren mittels uni- und multivariater Cox-Regression analysiert.

Ergebnisse: Das Überleben war bei Patienten mit nSBP signifikant geringer als bei no-SBP Patienten (nSBP 60,5\% vs. no-SBP 79,6\%; $p$

\section{PS015}

Therapieeffizienz bei idiopathischer retroperitonealer Fibrose in einer retrospektiven, monozentrischen Analyse

Caroline Kumpert'; Stefanie Hahne'; Marcus Makowski ; Birgit Rudolph ${ }^{3}$; Gerd-Rüdiger Burmester ${ }^{3}$; Falk Hiepe ${ }^{2}$; Bimba Franziska Hoyer ${ }^{4}$

${ }^{1}$ Deutsches Rheuma-Forschungszentrum Berlin (DRFZ), Berlin; ${ }^{2}$ Charité Universitätsmedizin Berlin, Berlin; ${ }^{3}$ Charité - Universitätsmedizin Berlin, Berlin; ${ }^{3}$ Charité - Universitätsmedizin Berlin, Berlin; ${ }^{2}$ Charité Universitätsmedizin Berlin, Berlin; ${ }^{4}$ Universitätsklinik Schleswig-Holstein (UKSH), Kiel

Einleitung: Der Morbus Ormond (RPF) ist eine seltenen Erkrankungen. Klinisches Haupt-Symptom ist ein retroperitoneale Fibrose die zu Harnstau durch die Kompression der Harnleiter führt. Es gibt keine zugelassenen Therapien. In unserem Zentrum wird eine Cyclophosphamid-Bolus-Therapie über mindestens 6 Monate durchgeführt. Man geht davon aus, dass ein großer Anteil der IRF-Patienten an einer Unterform der IgG4-assoziierten Erkrankung leidet. Zum Therapieansprechen existieren für alle Gruppen nur wenige Daten.

Methoden: In einer retrospektiven Analyse wurden die Krankenakten der Patienten mit RPF unter Cyclophosphamid-Bolustherapie, die sich in den letzten 15 Jahren an der Charité in Behandlung befanden auf das klinische, serologische und radiologische Ansprechen nach 6 Monaten Therapie untersucht. Bei Patienten mit Biopsie vor Therapie erfolgte durch Pathologen eine Nachbefundung bezüglich des IgG4-Status.

Ergebnisse: Von insgesamt 20 Patienten, die in den letzten 15 Jahren an der Charité unter der Diagnose eines RPF mit Cyclophosphamid behandelt wurden, waren 7 Patienten bioptisch IgG4-positiv, 4 negativ und 1 unklar (zu wenig Material). Von 8 Pati- 
enten gab es keine Biopsie. Eine positive Patientin wurde nachträglich beim Nachweis einer Peritoneal-Karzinose ausgeschlossen.

IgG4-negative Patienten hatten initial ein signifikant größeres Volumen der retroperitonealen Masse als positive und unklare Patienten. Unter Therapie kam es nur in der positive und unklaren Gruppe zu einer signifikanten Reduktion der retoperitonealen Masse ( $p=0,03$ und o,0039). Die Nierenfunktion (Kreatinin) war in allen 3 Gruppen initial eingeschränkt (Kreatinin positiv 1,164 $\pm 0,31 \mathrm{mg} / \mathrm{dl}$, neg 1,038 $\pm 0,27$, unklar 1,52 mg/dl $\pm 0,27$ ) und es kam in keiner Gruppe zu einer signifikanten Verbesserung im Laufe der 6 Monate. IgG4-Konzentrationen im Serum waren nur für einzelne Patienten zum Zeitpunkt der Diagnosestellung verfügbar. Eine Aussage über den Verlauf des Antikörperspiegels ist somit in dieser Studie nicht möglich.

Schlussfolgerung: Insgesamt zeigt sich in der Hälfte aller Patienten mit RPF eine signifikante Reduktion der retroperitonealen Masse ohne das sich dies innerhalb der ersten 6 Monate in einer Verbesserung der Nierenfunktion niederschlägt. Weitere, prospektive Studien und ein längerer Beobachtungszeitraum sind notwendig, um über den Einfluss des Faktors "IgG4" eine Aussage machen zu können.

\section{PS016}

Etablierung eines innovativen Modells zur Kultivierung humaner Tumorzellen

Claudia Kunst; Martina Müller

Universitätsklinikum Regensburg, Regensburg;

Einleitung: Eine der großen Herausforderungen der modernen Medizin ist die Therapie komplexer Erkrankungen. Krankheitsbilder wie Krebs weisen stark schwankende Charakteristika auf und sind patientenindividuell sehr unterschiedlich. Je nach Mikromilieu des Tumors sowie Immunstatus und Vorgeschichte des Patienten kann eine Tumorerkrankung in variabler Form auftreten. Bisher wird anhand der Klassifizierung mittels bestimmter Oberflächen und Biomarker die Therapiestrategie festgelegt. Wünschenswert wäre jedoch eine individualisierte Strategie, die unter Berücksichtigung des individuellen Immunstatus und der Mikroumgebung des zu behandelnden Tumors erfolgt. Allerdings fehlen für eine derartige personalisierte molekularbiologische Analyse die entsprechenden Tools. Ziel unseres Projekts ist daher die Entwicklung eines personalisierten Tumormodells zur patientenindividuellen Wirkstofffindung und Anpassung der Krebstherapie. In einem ersten Schritt wurde hierzu die Kultivierung von Zelllinien in 2D-Kultur sowie in einem 3D-Modell getestet.

Methoden: Zur Etablierung eines innovativen Modells zur Kultivierung von humanen Tumorzellen wurden zunächst die Hepatomzelllinie $\mathrm{Hep}_{3} \mathrm{~B}$ und die Kolonkarzinomzellinie Caco-2 unter klassischen 2D-Kulturbedingungen kultiviert. Der Einfluss unterschiedlicher Medien sowie des Supplements Polyphosphat (polyP; dient als exogene Energiequelle und verhindert hypoxische Zustände in dreidimensionalen Zellclustern) wurde anhand von Zellzyklusanalysen untersucht Das Wachstum von Hep3B und Caco-2 auf dreidimensionalen Scaffolds wurde mittels Immunhistochemie sowie Immunfluoreszenzfärbungen evaluiert.

Ergebnisse: Der Zusatz von polyP-Materialien hatte keinen Einfluss auf das Kurzzeitüberleben von Caco-2 (3 Tage Kultur) und HepзB-Zellen (2 Tage Kultur) in klassischer 2D-Kultur. Hingegen war das Langzeitüberleben von Hep3B-Zellen in Gegenwart von Natrium-, Strontium- und Calcium-polyP gegenüber den Kontrollen verbessert. Die Vitalität von Caco-2- und Hep3B-Kulturen auf dreidimensionalen Scaffolds blieb über 10 Tage uneingeschränkt erhalten. Nach 17 Tagen Kul- tur auf dreidimensionalen Scaffolds wiesen Hep3B-Zellen zudem eine strukturiertere Anordnung auf als Kontrollzellen in 2D-Kultur.

Schlussfolgerung und Ausblick: Der Zusatz von polyP-Materialien verbessert das Langzeitüberleben von Zelllinien in 2D-Kultur. Dreidimensionale Scaffoldstrukturen sind geeignet zur Kultivierung von Zelllinien über bis zu 17 Tage. In weiteren Schritten wird nun getestet, ob eine Kultivierung primärer humaner Tumorzellen auf polyP-haltigen Scaffolds möglich ist.

\section{PS017}

The accumulation of skeletal muscle lipid species is gender-specific in an aging rat model for sarcopenic obesity

Thea Laurentius'; Robert Kob² ${ }^{2}$ Thomas Bertsch ${ }^{3}$; Cornel C. Sieber ${ }^{2}$; Mahtab Nourbakhsh ${ }^{4}$; Cornelius Bollheimer ${ }^{4}$

'Lehrstuhl für Altersmedizin, RWTH Aachen, Franziskushospital Aachen; ${ }^{2}$ Institut für Biomedizin des Alterns Friedrich-Alexander-Universität Erlangen-Nürnberg; ${ }^{3}$ Institut für Klinische Chemie, Laboratoriumsmedizin und Transfusionsmedizin - Zentrallaboratorium; Universitätsinstitut der Paracelsus Medizinischen Privatuniversität; ${ }^{4}$ Lehrstuhl für Altersmedizin Uniklinik RWTH Aachen

High-fat diet (HFD)-induced obesity results in adipose tissue saturation and excess deposits of fat in non-adipose tissues, such as skeletal muscles. This trend has been implicated in the age-related progressive loss of muscle mass, which is one of the main features of sarcopenia. The aim of this study was to differentiate lipid species that accumulate in the quadriceps muscles of Sprague-Dawley (SD) rats after 21 months of a HFD or control diet (CD). Here, we present a new procedure for quantitative lipid extraction from rat muscle tissue and further isolation of major ester-lipid classes, including free fatty acids (FFAs), cholesterol esters (CEs), triacylglycerols (TAGs), and glycerophospholipids (GPLs). Next, gas chromatography-mass spectrometry (GC-MS) was utilized to identify fatty acid methyl esters (FAMEs) within each individual lipid class. The data indicate that male and female rats accumulate distinct FAME species in their muscle tissues. The most prominent gender-related differences were found in the isolated TAG, GPL and FFA lipid classes. Independent of diet, however, male rats accumulated higher levels of octadecenoic and octadecadienoic acid, methyl esters in their muscle tissues.

This accumulation was significantly associated with impairments in muscle mass. Thus, greater deposits of octadecenoic and octadecadienoic acids in muscle tissue may serve as a novel indicator of the onset of age- or gender-related loss of muscle mass.

\section{PS018}

Sonographische Parameter als Prädiktoren der Progression der chronischen Nierenerkrankung - Langzeitdaten aus der CARE FOR HOMe Studie -

Claudia Simone Lennartz'; Sarah Seiler-Mußler²; Insa Emrich'; Kathrin Untersteller': John Pickering ${ }^{3}$; Danilo Fliser'; Gunnar H. Heine ${ }^{1}$

1 - Universitätsklinikum Homburg; ${ }^{2}$ - Nephrologische Praxis Saarlouis; ${ }^{3}$ Department of Medicine, University of Otago, Christchurch

Hintergrund: Um das Voranschreiten der chronischen Nierenerkrankung („,chronic kidney disease“; CKD) abzuschätzen, wird die Verwendung sonographisch bestimmbarer intrarenaler Widerstandsindices („resistive indices"; RI) diskutiert. Neben lokal-renaler Schädigung spiegeln intrarenale RIs (RRI) jedoch auch systemische Gefäßverän- 
derungen wider. Unsere Arbeitsgruppe entwickelte daher die DI-RISK ("difference of resistive indices of spleen and kidney") als neuen sonographischen Parameter mit dem Ziel systemische Störvariablen des RI zu eliminieren. In der CARE FOR HOMe Studie soll nun überprüft werden, ob sich die DI-RISK gegenüber den renalen RI als Prädiktoren der CKD Progression überlegen erweist.

Methoden: Zwischen 2008 und 2017 wurden in der fortlaufenden CARE FOR HOMe Studie 544 CKD Patienten in den GFR Kategorien $\mathrm{G}_{2}$ bis $\mathrm{G}_{4}$ eingeschlossen. Bei 491 dieser Patienten erfolgte eine duplexsonographische RI-Messung in drei Interlobararterien an der Rinden-Mark-Grenze in Ober-, Mittel- und Unterpol beider Nieren und bei 449 Patienten zusätzlich in drei Segmentarterien der Milz. Durch Subtraktion lienaler RIs von renalen RIs erhält man die DI-RISK. Klassische kardiovaskuläre Risikofaktoren wurden durch standardisierte Fragebögen erfasst. Als kombinierter renaler Endpunkt wurde eine eGFR Reduktion um > 50\%, Dialysepflichtigkeit und / oder Todesfälle jeder Genese definiert.

Ergebnisse: In univariablen Kaplan-Meier Analysen erweisen sich sowohl RRI $\geq 80$ (Log-Rank Test $p<0,001$ ) als auch eine DI-RISK $\geq 10$ (Log-Rank-Test $p<0,001$ ) als Prädiktoren des renalen Endpunktes. Nach Korrektur für die Störvariablen Alter, Geschlecht, eGFR („,estimated GFR") und Albuminurie bleibt der RRI $(\operatorname{Exp}(B) 1,064 ;[p<0,001]$; $95 \%$ KI $[1,035 ; 1,093])$, nicht aber die DI-RISK $(\operatorname{Exp}(B) 1,020 ;[p=0,309]$; $95 \% \mathrm{KI}[0,982 ; 1,059])$ unabhängiger Prädiktor des renalen Überlebens.

Schlussfolgerung: Entgegen unserer Hypothese erweist sich der unkorrigierte RRI dem korrigierten DI-RISK als Prädiktor des renalen Überlebens bei CKD Patienten als überlegen. Allerdings konnten wir in vorherigen Analysen zeigen, dass gegenüber dem etablierten Kidney Failure Risk Equation auch RRI keinen zusätzlichen ausgeprägten prognostischen Benefit erbringen.

\section{PS019 \\ Elektronische Stethoskope im Zeitalter von elektronischen Patientenakten}

Leander Edwin Melms; Tobias Müller; Andreas Jerrentrup; Tanja Nickolaus; Bernhard Schieffer; Jürgen Schäfer

Universitätsklinikum Marburg, Marburg;

Hintergrund: Seit dem Einsatz des Hörrohres durch René Laënnec in Frankreich vor gut ${ }^{200}$ Jahren nutzen wir in der Medizin Stethoskope zur Auskultation von physiologischen sowie pathophysiologischen Geräuschphänomenen. Im Vergleich zu den enormen Fortschritten der High-Tech-Medizin hat sich bei den Stethoskopen in den letzten Jahrzehnten nur wenig weiterentwickelt. Dies steht im Gegensatz zu innovativen Konzepten der Datenerfassung und Auswertung im Zeitalter von eHealth und elektronischen Patientenakten (ePA). Derzeit erfassen ePA lediglich Text und Bildbefunde. Die Übernahme von Befunden der Herz-, Lungen- und Abdominalauskultation setzt eine entsprechende technische Ausstattung voraus. Ein ePA-kompatibles elektronisches Stethoskop könnte diese Datenerfassung ermöglichen.

Gegenstand der Studie: Ziel unseres Vorhabens ist die Entwicklung eines ePA-fähigen Stethoskops mit automatischer Befundanalyse. Durch die digitale Erfassung von Auskultationsbefunden unmittelbar im Notfall- bzw. Praxisbereich und die Speicherung in ePA-Systeme wird der Befund wiederholt objektivierbar und es könnte auch die diagnostische Qualität verbessert werden. Hiermit bieten sich neuartige Möglichkeiten der studentischen Lehre an sowie innovative Ansätze der Datenanalyse. Die erfassten Geräuschphänomene werden durch Deep-Learning-Algorithmen automatisch auf physiologisch bzw. pathologisch überprüft. Bei suspektem Klangbild kann eine zielgerichtete weiterführende apparative Diagnostik automatisch angefordert werden.

Methode: Unser System besteht aus einem Kondensatormikrofon, einem Vorverstärker- und einem Tiefpassfilter-Schaltkreis und wird direkt an ein handelsübliches Stethoskop angeschlossen. Die Ausgabe des analogen Signals zur automatischen Befundanalyse erfolgt drahtlos in Echtzeit an ein Smartphone. Dieses ist mit einer selbstentwickelten Applikation ausgestattet und gibt die Verdachtsdiagnose aus. Die Hardware-Kosten liegen im Bereich von $20,00 €$ und erlauben so eine breite Anwendung in der Klinik aber auch in der Heim-Überwachung z.B. bei Risikopatienten.

Ergebnisse: In einer präklinischen Prüfphase wird derzeit die diagnostische Qualität unseres Deep-Learning-Modells anhand der 3126 hinterlegten Herzauskultationen der Physionet Challenge 2016 evaluiert. Die Detektion der Geräusche ist dabei exzellent und Störgeräusche können verlässlich herausgefiltert werden. Die Sensitivität des Algorithmus beträgt $80,53 \%$ und die Spezifität $87,66 \%$. Die Einbindung der digitalen Datensätze in ePA-Systeme ist problemlos und anwenderfreundlich möglich.

Zusammenfassung: Die elektronische Erfassung von Herz- und Lungengeräuschen erlaubt eine automatisierte Erkennung von Vitien. Durch die Aufzeichnung dieser Geräusche in einem standardisierten Setting bereits im Ambulanzbereich können Veränderungen der Geräusche (wie z.B. bei Endokarditis oder bei mechanischen Klappen) nicht nur besser archiviert und dokumentiert, sondern auch im Verlauf verlässlich überwacht werden.

\section{PS020}

\section{Medizinische Versorgung in Afghanistan}

Azim Mosafer'; Cornel Eisfeldt ${ }^{2}$

'ADAV-Weimar e.V., Weimar; ${ }^{2}$ Universitätsklinikum Magdeburg A.ö.R., Magdeburg

Problemstellung: Afghanistan, das Land am Hindukusch, befindet sich seit dem Jahr 2002 im Wiederaufbau. Nach dreißig Jahren Krieg und aktuellen Konflikten kommt das Land nicht zur Ruhe. Die medizinische Ausbildung und Versorgung ist unbefriedigend. Die Landfläche beträgt $652.090 \mathrm{~km}^{2}$ (Deutschland $357.376 \mathrm{~km}^{2}$ ). Die Gesamtbevölkerung wurde 2017 auf 36,8 Millionen geschätzt, davon leben ca. 80 $\%$ auf dem Land und $20 \%$ im städtischen Bereich, Bevölkerung unter 15 Jahre $46,3 \%$, Bevölkerung im Alter von 15-64 Jahren 51,5\%, im Alter ab 65 2,2\% (2010). Die Amtssprachen sind Dari und Paschtu. Die Sicherheitslage und die Landflucht sind aktuelle Probleme des Landes. UNESCO beziffert die Alphabetisierung der Afghanen >15 Jahre von 18\% (2000). Lebenserwartung bei der Geburt (m) 44,0 (2008) 62(2015), (w) 43,9 (2008) 64,6(2015). Geburten pro Frau: 4,8 Kinder (2015). Ärzte Dichte: für-10.000 EW 3 Ärzte (2014). Krankenhausbetten: für 10.00o EW 4 Betten (2010). Festnetzverträge $0,3 / 100$ EW. Internetnutzer 10,6/100 EW Mobile Breitband-Internetverträge 14,3 / 100 EW. Mobilfunkverträge 66/100 EW (2016). Laut Grundgesetz in Afghanistan ist die medizinische Versorgung kostenfrei. Die Behandlung in den med. Einrichtungen und die Privatisierung der med. Versorgung und Ausbildung ist sehr Insuffizient. Diese erzeugen einen Verlust der Autorität und Glaubwürdigkeit der Ärzte. Die wohlhabenden Patienten suchen Heilung im Ausland (DW). Täglich fliegen ca. tausend Afghanen nach Indien. Etwa $70 \%$ sind Gesundheitstouristen. Dies ist ein volkswirtschaftlicher Verlust von rund 240 Millionen USD. Trotz großer Hürden floriert der Gesundheitstourismus. (DW). 
Ziele des Vereins: Seit der Gründung im Jahr 2004 hat der Afghanisch - Deutsche Ärzteverein in Weimar u.a. fünf größere Projekte in Afghanistan initiiert. Zwei davon sind: 1. E-Learning ( medical education) in Zusammenarbeit mit der Fa. Lecturio. Hierbei werden medizinische Vorlesungen via E-Learning ab dem ersten Semester angeboten. Das ein jährige Pilotprojekt läuft seit dem Sommersemester 2017

Problem: Internetzugang ist für Studierende sehr teuer. Sprachbarriere- ca. 50\% der Studenten und Lehrkräfte haben Englischkenntnisse.

Lösung: Hotspot in den Universitäten. Untertitel in den Landessprachen

Telemedizin (TM): ADAV-Weimar e.V. initiierte 2010 das Projekt „Afghan-German-Telemedizin". Die entsprechend der afghanische Infrastruktur entwickelte Software wurde nach einem landesweite Pilotprojekt 2014 nun im Jahr 2017 mit 6 TM-Zentren in den Provinz erweitert. Seit 2011 konnten 1308 Patienten via TM behandelt werden. Nur 98 orthopädische Patienten(ca. 10\%) wurden durch das entsandte Ärzteteam operiert. Die Datenanalyse zeigt, dass der Großteil (ca. $80 \%$ ) der TM-Patienten nach Diagnostik konservativ behandelt wurden.

Zusammenfassung: Die TM / E-Learning ermöglichten eine notwendige medizinische Versorgung / Ausbildung, da geographische und gesellschaftliche Hindernisse überwunden werden. Trotz großer Entfernung wird effiziente Hilfe geleistet.

\section{PS021}

Persistierende Gewichtsreduktion bei Übergewicht und Adipositas durch ein innovatives nicht-invasives Medizinprodukt und Beeinflussung des individuellen adipositas-assoziierten Risikos

Aleksandra Nowak'; Pia Hüttemann'; Jessica Königsmann; Peter von Seck²; André Schmidt-Lucke ${ }^{2}$; Caroline Schmidt-Lucke

${ }^{1}$ MEDIACC GmbH; ${ }^{2}$ Praxis für Innere Medizin

Hintergrund: Die Gewichtsreduktionsprogramme bei Adipositas scheitern meist an der im Anschluss eintretenden erneuten Gewichtszunahme. Das Ziel dieser Arbeit war es daher die Hypothese zu untersuchen, ob ein neues intraorales nicht-invasives Medizinprodukt bei Nutzung zu jeder Mahlzeit, über eine Kauflächenreduktion, zu einer Gewichtsreduktion bei Patienten mit krankhafter Adipositas führt und ob das reduzierte Gewicht auf Dauer aufrechterhalten werden kann.

Methoden: Hierzu wurden im Rahmen einer prospektiven, randomisierten, kontrollierten (Wartekontrollgruppe), einfach verblindeten, multizentrischen Pilot-Studie 35 übergewichtige Patienten mit mindestens 2 frustranen Abnehmversuchen rekrutiert (29 Frauen, 6 Männer, BMI: 32,2 [29,7 - 37,8]; Alter 47 Jahren [28 - 56]). Die Patienten trugen die Zahnschienen über einen Zeitraum von 3 Monaten zu jeder Mahlzeit und wurden in der Nachbeobachtungsphase von insgesamt 12 Monaten weiter begleitet, ohne die Schiene tragen zu dürfen. Während der Studie sollten sie keine Diät halten, essen was sie mögen und bis sie satt werden und keine Kalorien zählen.

Ergebnisse: Durch die Zahnschiene erzielten die Patienten einen signifikanten Gewichtsverlust von initial 87,6 kg [80,5 - 101,3] um 1,5 kg $[-2,6$ bis $-0,4](p=0.008)$ nach 1 Monat und nahmen weiter bis zum Ende der Tragedauer nach 3 Monaten um insgesamt 2,7 kg (-4,6 bis $-0,8, p=0,003) a b$. Während der Wartekontrollzeit von 4,5 Monaten vor Schienenerhalt blieb das Körpergewicht der Patienten jeweils un- verändert. Im intraindividuellen Vergleich nahmen sie nachfolgend nach 3 Monaten mit Schienenbenutzung signifikant $a b(p=0,011)$. Dies ist der Beweis dafür, dass es sich bei der Schienenbenutzung nicht um einen Placeboeffekt durch das Programm handelt. AnschlieBend hielten die Patienten ihr Gewicht über 12 Monate $(p=0.023)$. Über $73 \%$ der Patienten würden die Schiene weiterempfehlen bzw. $76,5 \%$ hätten die Schiene gerne über längere Tragedauer gehabt, je unabhängig von dem Ausmaß der Gewichtsreduktion. Weder Einschlussort noch sozioökonomischer Status hatten einen signifikanten Einfluss auf das Ausmaß der Gewichtsreduktion. Es traten keine relevanten unerwünschten Nebenwirkungen auf. Ursächlich für die Gewichtsreduktionen ist am ehesten die Modifizierung des Essverhaltens (Verlängerung der Mahlzeitdauer, Essen bis Sattwerden, weniger Zwischenmahlzeiten, $\mathrm{p}<0.0007$ ).

Fazit: Dieses ist das erste nicht-invasive Medizinprodukt, das eine Gewichtsreduktion über eine Veränderung der Essgewohnheiten bewirkt. Besonders hervorzuheben ist, dass die Patienten bis zum Sattwerden essen durften und nachfolgend ihr Gewicht über 12 Monaten hielten. Somit wirkt die Schiene sogar über die eigentliche Tragedauer hinaus. Dieses Medizinprodukt stellt daher eine Alternative für bisher bekannte Gewichtsreduktionsprogramme dar und diese wird in einer größeren Folgestudie verifiziert.

\section{PS022 \\ PGE2-dependent MEF2-activation in an inflammatory cardi- omyopathy}

Richard Schell; Philipp Theis; Clemens Haslinger; Felix Alban; Hugo A. Katus; Johannes Backs

Universitätsklinikum Heidelberg, Heidelberg;

Rationale: Despite heart failure's striking prevalence, mortality and morbidity the underlying molecular mechanisms and their differences in distinct etiologies still remain unclear. Recent findings suggest that inflammation and in particluar Prostaglandin E2 (PGE2) plays a critical role for the ventricular remodeling and progression of heart failure. The myocyte enhancer factor 2 (MEF2) is a crucial inductor of pathologic cardiac remodeling. In a recent in vitro study, we could show that PGE2 leads to a strong MEF2-activation. Furthermore the investigation provides data that $\mathrm{PGE} 2$ stimulates via $\mathrm{EP}_{3}$-receptor an intracellular signal transmission, which drives protein kinase $D(P K D)$-dependent hyperphosphorylation of histone deacetylase 5 (HDAC5), resulting in nucleo-plasmatic shuttling of $\mathrm{HDAC}_{5}$ and increased MEF2 activity. The aim of the present work was to translate these findings into the in vivo situation and to study their functional relevance in a LPS-induced sepsis model as a model for an inflammatory cardiomyopathy.

Results: Treating BALB/c-mice with a weight-depending dose $(7 \mathrm{mg} /$ $\mathrm{kg}$ body weight) of Lipopolysaccharide (LPS from Escherichia coli O111:B4) leads to severe depression of (left-) ventricular function and acute heart failure. We could assess a time-dependent and significant decrease of ejection fraction (EF) by transthoracic echocardiography. The resulting setting of inflammation comes along with considerably increased levels of PGE2 in the myocardium and its forming enzymes Cyclooxygenase 2 and Prostaglandin-E-synthase 1. Furthermore we see an increase of catalytic PKD activity and a significant hyperphosphorylation of $\mathrm{HDAC}_{5}$ at Ser-259. As a key finding, this inflammatory model leads to a clear activation of MEF2, which could be shown by a myocardial $\beta$-Galactosidase-staining in LPS-treated MEF2-lacZ reporter mice. Moreover, mRNA levels of the MEF2 target genes including Myomaxin and $\mathrm{CCL}_{3} / \mathrm{CCL} 6$ represent the induction of cardiac remodeling genes. 
Conclusion: Inflammation and its response seem to play crucial roles in the induction of ventricular remodeling and progression of heart failure. With these in vivo data, we provide evidence that cardiac inflammation under the condition of a septic disease induces activation of MEF2 and its potential upstream signaling way consisting of PGE2, $\mathrm{EP}_{3}, \mathrm{PKD}$ and $\mathrm{HDAC}_{5}$. The described pathway may provide a new link between cardiac inflammation and the initiation and progression of remodeling and heart failure. Further loss-of-function of the single components of the described pathway are on way to investigate their relevance in the different inflammatory etiologies of remodeling. These studies may provide new therapeutic targets to treat heart failure caused by inflammatory injuries.

\section{PS023 \\ Radikale Pankreaschirurgie bei Malignomen mit Gefäßbeteili- gung}

Veronika Scholtz'; Hans-Urlich Schulz² ; Zuhir Halloul'; Frank Meyer ${ }^{1}$

${ }^{1}$ Universitätsklinikum Magdeburg A.ö.R., Magdeburg; ${ }^{2}$ AMEOS-Klinikum Haldensleben, Haldensleben

Das Pankreaskarzinom(Ca) zeigt nicht selten Gefäßadhärenzen/-infiltrationen, was insbesondere portalvenös nicht zwangsläufig Irresektabilität bedeuten muss.

Patienten/Methodik: In einem hochspezialisierten universitär-chirurgischen Zentrum wurden über einen definierten Untersuchungszeitraum alle konsekutiven Fälle mit Gefäßersatz bei Pankreasresektionen einer retrospektiven Auswertung im Rahmen einer unizentrischen Observationsstudie unterzogen. Verglichen wurden Patienten-, Lokalbefund- und Behandlungs-assoziierte Aspekte wie Alters-/Geschlechtsstruktur, präoperatives Diagnostikprofil, viszeralchirurgische Operationsverfahren, $\mathrm{R}^{\circ}$-Resektionsrate, gefäßchirurgische/-medizinische Therapiemaßnahmen sowie früh-postoperatives (Krankenhausverweildauer; Morbidität/Letalität) und onkochirurgisches Outcome (5-Jahres-Überlebensrate).

Ergebnisse: Insgesamt wurden über 10 Jahre 34 Patienten (20 Männer/14 Frauen; Durchschnittsalter: 59 Jahre) ermittelt: Pankreas-Ca: $\mathrm{n}=27$; Pankreaskorpus-Ca: $\mathrm{n}=3$; Pankreasmetastase: $\mathrm{n}=2$; Pankreas-Caput-Corpus-Ca/Klatskintumor: $\mathrm{n}=\mathrm{je} 1$.

Die Patientenmehrzahl wurde mit der Pylorus-Preserving Pancreatic Head Resection(PPPHR) nach Traverso/Longmire versorgt. Weiterhin kamen die Kausch-Whipple-Op, die (sub-)totale Pankreatoduodenektomie und die Pankreaslinksresektion zum Einsatz.

Am häufigsten wurde eine Infiltration der Pfortader diagnostiziert, gefolgt von V. mesenterica superior, A. hepatica, V. mesenterica inferior, Milzgefäßen, A. gastroduodenalis, A. mesenterica superior, A. colica media und Mesenterialwurzel bzw. V. cava inferior.

Je nach tumorinfiltrierend betroffenem Gefäßabschnitt wurde die Mitresektion vorgenommen und die Kontinuität mittels Rekonstruktion durch Naht (Re-Anastomosierung mittels direkter Naht) oder Gefäßersatz (PTFE-Prothese, V.-saphena-Interponat, autologer Venenpatch) wieder hergestellt.

$56 \%$ der Patienten wurden Ro-reseziert. Die perioperative Morbidität betrug 50\%, die Hospitalletalität 5,8\%. Die mediane Überlebenszeit wurde mit 22 Monaten ermittelt. Die 5-Jahres-Überlebensrate lag bei $14 \%$.

Schlussfolgerung: Trotz tumorbedingter Gefäßalteration ist das onkochirurgische Langzeit-Outcome ansprechend, was unter vertretbarer Risiko-Nutzen-Relation das aufgeführte Vorgehen rechtfertigt.
Die interdisziplinäre Kombination eines onkochirurgisch versierten und eines Gefäß-Chirurgen ist ein optimaler Ansatz für die Operation bei ausgewählten Patienten mit Gefäßinfiltration beim Pankreas-Ca. Die venöse Infiltration mit konsekutiver En-bloc-Resektion wird mittlerweile als eine sichere Prozedur mit akzeptablem perioperativen $\mathrm{Ri}$ siko und Outcome unter kurativer Intention betrachtet. Die arterielle Gefäßresektion stellt jedoch beim Pankreas-Ca immer noch eine Ausnahme dar. Als mögliche Option kann sie unter Einbeziehung eines neoadjuvanten Konzeptes in Betracht gezogen werden kann, wenn ein tumorfreier Resektionsrand in sorgfältig selektierten Patienten erreicht werden kann.

\section{"Young investigators - the future is now" III}

\section{PS024}

\section{The effect of microgravity on central aortic blood pressure}

Felix S. Seibert; Ulrik Stervbo-Kristensen; Nikolaos Pagonas; Benjamin Rohn; Frederic Bauer; Nina Babel; Timm Henning Westhoff

Marien Hospital Herne, Universitätsklinik Ruhr-Universität Bochum, Herne

Background: Blood pressure has been traditionally measured at peripheral arteries. In the past decade evidence has grown, that central aortic blood pressure may be a more powerful predictor for cardiovascular events, but data on its regulation are rare. The present works examines the impact of microgravity on central blood pressure for the first time.

Methods: We performed seven parabolic flights in Bordeaux, France, with 22 seconds of weightlessness in each parabola $(n=620)$. Hemodynamic parameters including central systolic blood pressure were measured non-invasively in a free-floating position using the oscillometric Mobil-O-Graph (I.E.M., Stolberg, Germany) in 20 healthy subjects (1943 years of age). RESULTS: Arterial elasticity at rest was normal in all participants (augmentation index 14\% [IQR 10-22], pulse wave velocity $5.2 \mathrm{~m} / \mathrm{s}$ [IQR 5.0-5.4]). Transition of $1 \mathrm{~g}$ to o $\mathrm{g}$ led to a significant increase of central systolic blood pressure from 124 (interquartile range [IQR] 118-133) to 127 (IQR 119-133) $\mathrm{mmHg}(\mathrm{p}=0.017)$. Cardiac index propelled from 2.5 (IQR 2.2-2.8) to 2.7 (IQR 2.3-3.0) I/ $\mathrm{min} / \mathrm{m}^{2}$ (po.05).

Conclusion: Whereas there is a multitude of studies on the effects of microgravity on peripheral blood pressure, this study provides first data on central aortic blood pressure. An acute loss of gravity leads to a central blood volume shift with an augmentation of cardiac output. In healthy subjects with normal arterial stiffness the compensatory decrease of peripheral resistance does not outweigh this effect resulting in an increase of central blood pressure. 


\section{PS026 \\ Hyperosmolarer Stress potenziert die TRAIL-Sensitivität bei kolorektalen Karzinomzellen}

\author{
Simon Sirtl; Martin Ehrenschwender
}

Universitätsklinikum Regensburg, Regensburg; Hintergrund: Aktivierung von sog. Todesrezeptoren kann in Tumorzellen regulierte Zelltodmechanismen wie Apoptose initiieren, was auch therapeutisch genutzt werden kann. Zudem konnte für den Todesliganden TRAIL die selektive Wirksamkeit in Tumorzellen ohne' Kollateralschäden' im gesunden Gewebe gezeigt werden. Klinisch sind bislang die Erfolge TRAIL-basierter Therapien allenfalls moderat, da viele Tumorentitäten Apoptose resistent sind. Neben zellintrinsischen Faktoren spielen hierbei auch physiko-chemischen Faktoren der Tumorumgebung eine wichtige Rolle. Ziel der Studie war es zu untersuchen, wie sich die Modulation des osmotischen Drucks auf die Wirksamkeit von TRAIL in kolorektalen Karzinomzellen auswirkt.

Methodik: Die humane kolorektale Karzinomzelllinie HCT116 wurde mit TRAIL in An- und Abwesenheit von Natriumchlorid als, osmotischen Stressor' stimuliert. Das Zellüberleben wurde in MTT-basierten Viabilitätsassays und in der Durchflusszytometrie (Annexin-V/7-AAD Färbung) erfasst.

Ergebnisse: In Gegenwart von $\mathrm{NaCl}$ war die TRAIL-induzierte Apoptose deutlich gesteigert ( $80 \%$ vs. $46 \%$ ), wobei die Zytotoxizität von $\mathrm{NaCl}$ allein vernachlässigbar war. Als zugrundeliegender Mechanismus konnte eine verstärkte Amplifikation des von den TRAIL-Todesrezeptoren ausgehenden Stimulus über den mitochondrialen Apoptoseweg herausgearbeitet werden.

Ausblick: Diese Arbeiten zeigen dass die Sensitivität von Tumorzellen gegenüber Todesrezeptor-induzierter Apoptose durch Modulation der Tumorumgebung beeinflusst werden kann. In soliden Tumoren könnte dies auch therapeutisch nutzbar sein.

\section{PS027 \\ Die Rolle des extrazellulären Kalziums bei Adipositas-induzier- ter Inflammation}

Miriam Sommer; Stephan Thrum; Nora Raulien; Manuela Rossol; Matthias Blüher; Ulf Wagner

\section{Universitätsklinikum Leipzig AöR}

Obwohl Adipositas als metabolische Erkrankung weltweit längst zu einem ernsten Problem öffentlicher Gesundheit geworden ist, bleiben viele Details ungeklärt. So geht die Entwicklung einer Adipositas im viszeralen Fettgewebe mit Migration von Leukozyten einher, die im Gewebe v.a. durch Aktivierung von Makrophagen mit einer chronischen Entzündung assoziiert ist. Dies ist Grundlage der Entwicklung weiterer metabolischen Krankheiten wie Typ 2-Diabetes und Erkrankungen des kardiovaskulären Systems. Es konnte gezeigt werden, dass extrazelluläres Kalzium (exCa2+) durch Aktivierung des $\mathrm{NLRP}_{3}$ Inflammasoms den Immunzellen als Warnsignal und Verstärker ihrer Aktivierung dient und so maßgeblich zu Entzündungsprozessen beiträgt. Die genauen Mechanismen jedoch, die exCa2+ bei der Adipositas-induzierten Inflammation spielt, sind bisher nicht ausreichend verstanden.

Deshalb wurden hier Proben von Fettgewebe und aus Monozyten differenzierte Makrophagen (MDM) von adipösen Patienten im Vergleich mit schlanken Individuen untersucht. Durch Reifung mit autologem Serum wurden MDM kultiviert, die ein vitro-Modell zur Bestimmung Probanden-spezifischer Makrophagen-Eigenschaften ermöglichen. Unter inflammatorischen Bedingungen und Stimulation mit exCa2+ zeigten die MDM in allen Versuchen eine vermehrte, dosis- und patientabhängige IL-1 $\beta$-Sekretion. Gleiche Beobachtungen ließen sich in Fettgewebskulturen machen, wobei höhere Zytokinspiegel im viszeralen als im subkutanen Fettgewebe nachweisbar sind. Weiterführend lässt sich beobachten, dass trotz exCa2+-Zugabe weniger IL-1 $\beta$ sezerniert wird, wenn eine Hemmung des G-Protein-gekoppelten calciumsensitiven Rezeptors (CaSR) erfolgte. CaSR konnte in MDM nachgewiesen werden, wohingegen GPRC6A eine weniger wichtige Rolle zukommt. Unter Hypoxie ließ sich eine Sensitivierung der MDM gegenüber exCa2+ zeigen und kann damit Hinweise geben auf die proinflammatorische Wirkung von Gewebshypoxie bei Adipositas. In einer prospektiven, klinisch-experimentellen Studie sollen nun die Zytokin-Sekretion, Subpopulationen und CaSR-Expression der MDM, sowie Klinik bei Patienten mit inflammatorischer Adipositas untersucht und mit einer normalgewichtigen Kohorte verglichen werden. So lässt sich überprüfen, ob die in vitro vorhandene, inflammatorische Wirkung von Kalzium auf die Klinik übertragbar ist. Nach Angaben der WHO hat sich die Zahl adipöser Menschen seit 1975 nahezu verdreifacht. Neue prognostische Marker und therapeutische Optionen werden dringend gebraucht. Durch ein neues pathophysiologisches Verständnis suchen wir dies zu erreichen und so die Inflammation-vermittelte Morbidität bei adipösen Patienten zu senken.

\section{PS028}

Utility of Neutrophil-Lymphocyte Ratio, Platelet-Lymphocyte Ratio and Mean Platelet Volume as diagnostic and prognostic markers in patients with Hepatocellular carcinoma, Prostate carcinoma, Stomach carcinoma, Aplastic Anemia

V.S Srikanth; M.G.K Pillai

Amrita Institute of Medical Sciences, $\mathrm{KOCHI}$, India; Amrita Institute of Medical Sciences, Kochi, India

Objective: To study the potential role of NLR, PLR, MPV, MPV/Platelet ratio as diagnostic and prognostic markers in patients with HCC, Prostate cancer, Stomach cancer, Aplastic anemia. Methods: We have conducted the present study by screening 2,08,486 patients who have got admitted during the period January 2013- June 2017 as in patients in AIMS, Kochi. The data collected were analyzed for Neutrophil-Lymphocyte Ratio, Platelet-Lymphocyte Ratio, MPV, MPV/Platelet ratio. Inclusion criteria: Patients admitted with a diagnosis of $\mathrm{HCC}$, prostate cancer, stomach cancer and aplastic anemia irrespective of the age group. Exclusion criteria: Patients with other comorbidities, more than 1 malignancy, presence of secondary infection, any source of sepsis. The data was collected and analyzed on windows excel Results: NLR and MPV/Platelet ratio were highly significant in HCC, Prostate and stomach cancer where as PLR was significant in Prostate cancer, MPV was significant in Stomach carcinoma. These ratios were not significant in aplastic anemia. Conclusion. From our study, we conclude that NLR, PLR are better cost-effective predictor and prognostic markers of with HCC, Prostate cancer, Stomach cancer. These ratios can be used at the primary health care level as it can be derived from a simple $\mathrm{CBC} /$ Peripheral smear. This will aid in early identification and management of liver abscess even in rural areas of developing country like India. 


\section{PS029}

Eine Sarkopenie und eine Reduktion des viszeralen Fettgewebes weist auf Komplikationen im Verlauf bei Patienten mit Colitis ulcerosa, aber nicht mit Morbus Crohn, hin.

Tina Stahr'; René Aschenbach ${ }^{2}$; Thomas Lehmann ${ }^{2}$; Tony Bruns ${ }^{2}$; Andreas Stallmach ${ }^{2}$

${ }^{1}$ Friedrich-Schiller-Universität, Universitätsklinikum Jena, Jena; ${ }^{2}$ Universitätsklinikum Jena, Jena

Einleitung: Die Einschätzung des Krankheitsverlaufs bei chronisch-entzündlichen Darmerkrankungen ist von großer Bedeutung. So ist das Abschätzen von Komplikationen vor operativen Eingriffen bei der Indikationsstellung und Festlegung der Operationsstrategie sehr wichtig. In Studien bei Traumapatienten oder Patienten mit Leberzirrhose konnte gezeigt werden, dass Sarkopenie mit Komplikationen assoziiert ist $(1,2)$. Wir untersuchten deshalb die prädiktive Bedeutung der Sarkopenie (Muskelvolumens des Psoas, des M. quadratus lumborum und der autochthonen Rückenmuskulatur) und des viszeralen Fettgehaltes bei Patienten mit Morbus Crohn (MC) und Colitis ulcerosa $(\mathrm{CU})$.

Methodik: Es wurden bei 53 Patienten mit MC und 20 Patienten mit CU Computertomographien des Abdomens hinsichtlich des Muskelvolumens auf Höhe des 3. Lendenwirbelkörpers und des Volumens des viszeralen Fettgewebes auf Bauchnabelhöhe untersucht. Der weitere Verlauf wurde über 12 Monate durch Aktenauswertung analysiert. Endpunkte waren die Hospitalisierung, Operation und Operation mit Komplikationen.

Ergebnisse: Von den 53 Patienten mit MC wurden bei 20 Patienten keine Komplikationen und bei 33 Patienten mindestens eine Komplikation beobachtet ( 9 Hospitalisierungen, 11 Operationen, 13 Operationen mit Komplikationen). 9 der 20 CU-Patienten hatten einen unkomplizierten Verlauf, bei 11 Patienten ist mindestens eine Komplikation aufgetreten (2 Hospitalisierungen, 5 Operationen, 4 Operationen mit Komplikationen). Das Muskelvolumen (VM) zeigte bei CU mit zunehmender Schwere der Komplikationen eine Abnahme; diese Beobachtung fand sich nicht bei MC. CU-Patienten, die eine Operation mit Komplikation hatten, wiesen im Vergleich zu denen ohne Ereignis ein signifikant reduziertes VM (Median(IQR): $298 \mathrm{~cm}^{3}(72)$ vs. $417 \mathrm{~cm}^{3}(148)$, $\mathrm{p}=0,02$ ) auf.

Das Volumen des viszeralen Fettgewebes (VVF) bei MC und bei CU zeigte von den Patienten ohne Ereignis hin zu denen mit Hospitalisierung, Operation und Operation mit Komplikation einen signifikant fallenden Trend. Im Vergleich zu CU-Patienten ohne Ereignis wiesen sowohl CU-Patienten mit Operation (Median(IQR): $318 \mathrm{~cm}^{3}(145)$ vs. $1084 \mathrm{~cm}^{3}$ (540), $p=0,001$ ) als auch CU-Patienten mit Operation und Komplikation (Median(IQR): $147 \mathrm{~cm}^{3}(180)$ vs. $1084 \mathrm{~cm}^{3}(540), p=0,003$ ) ein signifikant reduziertes VVF auf.

Diskussion: Ein geringeres viszerales Fettgewebe bei Colitis ulcerosa geht mit dem Auftreten von Hospitalisierung, Operation und Operation mit Komplikation einher. Eine prädiktive Bedeutung der Sarkopenie konnte bei Patienten mit MC für den weiteren Krankheitsverlauf nicht nachgewiesen werden.

1) van Vugt, J.L.A. et al. Journal of Hepatology, 2017, DOI: https://doi.org/10.1016/j.jhep.2017.11.030

2) DeAndrade, J. et al. Journal of Surgical Research, 2018 (221): 161-166.

\section{PS030}

Anspruchsvolle Diagnosefindung im Falle einer akuten Appendicitis bei intestinaler Malrotation

\author{
Emilia Strungaru; Cora Wex; Frank Meyer
}

Universitätsklinikum Magdeburg A.ö.R., Magdeburg

Ziel: Mittels wissenschaftlichem Fallbericht soll - basierend auf ausgewählten Referenzen der Literatur und den gewonnenen Erfahrungen im kasusspezifischen diagnostischen und therapeutischen Management - der ungewöhnliche, jedoch im Alltag nicht ausgeschlossene und vorkommende Fall einer akuten Appendicitis bei Malrotation des Darms exemplarisch dargestellt werden.

Fallpräsentation: Ein 54-jähriger, männlicher Patient klagte seit $7 \mathrm{~d}$ über "unterschwellige“ Beschwerden, im Mittelbauch beginnend mit Besserung nach Wasserlassen, am Vortag deutlich zunehmende Schmerzen im medialen/linken Unterbauch, aktuell seit $30 \mathrm{~min}$ akut eingesetzter kolikartiger Schmerz, Aggravierung in gebeugter Haltung, letzter Stuhlgang vor Selbstvorstellung (kein[e] Diarrhoe, Obstipation, Übelkeit, Erbrechen, Fieber, Algurie - Nebendiagnosen: saisonale allergische Rhinitis, Asthma bronchiale, Allergie: Pollinosis; Medikation: Berotec N 100 Mg, Ibuprofen 400 mg b.B. [1-2x/Woche]). Klinisch (182 cm, 78 kg) war der Patient wach, kooperativ, Zeit-/Ort-/ Situation-/Person-orientiert (GCS: 15, Cor und Pulmo ohne pathologischen Befund). Am Abdomen bestand ein deutlicher Druckschmerz mit punctum maximum im li UB, ubiquitärer Klopfschmerz mit Ziehen in den li UB, Abwehrspannung im li UB (Nierenlager frei, DG reduziert in allen 4 Quadranten, digital-rektale Untersuchung unauffällig, Prostata etwas vergrößert). Es wurde der V.a. eine Sigma-Diverticulitis erhoben. Das Labor (SI) zeigte L: 9,1; CrP: 79,8; Urin: 9 Ery's. Die transabdominelle Sonografie äußerte ebenso den V.a. Diverticulitis (Rö-Abdomenübersichtsaufnahme im Stehen keine freie Luft, keine lleuszeichen). Das CT-Abdomen erhob die Diagnose der akuten Appendicitis im li Mittel-/Unterbauch bei malrotiertem Colon ascendens mit geringen Mengen freier Flüssigkeit im kleinen Becken (keine freie Luft). Es wurde die OP-Indikation gestellt: Es erfolgte die offene lleozökalresektion mit Seit-zu-Seit-lleo-Ascendostomie bei einer intraoperativ vorgefundenen schweren ulcero-phlegmonösen perforierten Appendicitis mit schwerer abszedierender Periappendicitis und ausgedehnter fibrinös-eitriger Serositis. Im postoperativen Verlauf rezidivierendes, sich im Verlauf besserndes Erbrechen, zögerliche, jedoch erfolgreiche Mobilisation und Kostaufbau, substitutionspflichtige Hypokaliämie, Drainageentfernung am 5. Tag, Normalisierung der Infektparameter, Klammernahtmaterialentfernung am 10. Tag (keine allgemeinen/spezifischen Komplikationen - Entlassung am 12. Tag).

Chirurglnnen und assoziierte (initialbetreuende und bildgebend tätige ärztliche) Fachkollegen sollten sich über die vielfältigen Lagevarianten der Appendix vermiformis bewusst sein, die im Falle einer akuten Appendicitis bei klinisch bestehendem akutem Abdomen die Differenzialdiagnose schwierig gestalten, die Diagnosefindung erheblich erschweren und nicht zuletzt die Entscheidungsfindung zu Op-Technik und -Taktik (laparoskopisch vs. offen, Zugangsweg/-lokalisation etc.) beeinflussen können. 


\section{PS031}

\section{Mucosal Plasmablasts as biomarkers in Systemic sclerosis}

Georg Tilgner'; Axel Schulz²; Qingyu Cheng ${ }^{2}$; Jonas Martin²; Claudia Kedor'; Falk Hiepe'; Bimba Franziska Hoyer ${ }^{3}$

${ }^{1}$ Charité Universitätsmedizin Berlin; ${ }^{2}$ Deutsches Rheuma-Forschungszentrum Berlin (DRFZ); ${ }^{3}$ Universitätsklinik Schleswig-Holstein (UKSH)

Background: Systemic sclerosis (SSc) is a rare connective tissue disease affecting the skin and i.e. the lung and gastro-intestinal tract (GI). Therapy is difficult and biomarkers for pulmonary or Gl-involvement are lacking today. B cells and plasmablasts (PBs) play a major role in patients with Systemic Sclerosis. This finding is reflected in the presence of autoantibodies against CENP and Scl-70. Furthermore B Cell depleting therapy seems to be effective. Among others, Mei et al. revealed that a major part of human steady-state blood plasma cells have their origin in mucosal immune reactions, determined by their specific expression of IgA, CCR10 and $a_{4} \beta 7$-Integrin - markers for mucosal origin (Mei et al 2009). Based on those markers, the central aim of our study is to put alterations of the mucosal immune systems into perspective with clinical symptoms of SSc patients.

Methods: 49 SSc patients ( 25 with limited Systemic Sclerosis and 20 with diffuse Systemic Sclerosis) were analysed by flow cytometry and compared to 23 healthy donors. The flow cytometry staining markers used to evaluate our hypothesis of over activated $B$ cells in SSC are CD19, CD20, CD27, IgM, CD95, IgD, CD138, HLA-DR, IgA, CCR7, CCR10, $\beta 7$-Integrin and CD62L.

Results: Marked differences $(p=0,0085)$ were found regarding an increased frequency of PBs in patients with SSC (median=59,3,n=46) as compared to healthy donors(HDs) (median $=48, n=23)$ ). Interestingly, this increase of $\mathrm{PB}$ frequency is accentuated in patients with pulmonary fibrosis(PF) (median $=66,1, \mathrm{n}=21$ ) as compared to patients without PF (median=57,2, $n=22, p=0,08$ ) and HDs (median=48, $n=23$, $p=0,0024)$.The frequency of $P B s$ being positive for the mucosal marker a $4 \beta 7$-Integrin is significantly higher in SSc patients (median $=44,65$, $n=46)$ in comparison with HDs (median $=35,2, n=23)(p=0,0173)$. This increase of $\alpha_{4} \beta 7$-Integrin positive $\mathrm{PB}$ frequency is again more pronounced in patients with PF (median $=43,7, n=21$ ) as compared to patients without PF (median $=30,45, n=22, p=0,1585$ ) and also increased in patients with pulmonary arterial hypertension $(\mathrm{PAH})($ median $=59,9, \mathrm{n}=10)$ in comparison to patients without $\mathrm{PAH}$ (median $=43,6, n=33, \mathrm{p}=0,0829$. Further clinical analyses indicates that $a_{4} \beta 7$-Integrin+lgA+ PBs might possess an increased resistance against $B$ cell depletion therapy with Rituximab (RTX) based on the higher frequency of those cells patients after RTX treatment (median $=65,5, n=3$ ) as compared to patients without RTX treatment (median $=38,55, \mathrm{n}=46, \mathrm{p}=0,0431$ ) and HDs (medi$a n=22,4, n=23, p=0,0085$ ).

Conclusion: The frequency of PBs with a mucosal phenotype is increased in patients with SSc. This increase of mucosal activity is partly associated with pulmonary involvement. PBs with a mucosal phenotype seem to have an increased resistance against $B$ cell depleting therapy. Those findings indicate that mucosal plasmablasts could serve as biomarkers in SSc and will help to develop further therapeutic strategies.

\section{PS032 \\ Regulation von microRNAs durch p63 im Hepatozellulären Karzinom}

Emily Ungermann, Elisabeth Aschenbrenner, Kirstin Pollinger, Claudia Kunst, Martina Müller

Klinik und Poliklinik für Innere Medizin I, Universitätsklinikum Regensburg, Regensburg

Einleitung: Transkriptionsfaktoren der p53-Familie (p53, p63, p73) werden durch zellulären Stress aktiviert und induzieren die Aktivierung verschiedener Zielgene, die zu Zellzyklusarrest und Apoptose führen. In Tumoren wie dem hepatozellulären Karzinom (HCC) können sie sowohl kanzerogene als auch tumorsuppressive Effekte aufweisen. p63 zeichnet sich durch seine Rolle in der Entwicklung von Epithelien und in der Epithelial-Mesenchymalen Transition aus, ein Prozess, der zur Metastasierung von Tumoren führt. microRNAs (miRNAs) sind nicht kodierende RNAs von ca. 22nt Länge, die durch Anlagerung an Ziel-mRNAs diese hemmen und somit zu einer posttranskriptionalen Regulation der Genexpression führen. Die Expression von miRNAs kann wiederum durch Proteine der p53-Familie gesteuert werden. In Vorarbeiten konnten p53-regulierte miRNAs im HCC identifiziert werden. p63 beeinflusst beeinflusst die miRNA-Spiegel im Plattenepithelkarzinom. Über die Rolle von p63 im HCC ist hingegen bislang wenig bekannt. Ziel der Arbeit war daher die Identifizierung von miRNAs, die durch p63 im HCC reguliert werden.

Methoden: $\mathrm{Hep}_{3} \mathrm{~B}$ Zellen wurden mit adenoviralen Vektoren (rAd-p63, rAd-GFP) transfiziert. Nach 24h, 48h und 72h wurden die Zellen geerntet und mittels qPCR die relative Expression von miRNA 34a, miRNA 149, miRNA 192 und miRNA 194 bestimmt. In einem zweiten Schritt wurden transfizierte Zellen in vitro mit HCC-relevanten Therapeutika (Doxorubicin, Bleomycin, Sorafenib, Regorafenib und Tivantinib) behandelt. Die relative miRNA-Expression $24 \mathrm{~h}, 48 \mathrm{~h}$ und $72 \mathrm{~h}$ nach der Behandlung wurde mittels qPCR bestimmt.

Ergebnisse: Die Überexpression von p63 führte nach $24 \mathrm{~h}$ zu einer 1,9-fachen Induktion von miR34a, einer 1,96-fachen Induktion von miR149, einer 3,85-fachen Induktion von miR192 und einer 5,25-fachen Induktion von miR194. Eine in vitro Inkubation von $72 \mathrm{~h}$ mit Doxorubicin führte zu einer Steigerung der p63-abhängigen Expression von miR34a (3,7-fach), miR145 (4,88-fach), miR149 (4,03-fach) und miR192 (2,53-fach). Die Behandlung mit Sorafenib führte zu einer 2,69-fach erhöhten p63-abhängigen Expression von miR192 und einer 2,48-fach erhöhten p63-abhängigen Expression von miR194 nach 72h. Durch Tivantinib wurde nach $72 \mathrm{~h}$ die p63-abhängige Expression von miR192 um das 2,42-fache und die von miR194 um das 3,6-fache verstärkt.

Schlussfolgerung: Wir zeigen erstmals die Induktion der Expression tumorsuppressiver miRNAs durch p63 im HCC. Die beobachteten synergistischen Effekte der p63 Überexpression und HCC-relevanten Therapeutika erweitern zum einen unser Verständnis des komplexen regulatorischen Netzwerks in Tumorzellen und bieten zum anderen potentielle prognostische und therapeutische Ansätze bei der Behandlung des HCC.

\section{PS033}

Die prognostische Bedeutung von Hepcidin bei der chronischen Nierenerkrankung

Johanna Wiedenroth'; Insa Emrich²; Sarah Seiler-Mußler ${ }^{2}$; Kathrin Untersteller'; Gunnar Heine ${ }^{2}$; Danilo Fliser ${ }^{2}$ 
'Universitätsklinikum des Saarlandes und Medizinische Fakultät der Universität des Saarlandes; ${ }^{2}$ Universitätsklinikum des Saarlandes, Homburg

Einleitung: Störungen im Eisenstoffwechsel tragen zur Morbidität und Mortalität von chronisch nierenkranken Patienten bei. Ein Schlüsselhormon des gestörten Eisenstoffwechsels ist Hepcidin, welches die Eisenresorption aus dem Enterozyten inhibiert und daher zum Eisenmangel chronisch nierenkranker Menschen beiträgt. Es wird postuliert, dass bei chronisch nierenkranken Menschen eine chronische Mikroinflammation in einer prognostisch ungünstigen Hochregulation von Hepcidin mündet; allerdings fehlen bislang größere epidemiologische Studien, die diese Hypothese bekräftigen. In der prospektiven CARE FOR HOMe Studie sollte die prognostische Bedeutung erhöhter Plasma-Hepcidinspiegel analysiert werden.

Material \& Methoden: Seit 2008 wurden insgesamt 584 chronisch nierenkranke Patienten der Stadien KDIGO G2 bis $\mathrm{G}_{4}$ in die fortlaufende CARE FOR HOMe Studie eingeschlossen. Bei 555 Teilnehmern erfolgten Messungen von Hepcidin und von konventionellen Parametern des Eisenstoffwechsels aus zum Zeitpunkt des Einschlusses asserierten Proben. Alle Teilnehmer wurden mittels standardisierter Follow-Up Visits jährlich nachverfolgt. Als kombinierter Endpunkt der CARE FOR HOMe Studie war das Auftreten von atherosklerotischen Ereignissen und Todesfälle vordefiniert. Sekundäre Endpunkte waren die Einzelkomponenten dieses kombinierten Endpunkts.

Ergebnis: Im Nachbeobachtungszeitraum von $5.1 \pm 2.1$ Jahren erreichten 157 Patienten den vordefinierten kombinierten kardiovaskulären Endpunkt. 123 Patienten erlitten ein kardiovaskuläres Ereignis, und 98 Patienten verstarben. Erwartungsgetreu korrelierte Hepcidin eng mit Ferritin ( $r=0.709 ; p \leq 0.001)$, jedoch nur schwach mit CRP ( $r=0.106 ; p=0.013)$ und eGFR ( $r=-0.192 ; p<0.001)$. Überraschenderweise zeigte sich in univarianten Kaplan Meier Analysen nach Stratifizierung in Tertile kein signifikanter Zusammenhang zwischen Hepcidin und den vordefinierten Endpunkten (primärer Endpunkt $p=0,381$; sekundäre Endpunkte a) kardiovaskuläre Ereignisse $p=0,167 ; b$ ) Todesfälle jedweder Genese $p=0,606$ ).

Zusammenfassung: Trotz der tierexperimentell postulierten zentralen pathophysiologischen Bedeutung von Hepcidin für die Komorbidität chronisch nierenkranker Menschen erwies sich Hepcidin in der CARE FOR HOMe Studie nicht als ungünstiger Prognosemarker. Diese Beobachtung kann klinisch bedeutsam werden, da Hepcidin als Target für neue pharmakologische Interventionen in den Eisenhaushalt untersucht wird.

\section{PS151}

Lebensstiländerung in Patienten mit nicht alkoholischer Fettlebererkrankung (NAFLD) durch eine Internet-betreute Sportintervention - Die HELP Studie

\section{Daniel Pfirrmann'; Yvonne Huber ; Jörn M. Schattenberg ${ }^{2}$; Perikles Simon \\ ${ }^{1}$ Institut für Sportwissenschaften, Mainz; ${ }^{2}$ Universitätsmedizin Mainz, Mainz}

Problemstellung: Eine Änderung des Lebensstils zur Verbesserung des metabolischen Risikoprofils wird zur Prävention und Therapie der nicht-alkoholischen Fettleber (NAFLD) empfohlen. Ziel ist es, den Kalorienbedarf an den Kalorienverbrauch durch aktive Gestaltung des Alltags anzupassen. Die Empfehlungen für körperliche Aktivität bei NAFLD sind eine Kombination aus 150 Minuten moderatem Ausdauertraining und zweimal wöchentlich Krafttraining. Berichtete Barrieren in der Umsetzung sind oft unzureichendes Wissen, zeitliche Probleme und auch fehlendes Interesse. Die Bedeutung von supervidierten Pro- grammen für die Adhärenz an Empfehlungen zur Lebensstiländerung im Kontext von Lebererkrankungen ist unzureichend untersucht. Zusätzlich limitieren hoher personeller und finanzieller Aufwand die individuelle Betreuung. Deshalb wurde die HELP Studie, die mit einer internetbasierten, individuellen Trainingsbetreuung von Patiente mit NAFLD arbeitet, durchgeführt.

Methode: Die HELP Studie ist eine prospektive, einarmige Studie (ClinicalTrials.gov Identifier: NCT 02526732) zur Untersuchung der Adhärenz von Lebensstiländerungen in Patienten mit histologisch gesicherter NAFLD. In einem Zeitraum von 24 Monaten wurden 44 Patienten eingeschlossen. Nach einer internistischen und sportmedizinischen Untersuchung erhielten die Studienteilnehmer über acht Wochen ein individualisiertes Kraft- und Ausdauerprogramm. Die Betreuung der Patienten erfolgte über eine eigens dafür entwickelte web-basierte Plattform. Durch regelmäßige Rückmeldung zum Trainingsprotokoll, adaptierte der Sporttherapeut den Trainingsplan an die individuellen Bedürfnisse und Probleme der Patienten wöchentlich.

Ergebnisse: 42 von den 44 eingeschlossenen Patienten haben zum Analysezeitpunkt das achtwöchige Trainingsprogramm komplett durchlaufen. Zum Studienende zeigte sich ein signifikanter Anstieg in der wöchentlichen körperlichen Aktivität (P

\section{Altern und Altersmedizin I}

\section{PS036}

Stellenwert der B-Mode-Sonographie in der Diagnostik muskulärer Aspekte im akutgeriatrischen Setting

Heinrich Burkhardt; Laura Parigger

Universitätsmedizin Mannheim, Mannheim

Hintergrund: Die Diagnostik muskulärer Aspekte, um sowohl das Ausmaß der Sarkopenie bzw. auch Gesichtspunkte des Deconditioning im akutgeriatrischen Bereiches zu erfassen ist zunehmend von Interesse. Bisher ist nicht ausreichend geklärt, ob in diesem Zusammenhang auch direkte Maße relevanter Muskulatur durch B-Mode-Sonographie erfasst werden können.

Methoden: 84 Patienten über 65 Jahre, die aufgrund diverser klinischer Probleme in einer akutgeriatrischen Einheit behandelt werden mussten, wurden in die Untersuchung mit einbezogen. Es wurden in einem standardisierten Verfahren definierte anatomische Diameter der Mm. quadrizeps femoris und gastrocnemius mittels B-Mode-Sonographie in einem bed-side Ansatz erfasst. Zur Bestimmung der Reliabilität wurden 3 aufeinanderfolgende Ultraschall-Messungen durchgeführt. Zugleich wurde die Kraft der Muskulatur mit einem ebenfalls bettseitig einsetzbarem Dynamometer gemessen sowie mittels BIA der Skelettmuskel-Index bestimmt und gemeinsam mit der Handkraft zu Abschätzung der Validität herangezogen.

Ergebnisse: 44 Frauen und 40 Männer, Alter 65-96 Jahre (78) wurden untersucht. 34 nahmen an einem frührehabilitativen Programm teil. Der ADL-Wert betrug 0-100 (85). Vollständige Ultraschall-Messreihen konnten für 80 Patienten gewonnen werden (4 Patienten hatten die US-Untersuchung abgelehnt). Für die Übereinstimmung der jeweils drei Messungen wurde für den M. quadrizeps ein Cronbach alpha von 0.99 , für den M. gastrocnemius 0.98 gefunden. Weiter fanden sich positive Korrelationen (0,29 bis 0.49) zwischen den Muskeldiametern, Handkraft und dem errechneten Skelettmuskel-Index. 
Diskussion: Die B-Mode-Sonographie zeigt eine ausreichende Praktikabilität und Reliabilität, um auch in einem bed-side-Ansatz im akutgeriatrischen Setting angewendet zu werden. Die gewonnenen Diameter zeigten ferner den erwarteten Bezug zu anderen Aspekten der Muskel-Performance.

\section{PS037}

Repräsentanz geriatrischer Aspekte in deutschen und US-amerikanischen Leitlinien

Heinrich Burkhardt; Franziska Scherr

Universitätsmedizin Mannheim, Mannheim

Hintergrund: Leitlinien sind unverzichtbarer Bestandteil der modernen Medizin geworden, werden aber immer wieder wegen mangelnder Berücksichtigung geriatrischer Aspekte kritisiert.

Methoden: Nach einer im Vorfeld festgelegten Suchstrategie wurden Leitlinien aus Deutschland (AWMF) und USA (National Guideline Clearing House) anhand deren Titel ausgewählt. Die Themenfelder waren relativ weit gefasst, sollten aber geriatrische Aspekte, entweder aufgrund der Art des behandelten Gesundheitsproblems oder der erhöhten Prävalenz im Alter erwarten lassen (z.B. Diabetes, Schlaganfall, Synkope, Demenz, Urininkontinenz). Es wurde versucht jeweils korrespondierende Leitlinien aus Deutschland und den USA zu betrachten. Die Leitlinien wurden sodann nach einem zuvor festgelegten Schema auf die etwaige Berücksichtigung geriatrischer Aspekte (z.B. Kommentare zu geriatrischen Syndromen oder Risiken bzw. auch Multimorbidität) hin analysiert und dies in einem Score erfasst und quantifiziert (o-28). 5 inhaltliche Themenfeeder wurden strukturiert bewertet: Ältere Patienten allgemein, Kognition, Lokomotion, Polypharmazie, Multimorbidität.

Ergebnisse: Es wurden 30 Leitlinien analysiert (13 deutsche, 17 US-amerikanische). Die höchste Kategorie ( $75 \%$ und mehr der erreichbaren Punkte) erreichte nur die US-amerikanische Diabetes-Leitlinie der ADA. In die Kategorie II wurden 13 Leitlinien bewertet (50-75\% der erreichbaren Punkte), 11 weitere in Kategorie III (25-50\% der erreichbaren Punkte) und 5 Leitlinien in die niedrigste Kategorie (unter $25 \%$ ). Von den 5 analysierten inhaltlichen Themenfelder fand sich das Thema Polypharmazie am wenigsten repräsentiert (in 41,4\% der Leitlinien unter $25 \%$ der erreichbaren Punkte.

Diskussion: Es ergibt sich ein recht heterogenes Bild der aktuellen Repräsentanz geriatrischer Themen in den Leitlinien. Insgesamt erreichen nur wenige Leitlinien eine zufriedenstellende Berücksichtigung geriatrischer Aspekte, wobei das Thema der Polypharmazie besonders betroffen ist. Andererseits sind in den meisten Leitlinien zumindest teilweise einige Aspekte berücksichtigt. Es bedarf eines strukturierten Auftrages bei Erstellung der Leitlinien, die geriatrischen Besonderheiten entsprechend abzubilden oder zu kommentieren.

\section{PS038 \\ Pleuraerguss als Erstmanifestation des Nierenzellkarzinoms- Fallbericht}

Ioana Duca'; Romeo loan Chira ${ }^{2}$; Cristina Sabo²; Daniela Fodor'; Ionela Miron'; Dan Lucian Dumitrascu'

\section{Med.Klinik Klausenburg; ${ }^{2} 1$. Med.Klinik Klausenburg}

Ziel: Lokalisierte Pleurametastasen des Nierenkarzinoms sind selten anzutreffen. Nekrotische Anteile verschiedener Raumforderungen stellen oft eine differentialdiagnostische Herausforderung dar.
Material und Methoden: Wir berichten über den Fall einer 59.jährigen Patientin, Raucherin, bekannt mit Hypertonie, welche nach mehreren ambulanten Antibiotikatherapiekuren bei V.a.Pneumonie, wegen Dyspnoe, Reizhusten, rechte Thoraxschmerzen und Gewichtsverlust eingewiesen wurde. Es wurden folgende Untersuchungen durchgeführt: klinischer Befund, Laborwerte, Sonographie, Röntgenthorax, CT und Histologie.

Ergebnisse: Die klinische Untersuchung stellte einen schlechten Allgemeinzustand, links-lateralen Decubitus, Tahypnoe, reduziertes vesikuläres Atemgeräusch und Dumpfheit der unteren Hälfte der rechte Lunge, Dehydrierung, perorale Zyanose, harte $5 \mathrm{~cm}$ grosse Raumforderung (RF) rechts präaurikulär, diffuse Abdomenschmerzen bei Palpation und erhöhten therapieresistenten Blutdruck (190/130mm $\mathrm{Hg}$ ), ohne Fieber, fest. Der Laborbefund zeigte Leukozytose, leicht erhöhtes Kreatinin, erhöhte CRP und alkaline Phosphatase, Hypercholesterolemie, Hypertriglyceridemie, mikroskopische Hämaturie, Proteinurie und negative Urokultur. Röntgen-Thorax schilderte nur den grossen rechten Pleuraerguss, während zusätzlich natives Thorax-CT mehrere Lungenherde und mediastinale Lymphknotenvergrösserungen aufwies. Pleurapunktion erwiderte ein hämorrhagisches Exudat mit malignen Zellen. Abdomensonographie wies eine $6.2 / 5 \mathrm{~cm}$ grosse inhomogene linke NierenRF mit zystischen Anteilen und echoreichem Zentrum, und punktförmigen Dopplersignal auf; die linke $\mathrm{Ne}$ benniere wies eine $5 \mathrm{~cm}$ grosse RF auf. Thoraxultraschall wies mehrere echoreichen unregelmässige RF der Pleura mit V.a. Metastasen, regionale Verdickung der parietalen Pleura und subpleurale LungenRF auf. Ebenso inhomogen erwies sich die rechte präaurikulaere RF der Ohrspeicheldrüse $(4 \times 3 \mathrm{~cm})$, mit unregelmässiger Abgrenzung und $20 \%$ zystischem Anteil mit mässiger Vaskularisation, mit V.a.Primärtumor mit nekrotischen Anteilen.

Enddianose wurde mittels Biopsie gestellt: Nierenzellkarzinom mit klaren Zellen, während die Histologie den Parotistumor als pleomorphes Adenom einordnete.

Schlussfolgerung: Besonderheiten des Falles stellten die späte Diagnosestellung des Nierenzellkarzinoms im Stadium der Pleura- und Lungenmetastasen, sowie die synkronen nekrotisch enthaltenen Tumoren der Niere, Nebenniere, und Ohrspeicheldrüse dar.

\section{PS039 \\ Sarcopenia and Physical fRailty IN older people: multi-compo- nenT Treatment strategies: Design und Methode der EU-Studie SPRINTT}

Ellen Freiberger; Daniel Schöne; Anna Franke; Susann Gotthardt; Veronika Reith; Hanna Rempe; Gabrielle Sieber; Cornel C. Sieber

\section{Friedrich-Alexander-Universität Erlangen-Nürnberg}

Einleitung: Die Syndrome (körperliche) Gebrechlichkeit („Frailty“) und alternsbezogener Verlust der Muskelmasse ("Sarkopenie“) wurden in den letzten Jahren aufgrund ihrer hohen Relevanz bezüglich Mobilität, Selbständigkeit und Lebensqualität bei älteren Menschen vermehrt zum Untersuchungsgegenstand. Multimodale Interventionen mit dem Fokus auf strukturierter Bewegung kombiniert mit optimierter Ernährung zeigten sich als effektive Komponenten im Management dieser geriatrischen Syndrome. Allerdings sind multimorbide, funktionell eingeschränkte ältere Menschen mit Sarkopenie in großen Interventionsstudien unterrepräsentiert. Deshalb fördert die EU gemeinsam mit den Industriepartnern (IMI) die Phase III multizentrische, einfach-verblindete, randomisierte und kontrollierte Studie SPRINTT. Das Institut für Biomedizin des Alterns (IBA) an der FAU ist der deutsche Partner weiterer europäischen Zentren. 
Ziel: Ziel ist es, Machbarkeit und Effekt eines spezifisch für körperlich gebrechliche, sarkopene ältere Menschen entwickeltes Trainingsprogramm zu evaluieren.

Methode: Methode: Im Zeitraum von April 2015 bis Oktober 2017 durchliefen am IBA 1285 potentiell geeignete Personen ein Screening. Davon konnten 123 Teilnehmer (9,6\%) in die Studie randomisiert werden. Haupteinschlusskriterien waren neben einem Alter von 70 Jahren und älter sowie selbständig lebend i) die Fähigkeit 400 Meter in $<15$ min zu gehen, ii) funktionelle Einschränkungen (Short Physical Performance Battery (SPPB) 3-7 (80\%), 8-9 (20\%) und iii) eine geringe DXA-gemessene appendikuläre Skelettmuskelmasse (Total: ơ $<19,75 \mathrm{~kg}$, $\subsetneq<15,02$ kg; BMl-adjustiert: $९<0,789$, $\subsetneq<0,512)$. Die der Interventionsgruppe zugeteilten Teilnehmer $(n=62)$ absolvieren ein, in der Intensität moderat-intensiv, strukturiertes Training mit mehreren Komponenten (Ausdauer, Kraft, Gleichgewicht: 2x90 Minuten/ Woche unter Supervision \& $3 \times 30$ Minuten/Woche Heimtraining). Zusätzlich erhalten sie mindestens $1 x / J a h r$ eine individualisierte Beratung zu Ernährung und körperlicher Aktivität. Die Teilnehmer der Kontrollgruppe $(n=61)$ nehmen, im Sinne der Hilfe zur Selbsthilfe, 2X/ Monat an einem Schulungsprogramm teil (6o Minuten Vorträge über Gesundheit und Krankheit, Übungen für Gedächtnistraining und jeweils 10 Minuten Stretching der oberen Extremitäten). Die Teilnehmer beider Gruppen folgen dem Programm bis zu 36 Monaten und werden im Zeitabstand von 6 Monaten nachuntersucht. Primärer Ergebnisparameter ist die Mobilisation, operationalisiert durch den 400 Meter-Gehtest.

Ausblick: Durch SPRINTT wird ein Trainingsprogramm angeboten um Mobilität, Funktion und damit Selbständigkeit bei körperlich gebrechlichen und sarkopenen, älteren Menschen zu erhalten. Des Weiteren sollen am IBA laufende Substudien zu SPRINTT mit den Themen Sarkopeniescreening, Gangveränderung, Sturzprädiktion und Ernährungseinstellung zusätzliche wichtige Daten generieren.

\section{PS040 \\ Der Geriatrie Check der Baden-Württembergischen Kranken- hausgesellschaft als Instrument zur Identifikation geriatrischer Patienten in der Notaufnahme}

Tobias Gerhard'; Kristina Mayer'; Ulrike Braisch²; Jochen Klaus³; Dhayana Dallmeier'; Michael D. Denkinger ${ }^{4}$

'Agaplesion Bethesda Klinik Ulm / Geriatrisches Zentrum Ulm/AlbDonau; ${ }^{2}$ Universität Ulm; ${ }^{3}$ Universitätsklinikum Ulm; ${ }^{4}$ Agaplesion Bethesda Klinik Ulm

Hintergrund: Die Identifikation geriatrischer Patienten wird als der Schlüssel zur patienten- und bedarfsgerechten Versorgung anerkannt. Mit Hilfe des in Baden Württemberg im Rahmen des Geriatriekonzepts entwickelten Geriatrie-Checks soll sowohl die Identifikation eines geriatrischen Patienten in der Notaufnahme als auch die Entscheidung hinsichtlich eines daraus resultierenden Behandlungsbedarfs gelingen. Ziel war daher die Evaluierung dieses Instruments bezüglich Diskriminierung und Aussagekraft.

Methoden: Im Rahmen einer prospektiven Kohortenstudie wurden Patienten >70 Jahre, die in der internistischen Notaufnahme des Universitätsklinikums Ulm zwischen November 2015 und April 2016 behandelt wurden, eingeschlossen. Barthel-Index, Wohnsituation, Pflegestufe und andere Parameter wurden bei Aufnahme und nach drei Monaten ermittelt. Wir berechneten Sensitivität, Spezifität, die negative und positive prädiktive Werte (NPW und PPW) mit dem bisher in der Notaufnahme verwendeten "Identification of Seniors At Risk" (ISAR) als Goldstandard.
Ergebnisse: Insgesamt wurden 146 Patienten (Alter median 79,8 Jahre (Q1 77,0; Q3 86,7), Männer 49,3\%) eingeschlossen. Davon wurden 107 $(73,3 \%)$ Patienten als geriatrisch laut Geriatrie-Check identifiziert. Verglichen zu nicht-geriatrischen Patienten waren geriatrische Patienten im Durschnitt älter (median Alter 81,0 vs. 77,4 Jahre), hatten bei Aufnahme mehr Dauermedikamente (7,0 vs. 5,0) ein, hatten einen niedrigeren Barthel-Index (95 vs. 100), sowie einen erhöhten Informant Questionnaire on cognitive decline (IQCode) Score vor Aufnahme (3,3 vs. 3,1). Ebenso bestand ein erhöhtes Risiko für Malnutrition (Nutritional Risk Screening Score 76 vs. 20), und Delir (Kurzer Mentaler Test Score 70 vs. 18). Bei Aufnahme waren $14,0 \%, 9,3 \%$ und 2,8\% der als geriatrisch identifizierten Patienten jeweils in Pflegestufe 1, 2 und 3. Der Geriatrie-Check zeigte eine Sensitivität vom 82,0\% [95\% Konfidenzintervall (KI) 73,$9 ; 88,5]$ und eine Spezifizität vom 62,1\% [95\% KI 42,3; 79,3] verglichen zum ISAR. Der PPW war $89,7 \%$, mit einem NPW von $46,1 \%$. Nach drei Monaten beobachtete man eine signifikante Verschlechterung des Barthel-Indexes der geriatrisch eingestuften Patienten von 95 (Q1 75; Q3 100) bei Aufnahme zu 80 (Q1 45; Q3 100). Zusätzlich stieg hier der Anteil der im Pflegeheim wohnenden Patienten von 10,3\% auf 21,2\%, mit einer assoziierten signifikanten Verschlechterung der Pflegestufe verglichen zu nicht-geriatrischen Patienten.

Diskussion: Die als "geriatrisch“ identifizierten Patienten unterscheiden sich in mehreren Punkten von den "nicht geriatrischen“. Verglichen zu ISAR zeigt der Geriatrie-Check eine gute Sensitivität und einen guten PPW, bei weniger optimalem NPW. Zusätzlich konnten "geriatrische" Patienten durch eine Verschlechterung des Barthel-Indexes und der Pflegestufe nach drei Monaten als Risikogruppe identifiziert werden. Weitere Untersuchungen zur Evaluierung dieses Instruments mit größeren Kohorten sind notwendig.

\section{PS040A \\ Selbstmanagement und Schulungserfahrung inhalativer Pharmaka bei älteren COPD-Patienten}

Heinrich Burkhardt; Phillip Engert

Universitätsmedizin Mannheim, Mannheim

Hintergrund: Die Anwendung inhalativer Pharmaka ei der COPD ist komplex und die Anzahl der möglichen Fehlerquellen groß ist. Ziel dieser Arbeit war es, das Selbstmanagement älterer COPD-Patienten bez. inhalativer Pharmaka zu analysieren und mögliche Barrieren zu identifizieren

Patienten und Methoden: 49 stationär behandelte Patienten über 65 Jahre wurden bezüglich Ihrer Krankheitsanamnese, Alltagskompetenzen, Lungenfunktion und Körperkomposition untersucht und befragt. Die Handhabung inhalativer Pharmaka wurde anhand eines eigens dafür entworfenen Scores bewertet.

Ergebnisse: $59,2 \%$ der Patienten zeigten große Unsicherheiten bei der Applikation dieser Medikamente. Als ein wesentlicher Einflussfaktor konnten Patientenschulungen identifiziert werden: Ungeschulte Patienten (25\%) beherrschten die Handhabung signifikant schlechter (U-Test $p=0,000)$ und kein ungeschulter Patient erreichte ein zufriedenstellendes Ergebnis. Patienten profitierten außerdem von einer längeren Schulungsdauer (Spearman Rho 0,$536 ; p=0,000$ ) und mehrfach durchgeführten Schulung (Spearman Rho 0,529; $p=0,000$ ). Weiterhin konnten Zusammenhänge zwischen kognitiven und motorischen Fähigkeiten und dem Selbstmanagement gezeigt werden: MMSE: Spearman Rho -0,497; $\mathrm{p}=0,000$; Geldzähl-Test: Spearman Rho $0,332 ; p=0,014)$. Dass das Selbstmanagement möglicherweise im Rahmen eines geriatrischen Gebrechlichkeitssyndroms durch abnehmende Kraft eingeschränkt ist, konnte in dieser Stichprobe aber nicht eindeutig gezeigt werden: Dies galt sowohl für die Handmuskelkraft ( 
Spearman Rho -0,117; $\mathrm{p}=0,400$ ) wie für Surrogatmarker der Atemmuskelkraft (Po,1 Rho -0,222; $p=0,107$; Pmax Rho 0,156; $p=0,259$ ). Lediglich der mittels Body-Impedanzanalyse abgeleitete Skelettmuskelmassenindex korrelierte hier signifikant (Rho-0,420; $\mathrm{p}=0,002)$.

Schlussfolgerung: Unsicherheiten in der Handhabung der inhalativen Medikamente sind bei älteren COPD-Patienten sehr häufig und beruhen teilweise auf mangelnder Schulung, welches besserbar wäre. Andererseits spielen auch funktionelle Einschränkungen eine Rolle (Kognition), wohingegen keine starke Assoziation zwischen der Muskelkraft und dem Selbstmanagement aufgezeigt werden konnte.

\section{PS041}

Fehlender Einfluss der Skelettmuskelmasse auf die Bestimmung des Sturzrisikos selbständig lebender Senioren mit funktionellen Einschränkungen

Susann Gotthardt'; Anna Franke'; Tina Auerswald '; Carolin Kraska'; Marina Mellou'; Anne Hofmann'; Veronika Reith'; Ellen Freiberger'; Cornel C. Sieber ${ }^{1}{ }^{2}$; Daniel Schoene

${ }^{1}$ Friedrich-Alexander-Universität Erlangen-Nürnberg; ${ }^{2}$ FriedrichAlexander-Universität Erlangen-Nürnberg (FAU), Barmherzige Brüder Krankenhaus Regensburg

Einführung: Die Abnahme der Muskelmasse ist ein bekanntes Phänomen des Alterns, welches u.a. assoziiert ist mit einer reduzierten körperlichen Leistungsfähigkeit, z.B. Ganggeschwindigkeit, Gleichgewicht und Kraft, wichtigen Risikofaktoren für Stürze im Alter. Der Einfluss der Muskelmasse auf das Sturzrisiko funktionell eingeschränkter alter Personen, einer besonders vulnerablen Gruppe, ist bisher nicht ausreichend untersucht worden.

Methodik: Die im Rahmen des Screeningprozesses (bis Juli 2017) einer randomisierten Kontrollstudie (SPRINTT-Studie, www.mysprintt.eu) durchgeführten Messungen des beteiligten deutschen Testzentrums wurden zur Beantwortung der Frage verwendet. Teilnehmer waren i) selbständig lebende, ii) funktionell eingeschränkte (Short Physical Performance Battery (SPPB) 3-9) Menschen iii) $\geq 70$ Jahre iv) ohne starke kognitive Beeinträchtigungen (Mini-Mental-Status-Test>23). Der SPPB ist eine funktionelle Testbatterie und beinhaltet Messungen von Gleichgewicht (geschlossener Stand, Semitandem, Romberg), habitueller Ganggeschwindigkeit $(4 \mathrm{~m})$ und Kraft (5-maliges schnelles Aufstehen) mit Kategorienpunktwerten von o-4 und einem Gesamtpunktwert zwischen 0-12 (geringere Werte $=$ schlechtere Funktionalität). Ganzkörperscans per Dual-Röntgen-Absorptiometrie (DXA) wurden durchgeführt zur Bestimmung der appendikulären Magermasse (Rohwert und BMI-adjustiert) als Parameter der Skelettmuskelmasse. Als Stürzer wurden Personen klassifiziert, welche mindestens 2 Stürze in den vergangenen 12 Monaten berichteten. Die Analysen wurden adjustiert für Geschlecht.

Ergebnisse: Dreihundert-neunzehn Personen (Durchschnittsalter $80 \pm 5$ Jahre, $77 \%$ weiblich) hatten Messwerte für alle relevanten Variablen und von diesen stürzten $76(24 \%)$ mehrfach. Stürzer und Nicht-Stürzer unterschieden sich nicht in ihrer Muskelmasse, weder bei den Rohwerten $\left(F_{316,1}=0,556, p=0,456\right)$ noch bei den BMl-adjustierten Werten $(F 316,1=0,104, p=0,747)$. Einen kleinen klinisch nicht relevanten Zwischengruppenunterschied zeigte der SPPB Gesamtpunktwert (Nicht-Stürzer 7,2 $\pm 1,4$ vs Stürzer 6,9 $\pm 1,6, F 316,1=3,919$, $p=0,049$; beide Gruppen Median $=7$ ). Von den SPPB Kategorien war lediglich die Gleichgewichtsfähigkeit mit dem Sturzrisiko assoziiert, als das ein besseres Gleichgewicht ein protektiver Faktor war (OR=0,95 (0,90$0,99), p=0,022$ ). Bei einer Klassifizierung in frail (SPPB

\section{Altern und Altersmedizin II}

\section{PS042}

Gangvariabilität einer älteren, sarkopenen Risikopopulation in Abhängigkeit von verschiedenen Gangbedingungen

Sebastian Krumpoch; Veronika Reith; Christian Meyer; Cornel C. Sieber; Ellen Freiberger

\section{Friedrich-Alexander-Universität Erlangen-Nürnberg}

Hintergrund: Ältere Menschen mit klinisch auffälligem Gangbild haben häufiger Sturzereignisse und ein höheres Sterblichkeitsrisiko. Mit dem Alter einhergehende kognitive, somatosensorische und funktionelle Einschränkungen führen zu größerer Variabilität quantitativer und qualitativer Gangparameter. Altersbedingte Sarkopenie und die abnehmende, subkortikale Kontrolle des Gangs müssen mit einem größeren Einsatz von Aufmerksamkeitsressourcen kompensiert werden. Das Dual-Task Paradigma ist die Referenzmethode für die Überprüfung und Evaluation von Automatisierungsgraden und Interferenz zweier, gleichzeitiger Prozesse [1]. Der Einfluss von Sarkopenie auf das Gangbild unter Single-Task Bedingung und Dual-Task Bedingung wurde bisher nicht ausreichend erforscht.

Ziel: Im Zuge dieser Substudie vom SPRINTT Interventionsprojekt (siehe Einführungsposter) werden deskriptiv die Veränderungen quantitativer und qualitativer Gangparameter mit ihrer Variabilität unter verschiedenen Gangbedingungen in einer selbständig lebenden, sarkopenen Risikopopulation untersucht.

Methode: Im Zuge dieser Substudie vom SPRINTT Interventionsprojekt werden 60 ältere Teilnehmer mit Prefrailty-Syndrom rekrutiert. 40 Teilnehmer erfüllen die funktionellen und muskulären Sarkopenie Kriterien und 20 weitere Teilnehmer erfüllen die funktionellen Parameter der Sarkopenie, haben aber eine zu hohe Muskelmasse. Die Muskelmasse der Probanden wird mit Hilfe eines Ganzkörper-DXA -Scans gemessen. Die Teilnehmer gehen über ein elektronisches, klinisch validiertes Ganganalysesystem (GAITRite). Der GAITRite-Teppich erfasst über Druckrezeptoren sämtliche räumliche und zeitliche Gangparameter. Insgesamt 8-mal wird das Gangbild jedes Teilnehmers gemessen und dabei kommen Single-Task und Dual-Task Bedingungen zum Einsatz. In den Single-Task Bedingungen müssen die Probanden jeweils 3-mal entweder mit normaler oder schneller Geschwindigkeit über den GAITRite-Teppich gehen. In den Dual-Task Bedingungen wird den Teilnehmern insgesamt 2-mal eine kognitiv anspruchsvolle Aufgabe gestellt, während sie über den Ganganalyseteppich gehen. In allen Gangsituationen werden wichtige quantitative Gangparameter, wie z.B. "Ganggeschwindigkeit" und "Schrittlänge" sowie qualitative Gangparameter wie z.B. der Einbeinstand" oder die "Schrittzeit" erfasst. Auch die Variabilität dieser Gangparameter wird untersucht.

Ausblick: Der Beitrag stellt die Ergebnisse von 60 selbständig lebenden Teilnehmern im Alter von 70 Jahren und älter in den qualitativen und quantitativen Ganganalysen und ihre Variabilität dar. 1.Hausdorff, J.M., et al., Dual-Task Decrements in Gait: Contributing Factors Among Healthy Older Adults. The Journals of Gerontology Series A: Biological Sciences and Medical Sciences, 2008. 63(12): p. 1335-1343. 


\section{PS043}

Multidimensionale Prognoseberechnung bei älteren multimorbiden Patienten auf nephrologischer Akutstation: Baseline \& Follow-Up-Ergebnisse der MPI_InGAH Studie

Anna Maria Meyer'; Ingrid Becker²; Paul Brinkkötter²; Alberto Pilotto $^{3}$; Thomas Benzing ${ }^{2}$; Maria Cristina Polidori ${ }^{2}$

${ }^{1}$ Klinik II Innere Medizin, Uniklinik Köln; ${ }^{2}$ Uniklinik Köln; ${ }^{3}$ Ente Ospedaliero Ospedali Galliera

Hintergrund und Ziel: Die klinische Entscheidungsfindung ist bei älteren multimorbiden Patienten ein komplexer Prozess, bei dem die Prognose eine entscheidende Rolle spielt. Eine multidimensionale Prognoseberechnung, basierend auf einem umfassenden geriatrischen Assessment (Comprehensive Geriatric Assessment, CGA), ist durch die Erhebung des Multidimensionalen Prognostischen Indexes (MPI) möglich. Ziel dieser prospektiven Beobachtungsstudie ist eine CGA-MPI-basierte Prognoseberechnung älterer multimorbider Patienten schon auf der Akutstation durchzuführen, um früh Faktoren zu erfassen, die die klinische Entscheidungsfindung auf Station und den Krankheitsverlauf nach der Entlassung beeinflussen.

Methoden: Es wurden 200 multimorbide Patienten (70+Jahre) auf einer nephrologischen Akutstation rekrutiert. Der MPI wird bei Aufnahme und Entlassung erhoben und berechnet, dabei werden medizinische, psychosoziale und funktionelle Aspekte, sowie Geriatrische Syndrome und Ressourcen systematisch erfasst. Der MPI beinhaltet Gesundheitszustand (CIRS), Alltagsfunktionen (ADL, IADL), Ernährungszustand (MNA), Kognition (SPMSQ), Dekubitusrisiko (ESS) sowie sozialen Status und Medikamentenanzahl.

Ergebnisse: Das Durchschnittsalter der Patienten beträgt 78,3 Jahren, die mediane Verweildauer liegt bei 8 Tagen. Bei Aufnahme wurde für die Mehrzahl der Patienten (73,3\%) ein mittlerer MPI errechnet, bei $17,8 \%$ der Patienten war der MPI hoch, bei 8,9\% war der MPI niedrig. Der mediane MPI bei Aufnahme auf Station ist 0,52. Bei der Verlaufsbeobachtung nach 3 Monaten beträgt die Mortalität $16 \%$, mehr als die Hälfte der Patienten (54,5\%) wurden rehospitalisiert.

Es konnte ein signifikanter Zusammenhang zwischen Hospitalisierungsdauer (Length Of Stay, LOS) und der Inanspruchnahme von ambulanter Pflege und Rehospitalisierung erkannt werden. Ein signifikanter Zusammenhang konnte ebenfalls zwischen MPI bei Aufnahme und Mortalität, Pflegegrad, Inanspruchnahme von Pflegedienst, Rehospitalisierung und Medikamentenzahl gezeigt werden. Signifikant mit einer steigenden Anzahl an Geriatrischen Syndromen sind die Medikamentenanzahl, Pflegegrad und die Inanspruchnahme ambulanter Pflege assoziiert. Mit einer steigenden Anzahl an Geriatrischen Ressourcen sind signifikant negativ korreliert die Medikamentenanzahl, Pflegegrad, Inanspruchnahme ambulanter Pflege und Institutionalisierung.

Zusammenfassung: Mit der Durchführung des CGA mit MPI auf Akutstation können einige Faktoren erhoben werden, die den Krankheitsverlauf nach 3 Monaten vorhersagen können. Besonders von Bedeutung für den Krankheitsverlauf scheinen der LOS, der MPI, die Anzahl Geriatrischer Syndrome \& Ressourcen zu sein, die einen großen Einfluss auf Krankheit, Erholung und Mortalitätsrisiko haben und somit die klinische Entscheidungsfindung auf Station beeinflussen sollten. Weitere Forschung ist notwendig um das Feld der klinischen Entscheidungsfindung zu optimieren und um die Ressourcen der älteren Patienten aufzudecken und zu nutzen.
PS044

QMCI-G - 3-5 Minutes To Screen For Dementia And Mild Cognitive Impairment In Germany 1 - A Pilot Study

Thomas Mross'; William David Molloy ${ }^{1}$; Rónán O'Caoimh²; Sheila Mary McSweeney ${ }^{3}$

${ }^{1}$ Mercy University Hospital Cork; ${ }^{2}$ University Hospital Galway; ${ }^{3}$ St John's Institute of Dermatology London

Background: The Quick Mild Cognitive Impairment (QMCl) screen is a rapid assessment tool for the identification of mild cognitive impairment $(\mathrm{MCl})$ and dementia. It is also capable of assessing normal physiological cognitive decline across different age groups. While the Mini-Mental State Examination and Montreal Cognitive Assessment are used worldwide in the assessment of cognitive decline, the QMCI screen has shown superior sensitivity and specificity in several studies in English-speaking countries and its use is growing. Therefore we have decided to translate and begin validation of the $\mathrm{QMCl}(\mathrm{QMCl}-\mathrm{G})$ for the benefit of German-speaking countries.

Aim: To prepare a German translation of the $\mathrm{QMCl}$ screen and pilot its use in a cohort of patients at risk of $\mathrm{MCl}$ and dementia in preparation for the "QMCl-G - 3-5 Minutes To Screen For Dementia And Mild Cognitive Impairment In Germany 2 - Validation" study.

Methods: The QMCI was translated by a native German speaker along with the guide booklet which describes how to administer the test. Several assistants in an Internal Medicine Specialist Centre were trained on how to administer the test. Over four weeks patients were referred randomly to take the QMCI-G screen. Consent was obtained. The inclusion criteria specified an age of at least 70 years or older. All patients tested below that age had expressed concern about their cognition in person or via collateral from their next of kin. Total scores were out of 100 and cutoffs for normal cognition, $\mathrm{MCl}$ and dementia from the English QMCI were used.

Results: Overall 57 patients were tested, including 22 men (38.6\%) and 35 women $(61.4 \%)$. Twelve patients $(21.1 \%)$ had greater than 12 years of education, 57 (78.9\%) less than 12 years. We found 38 patients $(66.7 \%)$ with normal cognition, 12 with $\mathrm{MCl}(21.1 \%)$ and 7 with dementia (12.3\%). Patient feedback was very positive. Patients worried about their cognitive decline were reassured if their scores were within normal range. Patients falling into the $\mathrm{MCl}$ range were followed-up in 6 months' time for re-testing and patients with dementia were referred to a separate specialist in neurology for independent testing. Based on these findings, sample size calculations demonstrated that 225 patients will be required for the validation study ( 75 normal cognition, $75 \mathrm{MCl}, 75$ dementia).

Conclusion: This pilot study has demonstrated that the QMCI-G screen can be used to identify normal cognition, mild cognitive impairment and dementia in German-speaking patients. It has also provided preliminary data for our validation study where $\mathrm{QMCl}-\mathrm{G}$ and MOCA results from our patients will be compared to identify optimal cutoff scores for each group. Further investigation is ongoing and results are expected mid-2018. 


\section{PS045}

Vier systematische Übersichtsarbeiten zur Pharmakotherapie bei funktionell eingeschränkten älteren Patienten mit arterieller Hypertonie und Diabetes mellitus Typ 2

Viktoria Mühlbauer'; Claudia Bollig²; Simone Brefka'; Gabriel Torbahn³; Dhayana Dallmeier'; Sebastian Voigt-Radloff'; Michael D. Denkinger ${ }^{1}$

'Agaplesion Bethesda Klinik Ulm / Geriatrisches Zentrum Ulm/Alb-Donau; ${ }^{2}$ Cochrane Deutschland, Universitätsklinikum Freiburg; ${ }^{3}$ Cochrane Deutschland, Universitätsklinikum Freiburg / Institut für Biomedizin des Alterns, Friedrich-Alexander-Universität Erlangen-Nürnberg; ${ }^{4}$ Cochrane Deutschland, Universitätsklinikum Freiburg / Zentrum für Geriatrie und Gerontologie Freiburg, Universitätsklinikum Freiburg

Hintergrund und Fragestellung: Geriatrische Patienten sind nicht primär über das chronologische Alter, sondern über Mehrfacherkrankungen und multiple funktionelle Einschränkungen charakterisiert. Die Pharmakotherapie des älteren, funktionell eingeschränkten Patienten stellt eine große Herausforderung dar, die meisten Leitlinien enthalten dazu keine Empfehlungen. Folglich sind Therapieentscheidungen mit großen Unsicherheiten verbunden, denn sie basieren entweder auf indirekter Evidenz aus Studien mit Patienten ohne Funktionseinschränkungen oder ausschließlich auf der klinischen Expertise des Arztes. Ziel des Projekts "Medikation und Lebenssituation im Alter" ist die systematische Aufarbeitung der Evidenz zur medikamentösen Therapie häufiger Erkrankungen (u.a. Diabetes mellitus Typ 2, arterielle Hypertonie) älterer, funktionell eingeschränkter Patienten.

Methoden: In vier separaten systematischen Suchen wurden randomisierte (RCT, bis März 2017) und prospektive nicht-randomisierte klinische Studien (prospektive non-RCT, bis Juli 2017) zur Pharmakotherapie bei Hypertonie und Diabetes Mellitus gesucht. Zusätzlich zu den Datenbanken Medline, Embase und Central wurden die Referenzlisten eingeschlossener Studien durchsucht. Eingeschlossen wurden Studien mit älteren, an Hypertonie bzw. Diabetes mellitus Typ 2 erkrankten Patienten, die zur Baseline im Durchschnitt einen mindestens moderat eingeschränkten Funktionsstatus aufwiesen. Mögliche Vergleiche waren Antihypertensiva bzw. Antidiabetika versus Placebo, keine oder eine andere medikamentöse Behandlung. Vergleiche verschiedener Blutdruckziele bzw. Blutzuckerziele wurden ebenso eingeschlossen. Die Auswahl, Datenextraktion und Qualitätsbewertung der Studien wurde jeweils von zwei Reviewern unabhängig voneinander durchgeführt. Konflikte wurden durch Diskussion oder durch eine dritte Person gelöst. Die Bewertung der Studienqualität erfolgte mit dem Cochrane Risk of Bias Tool für randomisierte und mit ROBINS-I für nichtrandomisierte Studien.

Vorläufige Ergebnisse: Aus 15329 Treffern bei RCTs zu Hypertonie wurden 35 potentiell relevante Zitate ausgewählt, bei den prospektiven non-RCTs aus 4570 Treffern 36. Analog wurden bei Diabetes Mellitus 44 potentiell relevante Zitate zu RCTs aus 12955 Treffern und zu prospektiven non-RCTs 113 aus 3933 Treffern identifiziert. Die Auswahl der Volltexte sowie die Datenextraktion und Bewertung der Primärstudien erfolgt im Winter 2017/2018 und wird auf dem DGIM Kongress 2018 präsentiert. Systematische Übersichtsarbeiten zu weiteren Indikationen sind in Planung.

\section{PS046}

Design und vorläufige Ergebnisse der Studie „Rolle der multidimensionalen Prognoseberechnung während der Hospitalisierung älterer, multimorbider Patienten"

Lena Pickert'; Anna Maria Meyer²; Ingrid Becker²; Thomas Benzing ${ }^{2}$; Maria Cristina Polidori ${ }^{1}$

${ }^{1}$ Uniklinikum Köln, Köln; ${ }^{2}$ Uniklinik Köln, Köln;

Hintergrund und Ziel: Die Erhaltung der Selbstständigkeit und Entlassung zurück ins häusliche Umfeld haben bei geriatrischen Patienten oberste Priorität. Ziel der Studie ist es, prognoserelevante Veränderungen, die Einfluss auf eben genannte Ziele nehmen, im Verlauf der Hospitalisierung zu identifizieren und deren Aussagekraft auf Entlassungsabläufe und klinische Entscheidungen zu untersuchen.

Methoden: Ältere (70+), multimorbide Patienten, aufgenommen in die Klinik II für Innere Medizin des Universitätsklinikums Köln, werden konsekutiv in die Studie aufgenommen. Bei Aufnahme, Entlassung und in der erwarteten Mitte der Hospitalisierungszeit wird ein Multidimensionaler Prognostischer Index (MPI) berechnet, basierend auf einem geriatrischen Assessment (Comprehensive Geriatric Assessment, (GA). Der MPI ist als Risikoindex in Bezug auf die Hospitalisierungsrate sowie die 3-Monats- und 1-Jahres-Mortalitätsrate der Patienten zu verstehen. Der MPI wird mittels acht verschiedener Items berechnet: 1. Cumulative Illness Rating Scale-Comorbidity Index (CIRS-Cl), 2. Activities of daily living (ADL), 3. Instrumental activities of daily living (IADL), 4. Mini Nutritional Assessment (MNA), 5. Short portable mental status questionnaire (SPMSQ), 6. Exton Smith Scale (ESS), 7. Medikamentenanzahl und 8. Lebensumstände des Patienten. Die Einteilung erfolgt in eine niedrige (0-0.33), mittlere (0.34-0.66) und hohe (0.67-1) Risikogruppe.

Ergebnisse: Achtzig von geplanten 200 Patienten (80,4 \pm 5.4 Jahre, $60 \%$ männlich und $40 \%$ weiblich) wurden zwischen Juni 2017 und November 2017 rekrutiert. Die häufigsten geriatrischen Syndrome waren Inkontinenz und Instabilität. Vorläufige Ergebnisse zeigen, dass sich der MPI bei 50\% der Patienten nach der Hälfte der Hospitalisierungszeit verglichen mit der Aufnahmesituation verbessern konnte, bei $25 \%$ hin zur nächst niedrigeren Risikogruppe des MPI. Bei 10\% zeigt sich eine Verschlechterung des MPI mit Zuordnung zur nächst höheren Risikogruppe. Bei Entlassung konnte bei 30\% der Patienten eine Verringerung des MPI im Vergleich zum CGA in der Hälfte der Hospitalisierungszeit gesehen werden, bei 15\% kam es zu einer Erhöhung des MPI.

Zusammenfassung: Die Erhebung eines zusätzlichen CGA in der erwarteten Mitte der Hospitalisierungszeit ist eine Möglichkeit, die Prognoseeinschätzung des Patienten zu evaluieren und klinische Entscheidungen daran anzupassen. Das Entlassungsmanagement kann bei Prognoseverbesserung forciert werden und es können bei Veränderungen des Zustands frühzeitig therapeutische Maßnahmen zur Erhaltung der Selbstständigkeit der Patienten ergriffen werden. Weitere Forschung ist nötig, um den Zeitpunkt des mittleren CGA für alle Patienten individuell abhängig von deren stationärer Liegedauer zu berechnen und die Aussagekraft für die klinische Entscheidungsfindung und das Entlassungsmanagement in Bezug auf die geriatrischen Patienten interpretieren zu können. 


\section{PS047}

Analyse erythrozytärer Parameter in einer deutschen Population $>60$-jähriger

Gabriele Roehrig-Herzog ; Kai Gutensohn²; Thomas Nebe ${ }^{3}$

${ }^{1}$ MVZ Medicum Köln-Ost, Köln; ${ }^{2}$ aescuLabor Hamburg, Hamburg; ${ }^{3} \mathrm{Hae}-$ matologie Labor Mannheim, Mannheim

Obwohl Anämie unter älteren und hochaltrigen Patienten eine hohe Prävalenz hat, ist die Datenlage hinsichtlich peripher-zellulärer Parameter in dieser Patientengruppe dünn. Die Frage nach altersphysiologischen Hämoglobin-Referenzwerten wird seit Jahren wissenschaftlich diskutiert, doch gibt es bisher keine Daten zu Referenzwerten für andere erythrozytäre Parameter wie $\mathrm{MCV}, \mathrm{MCH}$ und $\mathrm{MCHC}$. Die vorliegende retrospektive epidemiologische Querschnittuntersuchung wurde in Zusammenarbeit zwischen dem Arbeitskreis Labor der DGHO und der AG Anämie der DGG durchgeführt und hatte das Ziel, erythrozytäre Laborparameter unter ambulanten, hämatologisch gesunden Patienten $>=60$ Jahre zu analysieren.

Die Studiendaten entstammen der Datenbank eines deutschlandweit arbeitenden Labors aus dem Zeitraum 1 .Januar bis 31. Dezember 2015. Einschlusskriterien waren Alter $>=60$ Jahre, Normalwerte für CRP, TSAT, Retikulozyten, LDH, Haptoglobin und löslichem Transferrin Rezeptor; Ausschlusskrietrien waren GFR $<60 \mathrm{ml} / \mathrm{min}$ und fehlende Einschlusskriterien; primäres Studienziel war die Analyse der mittleren Hämoglobinwerte, sekundäres Studienziel war die Analyse der weiteren erythrozytären Parameter des peripheren Blutes. Von 30,611 Patienten > 60 Jahre konnten 4,641 Patienten in die Untersuchung eingeschlossen werden. Sie wurden eingeteilt in die Altersgruppen 60-69 Jahre (2,094 Patienten), 70-79 Jahre (2,171 Patienten), 80-89 Jahre (360 Patienten), >90 Jahre (16 Patienten). Die statistische Auswertung ergab, dass alle Parameter innerhalb der von der DGHO empfohlenen Referenzbereiche blieben. Die Hämoglobinwerte blieben oberhalb der WHO Grenzwerte zur Anämiedefinition. Die Ergebnisse bestätigen sowohl die WHO Grenzwerte als auch die Ergebnisse vorangehender Untersuchungen an nicht-deutschen Patientenkollektiven, so dass den vorliegenden Daten zufolge keine altersspezifische Anpassung der erythrozytären Parameter für Patienten $>60$ Jahre in Deutschland erforderlich wird.

\section{PS048}

\section{Ringelröteln im Alter - ein Fallbericht}

Claudia Schinköthe; Moritz Klugmann; Frank Mickley; Ingolf Bogner

Klinikum Sankt Georg gGmbH, Leipzig

Hintergrund: Parvovirus B19 wurde 1974 entdeckt und ist ein humanpathogenes Einzelstrang-DNA-Virus. Es wird durch Tröpfcheninfektion übertragen. Ca. 70\% der 65-Jährigen in Deutschland sind seropositiv. Die Infektion tritt mit meist leichtem und grippalem Verlauf im Kindesalter auf. Für Immunsupprimierte und Schwangere drohen besondere Risiken (u.a. aplastische Krise, Spontanabort, Hydrops fetalis). Fallberichte aus der Geriatrie sind rar und berichten über einen SLE-ähnlichen Verlauf (Federici et al. 2007).

Kasuistik: Wir berichten über eine 88-Jährige mit schwerer hämolytischer Anämie (Hkt 0,25; MCV 84 fl; LDH 8,6 $\mu \mathrm{mol} /$ I $^{*}$; Haptoglobin 3005, AT III, Thrombozyten und Fibrinogen normal, Fibrinmonomere negativ). Nach Absetzen des Vitamin K-Antagonisten, iv Gabe von Gerinnungsfaktoren (PPSB) und Transfusion von 2 Erythrozytenkonzentraten erholte sich die Patientin vollständig. Eine medikamentöse, autoimmune oder maligne Ursache der Hämolyse wurde ausgeschlossen.
Schlussfolgerung: Trotz hoher Durchseuchung treten „Kinderkrankheiten" auch bei geriatrischen Patienten auf und verlaufen dann schwer und komplikationsreich. Bei akuter Hämolyse und Hämorrhagien sollte daher bei geriatrischen Patienten auch an die Infektion mit Parvovirus B19 gedacht werden.

\section{PS049}

Anwendung des ,Geriatic 8 Screening Tools' (G8) bei Krebspatient/-innen - eine systematische Übersicht von Primärstudien

Thomas Strauß ${ }^{1}$; Gabriel Torbahn'; Cornel C. Sieber ${ }^{2}$; Dorothee Volkert'; Eva Kiesswetter ${ }^{1}$

'Institut für Biomedizin des Alterns, Friedrich-Alexander-Universität Erlangen-Nürnberg; ${ }^{2}$ Institut für Biomedizin des Alterns, FriedrichAlexander-Universität Erlangen-Nürnberg / Barmherzige Brüder Krankenhaus, Regensburg

Einführung: Zur Einschätzung gesundheitlicher, psychosozialer und funktioneller Ressourcen und als Grundlage für die Therapieplanung wird bei geriatrischen Patienten ein umfangreiches geriatrisches Assessment (Comprehensive Geriatric Assessment, CGA) durchgeführt. Um geriatrische Krebspatienten zu identifizieren, die von einem CGA profitieren könnten, wurde das G8 Screening Tool entwickelt.

Ziel: Die Arbeit soll eine systematische Übersicht der Charakteristika sowie der Ergebnisse der Primärstudien geben, in denen das G8 bisher bei Krebspatienten eingesetzt wurde.

Methodik: Im Juni 2016 wurde eine systematische Literaturrecherche in den Datenbanken MEDLINE, EMBASE, CENTRAL und CINAHL sowie in den Studienregistern 'Clinicaltrials.gov', 'International Clinical Trials Registry Platform' (ICTRP) der WHO und 'Deutsches Register Klinischer Studien' (DRKS) durchgeführt. Anhand festgelegter Einschlusskriterien (Primärstudie mit Krebspatienten, Verwendung des G8) wurden Titel/Abstracts und ggf. Volltexte von 2 Personen unabhängig auf ihre Eignung geprüft und anschließend relevante Daten extrahiert. Konflikte wurden durch Diskussion oder eine dritte Person gelöst. Die Methodik wurde in einem Studienprotokoll a priori festgelegt.

Ergebnisse: Es wurden 4683 Studien identifiziert, 594 wurden im Volltext auf Einschluss geprüft und 35 eingeschlossen. Die Mehrzahl der Studien $(n=25)$ wurde in Westeuropa durchgeführt. Das mittlere Alter der Patienten lag zwischen 72-83 Jahren. Hinsichtlich Krebsart und -therapie variieren die Studien stark. In 6 Studien wurden nur Patienten mit einer Krebsart eingeschlossen und in 7 Studien gab es nur eine Behandlungsoption (z. B. Chemotherapie, chirurgischer Eingriff). Der Anteil von Patienten mit Beeinträchtigung ( $\mathrm{G} 8 \leq 14)$, berichtet in 29 Studien, lag zwischen $25.6 \%$ und 90.8\%. Der mittlere G8-Score, berichtet in 12 Studien lag zwischen 10.0 und 14.2 von 17 erreichbaren Punkten. In 28 Studien wurde das G8 mit mindestens einem zweiten (Frailty-) Instrument (z. B. Vulnerable Elders Survey (VES-13), Groningen Frailty Index (GFI)), dem Ergebnis eines CGA oder einer modifizierten G8-Version ( 6 anstelle von 8 Items) verglichen. Die zum Vergleich herangezogenen CGAs unterschieden sich hinsichtlich der eingesetzten Instrumente (z. B. Mini-Nutritional Assessment (MNA), Activities of Daily Living (ADL)) und Grenzwerten zur Definition einer Beeinträchtigung. In 15 Studien wurde die prognostische Aussagekraft (z. B. Überlebenszeit, funktioneller Abbau) untersucht.

Schlussfolgerung und Ausblick: Die untersuchten Kollektive weisen eine hohe Heterogenität auf, sodass die Ergebnisse nicht vergleichbar sind. Zwar liegen zahlreiche Studien vor, die den G8 mit anderen Instrumenten vergleichen, allerdings kann eine abschließende Beurteilung seiner Validität aufgrund eines fehlendenden Goldstandards und der Nutzung unterschiedlicher Grenzwerte nicht erfolgen. 
Interessenkonflikt: Das Projekt wurde finanziell ermöglicht von Nestec S.A.

\section{PS050 \\ Visfatin in Knochenhomöostase von Patienten mit Arthrose und Osteoporose}

Lali Tsiklauri'; Janina Werner'; Klaus Frommer'; Stefan Rehart ${ }^{3}$; Sabine Wenisch ${ }^{2}$; Elena Neumann'; Ulf Müller-Ladner ${ }^{1}$

${ }^{1}$ Campus Kerckhoff, Bad Nauheim, Justus-Liebig-Universität Gießen; ${ }^{2}$ Justus-Liebeig-Universität Gießen; ${ }^{3}$ Agaplesion Markus-Krankenhaus, Frankfurt

Osteoporose ist eine altersbedingte, systemische Knochenerkrankung die sich durch verminderte Knochendichte und -qualität kennzeichnet. Mit zunehmendem Alter steigt der Anteil des Knochenmarkfettgewebes. Das osteoporotisch veränderte Hartgewebe wird somit direkt von den Adipozyten des Knochenmarks begrenzt. Die mesenchymale Stammzellen (MSC) können sowohl zu Adipozyten als auch für Knochenhomöostase relevanten Zellen wie Osteoblasten differenzieren. Angesichts der hohen sekretorischen Aktivitäten der Adipozyten, beeinflussen die adipozytäre Sekretionsfaktoren wie Adipokine (Visfatin, Leptin und Resistin) möglicherweise die Differenzierung der MSCs und tragen zum fortschreitenden Knochenverlust im Rahmen der Osteoporose bei. Das Expressionsprofil der Adipokine im Knochengewebe und ihre Einfluss während der Differenzierung von MSC wurde analysiert.

Nach der Isolierung von RNA aus Spongiosa humaner Hüftköpfe von Patienten mit Schenkelhalsfraktur (FF) oder mit Arthrose (OA) sowie aus den differenzierten MSC wurden die Genexpressionsanalysen mittels Real-Time PCR durchgeführt. Für die Spongiosa-Transfer-Experimente wurde die Spongiosa gereinigt und sterilisiert. Die Differenzierung von MSC erfolgte über 21 Tagen mit bzw. ohne Zugabe von Adipokinen. Die Freisetzung von Entzündungsmediatoren sowie MMP13 wurde mittels ELISA gemessen. Alizarin Red S Färbung diente zur Detektion von mineralisierter Matrix.

Visfatin wurde signifikant vermehrt und Resistin signifikant geringer in der FF-Gruppe exprimiert. Leptin war dagegen nicht unterschiedlich exprimiert. Die Supplementierung von Visfatin führte zu einem signifikanten Expressionsanstieg von MMP13 (zB. Tag 21: 104-fach) sowohl auf mRNA als auch Proteinebene während der adipogenen Differenzierung unter den Standard Zellkulturbedingungen. Im Vergleich zu Plastik war Visfatin-induzierte $\mathrm{MMP}_{13}$ Expression während der Adipogenese auf Spongiosa-Fragmente deutlich erniedrigt. In osteogen differenzierten Zellen reduzierte Visfatin die Expression von MMPs (z. B. MMP13 Tag 21: -3,18-fach) und TIMPs (z. B. TIMP2 Tag 21: $-4,3-f a c h)$. Außerdem konnte eine verstärkte Mineralisierung durch Visfatin in Rahmen der Osteogenese nachgewiesen werden, wobei die Kollagen Typ 1 Expression durch Visfatin signifikant herabgesetzt war (z. B. Tag 21: -4,6-fach). Visfatin induzierte die Expression von IL-6, IL-8 und MCP-1 sowohl während der adipogenen als auch osteogenen Differenzierung. Hierbei reduzierte die Spongiosa die Visfatin-vermittelten Effekte auf die Freisetzung von Entzündungsmediatoren während der adipogenen Differenzierung.

Visfatin-induzierte Matrixmineralisierung und Reduktion von Kollagen. Eine Expression kann zur erhöhten Knochenbrüchigkeit führen. Außerdem kann Visfatin durch die Induktion von proentzündlichen Faktoren den osteoklastären Knochenabbau begünstigen. Die Visfatin-induzierte Dysregulation der MMP- und TIMP-Expression beeinträchtigt möglicherweise den Knochenumbau an den Knochen-Knochenmark-Grenzflächen.

\section{Ernährungsmedizin / Mikrobiom}

\section{PS051 \\ Fäkale Mikrobiotatherapie als Therapieoption bei einem Clostri- dium difficile-induzierten toxischen Megacolon}

Peter Konturek'; Walburga Dieterich ${ }^{2}$; Thomas Hess'; Markus F. Neurath² Yurdagül Zopf ${ }^{2}$

'Thüringen Klinik Saalfeld, Akad. Lehrkrankenhaus Univ. Jena, Saalfeld; ${ }^{2}$ Friedrich-Alexander-Universität Erlangen-Nürnberg, Erlangen

Die Clostridium-difficile-assoziierte Colitis gehört zu den wichtigsten nosokomial übertragenen Infektionen. Die Studien der letzten Jahre zeigten, dass eine Clostridium-difficile-Colitis effektiv mit einer fäkalen Mikrobiota-Therapie (FMT) behandelt werden kann. Fallbeschreibung: Wir berichten über einen 70jährigen männlichen Patienten, der auf unserer Intensivstation mit akutem toxischen Megacolon aufgenommen wurde. In der Notfall-Endoskopie zeigte sich das Bild einer ausgeprägten pseudomembranösen Colitis.

Es wurde eine Dekompressionssonde im Colon platziert und anschließend erfolgte eine antibiotische Therapie mit Vancomycin (4x500 mg/ Tag als Einlauf) und Metronidazol (3×500 mg/Tag i.v.). Diese Therapie führte zu keiner nennenswerten Besserung des klinischen Krankheitsbildes, so dass wir uns zur Durchführung einer fäkalen Mikrobiota-Therapie entschlossen haben. An den Tagen o, 1 und 7 erfolgte die Infusion von jeweils $200 \mathrm{ml}$ Stuhltransplantat über die Dekompressionssonde in das Colon.

Bereits nach der ersten FMT kam es zur klinischen Besserung und zu einer signifikanten Rückbildung des toxischen Megacolons im CT-Abdomen und einem ausgeprägten Rückgang der Entzündungsparameter. Parallel dazu erfolgte die Untersuchung der Komposition des Darmmikrobioms vor und nach FMT mittels 16S-RNA-Sequenzierungsanalyse. Auf Phylum-Ebene zeigten sich nach der FMT eine Zunahme von Bacteroidetes sowie eine deutliche Abnahme von Proteobacteria. Auf Familien-Ebene sahen wir nach FMT eine Zunahme von Bacteroidaceae und Porhyromonodaceae sowie eine Abnahme von Enterobacteriaceae. Schlussfolgerung: 1) Die FMT kann bei akutem toxischen Megacolon als wirksame therapeutische Option eingesetzt werden; 2) Die FMT hat einen günstigen Einfluss auf die kompositionelle Veränderung des Darmmikrobioms.

\section{PS052 \\ Erfassung und Optimierung des Ernährungsverhaltens von onkologischen Patienten unter Verwendung einer Smartpho- ne-App (OncoFood)}

Till Orlemann'; Bjørn Zenker ${ }^{2}$; Dejan Reljic'; Julia Meyer'; Jana Oberländer'; Hans Joachim Herrmann'; Björn Eskofier ${ }^{3}$; Markus Neurath'; Yurdagül Zopf ${ }^{1}$

'Universitätsklinikum Erlangen - Medizinische Klinik 1; ${ }^{2}$ Lehrstuhl für Informatik, Friedrich-Alexander-Universität Erlangen Nürnberg; ${ }^{3}$ Lehrstuhl für Mustererkennung, Friedrich-Alexander Universität Erlangen-Nürnberg

Hintergrund: Die Prävalenz einer Mangelernährung ist bei Krebspatienten mit $30-80 \%$ sehr hoch. Aufgrund der tumorbedingten Anorexie und katabolen Prozesse liegt teilweise ein massives Energiedefizit vor, so dass diese Patienten unter einer ungewollten Gewichts- und Muskelmassenabnahme leiden. Eine leitlinienkonforme proteinreiche Ernährung kann den muskulären Abbau stoppen und die Prognose der Patienten positiv beeinflussen. In der klinischen Praxis ist das zeitliche und personelle Kontingent der Ernährungsmedizin häufig sehr begrenzt. Das Ziel unserer Studie war es, die Einsatzfähigkeit eines Er- 
nährungs-Apps (EA) zur Therapieüberwachung einer Ernährungstherapie bei Krebspatienten zu untersuchen. Mit dieser ersten App-Generation soll die Alltagstauglichkeit und Compliance dieser digitalen Ernährungsbetreuung erfasst werden.

Methode: Zu Beginn wurden 1400 Freiburger-Ernährungsprotokolle (FB-EP) von Krebspatienten hinsichtlich der Ernährungsgewohnheiten ausgewertet. Dies diente als Grundlage zur Erstellung einer EA Die Anforderungen an die App waren: einfache Bedienung, Wiedergabe der aufgenommenen Nährstoffe und Angabe der erreichten Tagesziele. Es wurden 39 Tumorpatienten in die Studie eingeschlossen. Alle Patienten erhielten zu Beginn eine Nährstoffanalyse und eine Ernährungsberatung. Ein Energie- und Nährstoffziel wurde definiert. Nach vier Wochen wurde untersucht, ob die Ernährungsziele erreicht wurden. Die Interventionsgruppe $(n=12)$ erhielt zusätzlich die EA. So konnten die definierten Ernährungsziele täglich über die App vermittelt werden. Nach vier Wochen wurden die Nährstoffanalyse, sowie die App-Daten ausgewertet.

Ergebnisse: Die Auswertung der FB-EPs zeigte, dass nicht alle von Tumorpatienten bevorzugten Lebensmitteln aufgelistet sind. Die Evaluierung der Top 25 angegebenen Lebensmittel zeigte auf, dass bei Tumorerkrankung scheinbar keine Fleischprodukte bevorzugt werden. Ebenfalls wurden die 200 am häufigsten verzehrten Lebensmittel evaluiert und deren Verfügbarkeit im FB-EP überprüft. Insgesamt 31 der 200 ausgewählten Lebensmittel kommen nicht im FB-EP vor. Diese Datenbasis wurde zur Entwicklung der App daher optimiert. Der Vergleich einer konventionellen Ernährungstherapie mit einer speziellen EA konnte zeigen, dass die Ernährungsziele der App-Probanden besser erzielt werden konnten. Insbesondere die kalkulierte Proteinzufuhr wurde tendenziell signifikant $(p=0,064)$ besser erzielt. Bei den Kontrollpatienten zeigte sich ein Rückgang der Makronährstoffzufuhr. Bei den App-Patienten wurde eine signifikante Gewichtszunahme $(p=0,045)$ und ein signifikanter Zuwachs an skelettale Muskelmasse $(p=0,009)$ erreicht.

Zusammenfassung: Wir konnten zeigen, dass mit Hilfe einer EA die Ernährungsziele bezogen auf die Nährstoffzufuhr signifikant besser erreicht und die Körperzusammensetzung stabilisiert werden kann. Die Ergebnisse müssen nun in einem größeren Kollektiv bestätigt werden.

\section{PS053 \\ Refeeding Syndrom und somatische Komplikationen bei Ano- rexia nervosa - eine Kasuistik}

Martina Platzer; Frederike Fellendorf; Theresa Lahousen-Luxenberger; Hans-Peter Kapfhammer

\section{Medizinische Universität Graz, Graz, Austria}

Das Refeeding Syndrom ist ein Zustandsbild, das entsteht, wenn nach längerer Zeit der Unterernährung normale Mengen an Nahrung zu rasch zugeführt werden. Es ist durch massive Störungen des Elektrolythaushaltes, Vitaminmangelzustände sowie Wasser- und Natriumretention gekennzeichnet und potenziell lebensbedrohlich. Neben onkologischen PatientInnen während einer Chemotherapie, mangelernährten geriatrischen PatientInnen und PatientInnen nach bestimmten Operationen, sind PatientInnen mit Anorexia nervosa besonders gefährdet, eine Refeeding-Syndrom zu entwickeln. Da bei schwerer Anorexie der Kostaufbau und die Gewichtszunahme initial im Vordergrund der Therapie stehen, ist es besonders wichtig, bei dieser Patientengruppe ein Refeeding-Syndrom zu vermeiden bzw. ein bereits bestehendes zu erkennen und richtig zu behandeln.Wir berichten hier über eine 20-jährige Patientin, die seit ihrem 12.Lebensjahr an einer Anorexie leidet und aufgrund des Vorliegens einer vi- talen Gefährdung mittels Polizeibegleitung an unsere psychiatrische Abteilung gebracht wurde. Zum Zeitpunkt der Aufnahme wog die Patientin ca. 22 Kilogramm, was bei einer Körpergröße von etwa 160 Zentimetern einem Body Mass Index von 8,6 und damit einem massiven Untergewicht entsprach. Die Patientin entwickelte anfänglich ein Refeeding-Syndrom mit Elektrolyt-Entgleisungen und massiven Ödemen und, nach dessen erfolgreicher Behandlung, zahlreiche weitere somatische Komplikationen, die eine enge Zusammenarbeit mit der Abteilung für Innere Medizin erforderten.Die Patientin konnte nach fast fünf-monatigen Aufenthalt auf unserer Akut-Station mit einem Gewicht von 30,5 Kilogramm entlassen werden.

\section{PS054}

Proteinreiche Ernährung und Ganzkörper-Elektromyostimulation bei Hämatologisch-onkologischen Patienten: Eine neue Option?

Dejan Reljic; Hans Joachim Herrmann; Markus F. Neurath; Yurdagül Zopf

Universitätsklinikum Erlangen - Medizinische Klinik 1

Fragestellung: Patienten mit hämatologisch-onkologischer Erkrankung haben aufgrund der tumorbedingten systemischen Inflammation ein erhöhtes Morbiditäts- und Mortalitätsrisiko. Einzig kurativer Ansatz ist häufig eine Knochenmarks/-Blutstammzelltransplantation. Die Transplantation stellt für den Körper eine hohe Stresssituation dar, so dass eine erfolgreiche Transplantation abhängig von der Körperkonstitution und dem Gesamtzustand des Patienten ist. Durch den Einsatz eines Bewegungstrainings konnte bereits gezeigt werden, dass der klinische Verlauf des Patienten deutlich positiv beeinflusst werden kann. Körperlich schwache Patienten können jedoch an konventionellen Bewegungstherapien nicht teilnehmen. Das Ziel der vorliegenden Studie war es daher, diesen Patienten ein innovatives Krafttraining, eine Ganzkörper-Elektromyostimulation (EMS), anzubieten um Muskelmasse, -kraft und -funktionalität zu verbessern.

Methoden: Es wurden 32 Patienten unter einer aktiven tumorspezifischen Therapie in die Studie eingeschlossen. Die Patienten erhielten gemäß der Empfehlung der Deutschen Gesellschaft für Ernährungsmedizin für onkologische Patienten eine Ernährungstherapie mit > $1 \mathrm{~g}$ Protein/kg KG/d und zusätzlich ein Muskeltraining mittels EMS 2X/ Woche mit jeweils 20 Minuten Applikationsdauer. Die Studiendauer betrug 12 Wochen. Hauptendpunkt der Studie war die Zunahme an Muskelmasse. Nebenendpunkte war eine Muskelkraftzunahme und Verbesserung der Funktionalität.

Ergebnisse: Es konnten bisher 16 Männer und 16 Frauen mit einem mittleren Alter von 54,3 \pm 17,6 Jahren in die Studie eingeschlossen worden. 23 Patienten erhielten eine Ganzkörper-EMS Behandlung, kombiniert mit einer proteinreichen Ernährung. 9 Patienten dienten als Kontrolle, die nur die Ernährungstherapie erhielten. Alle Patienten befanden sich unter einer Radio-, Chemo- oder/und einer Immun-/ Antikörpertherapie. Die Interventionsgruppe entwickelte nach dem dreimonatigen Krafttraining signifikant mehr Skelettmuskelmasse $(p=0,045)$ und weniger Fettmasse $(p=0,009)$. Die Funktionalität verbesserte ebenfalls signifikant unter der Sporttherapie.

Schlussforderung: Mit einem Ganzkörper-EMS-Krafttraining konnte trotz eingeschränkten körperlichen Status und unter medikamentöser tumorspezifischer Therapie mit kombinierter Glukokortikoidbehandlung signifikant mehr Muskelaufbau mit verbesserter Funktionalität induziert werden, als in der vergleichenden Kontrollgruppe. Interessanterweise zeigte sich zeitgleich, trotz der Glukokortikoidbehandlung, ein signifikanter Abbau der Fettmasse. Nachweislich dient das Fettreservoir nicht nur als Energiespeicher, sondern produziert Signalstoffe und sendet entzündungsinduzierende Zytokine aus. Ein 
Abbau der Fettmasse sollte daher einen positiven Einfluss auf die Inflammation haben.

\section{PS055 \\ Die Rolle von Gesundheitsaspekten in der Essenswahl selbstän- dig lebender Senioren mit funktionellen Einschränkungen}

Hanna Maria Rempe'; Eva Kiesswetter'; Dorothee Volkert'; Gudrun Sproesser $^{2}$; Cornel C. Sieber'; Ellen Freiberger ${ }^{1}$

IInstitut für Biomedizin des Alterns, Friedrich-Alexander-Universität Erlangen-Nürnberg; ${ }^{2} \mathrm{AG}$ Psychologische Diagnostik und Gesundheitspsychologie, Universität Konstanz

Hintergrund: Interventionen zur bedarfsgerechten Ernährung, die für ältere Menschen einen Beitrag zum Erhalt von Gesundheit und zur Prävention von Mangelernährung leisten können, setzten meist ein gesundheitsorientiertes Essverhalten der Patienten voraus. Bislang ist jedoch wenig bekannt, wie stark Gesundheitsaspekte eine Rolle in der Essenswahl für funktionell eingeschränkte, ältere Menschen spielen und welche weiteren Motive das Essverhalten wesentlich beeinflussen. Ziele waren daher (a) die Identifikation von Motiven mit starkem Einfluss auf die Essenswahl und (b) die Untersuchung soziodemografischer und gesundheitsbezogener Faktoren im Zusammenhang mit einer schwachen gesundheitsorientierten Essmotivation (SGE).

Methoden: In diese Substudie der europaweiten, multizentrischen Interventionsstudie SPRINT-T"** wurden 166 selbständig lebenden Senioren $\geq 70$ Jahren im Raum Nürnberg mit funktionellen Einschränkungen ("Short Physical Performance Battery“ 9-3) einbezogen. Unter Anwendung des "Eating Motivation Survey" erfolgte die Erfassung 15 übergeordneter Essmotive auf einer 7-stufigen Likert-Skala von 1 "nie" bis 7 „immer zutreffend für das Essverhalten". Zusätzlich wurden Geschlecht (70,5\% weiblich), Alter (80,4 $\pm 5,2$ Jahre), Body Mass Index $(29,5 \pm 5,5)$, Lebensform, Kognitionen, Schmerz, Medikamentenstatus, subjektive Gesundheit und Gebrechlichkeitsstatus anhand der Fried-Kriterien erhoben. Neben deskriptiven Auswertungen erfolgte eine multivariate Regressionsanalyse mit dem Motiv "Gesundheit" als abhängige Variable (1. vs. 2./3. Tertil).

Ergebnisse: Das Motiv „Appetit“ $(5,4 \pm 1,4)$ hatte den größten Stellenwert in der Essenswahl der Teilnehmenden, gefolgt von "Gesundheit" $(4,7 \pm 1,6)$ und "Natürlichkeit" $(4,6 \pm 1,8)$. Die Wahrscheinlichkeit (OR) einer SGE war für männliche Teilnehmer um das 4,63-fache höher als für Frauen (95\% KI 2,15-9,97). Im Vergleich zu Teilnehmenden mit drei bis fünf Gebrechlichkeitssymptomen $(n=50)$ hatten Teilnehmende mit ein bis zwei Symptomen $(n=99)$ eine signifikant geringere Wahrscheinlichkeit für eine SGE $(\mathrm{OR}=0,20 ; 95 \% \mathrm{KI} 0,10-0,43)$. Darüber hinaus waren keine weiteren Faktoren mit einer schwachen gesundheitsorientierten Essmotivation assoziiert.

Schlussfolgerung: Der Stellenwert des Gesundheitsmotivs in der Essenswahl deutet insgesamt auf eine hohe Empfänglichkeit älterer Menschen mit Funktionseinschränkungen für gesundheitsbezogene Ernährungsinterventionen hin. Besonders für männliche und gebrechliche Senioren sollten personalisierte Strategien zum Einsatz kommen und mögliche Barrieren einer gesundheitsförderlichen Ernährung thematisiert werden. Weiterhin empfiehlt es sich, vor allem geschmackliche und genießerische Aspekte der Essenswahl in Beratungskonzepten zu berücksichtigen.

*Sarcopenia \& Physical Frailty in older people: multicomponent treatment strategies
PS056

Differences in the bacterial pattern of histamine intolerant patients

Monic Schink ${ }^{1}$; Esther Tietz ${ }^{2}$; Walburga Dieterich ${ }^{2}$; Theresa C. Pinzer ${ }^{2}$; Stefan Wirtz ${ }^{2}$; Markus F. Neurath ${ }^{2}$; Yurdagül Zopf ${ }^{2}$

'Universitätsklinikum Erlangen - Medizinische Klinik 1;

${ }^{2}$ Universitätsklinikum Erlangen

Background: Histamine intolerance (HIT) is a complex and controversially discussed disorder. An impaired degradation of oral histamine intake due to diamine oxidase (DAO) deficiency is suggested as the main cause, but other factors, including an altered gut flora, may also contribute to an elevated histamine level. We aimed to determine differences in the intestinal bacterial pattern in patients with proven histamine intolerance, in comparison to healthy persons and patients with other food intolerances.

Methods: All participants had a blood testing for total lgE, food-specific IgEs, plasma histamine and DAO serum activities for group allocation. Stool samples were collected for analysis of stool histamine and zonulin levels. The bacterial composition of the stool samples was analysed by $16 \mathrm{~s}$ rRNA sequencing using Illumina platform.

Results: A total of 64 participants ( $38.3 \pm 14.2 \mathrm{yrs}, 84 \%$ female) were included in the study, and categorized into patients with confirmed histamine intolerance $(n=8,28.9 \pm 11.2 \mathrm{yr}, 100 \%$ female), food hypersensitivity ( $n=25,41.4 \pm 12.8$ yrs, $80 \%$ female), food allergy $(n=21,41.4$ $\pm 14.9 \mathrm{yrs}, 81 \%$ female) and healthy controls $(n=10,31.3 \pm 13.9 \mathrm{yrs}, 90 \%$ female). No significant differences in stool histamine levels were observed between groups. Compared to other study groups, histamine intolerant patients showed increased levels of stool zonulin (135.7 ng/ $\mathrm{ml})$, and serum TNF-a concentrations $(7.4 \mathrm{pg} / \mathrm{ml})$. Bacterial diversity was significantly reduced in histamine intolerant patients $(p=0.019)$. Furthermore, microbiome analysis revealed elevated levels of the phylum Proteobacteria in the HIT patients (5.4\%). On family level, healthy controls showed a significantly higher abundance of Bifidobacteriaceae $(0.3 \%)$ in comparison to the other study groups $(p=0.005)$, with lowest levels in the HIT group $(0.02 \% ; p=0.036)$. In addition, significantly reduced occurrence of the genera Butyricimonas $(p=0.026)$ and Hespellia $(p=0.025)$ was observed in the HIT group. In contrast, Roseburia were significantly elevated in HIT patients ( $0.45 \% ; p=0.021$ ). No increased numbers of known histamine-producing bacteria were detected in any study group.

Conclusion: In summary, our study results suggest an alteration of the microbial composition in patients with histamine intolerance. The higher abundance of Proteobacteria and lower abundance of Bifidobacteriaceae, as well as elevated stool zonulin levels indicate a dysbiosis and impaired intestinal barrier in these patients. However, elevated amounts of stool histamine or histamine-producing bacteria may not be responsible for disease pathogenesis and further investigations are needed. 


\section{PS057}

Anwendung des Mini-Nutritional Assessment (MNA) ${ }^{\oplus}$ bei Krebspatient/-innen - eine systematische Übersicht von Primärstudien

Gabriel Torbahn'; Thomas Strauß'; Cornel C. Sieber ${ }^{2}$; Dorothee Volkert'; Eva Kiesswetter'

'Institut für Biomedizin des Alterns, Friedrich-Alexander-Universität Erlangen-Nürnberg; ${ }^{2}$ Institut für Biomedizin des Alterns, Friedrich-

Alexander Universität Erlangen-Nürnberg / Krankenhaus Barmherzige Brüder, Regensburg

Einführung: Das MNA ist ein Instrument zur Früherkennung und Erfassung von (Risiko von) Mangelernährung (ME), das für Menschen über 65 Jahre (J) unabhängig von Settings und Diagnosen entwickelt und validiert wurde und auch bei Krebspatienten angewendet wird.

Ziel: Die Arbeit soll eine systematische Übersicht der Charakteristika sowie der Ergebnisse der Primärstudien geben, in denen das MNA bisher bei Krebspatienten eingesetzt wurde.

Methodik: Im Juni 2016 wurde eine systematische Literaturrecherche in den Datenbanken MEDLINE, EMBASE, CENTRAL und CINAHL sowie in den Studienregistern 'Clinicaltrials.gov', 'International Clinical Trials Registry Platform' (ICTRP) und 'Deutsches Register Klinischer Studien' (DRKS) durchgeführt. Anhand festgelegter Einschlusskriterien (Primärstudie mit Krebspatienten, Verwendung des MNA) wurden Titel/ Abstracts und ggf. Volltexte von 2 Personen unabhängig auf ihre Eignung geprüft und relevante Daten extrahiert. Konflikte wurden durch Diskussion oder eine dritte Person gelöst. Die Methodik wurde in einem Studienprotokoll a priori festgelegt.

Ergebnisse: Es wurden 4683 Studien identifiziert, 594 wurden im Volltext auf Einschluss geprüft und 104 eingeschlossen. 64 Studien setzten die Langfassung (LF), 23 die Kurzfassung (,Short-form', SF) und 7 beide Fassungen des MNA ein. In 4 Studien wurden modifizierte Versionen (angepasst an asiatische anthropometrische Grenzwerte) verwendet und in 6 Studien war die MNA-Fassung nicht berichtet. Die Hälfte der Studien ( $n=53$ ) wurde in Europa (36 in Westeuropa) durchgeführt. In 21 Studien wurden auch Personen

\section{PS058}

Ganzkörper-Elektrostimulation in Kombination mit proteinreicher Ernährung bei Patientinnen mit gynäkologischen Krebserkrankungen im metastasierten bzw. fortgeschrittenen Stadium: ein innovatives Therapiekonzept

Yurdagül Zopf; Hans Joachim Herrmann; Markus F. Neurath; Dejan Reljic

Universitätsklinikum Erlangen - Medizinische Klinik 1

Fragestellung: Patienten mit einer Krebserkrankung weisen bereits vor Eintritt einer Kachexie häufig eine Anorexie, eine reduzierte Nährstoffaufnahme und eine gestörte Nährstoffverwertung auf. Es resultiert eine ungewollte Gewichtsabnahme und Muskelabbau, die mit einem erhöhten Risiko für eine zunehmende Morbidität und Mortalität einhergehen. Nachweislich kann eine Sporttherapie eine wichtige Maßnahme zur Verbesserung der Körperkonstitution und damit der Prognose des Patienten darstellen. Die Daten in Bezug auf eine Bewegungstherapie bei einer metastasierten Situation sind jedoch begrenzt. Dies wird begründet sein mit einer geringen körperlichen Belastbarkeit dieser Patienten, so dass eine effektive Bewegungstherapie erschwert ist.
In der vorliegenden Studie wurden daher Patientinnen in metastasierten Stadien ein innovatives Krafttraining mittels einer Ganzkörper-Elektromyostimulation (EMS) in Kombination mit einer proteinreichen Ernährung angeboten. Das Ziel war eine Verbesserung der Körperzusammensetzung mit Aufbau der Muskulatur und Verbesserung der Funktionalität.

Methode: In die Studie wurden Patientinnen mit gynäkologischen Krebserkrankungen im fortgeschrittenen Tumorstadium und/oder während einer medikamentösen tumorspezifischen Therapie eingeschlossen. Über die Studiendauer von 3 Monaten erhielten die Patientinnen gemäß der Leitlinien der Deutschen Gesellschaft für Ernährungsmedizin eine eiweißreiche Ernährung mit mindestens $>1 \mathrm{~g}$ Protein/kg KG/d und ein Muskeltraining mittels einer Ganzkörper-EMS zweimal/Woche für jeweils 20 Minuten. Hauptendpunkte der Studie waren die Erfassung des Muskelstatus, der Funktionalität und der Muskelkraft nach Abschluss der 3-monatigen Intervention. Ferner wurden der Karnofsky-Index und der ECOG erfasst um den Einfluss auf die physische Gesamtsituation zu erfassen.

Ergebnisse: Es wurden insgesamt 40 Patientinnen mit einem mittleren Alter von $53 \pm 11$ Jahre in die Studie eingeschlossen. 33 Patientinnen bekamen die kombinierte Behandlung aus EMS und proteinreiche Ernährung und 7 Patientinnen stellten die Kontrollpopulation dar, die nur eine eiweißreiche Ernährung erhielt. Die Interventionsgruppe nahm nach drei Monaten signifikant an Handkraft $(p=0.008)$ und Funktionalität (6-Minuten-Gehtest; $p=0.004$ ) zu. Die Skelettmuskelmasse zeigte nur eine tendenziell höhere Muskelmassenzunahme im Vergleich zu der Kontrollgruppe $(p=0.07)$. Bei der Interventionsgruppe verbesserte sich der Karnofsky-Index signifikant $(p=0.011)$ und tendenziell der ECOG $(p=0.066)$.

Schlussforderung: Mittels Ganzkörper-EMS konnte trotz fortgeschrittenem Krankheitsstadium ein Krafttraining mit Verbesserung der Muskelkraft, Funktionalität und des physischen Zustandes des Patienten erzielt werden. Bei zeitgleich verbessertem Karnofysky-Index kann auch von einem positiven Einfluss auf die Prognose des Patienten ausgegangen werden. Die Skelettmuskelmasse hat sich nicht signifikant gesteigert präsentiert. Dies wird aufgrund der kleinen Studienpopulation bedingt sein.

\section{Immunologie / Infektiologie I}

\section{PS059}

Antiinfektive Therapie einer rekurrenten Acinetobacter Baumannii (4MRGN, Carbapenemase-Bildner) Infektion unter "Antibiotika-Monitoring"

Julia Anraths; Hendrik Haake

Kliniken Maria Hilf GmbH, Krankenhaus St. Franziskus, Mönchengladbach

Ein 58-jähriger Patient wurde uns heimatnah aus einem thailändischen Krankenhaus zugewiesen. Dort hatte eine intensivmedizinische Therapie aufgrund eines septischen Schocks bei einer ambulant erworbenen Pneumonie stattgefunden. Aufgrund einer schweren respiratorischen Insuffizienz musste eine invasive Beatmung erfolgen, es entwickelte sich ein septisches Multiorganversagen. Im Bronchialsekret hatte sich der Nachweis eines Acinetobacter Baumannii (4MRGN) ergeben. Zum Aufnahmezeitpunkt war der Patient hämodynamisch und respiratorisch stabil sowie wach und spontanatmend. Zwei Tage später entwickelte sich ein schweres ARDS mit beidseitigen Infiltraten im CT des Thorax. Der Patient musste erneut invasiv beatmet werden, eine Bauchlagerungstherapie wurde durchgeführt. Es entwickelte sich ein septisches akutes Nierenversagen mit Indikation 
zur Hämodialyse. Wir konnten persistierend Acinetobacter Baumannii (4MRGN) im Bronchialsekret nachweisen. Eine antibiogrammgerechte Therapie mit Colistin i.v. und Tigecyclin wurde begonnen. Sämtliche weitere getestete Antiinfektiva erwiesen sich als resistent. Bei fehlender Besserung der Klinik und der Infektparameter stellten wir die Therapie auf Colistin inhalativ plus i.v., Rifampicin und Imipenem in Kombination um. Rifampicin wurde zum einen aufgrund der Suppression von Colistin-resistenten Stämmen in einer Colistin-sensiblen Population angewendet, zum anderen zur Erhöhung der Permeabilität der Zellwand des Keimes. Schon am Folgetag konnte ein Abfall des CRP-Wertes um $60 \%$ registriert werden. Es zeigte sich dann die Expression einer Carbapenemase. Wir entschieden uns bewusst für die Fortführung der Therapie mit Imipenem und ergänzten Sulbactam anstatt Rifampicin zur Reduktion der minimalen Hemmkonzentration (MHK) und zur Steigerung der Empfindlichkeit bei Carbapenemresistenz. Nach initial hochdosierter Therapie planten wir die Dosierung auf eine nierenadaptierte Dosis zu senken. Aufgrund von fehlenden Fachinformationen bezüglich der Dosierung von Colistin bei eingeschränkter Nierenfunktion sowie des persistierenden Erregernachweises nahmen wir Kontakt mit dem Institut für Biomedizinische und Pharmazeutische Forschung (IBMP) in Nürnberg auf. Es erfolgten tägliche Spiegelkontrollen unserer Antiinfektiva. Engmaschig fanden telefonische Konferenzen im Expertenteam statt, um die Antibiotika anzupassen, da bisher nur für etablierte Wirkstoffe Richtlinien vorhanden sind. Es kam zur Reduktion der Infektparameter mit persistierender Besiedelung durch den Erreger. Eine Woche später ereignete sich eine erneute Pneumonie, nun wieder mit massenhaftem Nachweis des Keims und progredienten pulmonalen Infiltraten.

Wir leiteten erneut eine Therapie nach Antibiogramm mit Colistin, Imipenem und Sulbactam ein. Darunter kam es nicht nur zur Ausheilung der Pneumonie, sondern auch zur vollständigen Eradikation des Erregers im Bronchialsekret. Der Patient konnte nach zweieinhalb Monaten zur Anschlussrehabilitation entlassen werden.

\section{PS060}

Fieber nach Thailandurlaub - Der seltene Fall einer Melioidose in Deutschland

Fanny Seiboth, Sven Kolfenbach, Daniel Ensberg, Steffen Rickes

HELIOS Bördeklinik, Klinik für Innere Medizin, Kreiskrankenhaus

Einleitung: Die Melioidose, ausgelöst durch das gramnegative, aerobe Stäbchenbakterium Burkholderia pseudomallei, wird durch Kontakt, Nahrungsaufnahme oder aerogen übertragen. Die Erreger befinden sich im Boden, in verunreinigtem Wasser sowie auf Gemüse und Früchten. Eine oberflächliche Hautläsion ist häufig die Eintrittspforte. Die Inkubationszeit liegt bei 1 bis 21 Tagen, die Infektion kann aber auch mehrere Jahre stumm verlaufen. Die Melioidose, auch "Pseudorotz" genannt, ist vor allem in Südostasien, aber auch in den tropischen Gebieten Australiens, im Westen Afrikas und in Südamerika verbreitet.

Kasuistik: Ein 51-jähriger Mann kam mit Fieber bis $40^{\circ}$ Celsius ins Krankenhaus. Zudem bestanden Unwohlsein und Abgeschlagenheit. Der Patient war vor 7 Tagen von einem zweiwöchigen Thailandurlaub zurückgekehrt. Am drittletzten Urlaubstag hatte ganztägig eine Diarrhoe bestanden. Eine ambulant durchgeführte Blutentnahme ergab massiv erhöhte Entzündungswerte mit Hinweisen auf eine bakterielle Infektion. Unter Berücksichtigung des Auslandsaufenthaltes wurde aufgrund der Schwere des klinischen Krankheitsbildes nach Entnahme von Blutkulturen sofort eine kalkulierte Antibiotikatherapie mit Levofloxacin per os begonnen. Bei Exsikkose und Fieber wurden zudem Infusionen und Antipyretika verabreicht. Unter diesen Maßnahmen kam es zur raschen Besserung der Beschwerden. In den
Blutkulturen wurde das Bakterium Burkholderia pseudomallei nachgewiesen. Nach Bestätigung des Erregers durch das Robert-Koch-Institut wurde die Diagnose einer Melioidose gestellt. Das Bakterium zeigte sich sensibel gegenüber dem verabreichten Levofloxacin. Die Infektion wurde dem Gesundheitsamt gemeldet und der Patient auf Grund der Seltenheit der Erkrankung hierzulande in einer infektiologischen Spezialabteilung angebunden. Rückblickend erinnerte sich der Patient an eine in der Zwischenzeit abgeheilte kleine interdigitale Verletzung des linken Fußes während des Urlaubs, die als Eintrittspforte für die Keime gewertet werden muss.

Schlussfolgerung: Im vorliegenden Fall handelte es sich um eine Infektionskrankheit, die in unseren Breiten selten ist und aus dem Urlaub in Thailand mitgebracht wurde. An diesem Beispiel lassen sich die Wichtigkeit einer präzisen Reiseanamnese sowie die Notwendigkeit der Blutkulturentnahme vor Beginn einer Antibiotikatherapie bei unklaren, am ehesten bakteriellen Infektionen aufzeigen. Weiterhin ist natürlich eine gründliche körperliche Untersuchung obligat, um auch kleine Verletzungen, die als Eintrittspforte für Erreger dienen können, zu finden und zu werten. An Melioidose erkranken weltweit jährlich rund 169.000 Menschen, wobei ca. 89.000 Infektionen letal enden.

\section{PS061 \\ Invasives Klebsiella pneumoniae Syndrom mit Leberabszess, beidseitiger Pneumonie, Panzytopenie und akutem Nierenver- sagen bei einem jungen in Deutschland wohnenden Asiaten}

Laurentiu Broscaru; Martin Müller; Frank Ingenabel; Vincent Golsong; Linda Balzen; Dietrich Gulba

\section{Katholisches Klinikum Oberhausen, St. Marienhospital, Oberhausen}

Ein 36-jähriger asiatischer Patient stellte sich mit seit einer Woche persistierendem Fieber, Schüttelfrost und Abgeschlagenheit sowie passageren epigastrischen Schmerzen und Erbrechen vor. Asthma bronchiale sei bekannt. Er sei zuletzt vor 6 Monaten in Shanghai gewesen. Der körperliche Befund blieb bis auf eine Tachykardie und leichten epigastrischen Druckschmerz unauffällig. Die orientierende Aufnahmesonographie wies eine wandverdickte Gallenblase ohne Steinnachweis und eine Hepatosplenomegalie nach. Laborchemisch zeigten sich initial ein CRP von $38 \mathrm{mg} / \mathrm{dl}$ und ein Procalcitonin von 13,7 $\mathrm{ng} / \mathrm{ml}$, eine Thrombozytopenie mit einem tiefsten Wert von 43000/ul, im Verlauf mit begleitender leichter Leukopenie (vorwiegend Neutrophile in der manuellen Differenzierung) und normozytärer Anämie, eine direkte Hyperbilirubinämie mit Gesamtbilirubin von maximal 3,4 $\mathrm{mg} / \mathrm{dl}$, leichtem Transaminasenanstieg, ein INR von 1,3 und ein Kreatinin von $2,4 \mathrm{mg} / \mathrm{dl}$ mit Harnstoff von $66 \mathrm{mg} / \mathrm{dl}$ und ohne Nachweis von Zylindern im Urinsediment und ohne Infektzeichen im Urin. Bei initialem Verdacht auf schwere bakterielle Sepsis erfolgte eine Infusions- sowie antibiotische Therapie mit Ceftriaxon. 3 Tage später erbrachten die Blutkulturen den Nachweis von Klebsiella pneumoniae, Ceftriaxon-sensibel. Ruhedyspnoe und Hypoxämie ( $\left.\mathrm{pO}_{2} 48 \mathrm{mmHg}\right)$ fielen ebenfalls auf, sodass der Patient Sauerstoff bekommen musste. Eine zweite Sonographie zeigte doch einen $4,7 \times 4 \mathrm{~cm}$ großen septierten Leberabszess im Übergang Segment $2 / 3$. Im CT wurden sowohl der Leberabszess als auch pneumonische Infiltrate in beiden Unterlappen, die im konventionellen Röntgen nicht nachweisbar gewesen waren, dokumentiert. Bei persistierenden nächtlichen Schüben bis $40^{\circ} \mathrm{C}$ wurde die Therapie mit Gentamycin ergänzt. Mikrobiologisch konnte die hypervirulente Form von Klebsiella pneumoniae anhand der Hypermukoviskosität (string test deutlich positiv) bestätigt werden. HIV-Test war negativ. Die Hepatitisserologie ergab eine abgelaufene Hepatitis B. Im Verlauf waren die Dyspnoe und das Fieber rückgängig und es kam ebenfalls zu einer Normalisierung der Entzündungswerte, der Thrombozyten und Leukozyten sowie der Nie- 
renretentionsparameter und des Bilirubins. Sonographisch gesteuert konnte eitriges Sekret aus dem Leberabszess gewonnen werden, die Anlage einer Drainage blieb jedoch frustran, sodass der Patient zur CT-gesteuerten Punktion verlegt wurde.Nach 7 Tagen Spülung konnte die Drainage entfernt und die antibiotische Therapie beendet werden. Der Patient war beschwerdefrei und konnte entlassen werden

\section{PS063 \\ Vaskulitis durch Kokain-Missbrauch}

Christian Dorbath; Claudius Kleinert; Martin Brzoska; Peter Grützmacher

AGAPLESION Markus-Krankenhaus, Frankfurt

Zielstellung: Bis zu 70\% des Kokains sind mit Levamisol, einem Anthelminthikum, verunreinigt. Levamisol kann eine ANCA-positive Vaskulitis mit ungewöhnlicher Antikörper-Konstellation und schweren nekrotischen Verläufen verursachen.

Ergebnisse: Bei der Patientin handelt es sich um eine 33-jährige drogenabhängige Frau mit seit 2 Jahren bekannter Livedo racemosa im Rahmen eines Kokain-Missbrauchs. Eine damals begonnene immunsuppressive Behandlung mit Steroiden und Azathioprin wurde nicht mehr eingenommen, aber der Kokain-Missbrauch fortgesetzt. Bei Aufnahme betrug die Körpertemperatur $40,4^{\circ} \mathrm{C}$, die Patientin war exsikkiert, der Schockindex war positiv. Im Bereich beider Oberschenkel sowie gluteal zeigten sich multiple bis handtellergroße nekrotische Ulcerationen sowie eine ausgeprägte lila-rötliche Verfärbung der Haut mit Blasenbildung. Zusätzlich zeigten sich auch im Gesicht livide-nekrotisch verfärbte Areale. Trotz Wundversorgung und antibiotischer Therapie kam es innerhalb von 3 Tagen zu einer fulminanten Ausdehnung mit Befall $>30 \%$ der Haut. Es wurde mit einer Steroid-Stoßtherapie begonnen, zunächst mit Methylprednisolon $500 \mathrm{mg} /$ die über 3 Tage und anschliessend Prednisolon i.v. $100 \mathrm{mg} /$ die. Zusätzlich wurde bei Nachweis von p-ANCA, MPO und PR3 sowie Kryoglobulinen eine Plasmapherese-Therapie mit 9 Behandlungen durchgeführt, worunter keine neuen Nekrosen auftraten. Die Patientin benötigte eine intensivmedizinische Versorgung mit einer Opiat-Therapie bei ausgeprägter Schmerzsymptomatik. Im Verlauf erfolgten ausgedehnte operative Debridementes mit Nekrosektomie der betroffenen Hautareale und die Anlage eines protektiven Colostomas zur Prophylaxe einer Wundinfektion. Die Patientin wurde dann zur Spalthaut-Transplantation im Bereich beider Beine und des Gesässes in ein spezialisiertes Zentrum verlegt. Da unter alleiniger Steroidtherapie neue livide Hautverfärbungen im Bereich beider Oberarme auftraten erfolgten die Rückverlegung und erneute Plasmapherese-Serien. Darunter zeigte sich wieder ein Rückgang der neu aufgetretenen Hautveränderungen. Zusätzlich wurde nach Abschluss der operativen Behandlungen und Hauttransplantationen eine Cyclophosphamid-Therapie mit $500 \mathrm{mg} / \mathrm{m} 2$ Körperoberfläche begonnen. Die Patientin stellte sich zum 2. und 3. Zyklus Cyclophosphamid mit jeweils $500 \mathrm{mg}$ pro $\mathrm{m} 2 \mathrm{KOF}$ im Abstand von 4 Wochen in unserem Zentrum vor, weitere Zyklen sind geplant. Parallel wurde die orale Prednisolon-Dosis auf zuletzt $20 \mathrm{mg}$ pro die reduziert. Die Nierenfunktion blieb stabil normwertig.

Schlussfolgerungen: Auch durch einen Kokain-Missbrauch kann eine fulminant verlaufende Vaskulitis induziert werden, welche den Einsatz einer immunsuppressiven Therapie inclusive Plasmapherese notwendig machen kann.

\section{PS064}

Azathioprine metabolite (6-TGN) levels within a defined therapeutic range are associated with lower fecal calprotectin in Crohn's disease - A retrospective cross-sectional analysis

Julian Essmann; Carsten Keil; Michael Manns; Oliver Bachmann

Medizinische Hochschule Hannover, Hannover

Background: Azathioprine (AZA) belongs to the first-line immunosuppressants for the treatment of Crohn's disease. While recent studies have reported a positive association of its active metabolite 6-thioguanine (6-TGN) with clinical outcomes, 6-TGN levels have not been correlated with surrogate markers of mucosal healing, which is an increasingly recognized therapeutic goal.

Aim: We therefore asked whether 6-TGN levels within a defined therapeutic range are associated with lower fecal calprotectin (FC) in Crohn's disease.

Methods: 6-TGN and corresponding FC levels of 96 Crohn's disease patients (Median age 31 years, $64 \%$ female) without further immunosuppressive therapy visiting the Hannover IBD outpatient clinic between 2009 and 2016 were retrospectively analysed. Calprotectin and laboratory parameters of 33 patients with TGN levels within the therapeutic range of 250 to $450 \mathrm{pmol} / 8 \times 10 \wedge 8$ red blood cells (RBCs; Estevinho MM JCC 2017) were then compared to 54 patients below and 9 patients above this range using Kruskal-Wallis test and Bonferroni correction for $p$ values. In a small sub-cohort $(n=26)$ with repeated serial 6-TGN measurements, longitudinal FC measurements were evaluated (Registered as DRKSooo13246).

Results: In the cross-sectional approach, 6-TGN levels between 31 and $780 \mathrm{pmol} / 8 \times 10^{\wedge} 8$ red blood cells (RBCs; median 6-TGN 227 $\mathrm{pmol} / 8 \times 10 \wedge 8 \mathrm{RBCs}$, interquartile range 154,5 to 312,5 ) and a mean AZA dose of $2.23 \mathrm{mg} / \mathrm{kg}$ body weight were observed. In patients with 6-TGN levels within the defined range of $250-450 \mathrm{pmol} / 8 \times 10 \wedge 8 \mathrm{RBCs}$, fecal calprotectin levels were significantly lower than in patients below this level (median FC 142,5 vs. 308,4 mg/kg, Kruskal-Wallis test, adjusted $\mathrm{p}=0,037, \mathrm{r}=0,27)$ and also than in patients above this level (median FC 142,5 vs. $559,1 \mathrm{mg} / \mathrm{kg}$, Kruskal-Wallis Test, adjusted $p=0,003, r=0,48$ ). Haemoglobin (HB) concentrations in patients within therapeutic range were significantly higher than in patients with lower metabolites (median HB 13,1 vs. 12,5, adjusted $\mathrm{p}=0.041$ ). CRP, transferrin saturation and protein levels were not different among the groups. In the small cohort that was followed over a maximum time of one year, 8/9 patients who received AZA dose escalation or initiation of allopurinol treatment achieved an increase in 6-TGN levels (median $45 \mathrm{pmol} / 8 \times 10 \wedge 8$ $\mathrm{RBC}$, range -159 to 200 ), which was paralleled by a decrease in FC (at least $-50 \mathrm{mg} / \mathrm{kg}$ ) in $4 / 9$, stagnation in $3 / 9$ and increase in $2 / 9$ (FC difference ranging from -603 to $+123 \mathrm{mg} / \mathrm{kg}$ ), but patient number was insufficient for statistical testing.

Summary: In our retrospective analysis on Crohn's disease patients receiving thiopurine monotherapy, 6-TGN levels within a defined range $\left(250-450 \mathrm{pmol} / 8 \times 10^{\wedge} 8\right.$ red blood cells) were associated with significantly lower fecal calprotectin levels as a surrogate marker for mucosal healing. A treat-to target concept directed by 6-TGN levels to reach mucosal healing in Crohn's disease appears promising, but requires prospective studies. 


\section{PS065}

Wirkung von Adipokinen und IL-17 auf synoviale Fibroblasten von Patienten mit unterschiedlichem rheumatischen Krankheitshintergrund

\author{
Klaus Frommer'; Stefan Rehart ${ }^{2}$; Michael Sauerbier ${ }^{3}$; Ulf Müller-Ladner'; \\ Elena Neumann ${ }^{1}$ \\ ${ }^{1} J u s t u s-L i e b i g-U n i v e r s i t a ̈ t ~ G i e ß e n ;{ }^{2}$ Agaplesion Markus-Krankenhaus; \\ ${ }^{3}$ Berufsgenossenschaftliche Unfallklinik Frankfurt am Main
}

Hintergrund und Zielsetzung: Die Rheumatoide Arthritis (RA) und die Psoriasisarthritis (PsA) weisen mehrere Gemeinsamkeiten auf, besitzen aber auch spezifische Unterschiede. Synoviale Fibroblasten (SF) sind zentrale Effektorzellen in der Pathophysiologie der RA. Wir stellten die Hypothese auf, dass differentielle Reaktionen der SF von Patienten mit PsA oder RA auf verschiedene Stimuli zu diesen Unterschieden beitragen könnten. Zum Beispiel ist IL-17, welches auch im Synovialgewebe exprimiert wird, von therapeutischer Bedeutung in der PsA, während es bei der RA keine vergleichbare Wirksamkeit besitzt. Bisher war IL-17 v.a. in seiner Isoform IL-17A ein therapeutisches Target bei der PsA, jedoch spielt auch IL-17F eine Rolle im Kontext der IL-23/IL-17-Achse entzündlicher Erkrankungen. Daher analysierten wir die Reaktionen von SF von Patienten mit PsA, RA oder ohne rheumatische Erkrankung auf IL-17A/F +/- TNF und ausgewählte Adipokine.

Methoden: SF wurden aus Synovium von Patienten mit PsA, RA und aus Kontrollen ohne rheumatische Erkrankung $(\mathrm{N})$ isoliert, welche sich einer Gelenkersatzoperation unterzogen. PsASF, RASF und NSF wurden jeweils mit IL-17A/F, TNF, Visfatin und Resistin stimuliert. Ein neutralisierender Anti-IL-17A Antikörper wurde eingesetzt, um die Spezifität der IL-17A Effekte zu verifizieren. Die Sekretion des proinflammatorischen Zytokins IL- 6 wurde als initialer Parameter verwendet und mittels ELISA quantifiziert.

Ergebnisse: Die Stimulierung mit Visfatin bewirkte einen Anstieg der IL-6 Sekretion in allen SF-Typen (je $n=3$ ), während Resistin keine Wirkung zeigte. Die Unterschiede in den Reaktionen zwischen den untersuchten SF-Typen waren nicht statistisch signifikant. IL-17A in Konzentrationen, wie sie in Serum oder Synovialflüssigkeit auftreten, führten bei keinem SF-Typ zu einer Induktion der IL-6 Synthese. Eine Dosis-Wirkungskurvenanalyse zeigte, dass erst beträchtlich höhere Konzentrationen an IL 17A, wie sie z.T. im Gewebe anzutreffen sind, eine Induktion der IL-6 Sekretion bewirken. Ein Anti-IL-17A Antikörper hob diese Wirkung auf, was die Spezifität des Effekts bestätigte. Die Effekte von IL-17A und IL-17F auf die IL-6 Sekretion von PsASF konnten durch Ko-Stimulierung mit TNF merklich verstärkt werden (IL-17A: 5-fach vs 113-fach; IL-17F: 1,7-fach vs 39-fach; TNF allein: 12-fach). Die Effekte waren mit IL-17A ausgeprägter als mit IL-17F und zwar sowohl mit als auch ohne TNF-Ko-Stimulierung. Für IL-17F konnte keine Wirkung auf NSF beobachtet werden.

Schlussfolgerungen: SF von RA- bzw. PsA-Patienten wurden durch die Adipokine Visfatin und Resistin bzw. IL-17A, wenn diese in Serumoder Synovialflüssigkeitskonzentrationen eingesetzt werden, nicht differentiell beeinflusst. Die Daten legen die Vermutung nahe, dass IL17 in der Pathophysiologie der RA im Gegensatz zur PsA generell nur in Verbindung mit TNF effektiv ist.
PS067

Lipid im- and exporters differentially determine macrophage dynamics and turnover in the atherosclerotic plaque

Carmen Härdtner'; Ingo Hilgendorf²; Andreas Zirlik'; Christoph Bode4; Florian Willecke ${ }^{5}$; Dennis Wolf; Peter Stachon ${ }^{5}$; Katja Grotius; Jiadai Zou; Alina Jander

${ }^{1}$ Universitäts-Herzzentrum Freiburg-Bad Krozingen, Freiburg; ${ }^{2} \mathrm{Herz}-$ zentrum Bad Krozingen; ${ }^{3}$ Universitäts Herzzentrum Standort Freiburg; ${ }^{4}$ Universitäts-Herzzentrum; ${ }^{5}$ Albert-Ludwigs-Universität Freiburg Innere Medizin III, Kardiologie und Angiologie

Background and hypothesis: Foam cell accumulation drives plaque progression. Modified lipoproteins, e.g. oxidized low-density lipoprotein (oxLDL), are internalized by scavenger receptors (SR) of macrophages in atherosclerotic plaques. ATP binding cassette (ABC) transporters, on the other hand, export excess cholesterol from foam cells. We hypothesized that cellular lipid homeostasis regulates macrophage turnover in the plaque.

Methods and results: Irradiated LDLR-/- mice were reconstituted with a mixture of CD45.1+ wildtype (WT) and CD45.2+ Msr-/-, $C_{36-/-,}$ or LysMCre/Wt:ABCA1fl/fl:ABCG1fl/fl bone marrow cells, respectively. In reconstituted chimeric mice, WT and knockout macrophages accumulated within the same plaque and were exposed to the same external stimuli under high cholesterol diet. This approach allowed us to test the effects of scavenger receptors and cholesterol exporters on macrophages in the plaque, selectively. SR deficiencies impaired lipid uptake, and reduced local macrophage proliferation by $30-50 \%$, depending on the receptor and disease stage. Accordingly, in mixed chimeras, WT macrophages outcompeted SR knockout macrophages in the atherosclerotic plaque by 2:1-6:1. In contrast, $A B C$ transporter deficiency impaired cholesterol export and slightly increased macrophage proliferation. Still, in these chimeras, WT macrophages outcompeted knockout macrophages in the atherosclerotic plaque since cholesterol overloading in knockout cells doubled their rate of cell apoptosis.

Conclusion: Our results underline the importance of local macrophage accumulation in the atherosclerotic plaque, and identify lipid uptake as driving force for macrophage proliferation.

\section{PS068}

Atorvastatin induces plaque regression by inhibiting macrophage proliferation

Carmen Härdtner'; Jan Kornemann; Katja Grotius; Peter Stachon ${ }^{2}$; Dennis Wolf; Florian Willecke'; Christoph Bode ${ }^{3}$; Andreas Zirlik'; Ingo Hilgendorf ${ }^{5}$

'Universitäts-Herzzentrum Freiburg-Bad Krozingen, Freiburg;

${ }^{2}$ Albert-Ludwigs-Universität Freiburg Innere Medizin III, Kardiologie und Angiologie; ${ }^{3}$ Universitäts-Herzzentrum; ${ }^{4}$ Universitäts Herzzentrum Standort Freiburg; ${ }^{5}$ Herzzentrum Bad Krozingen

Background and hypothesis: Statins are predominantly used in clinic for primary and secondary prevention of cardiovascular disease. They have been reported to induce plaque regression, but the underlying mechanisms remain elusive. We hypothesize that atorvastatin treatment, by lowering systemic cholesterol levels in vivo, inhibits local macrophage proliferation in the plaque and thus leading to plaque regression.

Methods and results: ApoE-/- mice and $\mathrm{APOE}^{*} 3$-Leiden.huCETP mice were fed a high cholesterol diet (HCD) containing $1.25 \%$ cholesterol for 12 weeks to induce atherosclerosis followed by a low cholesterol 
diet (LCD) containing $0.05 \%$ cholesterol for additional 4 weeks to prepare for therapeutic intervention. Here we compared the effects of continued $L C D$, with $L C D$ plus $0.01 \%$ atorvastatin, and cholesterol free diets. Atorvastatin decreased total plasma cholesterol levels by $50 \%$ in $\mathrm{APOE}^{*} 3$-Leiden.huCETP but not in unresponsive ApoE-/- mice. Only in $\mathrm{APOE}^{*}$ 3-Leiden.huCETP, therefore, statin treatment resulted in plaque regression by halting lesion growth, reducing lipid and macrophage contents by $25 \%$, and increasing fibrosis. In a series of experiments, we identified inhibition of local macrophage proliferation as the dominant mechanism of the statin induced, but lipid mediated decline in macrophage cell numbers in the plaque. Importantly, rates of macrophage proliferation in human carotid artery plaques correlated with serum LDL-cholesterol levels, in line with our experimental studies.

Conclusion: Atorvastatin reduces local macrophage proliferation in atherosclerotic lesions via lowering of cholesterol, a potent trigger of foam cell proliferation, and leads to plaque regression.

\section{PS069 \\ Streptococcus dysgalactiae sepsis with polyarthritis - think endocarditis and focus control}

Sina Helbig; Katja Wilke; Maik Stiehler; Katja de With

Universitätsklinikum Carl Gustav Carus TU Dresden, Dresden

Background: Endocarditis is typically diagnosed by bacteremia with a typical pathogen and vegetations on echocardiography. High-dose pathogen-specific antibiotics are key to treat the infection. Lack of response to therapy poses a challenge to physicians. We present a case of possible Streptococcus dysgalactiae endocarditis based on modified Duke criteria with septic arthritis of multiple joints and persistent fever despite guideline-adherent therapy.

Case: A 73-year old man with a history of atrial fibrillation, 2-chamber pacermaker, diabetes mellitus type 2 , rightsided omarthrosis presented with complaints of rigors, fever $\left(40,5^{\circ} \mathrm{C}\right)$ and recurrent syncopes of 2 days. On admission vitals were: $\mathrm{T} 38,2^{\circ} \mathrm{C}, \mathrm{BP} 155 / 70, \mathrm{HR} 110, \mathrm{O} 298 \%$ (room air), RR 19/min. Pertinent physical exam findings were moderate distress and a painful, swollen left wrist, there was no cardiac murmur. Pertinent laboratory values were leucocytes (LC) 14.410/ul, procalcitonine (PCT) $33 \mu \mathrm{g} / \mathrm{l}$, C-reactive protein (CRP) $234 \mathrm{mg} / \mathrm{dl}$, Lactate 9.3 $\mathrm{mmol} / \mathrm{L}$, eGFR 41. Chest X-ray showed no infiltrates. Empiric therapy with ampicillin/sulbactam $3 \times 3$ IV PLUS clarithromycin $2 \times 500 \mathrm{mg}$ IV was started. Blood cultures (BC) (2 sets) grew Streptococcus (S.) dysgalactiae within 6 hours. Therapy was switched to penicillin $\mathrm{G} 4 \times 5$ Mio IU IV. Arthrocentesis of the left wrist showed $3+$ granulocytes but no crystals, culture grew S. dysgalactiae. On hospital day (HD) 2 pain in the right elbow and right knee was noted. Bursitis without joint involvement was diagnosed. Transesophegeal echocardiography on HD 4 was negative for vegetations or a persistent foramen ovale. Urinary sediment showed hyaline cylinders. Abdominal sonography was negative for infarcts or abscesses. Blood cultures already sterilized on HD 2. After initial decrease of LC, PCT and CRP the fever recurred while $B C$ remained sterile. A search of further foci was pursued. Computertomography scan of the thorax and abdomen on HD 10 showed an extensive effusion in the right shoulder. Arthrocentesis and lavage was performed, revealing a cell count $>50,000 / u l$, the culture remained sterile. Now the patient showed continuous improvement. High-dose Penicillin $\mathrm{G}$ was continued for 4 weeks for possible endocarditis (modified Duke criteria: 1 major (BC), 2 minor (fever, urinary sediment)).

Discussion: The diagnosis of endocarditis should be based on the modified Duke criteria. Despite valvular vegetations being absent, the presence of multiple septic joints adds to the suggestive diagnosis of endocarditis. Drainage/lavage of foci is prudent to control and cure the infection. The sterile shoulder joint culture with however high cell count is explained by antibiotic therapy.

Conclusion: Evaluation of endocarditis should follow the modified Duke criteria. Choice and duration of parenteral antibiotic therapy should be based on guidelines. Persistent fever despite guideline-adherent therapy should prompt a search for so far undrained foci.

\section{PS070}

\section{Adipokine im Arthrose Mausmodell}

Marie-Lisa Hülser'; Carina Schreiyäck'; Yubin Luo²; Aline Bozec²; Georg Schett ${ }^{2}$; Elena Neumann'; Ulf Müller-Ladner ${ }^{1}$

1Justus-Liebig-University Gießen; ${ }^{2}$ University of Erlangen-Nürnberg

Hintergrund: Adipositas und die daraus daraus resultierenden Veränderungen sind weltweit ein Problem mit wachsender Bedeutung für Gesundheit und Lebensqualität. Diese führt auch zur Veränderung des Adipokinprofils, u.a. Adiponektin, Leptin und Visfatin, welche wiederum das Immunsystem und rheumatologische Erkrankungen wie die Osteoarthritis/Arthrose (OA) beeinflussen.

Ziele: Um das Zusammenspiel der Komorbiditäten Adipositas und OA zu untersuchen, wurden eine high-fat Diät (HFD) und ein OA-Modell (DMM: Destabilisierung der medialen Menisken) kombiniert und dabei lokale mit systemischen Veränderungen der Adipokine im Zeitverlauf untersucht und miteinander korreliert.

Methoden: $\mathrm{C}_{57 \mathrm{BI} / 6}$ Mäuse wurden mit HFD oder entsprechender ND (normale Diät) über einen Zeitraum von 3 Monaten gefüttert. Anschließend wurden DMM Operationen durchgeführt (Zeitpunkt o) und nach 4, 6 und 8 Wochen die verschiedenen Gewebe und das Serum entnommen und analysiert. Die Adipokinspiegel im Serum wurden mittels ELISA gemessen. Die Progression der OA wurde histologisch (H/E-, Safranin-O-, Pappenheim-, und Masson-Goldner-Färbungen) dargestellt und eingestuft. Die Darstellung lokaler Adipokinmuster und eine Klassifizierung der vorhandenen Zelltypen wurden immunhistologisch umgesetzt. Metabolische Parameter wurden mit der OA-Progression korreliert.

Ergebnisse: Die OA Induktion war zu jedem Zeitpunkt signifikant und besonders ausgeprägt unter HFD (z.B. OA Score nach 6 Wochen: HFD 3,7 vs. ND 1,4). Die serologischen Leptinwerte wurden im Vergleich zu ND durch HFD signifikant gesteigert, was auf eine Leptin- und Insulinresistenz schließen lässt. DMM führte zu einer konsistenten Reduktion der Leptinwerte, unabhängig von der Diät (z.B. nach 4 Wochen: HFD Kontrollgruppe 18,4 ng/ml vs. HFD DMM 3,7 ng/ml). Die Ausprägung der Leberverfettung korrelierte mit den serologischen Leptinwerten. Interessanterweise zeigte Adiponektin systemisch nur eine signifikante, OA-induzierte Veränderung zum spätesten Zeitpunkt (HFD Kontrollgruppe $5176 \mathrm{ng} / \mathrm{ml}$ vs. HFD DMM $6149 \mathrm{ng} / \mathrm{ml}$ ). HFD, DMM oder die Kombination beider Modelle zeigte keine signifikanten Einflüsse auf die Expression von Visfatin und IL-6.

Fazit: Die Daten zeigen, wie bereits im Menschen beobachtet, eine verstärkte OA-Progression bei Adipositas. Die Einflüsse des Metabolismus sind u.a. durch höhere Anzahlen adipokinexprimierender Zellen, v.a. lokal im Gelenk,erklärbar. Interessanterweise sind diese lokalen Adipokinexpressionen nicht an die systemischen Adipokinspiegel gekoppelt. 
Immunologie / Infektiologie II

\section{PS072 \\ Dog bite leading to acute cholecystitis and fulminant sepsis complicated by thrombotic microangiopathy and multiorgan failure: a case report}

Esther Leenen; Matthias Kamm; Ana Harth; Achim Jörres

Kliniken der Stadt Köln gGmbH, Köln

Background: Capnocytophaga canimorsus is a facultative anaerobic Gram-negative bacterium which is present in the saliva of dogs and is usually transmitted via bites. It is known to cause potentially fatal blood stream infections and is often associated with bleeding abnormalities, such as petechiae, purpura fulminans, or disseminated intravascular coagulation (DIC). However, forms of clinical presentation can vary immensely. Immunocompromised patients with asplenia or a history of alcohol abuse are considered at highest risk.

Case presentation: A 66-year old man with no previous medical history presented to a nearby hospital with dyspnea, high fever and watery diarrhoea. First Symptoms occurred on the day before. Medical examination showed tachycardia, tachypnea and cyanosis of acra. The abdomen was found to be tender and not painful. Laboratory tests revealed elevated C-reactive protein (CRP), haemolytic anaemia and thrombocytopenia. Coagulopathy, hyperbilirubinaemia, kidney and liver dysfunction could also be detected. Abdominal computed tomography and ultrasonography demonstrated oedematous thickening of the gallbladder-wall without gallstones. Based on the presumptive diagnosis of acute cholecystitis the patient was treated with antibiotics and transferred to our hospital where immediate cholecystectomy was performed. Intraoperatively the gallbladder appeared only mildly inflamed. Subsequently, his clinical status rapidly deteriorated and purpura fulminans occured. Multiple transfusions were required in treatment of severe anaemia and thrombocytopenia. TMA with beginning acral necrosis was diagnosed based on schistocytosis, coombs negative haemolysis and renal dysfunction and was treated with plasmapheresis. Renal replacement therapy was initiated for persistent anuria and antibiotic therapy was continued. Finally, C. canimorsus was isolated from two blood culture sets. Thereupon the history of a previously unmentioned dog bite was uncovered. Following surgical wound excision of the minor skin lesion the patient's condition improved.

Conclusion: Here we present the rare case of a dog bite-related fulminant Capnocytophagus canimorsus-induced sepsis complicated by TMA, haemolytic anaemia and multiorgan failure. Misleading symptoms initially led to cholecystectomy. However, the underlying diagnosis was uncovered and treatment was started albeit with a certain delay. This case report exemplifies the indispensability of obtaining a detailed medical history and performing a thorough clinical examination including the entire body surface in patients presenting with infections of unknown origin. Furthermore we would like to highlight the importance of administration of antibiotics following men-,dogand cat- bites in order to prevent blood stream infections with Capnocytophagus species.
PS073

Symptomatologie und klinischer Verlauf bei hospitalisierten Erwachsenen mit Virusinfektionen durch Influenza A und RSV

Marc Lepiorz ${ }^{1}$; Andreas Ambrosch ${ }^{1}$; Stefan Schroll ${ }^{2}$

1Krankenhaus Barmherzige Brüder Regensburg, Regensburg

Einleitung: Zum klinischen Profil und Verlauf von komplizierten Infektionen mit RSV bei Erwachsenen. Im Vergleich zur Influenza A ist wenig bekannt. Die vorliegende Untersuchung hatte deshalb zum Ziel prädisponierender Grunderkrankungen, klinischem Profil, Verlauf und Prognose bei hospitalisierten. Patienten mit RSV-, und Influenza A-Infektionen miteinander zu verglichen.

Material und Methode: Eingeschlossen wurden Patienten mit RSV- und Influenza A Infektionen, die zwischen 1.1.2017 und 31.3.2017 hospitalisiert wurden. Zur Charakterisierung wurden klinische Symptome, Laborparameter sowie prädisponierende chronische Grunderkrankungen erfasst. Darüber hinaus wurde Verlauf und Schwere der Infektion anhand verschiedener klinischer Parameter einschließlich Beatmungspflichtigkeit und Mortalität abgeschätzt.

Ergebnisse: Insgesamt wurden 191 Patienten mit Influenza A und 98 Patienten mit RSV-Infektion ausgewertet. Sowohl das mittlere Alter als auch die klinische Symptomatologie bei Diagnosestellung waren in beiden Patientengruppen vergleichbar. Nur 2/3 der Patienten zeigten Influenza-typische respiratorische Symptome. Zwischen 16 und $17 \%$ der Infektionen waren nosokomialen Ursprungs. RSV-Patienten litten fast doppelt so häufig an chronischen Nierenerkrankungen wie Patienten mit Influenza A (35\% vs. $18 \%$ bei Influenza, p < 0.001). Bezüglich klinischem Verlauf mussten Patienten mit RSV-Infektion häufiger invasiv beatmet werden ( $10 \%$ vs. $3.6 \% ; p=0.05$ ) und zeigten eine fast doppelt so hohe Mortalität ( $14 \%$ vs. $7 \%, p=0.049$ ).

Schlussfolgerung: RSV-Infektionen kommen als häufige Ursache von komplizierten Influenzaähnlichen Erkrankungen bei älteren, hospitalisierten Patienten in Frage. Im Vergleich zur Influenza A verlaufen RSV-Infektionen jedoch schwerer. Bei beiden Entitäten ist ein nicht unerheblicher Teil der Infektionen nosokomialen Ursprungs ist, was im Hinblick auf ein erfolgreiches Hygienemanagement eine möglichst rasche Diagnosestellung impliziert.

\section{PS074}

The incretin hormone GLP-2 is secreted from pancreatic alpha cells during acute inflammation through IL- 6 and protects against polymicrobial sepsis

Robert Werner Mertens'; Florian Kahles'; Sebastian Diebold'; Julia Möllmann; Irmgard Diepolder'; Benedikt Walla'; Elias Haj-Yehia'; Darleen Sandoval ${ }^{2}$; Ann Christina Foldenauer'; Lukas Martin'; Tobias Schürholz ${ }^{3}$; Jan Brünsing'; Alexander Koch'; Frank Tacke'; Corinna Lebherz'; Nikolaus Marx'; Michael Lehrke

${ }^{1}$ Uniklinik RWTH Aachen; ${ }^{2}$ University of Michigan; ${ }^{3}$ Universitätsmedizin Rostock

Background: The incretin GLP-2 (glucagon-like peptide-2) is secreted by intestinal L-cells following food intake, enhances intestinal nutrient absorption and is clinically used for the treatment of patients with short bowel syndrome. Recently, GLP-2 has been found to be upregulated and to be protective in patients with colitis through as yet unknown mechanisms. Here we sought to characterize the role of GLP-2 during systemic inflammation. 
Methods and Results: To analyze whether the GLP-2 system is modulated by the immune system, we measured circulating GLP-2 levels in patients under inflammatory conditions. 223 critically ill patients admitted to the ICU showed a 3.9-fold increase of total GLP-2 plasma levels in comparison to 53 healthy controls $\left(3.0 \mathrm{ng} / \mathrm{mL}\right.$ vs. $11.4 \mathrm{ng} / \mathrm{mL}_{\text {; }}$ $\mathrm{p}<0.001)$. Among the ICU cohort higher GLP-2 concentrations were found in patients presenting with sepsis and more severe disease. Moreover, GLP-2 levels were significantly correlated with markers of inflammation (IL-6, PCT, CRP) and independently predicted 30 day ICU and overall mortality in critically ill patients. To examine whether GLP-2 secretion is directly regulated by inflammation we injected C57BL/6 mice with LPS (i.p.). LPS ( $1 \mathrm{mg} / \mathrm{kg}$ ) increased GLP-2 secretion with a max. of $17.2 \pm 4$-fold $(p<0.01)$ after 210 minutes. Further experiments in IL1R-/- and IL6-/- mice showed that LPS-induced GLP-2 secretion is mediated by IL- 6 . To identify the source of GLP-2 secretion in settings of inflammation, we injected LPS into transgenic mice with tissue-specific reactivation of the preproglucagon gene (Gcg) in the gut (GcgRA $\triangle V i l C r e)$ or the pancreatic cell (GcgRA $\left.\triangle P D X_{1}-C r e\right)$. Interestingly, LPS-induced GLP-2 secretion was blocked in GcgRA $\triangle$ VilCre mice, while GcgRA $\triangle P X_{1}-C_{1}$ mice showed a marked increase of GLP-2, indicating that inflammation-dependent GLP-2 production is derived from the pancreas and not as expected from the gut. Finally, we wondered whether inflammatory upregulation of GLP-2 has functional relevance and administered GLP-2 (1-33) or saline as control per central vein catheter mice with sepsis who underwent cecal ligation puncture. GLP-2 protected against polymicrobial sepsis by improving hemodynamic parameters and clinical sepsis score. Mechanistically GLP-2 decreased sepsis-induced elevation of TNF- $a$, IL- 6 and IL-1 $\beta$ serum levels and blocked proinflammatory cytokine expression in various tissues.

Conclusion: GLP-2 secretion is directly activated by IL-6 in systemic inflammation in mice and humans independent of food intake. Surprisingly, under systemic inflammation GLP-2 is derived from the pancreas and not from the gut as we would expect after nutrient intake. Functionally, GLP-2 protects mice against polymicrobial sepsis by blocking inflammatory signalling. GLP-2 as an endogenous counterregulatory peptide provides a previously unknown link between the pancreas and the immune system, which might open new avenues for the treatment of patients with sepsis.

\section{PS075 \\ Infliximab als Salvage-Therapie bei 60 -jähriger Patientin mit Leberzirrhose Child B bei therapierefraktärer Autoimmunhepa- titis Typ I}

Clara Nusser; Bernhard Michels; Rebecca Mayr; Stephan Schmid; Michael Selgrad; Martina Müller-Schilling

Uniklinikum Regensburg, Regensburg

Hintergrund: Die Autoimmunhepatitis ist eine seltene, akute, chronisch-entzündliche Autoimmunkrankheit der Leber, welche unbehandelt zur Leberzirrhose und ihren Komplikationen führt. Die Standardtherapie mit Azathioprin und Prednisolon erzielt bei den meisten Patienten eine Remission. Für Patienten jedoch, welche auf die Standardtherapie nicht ansprechen oder sie nicht vertragen, gibt es nur wenige Alternativen mit teilweise unzureichenden Therapieerfolgen.

Case Report: 2006 stellte sich die damals 49-jährige Patientin mit einer unklaren Erhöhung der Leberwerte vor. Es wurde die Erstdiagnose einer Autoimmunhepatitis gestellt und nach initialer Stoßtherapie mit Prednisolon leitliniengerecht eine Therapie mit Azathioprin begonnen. Bei Haarausfall, massiver Abgeschlagenheit und Panzytopenie erfolgte im März 2016 die Umstellung der Therapie auf Mycophenolatmofetil. In der Abdomen Sonographie zeigte sich zu der Zeit zum ersten Mal ein zirrhotischer Umbau der Leber. Im August 2016 kam es zum Relapse der Autoimmunhepatitis und Einleitung einer Cortisonstoßtherapie, die mit Prednisolon und Budesonid fortgeführt wurde. Bei ausgeprägter Leukozytopenie erfolgte im März 2017 die Umstellung auf eine Therapie mit Tacrolimus, welche bei Panzytopenie und Kreatininanstieg abgebrochen werde musste. Daraufhin erfolgte die off-lable Umstellung auf Infliximab als Salvage-Therapie. Zeitgleich erfolgte die Aufnahme auf die Lebertransplantationsliste. Es erfolgten insgesamt 4 Gaben von Infliximab (Woche o, Woche 2, Woche 6, Woche 10). Im Verlauf kam es zur raschen Normalisierung der Leberwerte. Die Therapie konnte ohne relevante Nebenwirkungen appliziert werden. Nach der 4. Gabe trat allerdings eine schwere Neutropenie auf. Diese konnte nach Gabe von GCSF rasch kontrolliert werden. Seit der letzten Gabe von Infliximab im Juni 2017 befindet sich die Patientin unter keiner immunsuppressiven Therapie. GOT, GPT, Gamma-GT und AP liegen seit Juli 2017 über den Zeitraum von 4 Monaten im Normbereich. Das Blutbild ist hinsichtlich der Leukopenie nur noch geringgradig eingeschränkt. Die Patientin selbst berichtet über eine Steigerung der Lebensqualität. Relevante Infektionen traten während des Behandlungszeitraums nicht auf.

Schlussfolgerung: Der vorliegende Case Report zeigt einen schweren, therapierefraktären Verlauf einer Autoimmunhepatitis. In Anbetracht der Tatsache, dass im vorliegenden Fall bereits zahlreiche Therapieversuche mit nicht tolerablen Nebenwirkungen unter der immunsuppressiven Therapie vorausgingen und zu Therapiebeginn bereits eine Leberzirrhose Child B vorlag, zeigte sich ein hervorragendes klinisches und laborchemisches Ansprechen auf die Therapie mit Infliximab. Es gelang eine fortlaufende Kontrolle der Erkrankung mit erheblicher Steigerung der Lebensqualität. Die Therapie mit Infliximab kann von daher als Salvage-Therapie bei einer schwer zu behandelnden Autoimmunhepatitis in Betracht gezogen werden.

\section{PS077}

Successful treatment of refractory severe acute humoral rejection in a renal transplant with double filtration plasmapheresis plus immunoadsorption

Martin Rußwurm; Tanja Maier-Giebing; Joachim Hoyer

Universitätsklinikum Marburg, Marburg an der Lahn

Introduction: Acute humoral rejection (AMR) is a major threat to renal allograft survival in the early transplant period, it accounts for up to $40 \%$ of graft losses within the first year. Although various immunomodulatory treatments $(\mathrm{Tx})$ are used to treat AMR, there is no golden standard. In every unique clinical situation regarding Mismatch, serological follow-up, severity of AMR, long- and short-term adverse events of immunosuppressive Tx, careful consideration which $\mathrm{Tx}$ to choose is imperative. As far as there is still a lack of understanding not only pathomechanisms but also how to integrate the ample histological and immunological data available in the single case, rational for Tx still seems to be somewhat arbitrary and is subject to the treating physicians experience. Nevertheless, there are situations in which one might leave the beaten track. Here we present a case of severe, sustained early AMR, treated with the various immunosuppressive agents and techniques in the armamentarium, finally AMR being successfully resolved by an extensive plasmapheresis approach.

Case report: At renal transplantation, immunosuppressive Tx has been initiated with Cyclosporin, Methylprednisone (Predni) and Mycophenolate. While the Pt. developed no graft function postoperatively, she underwent the first renal transplant biopsy $(\mathrm{Bx})$ at $\mathrm{d} 6$ followed by a pre-emptive Predni pulse for Tx of suspected acute cellular rejection. The first histology report, however, revealed the typical picture of AMR. We then initiated Tx for AMR with a course of anti-thymocyte 
immunoglobulin, Plasma Exchange, Rituximab, Immunoadsorption (IA) and intravenous immunoglobulin, the Pt. remaining anuric still. We decided to serial-connect a high-molecular weight filter device, called double filtration plasmapheresis (DFPP) with an IA system, treating the pt. every other day. After the $2 n d$ Tx the transplant developed excretory function, accompanied by significant decline in serum creatinine. Follow-up Bx at day 50 still showed active AMR but with significantly lesser activity. The pt. was dismissed at d60 with improved and stable renal function. We performed DFPP+IA thrice weekly for 2 months and consecutively weaned the patient over one year. The last SCr was $1,52 \mathrm{mg} / \mathrm{dl}$.

Discussion: The capability of DFPP+IA in clearing main effectors of AMR was the rational to adopt this approach in an experimental Tx intent. Although we cannot rule out the impact of long-term effects of the preliminary treatments, the chronological relationship of DFPP+IA initiation and renal recovery appears convincing. To our knowledge, this is the first report of DFPP+IA for Tx of severe AMR. Thus, we consider the implications of this case to be twofold: First, aggressive treatment of severe AMR could be successful even in long-term graft dysfunction. Second, DFPP+IA should be considered a rescue-treatment option for selected high-risk patients with proven severe acute humoral rejection in which other treatments failed.

\section{PS078 \\ IRF3 und IRF7 sind zentrale Elemente der Typ I IFN Antwort bei Hepatitis B}

David Schwarz'; Yuri Churin'; Hans-Joachim Mollenkopf ${ }^{2}$; Martin Roderfeld'; Elke Roeb ${ }^{1}$

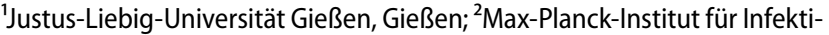
onsbiologie, Berlin

Einleitung: Die Therapie der chronischen Hepatitis B mit Interferon-alpha zeigt nur bei einem Bruchteil der Behandlungen eine klinische Verbesserungen. In Genexpressionanalysen konnte dabei gezeigte werden, dass bei Therapieversagen eine Assoziation mit einer prätherapeutisch latenten Aktivierung von Interferon-stimulierten Genen (ISGs) vorliegt. Diese werden unter anderem durch die Interferon-regulierenden Faktoren 3 und 7 (IRF3, IRF7) reguliert. Die beiden Transkriptionsfaktoren sind zentrale Elemente der Modulation der Typ I Interferon (IFN) Antwort. Zur Überprüfung ihrer Funktion in der Immunantwort im Rahmen einer chronischen Hepatitis B wurde von uns ein Mausmodell etabliert, das einen IRF3/7 Doppelknockout mit HBsAg Transgenität kombiniert.

Methoden: Ein IRF3/7 Doppelknockout (KO) wurde mit einer HBsTg Balb/c (TG) Maus gekreuzt und eine dreifach transgene Mauslinie etabliert. An 12 Wochen alten Mäusen wurde der Einfluss des IRF3/7 Doppelknockouts auf die chronische Lebererkrankung in HBsAg transgenen Mäusen untersucht. Die vergleichende Analyse wurde mittels klinischer Laborparameter, Microarray, sowie qRT-PCR, Proteom Profiler, Western-Blot und Histologie durchgeführt.

Ergebnisse: Im Microarray zeigten TG-KO Mäuse eine Inhibition der ISGs, was in QPCR und im Western Blot bestätigt wurde. Eine Analyse von Serum-Zytokinen wies gleichzeitig teilweise deutliche Herunterregulationen bedingt durch den $\mathrm{KO}$ auf. Lebertransaminasen und Pathohistologie zeigten zum Untersuchungszeitpunkt in der vergleichenden Überprüfung der Mauslinien keinen signifikanten Unterschied.

Schlussfolgerung: Mit der vorliegenden Studie konnte gezeigt werden, dass IRF3 und IRF7 auch im Rahmen der HBV Erkrankung zentrales Element der Typ I IFN Antwort sind. Während das Ausmaß der Lebe- rentzündung nicht durch den KO beeinflusst wurde, waren Zytokine und vor allem ISGs herunterreguliert. Der etablierte TG-KO ermöglicht die weitere in vivo Untersuchung und gegebenenfalls Aussagen darüber, inwieweit die Antagonisierung der beiden Transkriptionsfaktoren oder assoziierter ISGs therapeutischer Ansatzpunkt für eine Erhöhung der Erfolgsrate einer Interferon-alpha Therapie sein könnte.

\section{PS079 \\ Dermatomyositis or immune-mediated necrotizing myopathy - an interesting case report}

Sophie Teege; Friedrich Haag

Universitätsklinikum Hamburg -Eppendorf, Hamburg

We present a rare case of immune-mediated necrotizing myopathy (IMNM) in a 38-year old male patient with no statin intake. The patient first presented in October 2016 with muscle weakness and cutaneous lesions on his fingers, which were interpreted as Gottron's papules in an external hospital in Hamburg. The muscle biopsy was compatible with the diagnosis of dermatomyositis, although with atypical features. Although the patient was negative for a standard panel of myositis-specific autoantibodies, i.e. SRP, Mi2, PL-7, PL-12, Ku, PM-Scl75, EJ, OJ, Ro-52, and PM-Scl10o, tested as a line immunoassay in our immunology laboratory, the clinical diagnosis of dermatomyositis was established.

In July 2017, the symptoms worsened, and the patient was admitted to the University Hospital of Hamburg as an inpatient. In order to establish a final diagnosis, the serum was sent to the Laboratory for Immunology and Biotherapy in Rouen, France, to test for further known antibodies associated with myopathies, specifically MDA-5, TIF-1 gamma, NXP-2, SAE and antibodies against HMGCR. Surprisingly, the antibodies for HMGCR (3-hydroxy-3-methylglutaryl-coenzyme A reductase) were strongly positive $(105,1 \mathrm{UA} / \mathrm{ml}$, referenc e value $<20$ / $\mathrm{UA} / \mathrm{ml}$ ). This antibody is specific for immune mediated necrotizing myopathy (IMNM). Although this disease is usually associated with statin intake, the patient had no history of statin intake. The original biopsy specimen from October 2016 was re-evaluated, and found to be equally compatible with the suspected diagnosis of IMNM, albeit with some features characteristic of dermatomyositis, such as perifascicular atrophy type 1 and 2 , as well as some features that can be found in both dermatomyositis and IMNM, such as focal necrosis. Treatment with $2 \times 1000 \mathrm{mg}$ Rituximab had already been initiated after treatment efforts with azathioprine and cyclophosphamide, as well as IVIG had been unsuccessful. The patient is currently in his follow-up phase, and treatment with Rituximab has led to an immense improvement in his clinical symptoms.

In conclusion, the detection of an autoantibody was the only reliable method to make a diagnosis in this patient as the cutaneous symptoms were misleading and the muscle biopsy was not specific enough for either dermatomyositis or IMNM.

\section{PS080}

\section{Kasuistik: NSTEMI und erhöhte Inflammationsmarker}

Ildiko Toth ${ }^{1}$; Astrid Hummel ${ }^{2}$; Silvia Ribback ${ }^{3}$; Frank Dombrowski ${ }^{3}$; Piotr Kaczmarek $^{2}$; Stephan Felix ${ }^{2}$; Lerch Markus ${ }^{4}$; Lukas Bossaller ${ }^{1}$ 
'Universitätsmedizin Greifswald, Klinik Innere Medizin A, Bereich Rheumatologie, Greifswald; ${ }^{2}$ Universitätsmedizin Greifswald, Klinik für Innere Medizin B, Abteilung Kardiologie, Greifswald; ${ }^{3}$ Universitätsmedizin Greifswald, Institut für Pathologie, Greifswald; ${ }^{3}$ Universitätsmedizin Greifswald, Institut für Pathologie, Greifswald; ${ }^{2}$ Universitätsmedizin Greifswald, Klinik für Innere Medizin B, Abteilung Kardiologie, Greifswald; ${ }^{2}$ Universitätsmedizin Greifswald, Klinik für Innere Medizin B, Abteilung Kardiologie, Greifswald; ${ }^{4}$ Universitätsmedizin Greifswald, Klinik Innere Medizin A, Greifswald; ' Universitätsmedizin Greifswald, Klinik Innere Medizin A, Bereich Rheumatologie, Greifswald

Hintergrund: Die Riesenzellarteriitis (RZA) ist die häufigste systemische Vaskulitis im späteren Erwachsenenalter. Die klinische Symptomatik der Arteriitis temporalis reicht von den typischen temporalen Cephalgien mit palpablen und verdickten Arteriae temporales, proximal-betonten Myalgien sowie einer Kau-Claudikatio bis zu der gefürchteten Erblindung in Folge einer Anterioren Ischämischen Optikusneuropathie. Raritäten sind intrakranielle vaskulitische Manifestationen oder Koronariitiden.

Methode: Wir berichten von einer 77 jährigen Patientin, welche über die Notaufnahme mit einer progredienten Belastungsdyspnoe ohne typische AP-Symptomatik sowie erhöhten Entzündungswerten und Myalgien vorstellig wurde. Bei Aufnahme waren das Troponin I (cTnl) i.PI. mit $0.65 \mu \mathrm{g} / \mathrm{l}$, das C-reaktives Protein (CRP) mit 132mg/l, das Ferritin mit $507 \mu \mathrm{g} / \mathrm{l}$ und die BSG mit $82 \mathrm{~mm} / \mathrm{h}$ erhöht. Bei V.a. nichttransmuralen Myokardinfarkt (NSTEMI) erfolgte eine Koronarangiographie, bei der sich neben einer 20\% Lumeneinengung im Ramus interventricularis anterior zusätzlich eine $80 \%$ Abgangstenose des ersten Diagonalastes fand, die mittels PTCA und Cutting-Balloon interveniert wurde. Aufgrund des erhöhten CRPs wurde eine Endokarditis mittels Blutkulturen, TTE und TEE ausgeschlossen. Nach Einleitung einer empirischen Antibiose erfolgte eine rheumatologische Vorstellung. Für eine RZA diagnoseweisend waren hierbei temporale und nuchale Cephalgien, eine Kau-Claudikatio sowie beidseits schmerzhaft tastbare Aa. temporales. Proximale Myalgien bestanden bereits seit ca. acht Monaten vor dem NSTEMI. Intermittierende Prednisolongaben durch den Hausarzt hätten zu einer Rückbildung der proximal-betonten Myalgien geführt und waren jeweils bei Prednisolondosierungen $<7.5 \mathrm{mg} /$ Tag rezidiviert. An kardiovaskulären Risikofaktoren lagen lediglich ein arterieller Hypertonus und eine Hyperlipidämie vor. Das retrospektiv errechnete 10-Jahres Risiko für atherosklerotische kardiovaskuläre Ereignisse, berechnet nach den 2012 ESC Guidelines, war mit 3\% nur gering erhöht. Eine Makroangiopathie der Aorta, der Aa. Carotides oder Aa. Vertebrales war sonographisch nicht nachweisbar. Die Myalgien, die Kau-Claudikatio, die temporalen Cephalgien und die Dyspnoe sistierten nach Einleitung einer Therapie mit Prednisolon 10omg/Tag und laborchemisch normalisierten sich CRP und CTnl im weiteren Verlauf.

Ergebnis: Aufgrund der klinischen Präsentation, der Serologie und der typischen Histologie der Biopsie der A. temporalis stellten wir die Diagnose einer Riesenzellenarteriitis. Bei nur gering erhöhtem kardiovaskulären Risiko liegt nahe, dass eine Koronariitis bei Riesenzellarteritis das akute Koronarsyndrom möglicherweise begünstigt hat. Die vaskulitische Koronariitis im Rahmen einer Riesenzellenarteriitis ist eine seltene, aber wichtige Differentialdiagnose bei akutem Koronarsyndrom.
PS081

\section{Morbus Whipple: ein Reprospektive Fallserie über 13 Jahre}

Ulrike von Arnim; Armin Hoffmann; Peter Malfertheiner; Ali Canbay

Universitätsklinikum Magdeburg A.ö.R., Magdeburg

Einleitung: Morbus Whipple (MW) stellt eine sehr seltene multisystemische chronische Infektionserkrankung dar, unbehandelt kann sie tödlich verlaufen. Hauptsymptome sind Gewichtsverlust, Arthralgien und chronische Diarrhoen. Häufig bestehen rheumatische Beschwerden jahrelang vor dem Auftreten von gastrointestinalen Symptomen und werden oft als seronegative Polyarthritis fehlgedeutet sowie immunsuppressiv behandelt.

Ziel: klinische Charakterisierung der MW- Fälle in einer retrospektiven Fallserie von 2002 bis 2015.

Ergebnisse: von 2002-2015 wurden 8 Patienten (Pat.) mit einem MW diagnostiziert. $7 / 8$ Pat. (87,5\%) berichteten über Arthralgien, welche durchschnittlich 6,5 Jahre (78 Monate, Spanne 8 Monate bis 10 Jahre) vor der Erstdiagnose des MW bereits bestanden. Bei 6/8 (85\%) Pat wurde eine seronegative rheumatoide Arthritis diagnostiziert und in allen Fällen immunsuppressiv mit Prednisolon $(4 / 6,67 \%)$, Methotrexat $(3 / 6,50 \%)$ und/oder Biolgika $(1 / 6,17 \%)$ therapiert. Weitere häufige klinische Befunde waren: Anämie (4/8,50\%), Malabsorption (3/8, 37.5\%) und Gewichtsverlust (4/8,50\%). Als extraintestinale Manifestationsorgane konnten Herz(3/8) Lunge (3/8) und zentrales Nervensystem (3/8) gesichert werden.

Schlussfolgerung: Die Mehrzahl derMW- Erkrankten unserer Kohorte wiesen als Initialsymptom Arthralgien Jahre vor Erstdiagnose des MW auf. Bei therapierefraktären arthralgischen Beschwerden sollte früher an das Vorliegen eines MW gedacht werden.

\section{Innere Medizin integral}

\section{PS082}

Seltener Fall einer 65-jährigen Patientin mit neu diagnostizierter adulter Riesenzellhepatitis und synchroner Coombs-positiver autoimmunhämolytischer Anämie

Andreas Albert; Christian Forster; Kilian Weigand; Martina Müller-Schilling; Georg Peschel

Universitätsklinikum Regensburg, Regensburg

Wir berichten von einer 65-jährigen Patientin, welche uns mit dem klinischen Bild einer Sepsis und einer unklaren Leberraumforderung in der Sonographie zu verlegt wurde. Bereits 11/2015 waren erhöhte Leberwerte aufgefallen. Histologisch fand sich damals a.e. das Bild einer medikamentös-toxischen Hepatitis. Bei der Patientin ist seit 06/2016 zudem eine Coombs-positive autoimmunhämolytische Anämie vom Typ IgG mit $\mathrm{C}_{3} \mathrm{~d}$-Beladung der Patientenerythrozyten bekannt. Diese war mit Cortison und Azathioprin behandelt. Darunter bestand bis zwei Wochen vor Aufnahme klinisches Wohlbefinden. Die Fokussuche bei uns ergab den Nachweis einer Urospesis mit E. faecium und Candida albicans. Gleichzeitig bestanden ein akutes Leberversagen und eine hämolytische Anämie (GOT 639 U/l; GPT 734 U/l; Gamma-GT $115 \mathrm{U} / \mathrm{l}$; Alk. Phosphatase $178 \mathrm{U} / \mathrm{l}$; Bilirubin (ges.) $24.8 \mathrm{mg} / \mathrm{dl}$; Albumin $19.9 \mathrm{~g} / \mathrm{l} ;$ CRP $85.9 \mathrm{mg} /$; Haptoglobin 


\section{PS083}

Endoskopische transmurale Vollwandresektion (EFTR) am unteren Gastrointestinaltrakt (GIT): Welche Patienten profitieren?

Heinz Albrecht'; Martin Raithel ${ }^{2}$; Andreas Stegmaier ${ }^{3}$; Andrea Nagel2 ${ }^{2}$; Axel Braun $^{2}$; Claus Schäfer ${ }^{1}$

'Kliniken des Landkreises Neumarkt i.d.OPf., Neumarkt; ${ }^{2}$ Malteser Waldkrankenhaus St. Marien Erlangen, Erlangen; ${ }^{3}$ Stadtkrankenhaus Schwabach, Schwabach;

Hintergrund: Die endoskopische transmurale Vollwandresektion (EFTR) erweitert das endoskopische Resektionsspektrum, indem diese Methode die Resektion von Läsionen erlaubt, die keine Abhebung von der Mukosa zeigen, die submukös liegen oder bereits ein Mukosakarzinom beinhalten.

Methoden: Ziel dieser Auswertung ist die Analyse der zugewiesenen Patienten für eine EFTR, die gezielte Histologie der abgetragenen Läsion sowie die Darstellung der sich daraus ergebenden klinischen Ergebnisse und ihre Auswirkungen auf den Verlauf des Patienten. Die EFTR wurde entsprechend der zugelassenen Standardindikation mit dem Vollwandresektionsset (FTRD) der Fa. Ovesco (Tübingen) in Analgosedierung durchgeführt. Insgesamt wurden im Zeitraum Nov. 2014 bis Feb. 2017 an 3 Kliniken mit Schwerpunktendoskopie 55 Patienten mit potentieller EFTR-Indikation identifiziert.

Ergebnisse: Zwei der initial für geeignet gehaltenen Läsionen stellten sich endoskopisch als zu groß dar und wurden daher primär operiert Vier Läsionen wurden aufgrund der Lage oder der fehlenden Auffindbarkeit nicht reseziert. Von den übrigen 49 Läsionen handelte es sich um 21 Rezidivadenome und/oder Läsionen mit fehlender Abhebbarkeit, 21 Läsionen mit high-grade intraepithelialer Neoplasie und/oder intramukosalem Adenokarzinom, 6 submukösen Läsionen/NETs und eine Metastase eines malignen Melanoms. Von allen Läsionen (Größe $2,5+/-2 \mathrm{~cm})$ konnten mittels EFTR 38 (77,6\%) komplett reseziert werden (Ro), 11 Läsionen (22,4\%) waren inkomplett (R1 bzw. R2). Bezogen auf alle Fälle war aufgrund einer inkompletten Abtragung in acht Fällen eine chirurgische Nachoperation notwendig (8/49; 16,3\%). Zudem musste bei drei Patienten nachoperiert werden, da das Vollwandresektat einen frühzeitigen Befund einer Lymphgefäßinfiltration ergab $(3 / 49 ; 6,1 \%)$.

Es traten folgende Major- und Minorkomplikationen $(n=4)$ auf: einmal kam es zu einer $\mathrm{Hb}$-relevanten Blutung, die endoskopisch beherrscht werden konnte, einmal musste die Abtragung abgebrochen werden, da es nicht gelang ausreichend Gewebe in die Kappe zu ziehen, einmal kam es zu einer Perforation im Sigma, die ohne OP mittels OTSC (Ovesco, Tübingen) versorgt werden konnte und einmal erfolgte nur eine inkomplette Abtragung, da die Schlinge und der Grasper bei der Abtragung dislozierten.

Schlussfolgerung: Zusammenfassend zeigen diese ersten Daten, dass bei fehlender Abhebbarkeit (non-lifting sign), fortgeschrittener Histologie von Colonadenomen und anderen Indikationen wie z.B. submuköse Raumforderungen bei mehr als zwei Drittel der eingeschlossenen Patienten eine chirurgische Operation durch die Möglichkeit der EFTR vermieden werden kann. Exemplarisch ist bei allen NETs durch die Methode eine definitive Therapie erreicht worden. Andererseits sollte die Indikation zu EFTR bei größeren Läsionen ( $>4$ $\mathrm{cm}$ ) und beim Nachweis von Mukosakarzinomen in der Biopsie streng gestellt werden, da diese Patienten ggf. von einem primär chirurgischen Eingriff mehr profitieren können.
PS084

Wirksamkeit der Sensors Augmented Pump Therapie mit prädiktiver Insulinsuspension bei Patienten mit Diabetes mellitus Typ 1

Triantafyllos Didaggelos'; Parthena Giannoulaki'; Anastasia Paschala'; Euaggelia Kotzakioulafi ${ }^{2}$; Eleni Karlafti'; Zisis Kontoninas ${ }^{3}$; Anna Mavromanoli'; Apostolos Chatzitolios'

${ }^{1}$ University General Hospital of Thessaloniki AHEPA, Thessaloniki; ${ }^{2}$ Privat praxis, Thessaloniki, Greece; ${ }^{3}$ Privatärztliche Praxis, Veroia, Greece

Zweck: Untersuchung der Wirkung auf die metabolische Kontrolle der Blutzuckers vor und nach der sensorgesteuerten Pumpentherapie mit prädiktiver Insulinsuspensionstechnologie bei Patienten mit Diabetes Mellitus Typ 1, die zuvor mit anderen Pumpentypen oder MDI behandelt worden sind. Eine sensorgestützte Insulinpumpe unter Verwendung des MiniMed 640G Systems mit Smart-Guard-Technologie ermöglicht eine automatischen Insulinabgabe basierend auf der Vorhersage eines niedrigen Blutzuckerspiegels.

Patienten-Methoden: Es wurden 13 Patienten eingeschlossen mit Typ-1-Diabetes, mit mindestens drei Monaten Behandlung mit dem MiniMed 640G System SmartGuard- Technologie (Medtronic), davon 7 Frauen, Alter $44,7 \pm 12,4$ Jahre, $\mathrm{BMI}=23,7 \pm 4,2 \mathrm{~kg} / \mathrm{m} 2$, mittlere Dauer des Diabetes mellitus $=27,7 \pm 7,8$ Jahre). $\mathrm{HbA}_{1} \mathrm{C}$, anthropometrische Messungen und medizinische Anamnese wurden vor und nach der Verwendung des MiniMed 640G Systems aufgezeichnet. Zusätzlich wurden die Daten der Insulinpumpe des letzten Monats mit der Software Careling Pro und Personal Medronic aufgezeichnet.

Schlussfolgerung: Die glykämische Kontrolle nach der Verwendung des $640 \mathrm{G}-$ Systems war signifikant besser als vor der Verwendung(Mittelwert $\mathrm{HbA}_{1} \mathrm{C}=6,54 \pm 0,4$ gegenüber 7,36 $\pm 0,8, \mathrm{p}=0,002$ ). Der mittlere glykämische Variabilitätsprozentsatz zwischen $70-180 \mathrm{mg}$ / dl betrug $75,8 \%$ und über $180 \mathrm{mg} / \mathrm{dl}$ und weniger als $70 \mathrm{mg} / \mathrm{dl}$ betrug 21,09\% und 3,1. Es gab einen Unterschied der Gesamttagesdosis (Tagesgesamtdosis, TDD) vor und nach der Verwendung des Systems (Durchschnitt TDD $=43,2 \pm 20,1$ gegenüber 37,1 $\pm 17,9, p=$ $0,051)$. Schließlich gab es keine signifikante Veränderung des Körpergewichts $(72,9 \pm 19,6$ vs. $70,3 \pm 18, p=0,071)$.

Schlussfolgerung: Bei Patienten mit DM1 optimiert der Einsatz einer sensorgestützten Pumpentherapie mit prädiktiver Insulinsuspensionstechnologie die Blutzuckereinstellung, der Patient verbringt den größten Teil des Tages im Glukosezielbereich ohne Gewichtszunahme.

\section{PS085}

Das Ergebnis der zweijährigen Administration von ACE-Hemmern in der diabetischen peripheren und autonomen Neuropathie bei Patienten mit Diabetes mellitus

Triantafyllos Didaggelos'; Konstantinos Tziomalos'; Anastasia Paschala'; Charalampos Margaritidis ${ }^{2}$; Zisis Kontoninas ${ }^{2}$; loannis Stergiou ${ }^{3}$; Stefanos Tsotoulidis ${ }^{4}$; Anna Mavromanoli; ${ }^{1}$ Apostolos Chatzitolios ${ }^{1}$

'University General Hospital of Thessaloniki AHEPA, Thessaloniki, Greece; ${ }^{2}$ Privatärztliche Praxis, Serres; ${ }^{2}$ Privatärztliche Praxis, Veroia; ${ }^{3}$ General Hospital of Thessaloniki G.Gennimatas, Thessaloniki, Greece; ${ }^{4}$ General Hospital of Chalkidiki, Chalkidiki, Greece

Zweck: Die vorliegende prospektive offene parallele und placebokontrollierte Studie wurde durchgeführt, um die Wirkung von Angiotensin-Converting-Enzym-Inhibitor Quinapril (20 mg / Tag) auf bestimmte diabetische autonome Neuropathie (DAN) mittels Test 
kardiovaskulär Reflexe und peripherer Neuropathie über einen Zeitraum von 24 Monaten festzustellen.

Patienten-Methoden: von 63 Patienten waren 27 Typ-1-Diabetiker und 34 Frauen mit einem mittleren Alter 52 Jahren (22-65). Die Patienten hatten unten klaren diabetischen autonomen ( 2 der folgenden Tests waren abnormal) und periphere Neuropathie gelitten. Die Patienten wurden in 2 Gruppen randomisiert: Gruppe A = 31 Patienten, die Placebo erhielten und Gruppe $B=32$ Patienten, die Quinapril erhielten. Alle Patienten leiden nicht unter Bluthochdruck oder koronare Herzkrankheit (basierend auf normaler Szintigraphie). Wir verwenden die folgenden Methoden zum Nachweis von DPN und DAN: Michigan Neuropathy Screening-Instrumente Fragebogen und Examination (MNSIQ und MNSIE), die Messung der unteren Schwingungswahrnehmungsschwelle mit Biothesiometer (BIO) und CRT [RR-Varianten während tiefer Atmung (bewertet mit Ausatmung / Einatmung Verhältnis (E / I), MCR, SD), Valsalva Maneuver (vals), das Verhältnis 30:15, orthostatische Hypotonie-OH].

Ergebnisse: Die folgenden Ereignisse waren signifikant in der Gruppe A (initial gegen Ende) erhöht: $E / I 1,11 \pm 0,06$ vs 1,23 $\pm 0,12, M C R ~ 18,1 \pm$ 6,2 gegenüber $38,7 \pm 20,5$, SD $31,1 \pm 11,9$ vs. $56,6 \pm 23,0$ (alle $p$

\section{PS086 \\ Single inhaler triple therapy in patients with advanced COPD (FULFIL): exacerbation/pneumonia composite outcomes}

Mark Dransfield'; David Halpin ${ }^{2}$; Helen Barnacle ${ }^{3}$; Cordula Mohrlang ${ }^{3}$; Ruby Birk $^{3}$; Noushin Brealey ${ }^{3}$; Chan-Qing Zhu ${ }^{3}$; David Lipson ${ }^{3}$

'University of Alabama at Birmingham, Birmingham, United States; ${ }^{2}$ Royal Devon and Exeter Hospital, Exeter; ${ }^{3}$ Glaxo Smith Kline, Uxbridge, United Kingdom; ${ }^{3}$ Glaxo Smith Kline, München

Background: FULFIL reported statistically significant improvements in lung function and health status with once-daily fluticasone furoate/umeclidinium/vilanterol (FF/UMEC/VI) $100 \mu \mathrm{g} / 62.5 \mu \mathrm{g} / 25 \mu \mathrm{g}$ via the ELLIPTA $^{\oplus}$ inhaler vs twice-daily budesonide/formoterol (BUD/FOR) $400 \mu \mathrm{g} / 12 \mu \mathrm{g}$ via the Turbuhaler ${ }^{\circledR}$ in patients with advanced COPD (Lipson et al., Am J Respir Crit Care Med. 2017 Aug 15;196(4):438-446). Given the clinical overlap of exacerbations and pneumonia, we analysed composites of these outcomes to assess the overall benefit/risk of FF/ UMEC/VI compared with BUD/FOR.

Methods: We performed post-hoc analyses in the ITT (24 weeks; FF/ UMEC/VI, n=911; BUD/FOR, $\mathrm{n}=899$ ) and extension (EXT; 52 weeks; FF/ $\mathrm{UMEC} / \mathrm{VI}, \mathrm{n}=210 ; \mathrm{BUD} / \mathrm{FOR}, \mathrm{n}=220$ ) populations. There were two composite outcomes: time-to-first investigator-reported moderate (required antibiotics or oral/systemic corticosteroids)/severe (required hospitalisation) exacerbation or pneumonia event (investigator reported by a prespecified list of preferred terms); and time-to-first severe exacerbation/hospitalised pneumonia event. Analyses were based on a proportional hazards model.

Results: For moderate/severe exacerbation or pneumonia, FF/UMEC/ $\mathrm{VI}$ had a statistically significant reduction in risk vs BUD/FOR of $25 \%$ (ITT; 109 [12\%] vs 130 [14\%]; $p=0.027)$ and 44\% (EXT; 37 [18\%] vs 63 [29\%]; $\mathrm{p}=0.006$ ). For severe exacerbation/hospitalised pneumonia, FF/ UMEC/VI had a lower risk vs BUD/FOR of $17 \%$ (ITT; 22 [2\%] vs 24 [3\%]; $\mathrm{p}=0.541)$ and $65 \%$ (EXT; 8 [4\%] vs $23[10 \%] ; \mathrm{p}=0.010)$.

Conclusion: The results of these exacerbation/pneumonia composite outcomes support a favourable benefit/risk profile of once-daily $\mathrm{FF} / \mathrm{UMEC} / \mathrm{VI}$ compared with BUD/FOR in patients with COPD.

\section{PS087}

Antikoagulation als interdisziplinäre Herausforderung - Unterstützung klinischer Entscheidungen durch medizinische Apps

\author{
Hans-Jörg Hippe'; Mark Lüdde'; Samuel Tobias Sossalla²
}

'Universitätsklinikum Schleswig-Holstein Campus Kiel, Kiel; ${ }^{2}$ Universitätsklinikum Regensburg, Regensburg

Hintergrund: Die Möglichkeiten der Digitalisierung haben bisher kaum Einzug gehalten in den ärztlichen Arbeitsalltag. Es gibt zwar mehr als 100.000 Gesundheits-Apps für Patienten und Ärzte. Allerdings ist unklar, welche davon, Patienten helfen und Ärzte entlasten. Die klinischen Entscheidungsprozesse sind im Bereich Antikoagulation außerordentlich komplex geworden und dabei zunehmend personalisiert. Medizinische Smartphone-Applikationen („Medical-Apps“) können hier möglicherweise einen Vorteil bieten.

Methoden: Am Beispiel zweier Smartphone-Apps zur Antikoagulation soll beschrieben werden, wie Ausbildung, Patientenversorgung und Patientensicherheit bei der schwierigen Therapie mit Antikoagulantien verbessert werden kann. Die App „DAPT-Advisor" unterstützt Ärzte bei der Auswahl der passenden Plättchen- und Gerinnungshemmung nach Stentimplantation und anderen kardialen Eingriffen, inkl. TAVI und MitraClip. Dabei wählt der Nutzer eine bestimmte Patientenkonstellation aus, z.B. den Stenttyp, das Ischämie-Risiko (elektiv/ACS) oder ggfs. die Indikation zur oralen Antikoagulation. Als Ergebnis erhält der Nutzer eine konkrete Patienten-individuelle und Eingriff-spezifische Therapieempfehlung zur Plättchenhemmung und Antikoagulation.

Die zweite App greift das Thema orale Antikoagulation bei Vorhofflimmern und venöser Thromboembolie (TVT/LAE) auf, insbesondere mit den neuen Nicht-Vitamin K-abhängigen oralen Antikoagulantien (NOAK). Hier unterstützt die "NOAC-Advisor" - App die behandelnden Ärzte mit konkreten Vorschlägen zu den Fragen "Welches Präparat? Welche Dosis? Wie lange? Für welchen Patienten?" und zwar Patienten-individuell nach Alter, Nierenfunktion, Blutungsrisiko und spezifischen Begleiterkrankungen. Zusätzlich bietet die App viele praktische Hinweise zur Anwendung der Gerinnungshemmer im Alltag, z.B.: Management von Blutungen, Interaktionen mit anderen Arzneimitteln, perioperatives „Bridging“, Vorgehen bei Klappenerkrankungen oder einer zusätzlichen Plättchenhemmung („Triple-Therapie“).

Beide Apps sind von klinisch tätigen Kardiologen entwickelt worden und basieren auf den aktuellen Leitlinien der Fachgesellschaften. Anders als viele Gesundheits-Apps sind sie als Medizinprodukte CE-zertifiziert. Beide Apps vermitteln damit auf zeitgemäße und transparente Weise praxisrelevantes Wissen, das sich auch zur Ausbildung von Medizinstudenten, PJ-Studenten und Assistenzärzten eignet. Dabei werden keine personenbezogenen Daten wie Name, Geburtsdatum oder Wohnort in der App abgefragt.

Zusammenfassung: Plättchenhemmung und Antikoagulation sind enorme Herausforderungen für den Internisten ebenso wie für Chirurgen, Neurologen und Hausärzte im praktischen Alltag. Medizinische Smartphone-Apps demonstrieren hier beispielhaft wie eHealth die ärztliche Entscheidung und Ausbildung junger Ärzte unterstützen kann und dabei die Patientensicherheit nachhaltig verbessern könnte. 


\section{PS088}

\section{Postpartales protrahiertes Nierenversagen und Delir als Aus- druck einer Hypophyseninsuffizienz}

Melanie Kandulski'; Christina Selgrad ${ }^{2}$; Michael Selgrad'; Tanja Bergmann'; Olaf Ortmann ${ }^{2}$; Martina Müller ${ }^{1}$

${ }^{1}$ Universitätsklinikum Regensburg, Regensburg; ${ }^{2}$ Caritas-Krankenhaus St. Josef, Regensburg

Vorgeschichte: Eine 38-jährige Patientin wurde uns mit dem Verdacht eines hämolytisch urämischen Syndroms (HUS) bei unklarem akuten Nierenversagen und Delir aus einem auswärtigen Krankenhaus zugewiesen. Fünf Wochen zuvor wurde die Patientin in der 34. Schwangerschaftswoche aufgrund eines beginnenden HELLP-Syndroms per Notfallsectio von Zwillingen entbunden. Intraoperativ entwickelte sich eine atone Uterusnachblutung mit hämorrhagischem Schock und Verbrauchskoagulopathie. Aufgrund von Nachblutungen mit Hämatombildung erfolgten mehrere Wundrevisionen. Im Verlauf entwickelten sich ein protrahiertes Nierenversagen mit Dialysepflichtigkeit und ein Delir.

Diagnostik \& Verlauf: Bei Aufnahme zeigte sich die Patientin in einem stuporösen Zustand bei Kreislaufstabilität. Laborchemisch ergab sich kein Hinweis auf das Vorliegen eines HUS. Auffällig waren eine neu aufgetretene thyreotrope Insuffizienz und eine ausgeprägte Polyurie nach Beendigung der kontinuierlichen Dialysetherapie. Der Cortisolspiegel zeigte sich mit 1,1 g/dl deutlich unterhalb des Normbereichs. Unter dem Einsatz von Hydrocortison zeigte sich die Patientin nach wenigen Stunden orientiert. Unter Desmopressin wurde konzentrierter Urin ausgeschieden. Ein MRT des Schädels zeigte eine kleine Defektzone der Hypophyse linksseitig, vereinbar mit einer Einblutung oder Apoplexie, woraufhin die Diagnose eines Sheehan-Syndroms gestellt werden konnte.

Fazit: Postpartal sollte nach hohem Blutverlust, protrahiertem Krankheitsverlauf mit Delir und Nierenversagen immer auch an das seltene Krankheitsbild des Sheehan-Syndroms gedacht werden.

\section{PS089 \\ Akute kardiale Dekompensation bei supraventrikulärer Tachy- kardie und hypertensiver Blutdruckentgleisung: Zwei Fallberich- te eines Phäochromocytoms}

Henry Kanthak; Armin Wöhrle; André Schneider; Matthias Leschke

Klinikum Esslingen, Esslingen

Zwei Patientinnen stellten sich im Alter von 54 (Pat. 1) und 46 Jahren (Pat. 2) mit heftigen, thorakalen Beschwerden, ausgeprägter Hypertonie, Sinustachykardie und akuter Linksherzdekompensation mit erhöhten Troponinwerten unter der Verdachtsdiagnose eines akuten Koronarsyndroms in unserer Klinik vor. Die erste Pat. zeigte echokardiographisch das typische Bild einer Tako-Tsubo-Kardiomyopathie mit weitgehender Akinesie der Vorderwand, hochgradig eingeschränkter linksventrikulärer Pumpfunktion und dem Nachweis von linksventrikulären Thromben. Die zweite Pat. wies eine Sinustachykardie mit Frequenzen um 140/min und systolischen Blutdruckwerten um $220 \mathrm{mmHg}$ bei echokardiographisch hochgradig, diffus eingeschränkter linksventrikulärer Pumpfunktion auf. Unter der Verdachtsdiagnose eines akuten Koronarsyndroms mit erhöhten Troponinwerten erfolgte bei beiden Pat. angiographisch der Ausschluss einer koronaren Herzerkrankung.

Die zweite Pat. entwickelte kurzfristig einen fulminanten kardiogenen Schock mit der Notwendigkeit der Intubation. Bei zunehmender
Katecholaminpflichtigkeit erfolgte die Anlage einer Impella-Coaxial-Pumpe. Nach zwischenzeitiger Kreislaufstabilisierung musste die Patientin im progredienten Schock mit Laktatazidose zur ECMO-Anlage verlegt werden. Beide Pat. zeigten sonographisch bzw. im CT-Abdomen eine Raumforderung im Bereich der Nebenniere, so dass bei Tachykardie und hypertensiver Entgleisung der klinische Verdacht auf ein Phäochromocytom geäußert werden musste. Beide Pat. wiesen massiv erhöhte Metanephrin- und Normetanephrinwerte auf. Bei beiden Pat. erfolgte zunächst eine zentrale alpha-Blockade mittels Doxazosin mit anschließender ß-Blockertherapie, darunter normalisierten sich die Blutdruckwerte. Herzchirurgisch musste bei der ersten Patientin notfallmäßig eine Entfernung der Ventrikelthromben und im Intervall eine Adrenektomie erfolgen. Bei der zweiten Patientin erfolgte eine angiographische Embolisation des Nebennierentumors mit späterer Resektion.

Fazit: Sinustachykardie und krisenhaft erhöhte Blutdruckwerte trotz einer hochgradig eingeschränkten linksventrikulären Funktion sind hochverdächtig für ein Phäochromocytom. Die linksventrikuläre Dysfunktion kann sich unter dem Bild einer Tako-Tsubo-Kardiomyopathie, aber auch primär als hochgradig diffus eingeschränkte linksventrikuläre Funktion mit drohendem kardiogenen Schock präsentieren

\section{PS090 \\ Regulation der microRNA-Expression durch p73-Isoformen beim hepatozellulären Karzinom}

Lisa Kaser; Elisabeth Aschenbrenner; Kirstin Pollinger; Claudia Kunst; Martina Müller

Universitätsklinikum Regensburg, Sulzbach-Rosenberg

Einleitung: p53 gehört zu den am häufigsten mutierten Genen in Tumorzellen. Neben p53 gehören auch p63 und p73 zur p53-Familie von Transkriptionsfaktoren. Diese reagieren auf zelluläre Stresssignale unter anderem durch Induktion von Zellzyklusarrest und Apoptose. p63 und p73 nehmen in der Zelle zum Teil p53-analoge Funktionen wahr, können jedoch auch gegensätzliche Effekte haben. Je nach Splicevariante - mit Transaktivierungsdomäne (TA) oder in dominant negativer Form (DN) - und den Eigenschaften der spezifischen Bindungsstelle führen p53-Proteine zu einer Aktivierung oder Inhibition des betreffenden Zielgens. MicroRNAs sind kleine, nicht-codierende RNA-Moleküle mit einer Länge von ca. 22 Nukleotiden, die eine wichtige Rolle bei der Genregulation spielen. Die Expressionsprofile von microRNAs können wiederum durch die p53-Familie beeinflusst werden. Über die p73-abhängige Expression von microRNAs beim hepatozellulären Karzinom (HCC) ist bisher wenig bekannt. Ziel der Arbeit war deshalb die Identifizierung von microRNAs, die beim HCC durch p73 und dessen Isoformen reguliert werden.

Methoden: Hep3B-Zellen wurden mit rAd-TAp73, rAd- $\Delta N p 73$ oder rAd-GFP transfiziert. Die TAp73- und $\triangle N p 73$-abhängige microRNA-Expression wurde mittels qPCR bestimmt. Zur Analyse von Effekten HCC-relevanter Therapeutika auf die TA-/ $\Delta$ Np73-abhängige microRNA-Regulation wurden transfizierte Zellen in vitro bis $\mathrm{zu} 72 \mathrm{~h}$ mit Doxorubicin, Bleomycin, Regorafenib, Sorafenib und Tivantinib inkubiert.

Ergebnisse: $48 \mathrm{~h}$ nach Transfektion führte die Überexpression von TAp73 zu einer starken Induktion von miR-34a (6,5-fach), miR-145 (6,9fach) und miR-149 (8,1-fach). Durch Inkubation mit Tivantinib wurde die TAp73-abhängige Expression von miR34a noch weiter gesteigert. Die Überexpression von $\Delta N p 73$ führte nach $48 \mathrm{~h}$ hingegen nur zu einer leichten Steigerung der Expression von miR-34a, miR-145 und miR-149 (maximal 3,2-fach). Nach Inkubation mit HCC-relevanten Wirksubs- 
tanzen waren die Spiegel dieser microRNAs unverändert oder sogar reduziert im Vergleich zu unbehandelten Zellen.

Schlussfolgerung: p73-Isoformen haben divergente Effekte auf die microRNA-Profile im HCC. TAp73 ist ein bedeutender Regulator der microRNAs 34a, 145 und 149. Darüber hinaus deuten unsere Daten auf eine synergistische Wirkung der TAp73-Überexpression und Chemotherapeutika bzw. „targeted therapies" hin. Die Regulation tumorsuppressiver microRNAs scheint daher ein TAp73-vermittelter Effektormechanismus bei Tumorentstehung und Therapieansprechen zu sein und könnte neue Optionen zur Entwicklung therapeutischer und prognostischer Strategien beim HCC darstellen.

\section{Innere Medizin integral II}

\section{PS091 \\ Ein Prognosescore für das Überleben nach Lungentransplanta- tion}

Andreas Kümmel'; Tobias Reichert'; Lars Henning Schmidt ${ }^{2}$; Roland Buhl'

'Universitätsmedizin Mainz, Mainz; ${ }^{2}$ Universitätsklinikum Münster, Münster

Die Lungentransplantation ist eine wichtige Therapieoption in der Behandlung terminaler Lungenerkrankungen. Aufgrund der geringen Zahl der Spenderorgane ist es wichtig, die potentiellen Empfänger zu identifizieren, die nach einer Transplantation möglichst lange überleben. Wir untersuchten retrospektiv die Daten von 103 Patienten, die in der Universitätsmedizin Mainz zwischen 1995 und 2016 zum ersten Mal transplantiert wurden. Das Patientenkollektiv teilten wir in eine Trainingskohorte $(n=51)$ und eine Kontrollkohorte $(n=52)$ auf. In der Trainingskohorte entwickelten wir einen Summenscore aus den Elementen: Aortenklappenvitium, Mitralklappenvitium, Osteoporose, diastolische Dysfunktion und Analgetika-Dauertherapie („AMODDA“, min o, max 5 Punkte). Patienten mit o oder 1 Punkt im AMODDA-Score hatten ein medianes Überleben von 199 Monaten, Patienten mit 2 oder mehr Punkten dagegen nur 42 Monate $(p=0,007)$. Dies konnten wir in der Kontrollkohorte bestätigen $(p=0,027)$.Mit dem AMODDA-Score können Kandidaten mit guten Langzeiterfolgsaussichten für eine Lungentransplantation ausgewählt werden.

\section{PS092 RDS- Symptome bei Patienten mit funktioneller Dyspep- sie - positiv beeinflusst durch Menthacarin}

Ahmed Madisch'; Berenike Stracke ${ }^{2}$

${ }^{1}$ Klinikum Region Hannover - Klinikum Siloah, Hannover; ${ }^{2}$ Dr. Willmar Schwabe GmbH \& Co. KG, Karlsruhe

Hintergrund: Funktionelle gastrointestinale Beschwerden (FGIB) zeichnen sich durch das Fehlen organischer Ursachen sowie interindividuelle Unterschiede bei Auftreten und Ausprägungsgrad der Symptome aus. Die funktionelle Dyspepsie (FD) sowie das Reizdarmsyndrom (RDS) gehören zu den häufigsten FGIBs. Bis heute ist die Pathophysiologie von FGIDs nicht vollständig geklärt. Es ist bekannt, dass symptomatische und pathophysiologische Überlappungen zwischen RDS und FD existieren. FD und RDS verursachen oft einen erheblichen Leidensdruck.

Ziel: Die Wirksamkeit und Verträglichkeit von Menthacarin* - eine proprietäre Kombination ätherischer Öle mit spezifizierter Qualität aus Mentha x piperita L. (90 mg WS ${ }^{\circledast} 1340$ ) und Carum carvi L. $(50 \mathrm{mg}$
WS ${ }^{\circledR} 1520$ ) - wurden bei Patienten mit funktioneller Dyspepsie (FD) bereits gezeigt. In einer Subgruppenanalyse wird der Einfluss von Menthacarin auf die FD-assoziierten RDS-Symptome bei diesen Patienten untersucht.

Methode: Auf Basis aller mit Menthacarin bisher durchgeführten Doppelblindstudien wurde eine deskriptive Subgruppenanalyse durchgeführt. In die Analyse eingeschlossen wurden Patienten, bei denen typische Reizdarm-Symptome wie Druck-, Schwere- und Völlegefühl, Flatulenz und Diarrhoe auftraten.

Ergebnisse: Fünf randomisierte Doppelblindstudien mit einer Behandlungsdauer von jeweils 28 Tagen wurden analysiert. Drei Studien (Studie A, B und C) mit insgesamt 376 Patienten waren für die Analyse geeignet. Davon litten $30 \%$ der Patienten (111) an einem begleitenden RDS. Unter der Einnahme von Menthacarin verbesserten sich die RDS assoziierten Symptome Druck-, Schwere- und Völlegefühl um 1,9 $\pm 1,1$ Punkte (Mittelwert \pm Standardabweichung, Studie A), 1,3 $\pm 0,8$ (B), 0,6 $\pm 0,9$ (C) Flatulenz um 1,4 $\pm 0,7(A), 1,4 \pm 1,0$ (B), $0,8 \pm 1,1$ (C) und Diarrhoe um $0,4 \pm 0,7(A), 0,7 \pm 0,7$ (B), 0,2 $\pm 0,8$ (C) Punkte - ermittelt über eine Selbst-Beurteilungsskala des Schweregrades (o: nicht vorhanden bis 3: schwer). Die Verbesserung der RDS assoziierten Symptome Druck-, Schwere- und Völlegefühl sowie Flatulenz war unter Menthacarin etwa doppelt so stark ausgeprägt wie unter Placebo.

Schlussfolgerung: Menthacarin reduziert begleitende RDS-Symptome bei Patienten mit FD und könnte eine Behandlungsoption bei RDS-Patienten darstellen.

${ }^{*}$ Menthacarin ${ }^{\circledast}$ ist der Wirkstoff des Produkts Carmenthin ${ }^{\circledast}$ (Dr. Willmar Schwabe GmbH \& Co. KG, Karlsruhe)

\section{PS093}

Prädiktive Bedeutung des endosonographischen (EUS) Stagings bei lokal fortgeschrittenem Adenokarzinom des Magens (MC) oder ösophagogastralen Übergangs (AEG) - Analyse von Daten einer perioperativen AIO-CAO Phase II Studie

Visvakanth Sivanathan'; Michael Stahl ${ }^{2}$; Christoph Utz; Annett Maderer'; Florian Lordick ${ }^{3}$; André Mihaljevic ${ }^{4}$; Stephan Kanzler ${ }^{5}$; Thomas Höhler ${ }^{6}$; Peter Thuss-Patience ; Stefan Mönig ${ }^{8}$; Volker Kunzmann ${ }^{9}$; Sebastian Schroll ${ }^{10}$; Andreas Sandermann ${ }^{11} ;$ Andrea Tannapfel ${ }^{12}$; Hans-Joachim Meyer ${ }^{13} ;$ Chris- $^{-}$ toph Schuhmacher ${ }^{4}$; Hans Jochen Wilke ${ }^{14}$; Katrin Krause ${ }^{15}$; Markus Möhler ${ }^{1}$

${ }^{1}$ Universitätsmedizin Mainz, Mainz; ${ }^{2}$ Universitätsklinikum Essen, Essen; ${ }^{3}$ Universitätsklinikum Leipzig, Leipzig; ${ }^{4}$ Klinikum rechts der Isar der TU München, München; ${ }^{5}$ Leopoldina Krankenhaus Schweinfurt, Schweinfurt; ${ }^{6}$ Prosper Hospital Recklinghausen, Recklinghausen; ${ }^{7}$ Charité Universitätsmedizin Berlin, Berlin; ${ }^{8}$ Universitätsklinikum Köln, Köln; ${ }^{9}$ Universitätsklinikum Würzburg, Würzburg; ${ }^{10}$ Klinikum Braunschweig, Braunschweig; ${ }^{11}$ Wissenschaftlicher Service Pharma GmbH, Langenfeld; ${ }^{12}$ Ruhr-Universität Bochum, Bochum; ${ }^{13}$ Deutsche Gesellschaft für Chirurgie, Berlin; ${ }^{14}$ Kliniken Essen-Mitte, Essen; ${ }^{15}$ Arbeitsgemeinschaft Internistische Onkologie in der Deutschen Krebsgesellschaft e.V.

Einleitung/Ziele: In dieser Studie wurde die zusätzliche Gabe von Panitumumab zur ECX-Basistherapie (Epirubicin, Cisplatin and Capecitabine) bei MC oder AEG untersucht. Diese Auswertung korrelierte die präoperative EUS nach neoadjuvanter CTx zusätzlich zur initialen Untersuchung (Basis-EUS) mit dem Therapieansprechen.

Methoden: 160 Patienten(Pat.) aus 22 Zentren wurden eingeschlossen. Die präoperativen uT-/uN-Stadien wurden mit dem histopathologischen $\mathrm{pT} / \mathrm{pN}$-Stadien verglichen. Jede Reduktion im T-Stadium der präoperativen EUS zur Basis-EUS wurde als Downstaging (DS+) bewertet und mit der histopathologischen Regression (Grad 1-2 nach 
Becker) sowie dem Gesamtüberleben (ÜL) der Pat. ohne Downstaging (DS-) verglichen. Der präoperative N-Status (positiv N+ oder negativ $\mathrm{N}-$ ) wurde mit dem ÜL korreliert.

Ergebnisse: In $48 \%$ korrelierte das präoperative EUS-T- mit dem pT-Stadium (Sensitivität (Sens.) 48\%, Spezifität (Spez.) 52\%) und bei $64 \%$ das präoperative EUS-N- mit dem pN-Stadium (Sens. $76 \%$, Spez. $52 \%)$. Bei DS+ war eine Tendenz zum verbesserten ÜL nachweisbar (medianes ÜL DS+ 38,5 Monate (M), DS- medianes ÜL in der Beobachtungszeit nicht erreicht (NA), $p=0,18)$. Das ÜL der $\mathrm{N}+$ Pat. (medianes ÜL 36,1 M, p= 0.0045) war im Vergleich (i.V.) zu den N- schlechter (NA).

Schlussfolgerung: Trotz multizentrischer Beteiligung und unterschiedlicher EUS-Geräte zeigte diese große prospektive Arbeit eine zu anderen Studien vergleichbare diagnostische Genauigkeit der Endosonographie. Der präoperative positive EUS-N-Status ist prädiktiv für eine schlechte Prognose.

\section{PS094}

\section{Doss-Porphyrie (ALAD-Porphyrie) durch Promotordefekt}

Muhidien Soufi'; Volker Ruppert ${ }^{1}$; Tobias Müller'; Tanja Nickolaus'; Volker Ziller'; Margareta Frank'; ; Jürgen Schäfer ${ }^{1}$

\section{'Universitätsklinikum Gießen-Marburg, Standort Marburg, Marburg; ${ }^{2}$ Medizinisches Versorgung Zentrum (MVZ) Innere Medizin, Marburg, Marburg}

Hintergrund: Das Krankheitsbild der Porphyrien stellt eine Gruppe von seltenen Stoffwechselerkrankungen der Hämbiosynthese dar. Durch verminderte Enzymaktivitäten kommt es zur Akkumulation von Porphyrinvorläufern. Betroffene Patienten entwickeln im akuten Stadium ein buntes klinisches Syndrom mit abdomineller, neurologischer und kardiovaskulärer Symptomatik. Eine sehr seltene Form der Porphyrie ist die autosomal rezessiv vererbte Doss-Porphyrie. Hier kommt es durch Defekte der ALA-Dehydratase (ALAD) zu einer Akkumulation von $\delta$-Aminolävulinsäure. Wir berichten über eine 31 Jährige Patientin mit wiederkehrenden gastrointestinalen Beschwerden die über nahezu 10 Jahre hinweg trotz umfassender gastroenterologischer Diagnostik ohne fassbare Ursache blieb. Während zweier Schwangerschaften war die Patientin völlig beschwerdefrei. Auch steroidhaltige Asthmasprays führten zur Linderung der Beschwerden. Eine von uns durchgeführte Porphyrie-Diagnostik ergab den Hinweis auf eine (milde) Doss-Porphyrie. Dabei konnten wir einen neuartigen Defekt im ALAD- Promotor nachweisen.

Methoden: Messung von Phorphobilinogen, $\delta$-Aminolävulinsäure. DNA-Sequenzierung der Gene von ALA-Synthase (ALAS), PBG-Desaminase (PBGD) und ALA-Dehydratase (ALAD). Klonierung von Promotorsequenzen in Luziferase-Reportergene. Ko-Transfektion mit Östrogen/Glukokortikoidrezeptor-Expressionsvektoren. Messung der Promotoraktivität in HEK-293-Zellen.

Ergebnisse: Die Porphyriediagnostik ermittelte bei bestehenden Beschwerden eine mehr als 4 fache Erhöhung der $\delta$-Aminolävulinsäure bei gleichzeitig erniedrigten PBG. Es fanden sich keine kodierenden Mutationen in den untersuchten Genen. Im schmerzfreien Intervall war die ALAD Konzentration zwar erniedrigt, deren Aktivität jedoch normal. Im ALAD-Promotor identifizierten wir 3 unbekannte SNPs. Die Messung der Promotoraktivität in Gegenwart von Glukokortikoid/ Östrogenrezeptor ermittelte basal eine um 2,5fach erniedrigte Promotoraktivität im Vergleich zum Wildtyp-Promotor $(p=0,001)$. Nach Stimulation mit Hormon erhöhte sich die ALAD-Promotoraktivität um das 1.5fache vom Basalwert des Wildtyp-Promotors $(p=0,001)$.
Diskussion: Der Fall unserer Patientin zeigt erstmalig einen Einfluss von Promotor-SNPs auf die Aktivität der ALAD bei der Entstehung einer Doss-Porphyrie. Gemäß der diagnostischen Zielführung für akute Porphyrien führten wir nach Bestimmung von Phorphobilinogen, $\delta$-Aminolävulinsäure ein genetisches Screening der Gene von ALAS, PBG und ALAD durch. Unsere Untersuchungen identifizierten 3 unbekannte SNPs im ALAD-Promotor, welche in unmittelbarer Nachbarschaft zu Steroidrezeptor-Bindungsstellen liegen. Interessanterweise war unsere Patientin während Schwangerschaftszeiten, Kontrazeption und bei Anwendung steroidhaltiger Sprays weitestgehend beschwerdefrei. Aufgrund dieser Hinweise führten wir Stimulationstests durch, die eine sehr gute Ansprechrate auf Steroide zeigten. In unserem Fall handelt es sich um eine milde Form der Doss-Porphyrie.

\section{PS095}

Clinical profile of Hyponatremia in Tertiary Care Center in Kochi Kerala: Retrospective Hospital Based Observational Study

V.S Srikanth; Akash Thomas; Rengitha Nair; Senthilve; vasudevan

\section{Amrita Institute of Medical Sciences, kochi, India}

Background: Hyponatremia is the common of electrolyte abnormality seen in clinical practice. Hyponatremia is associated with altered sensorium, seizures, falls and cognitive dysfunction. Even mild hyponatremia could lead to severe complications and prolonged hospital stays. Understanding the emerging trends in manifestations and treatment of hyponatremia will help in efficient and cost effective management of hyponatremia and allied co morbidities. This study was conducted on in-patients admitted in AIMS hospital with Hyponatremia and to understand the etiology and manifestations of Hyponatremia.

Methods: We have conducted the present study on 980 patients who have got admitted in AIMS, Kochi, Kerala. They were categorized based on serum sodium level under 3 groups of Hyponatremia (Mild, Moderate and Severe). The data collected were analyzed for Clinical presentations, Severity of Hyponatremia in relation to sensorium, Etiology of Hyponatremia, Causes of SIADH, Drugs causing SIADH and Co-morbidities of Hyponatremia.

Results/Observations: Hyponatremia was observed predominantly in the age group $>70 y$ rs (37.3\%), with male predisposition (63\%). Altered sensorium is the most common presentation of hyponatremia. The number of patients with disorientation is more in the moderate hyponatremia $(64 \%)$ compared to severe hyponatremia (20\%). SIADH was observed as leading cause of Hyponatremia. Respiratory causes such as pneumonia, asthma, OAD were the predominant causes of SIADH, followed by Dilutional hyponatremia and Drug Induced Hyponatremia. Of the various types of carcinoma, Lung Cancer and genitourinary cancer were the main causes of SIADH. The major infections associated with hyponatremia were UTI (68\%), Chest Infection (15\%) and Cellulitis (14\%). $42 \%$ population with hyponatremia had Diabetes Mellitus, of which $64 \%$ had peripheral neuropathy and $10 \%$ had complications like diabetic foot and necrotizing fasciitis. Analysis of data related to hypertension showed $51 \%$ population with hyponatremia had hypertension.

Conclusion: This study summarizes the varied presentations of hyponatremia, its causes and co-morbidities which will provide better understanding of hyponatremia. Also aid the physician in diagnosing the precise cause of hyponatremia and better patient management ensuring high quality patient care. 


\section{PS096}

Die Salford Lung Studie Asthma - Überblick über eine randomisiert-kontrollierte Studie zum Vergleich der Wirksamkeit von Fluticasonfuroat/Vilanterol (FF/VI) mit der Standardtherapie im klinischen Alltag

Anna-Maria Stegherr; Bernadette Zölch; Cordula Mohrlang

Glaxo Smith Kline, München; Glaxo Smith Kline, München; Glaxo Smith Kline, München

Hintergrund: Evidenz zur Asthmatherapie beruht auf klassischen randomisiert kontrollierten Studien, die hochselektive Patientengruppen unter streng reglementierten Bedingungen untersuchen. Wegen strikter Ein- und Ausschlusskriterien berücksichtigen diese Studien nur etwa 3\% der Asthmapatienten2. Ergänzend wurde SLS Asthma durchgeführt um die Wirksamkeit verschiedener inhalativer Therapieoptionen im klinischen Alltag zu untersuchen. Die Studie wurde bereits veröffentlicht. (Woodcock A et al. Lancet 2017; 39o(10109):p2247-2255)

Methodik: SLS Asthma ist eine randomisiert kontrollierte Studie, die über 52 Wochen die Wirksamkeit von FF/VI unter Alltagsbedingungen bei 4233 symptomatischen Patienten mit hausärztlicher Asthmadiagnose und inhalativer Dauertherapie mit Kortikosteroiden (ICS) (allein oder kombiniert mit langwirksamen Beta-Agonisten (LABA)) untersucht. Nach Randomisierung wurde die Therapie mit FF/VI einmal täglich (100/25 $\mu \mathrm{g}$ bzw. $200 / 25 \mu \mathrm{g}$ ) begonnen oder die optimierte Standardtherapie (festgelegt vom behandelnden Arzt) fortgeführt. Asthmakontrolle war primärer Endpunkt, bestimmt als Anteil der Responder (Patienten mit einem Anstieg > 3 (MCID) und/oder einem Wert $>20$ im Asthmakontrolltest (ACT) nach 24 Wochen) bei Patienten der ITT mit einem Ausgangswert von $<20$ im ACT $(n=3026)$. Sekundäre Endpunkte beinhalteten u.a. Exazerbationen, Bedarfsmedikationsgebrauch und Lebensqualität (AQLQ). Darüber hinaus wurden Sicherheitsaspekte ausgewertet.

Ergebnisse: Nach Therapieeinleitung mit FF/VI betrug der Responder-Anteil $71 \%$ gegenüber $56 \%$ unter Standardtherapie (Odds Ratio $2,0(95 \% \mathrm{KI} 1,71,2,34 ; \mathrm{p}$

\section{PS097 \\ May Osteopontin (OPN) serve Diagnosis of Hypertrophic Cardi- omyopathy - a pilot project}

Sylvia Swierzewska'; Martin Farr²; Matthias Lutz ${ }^{3}$; Tanja Kottmann ${ }^{4}$; Lothar Faber $^{2}$; Dieter Horstkotte ${ }^{2}$; Norbert Frey ${ }^{3}$; Christoph Langer ${ }^{5}$

${ }^{1}$ St. Barbara-Klinik Hamm-Heessen, Hamm; ${ }^{2} \mathrm{HDZ}-\mathrm{NRW}$, Bad Oeynhausen; ${ }^{3} \mathrm{UKSH}$, Campus Kiel, Kiel; ${ }^{4} \mathrm{Clinical}$ Research Organisation, Hamm; ${ }^{5} \mathrm{Herz}-$ zentrum Bremen, Bremen

Introduction: Hypertrophic cardiomyopathy (HCM) is characterized by myocardial fibrosis. Osteopontin (OPN) has never been investigated in HCM. Osteopontin is a glycoprotein secreated in different tissues and also by cardiac myocytes and fibroblasts. Thus, reflecting fibrogensis within the myocardium OPN serum levels were shown to correlate positive with left-ventricular (LV) muscle mass (MM) and ejection fraction (EF) in patients with heart failure of different etiology. In this project we analyzed OPN in patients with $\mathrm{HCM}$ with regard to different clinical parameters.

Methods: Therefore, we included 137 consecutive HCM-patients presenting different states of disease. Blood samples were drawn from these patients between 2002 and 2007. The corresponding plasma probes were preserved lege artis continuously at $-80^{\circ} \mathrm{C}$. Applying
Enzyme-linked Immunosorbent Assay (ELISA) OPN-levels were assessed under the use of two kits of the same charge. In order to estimate the prognostic potential of OPN, a comparison of the resulting OPN-values for each parameter was drawn individually. Within this project evaluated parameters included the presence of HCM-risk factors (septal thickness, abnormal blood pressure during ergonometry, family history of sudden cardiac death, recurrent syncope, ventricular tachycardia) as well as LV-MM and LV-EF.

Results: Mean age of the study group was $50.07 \pm 15.48$ years (median 51 years), mean body length was $173 \pm 0.1 \mathrm{~cm}$ and mean body mass index $27.35 \pm 4.23$. The correlation of OPN with the presence of HCM risk factors was of marginal significance: $p=0,061$. Osteopontin levels correlated weakly with LVMM $(p=0,850)$ and with LV-gradient at rest and under provocation ( $p=0,62$ and 0,55 , respectively) but significantly with LV-EF: $p=0,011$. In the summarized group of patients presenting with NYHA I + II and NYHA III + IV OPN correlated by $p=0,130$.

Conclusion: In this pilot study HCM did not correlate significantly with the presence of classic HCM-risk factors and LVMM but with reduced LV EF. Further research is recommended.

\section{PS098}

Single inhaler, once-daily, triple therapy (ICS/LAMA/LABA) in patients with advanced COPD: health-related quality of life (HR$\mathrm{QoL}$ ) and activity outcome results from the FULFIL trial

Maggie Tabberer'; David Lomas²; Helen Barnacle'; Ruby Birk'; Anna-Maria Stegherr'; Noushin Brealey'; Chang-Qing Zhu'; Steve Pascoe'; Nicholas Locantore; David Lipson ${ }^{3}$

${ }^{1}$ Glaxo Smith Kline, Uxbridge, United Kingdom; ${ }^{2}$ Univerversity College London, London, United Kingdom; ${ }^{3}$ Glaxo Smith Kline, University of Pennsylvania, King of Prussia, Philadelphia, United States

Rationale: The randomized, phase III FULFIL trial demonstrated statistically significant improvements in lung function, St George's Respiratory Questionnaire (SGRQ) total score, and COPD exacerbations with once-daily fluticasone furoate/umeclidinium/vilanterol (FF/UMEC/VI) $100 \mu \mathrm{g} / 62.5 \mu \mathrm{g} / 25 \mu \mathrm{g}$ via a single ELLIPTA ${ }^{\oplus}$ inhaler versus twice-daily budesonide/formoterol (BUD/FOR) $400 \mu \mathrm{g} / 12 \mu \mathrm{g}$ via the Turbuhaler ${ }^{\circledast}$ in patients aged $\geq 40$ years with advanced, symptomatic COPD at risk of exacerbations (Lipson et al., Am J Respir Crit Care Med. 2017 Aug 15;196(4):438-446). We report results for additional measures of health status: COPD Assessment Test ${ }^{\mathrm{TM}}$ (CAT) score, SGRQ domain scores and measures of physical activity.

Methods: Eligible participants had a screening CAT score $\geq 10$. Participants completed the CAT and SGRQ for COPD at day 1 (D1), week 4 (W4), and week 24 (W24); answered a daily question on activity limitation using an e-diary; and completed Global Ratings of Activity Limitation at $D_{1}$ and $W_{24}$ and the Global Impression of Change in Activity Limitation (compared with previous visit) at all visits.

Results: 1810 participants were included in the intent-to-treat population (FF/UMEC/VI, n=911; BUD/FOR, $n=899$ ). A clinically meaningful improvement in mean CAT score (decrease of $\geq 2$ units from baseline) was observed with FF/UMEC/VI at W4 (-2.0) and W24 (-2.5); the treatment differences compared with BUD/FOR were statistically significant at $\mathrm{W}_{4}(-0.7 ; 95 \%$ confidence interval $[\mathrm{Cl}]-1.2,-0.2 ; \mathrm{p}=0.003)$ and W24 (-0.9; 95\% Cl -1.4, -0.4; p 


\section{PS099}

Bakterielle Mechanismen zur Überwindung der Barriere des Darmepithels bei Leberzirrhose - ein in vitro-Modell

Lorenz Wächter; Marika Haderer; Elisabeth Aschenbrenner; Kirstin Pollinger; Sophie Schlosser; Claudia Kunst; Martina Müller-Schilling

Universitätsklinikum Regensburg, Regensburg

Einleitung: Spontan bakterielle Peritonitis (SBP) ist definiert als Infektion des Bauchwassers (Aszites) bei Leberzirrhose. Anders als bei anderen Infektionen kann bei SBP kein chirurgisch entfernbarer Entzündungsherd identifiziert werden. Aus diesem Grund ist die SBP mit ihrer 1-Jahres Mortalität von bis zu 93\% eine der schwerwiegendsten Komplikationen der Leberzirrhose. Es ist bekannt, dass eine bakterielle Überwucherung des Dünndarms, ein geschädigtes Immunsystem und eine gestörte Darmbarriere bei fortschreitender Leberzirrhose die Translokation von Darmbakterien begünstigen und somit maßgeblich zur SBP beitragen. Im Aszites können vor allem Escherichia coli (E. coli), Enterokokken, Streptokokken und Klebsiellen (K. pneumoniae) als Erreger identifiziert werden. Wie es den Darmbakterien gelingt, die Darmbarriere zu überwinden und inwieweit bestimmten Bakterien diesbezüglich eine Sonderstellung zukommt, ist bisher noch unklar. Ziel der Arbeit war die Aufklärung der der bakteriellen Translokation zugrundeliegenden Mechanismen bei SBP mit Hilfe eines in vitro-Modells.

Methoden: Zellen der humanen Darmepithelzelllinien Caco-2 (p53-defizient) und HCT-116 (p53 wt) wurden nach einem Zellwachstum von 6 bzw. 15 Tagen in vitro mit E.coli und K. pneumoniae in verschiedenen $\mathrm{MOI}$ (MOI 0, 1, 5 und 10) für 4 h koinkubiert. Die Effekte auf Zell-Zell-Kontakt vermittelnde Proteine (E-Cadherin, Occludin, Claudine) sowie auf die p53-Familie von Transkriptionsfaktoren wurden mittels Western Blot, qPCR und Fluoreszenzmikroskopie analysiert. Eine Koinkubation mit hitzeinaktivierten Bakterien diente jeweils als Kontrollansatz.

Ergebnis: Die Koinkubation mit E. coli und K. pneumoniae führte an Tag 6 bei einer Konzentration von MOI 5 zu einer Reduktion von Occludin, E-Cadherin und Claudin-1. Neben den Zell-Zell-Kontakten wurde auch p73 bei einer bakteriellen MOI von 5 reduziert. Die jeweiligen Reduktionen zeigten sich im Western Blot und mittels Fluoreszenzmikroskopie, nicht jedoch auf mRNA Ebene. An Tag 15 erzielte bereits eine geringere bakterielle Stimulation (MOI 1) eine Reduktion der Zell-Zell-Kontakte. Vergleichend zu Caco-2 Zellen führten geringe Bakterienkonzentrationen $(\mathrm{MOI}=1)$ an Tag 6 bei HCT-116 Zellen zu destabilisierenden Effekte auf die Integrität des Darmepithels. Bakterielle Konzentrationen von MOI 5 wirkten auf die HCT-116 Zellen letal. Hitzeinaktivierte Bakterien hatten keinen Einfluss auf die Zell-Zell-Kontakte oder p73.

Schlussfolgerung: Bakterien haben destabilisierende Effekte auf die Integrität der epithelialen Barriere und besitzen darüber hinaus einen regulatorischen Einfluss auf die p53-Familie von Transkriptionsfaktoren. Diese beiden Prozesse könnten Teile eines Mechanismus darstellen, der eindringende Bakterien vor einer Immunantwort schützt und damit deren Translokation erleichtert.

\section{Interdisziplinarität in der Inneren Medizin I}

\section{PS100}

Attended vs. Unattended blood pressure measurement in a real environment

Frederic Bauer'; Felix S. Seibert'; Benjamin Rohn'; Klaus Anton Rüdiger Bauer $^{2}$; Eckart Rolshoven ${ }^{2}$; Nina Babel'; Timm Henning Westhoff ${ }^{1}$

\section{'St. Elisabethgruppe, Herne}

The debate on the generalizability of the SPRINT trial's findings raised considerable interest in the technique of unattended office blood pressure (BP) measurement. It remains elusive, however, whether unattended BP measurement yields lower values than conventional measurements in a "real world setting" with subjects consulting their personal general practitioner in a familiar office. We performed a cross-sectional study in 158 patients in four general practitioners' offices and compared conventional auscultatory office BP to unattended automated office BP in 107 subjects (group 1 ) and unattended to attended automated office BP in another 51 subjects (group 2). Unattended BP was calculated as the mean of three automated measurements performed in a separate room after 5 min of rest. Additionally, patients documented home BP for seven days after the consultation. Mean auscultatory office, unattended office and home BP were $144.6 / 81.0,144.1 / 79.9$, and $135.5 / 78.3 \mathrm{mmHg}$ in group 1; unattended and attended automated office blood pressure were 134.2/80.6 and $135.7 / 80.6 \mathrm{mmHg}$ in group 2. Systolic attended and unattended office $B P$ values were significantly higher than home $B P(p<0.001, p<0.01$, respectively). Attended and unattended office $B P$, however, did neither show a significant difference in group 1 nor in group $2(p>0.05$ each). Bland Altman analysis revealed a bias of $0.5 \mathrm{mmHg}$ systolic and $1.1 \mathrm{mmHg}$ diastolic in group 1 and $-1.5 \mathrm{mmHg}$ systolic and o $\mathrm{mmHg}$ diastolic in group 2 . In conclusion, the present findings show that unattended and attended office BP measurements achieve comparable results, if measurements take place at a familiar general practitioner's office.

\section{PS101 \\ Viszeralmedizinische Schmerztherapie - SOP-Entwurf für ein adäquates modernes Vorgehen}

Michael Brinkers; Giselher Pfau; Frank Meyer

Universitätsklinikum Magdeburg A.ö.R., Magdeburg

Hintergrund: Seit 2001 hat sich gezeigt, dass in den somatischen Fächern mit Konsilen klassischer Prägung keine hinreichenden Erfolge in der Schmerztherapie zu erzielen sind. Ziel war es daher, re-organisierend mehr im Liaisonmodus zu arbeiten und zunächst qualitative Aspekte zu eruieren als auch eine mögliche Quantifizierung probatorisch abzuklären.

Material und Methoden: Zunächst wurde für die Ärzte der Anästhesie die Schmerztherapie schematisiert i.S. einer "SOP" (Dokument des klinikinternen Qualitätsmanagements) und über ein Manual jederzeit verfügbar gemacht. Auf Seiten der Allgemein- und Viszeralchirurgen wurde zugelassen, dass die Ärzte der Anästhesie selbständig die Vorschläge auch anordnen durften, parallel zur Arbeit der chirurgischen Stationsärzte.

Ergebnisse (Eckpunkte): Durch dieses „parallele“ Vorgehen wurde erreicht: 
1. Die Konsiliarii sahen die Patienten über einen längeren Zeitraum als sonst bei Konsilen üblich, um den Therapieeffekt/-erfolg abzusichern.

2. Für die Patienten wurden keine realitätsfernen Ratschläge im Konsil erteilt.

3. Die Ratschläge wurden 1:1 umgesetzt.

4. Die Konsiliarii übernehmen die Verantwortung für die Folgen ihrer Ratschläge.

5. Die Arbeitsgruppe der SOP aus Chirurgen und Ärzten der Schmerzambulanz kann nun dazu übergehen, die Erfahrungen aus den letzten Jahren auf die Ärzte der Chirurgie zu übertragen.

6. Mittels systematischer Erfassung durch ein perioperatives/-interventionelles Parameterspektrum sollen im Weiteren die qualitativ favorisierbaren Positiverfahrungen mit der angelaufenen Umsetzung der "SOP Schmerztherapie" nunmehr quantifiziert werden.

Schlussfolgerungen: Die erarbeiteten Algorithmen dienen in einem 1. Schritt dazu, Schmerztherapie reliabel und dennoch individuell handhabbar zu machen. In einem 2. Schritt sollen die Chirurgen noch besser und individueller befähigt werden, selbst bis zu einem gewissen Grade schmerztherapeutisch tätig zu werden (außerhalb des „Metamizol- / Piritramid-Schemas[-Rahmens]"). Dies ist die Grundlage für den 3. Schritt, dass das allgemeine Schmerzniveau auf den chirurgischen Stationen auf ein Mindestmaß gesenkt werden kann als konkret gesetztes Ziel nicht zuletzt im Rahmen des Qualitätsmanagements der Klinik. In einem 4. Schritt sollten die Konsile perspektivisch seltener werden ohne Nachteil für die Effektivität der Schmerztherapie, d.h. bei gleichzeitig optimaler schmerztherapeutischer Betreuung der Stationen - unabhängig vom Personal.

\section{PS102}

24-week efficacy and safety of sotagliflozin, a dual SGLT1 and SGLT2 inhibitor, as adjunct therapy to insulin in type 1 diabetes (inTandem2; NCT02421510)

Thomas Danne'; Bertrand Cariou ${ }^{2}$; Sangeeta Sawhney ${ }^{3}$; Strumph Paul ${ }^{3}$

'Diabetes Center for Children and Adolescents, AUF DER BULT, Kinder- und Jugendkrankenhaus, Hannover, Hannover; ${ }^{2}$ Department of Endocrinology, I'Institut du Thorax CHU Nantes, Nantes, France, Nantes, France; ${ }^{3}$ Lexicon Pharmaceuticals, Inc., The Woodlands, TX, The Woodlands, United States

Question: Sotagliflozin (SOTA) is a dual SGLT1 and SGLT2 inhibitor in Phase 3 development for type 2 diabetes (T2D) and as adjunct therapy to insulin in type 1 diabetes (T1D). SGLT1 inhibition delays and reduces glucose absorption in the proximal intestine, improving postprandial glycemic control. SGLT2 inhibition reduces renal glucose reabsorption.

Methodology: In a double-blind, 52-week international Phase 3 trial, 782 adults with $T_{1} D$ treated with MDI or pump therapy, with $A_{1} C$ 7.0-11.0\% at Screening, were randomized 1:1:1 to placebo, SOTA 200 or $400 \mathrm{mg}$ after a 6-week insulin optimization period. Baseline characteristics were comparable among groups. The primary outcome was change from Baseline in $\mathrm{A}_{1} \mathrm{C}$ at 24 weeks.

Results: SOTA 200 and $400 \mathrm{mg}$ were statistically significant vs. placebo in lowering $A_{1} C$ and achieving the prespecified net benefit endpoint. Overall incidences of treatment-emergent adverse events were similar across groups. There were two deaths on placebo. There were more genital mycotic infections, diarrhea events, and diabetic ketoacidosis in the SOTA arms.

Conclusions: The international inTandem2 trial of SOTA as adjunct therapy to insulin met its primary endpoint with a statistically significant and clinically meaningful $A_{1} C$ reduction after 24 weeks, while demonstrating the general safety and tolerability of dual SGLT1 and $S G L T_{2}$ inhibition in $T_{1} D$. These results confirm the findings of inTandem1.

Study funded by Lexicon Pharmaceuticals, Inc. Editorial support funded by Sanofi. Data presented at the 77th Scientific Sessions of the ADA.

\section{PS103}

Effects of renal denervation on hepatic steatosis and expression of liver RAGE in obese spontaneously hypertensive rats

Juliane Dederer'; Simina-Ramona Selejan ${ }^{2}$; Dominik Linz ${ }^{2}$; Mathias Hohl²; Andrej Kazakov'; Olesja Zamyatkin ${ }^{2}$; Thimoteus Speer ${ }^{2}$; Marcin Krawczyk'; Frank Lammert ${ }^{2}$; Felix Mahfoud ${ }^{2}$; Michael Böhm²

'Universitätsklinikum Homburg, Homburg; ${ }^{2}$ Universitätsklinikum des Saarlandes und Medizinische Fakultät der Universität des Saarlandes, Homburg; ${ }^{2}$ Universitätsklinikum des Saarlandes und Medizinische Fakultät der Universität des Saarlandes, Homburg

Background: The Receptor for Advanced Glycation End products (RAGE) induces oxidative stress, apoptotic reactions and tissue remodeling. Secretory circulating isoforms referred to as soluble RAGE (sRAGE) comprise only the extracellular ligand binding domain and serve as decoy receptors neutralizing RAGE-ligands, having protective roles in non-alcoholic fatty liver disease and preventing such as late diabetic complications. Metabolic syndrome is associated with increased tissue RAGE and RAGE-ligand expression and decreased sRAGE plasma concentrations but also with increased sympathetic activity. Renal sympathetic denervation decreases renal and whole body sympathetic activity and has been shown to provide beneficial off-target effects in a rat model of metabolic syndrome. The effects of sympathetic modulation on liver RAGE/sRAGE balance and hepatic fat accumulation have not been investigated yet.

Methods: Surgical renal denervation (RDN) was performed in a rat model of metabolic syndrome, the Spontaneously Hypertensive Obese Rat (SHROB). SHROB with RDN (SHROB-RDN; $n=5$ ) were compared to sham operated SHROB (SHROB-Sham; $\mathrm{n}=5$ ) and their hypertensive lean controls (SHR; $n=6)$. Animals were sacrificed after 12 weeks and renal norepinephrine was used as indicator of successful RDN. Serum SRAGE, liver RAGE/sRAGE and expression levels of the apoptosis markers $\mathrm{BAX}$ and $\mathrm{BCl}-2$ were assessed by Western blot and immunofluorescence staining. Steatosis was investigated by histological analysis and quantification of ADRP (adipose-differentiation-related-protein, a sensitive marker of lipid accumulation) by immunoblot.

Results: Liver RAGE was significantly increased by $37 \%$ and SRAGE was decreased by $79 \%$ in liver and by $68 \%$ in serum in SHROB when compared with lean littermates SHR. RDN also reduced hepatic RAGE expression by $34 \%$ and increased sRAGE levels by $79 \%$ in liver and $65 \%$ in serum when compared with SHROB-Sham. BAX levels were repressed in SHROB-RDN (-40\%) and $\mathrm{BCl}-2$ was induced (+42\%) versus SHROB-Sham, indicating anti-apoptotic effects of RDN. Steatosis was markedly reduced in SHROB-RDN and liver ADRP contents decreased correspondingly (74\% decrease versus SHROB-Sham).

Conclusions: Renal denervation improves liver sRAGE/RAGE balance, attenuates liver damage and reduces hepatic steatosis in obese 
spontaneously hypertensive rats. These new findings might help to develop new strategies for the treatment of hepatic injury in metabolic syndrome.

\section{PS104 \\ Die Libmann-Sacks Endokarditis ( Fallvorstellung)}

Dorisandra Dumbrava; Hendrik Schmidt; Michael Sudau; Stephan Tetzlaff; Joerg Mittag

Klinikum Magdeburg, Magdeburg

Einleitung: Eine 17-jährige Patientin stellte sich in unserer Notfallambulanz mit linksthorakalen Schmerzen und stattgehabter Synkope vor. Ein Tag vorher hatte sie eine Mumps-Masern-Röteln Impfung erhalten. Anamnestisch Z.n.2-maligen Pneumonien und Z.n.Tonsillitis.

Diagnostik: Laborchemisch zeigten sich eine Troponinerhöhung (1.2 $\mu \mathrm{g} / \mathrm{L}$ ) und eine PTT-Verlängerung ( $62 \mathrm{sec}$ ). Infektparameter unauffällig. Weiterhin Erhöhung von Antinukleären Antikörpern(ANA 1:1600),Lupusinhibitoren sowie Cardiolipin- und/oder ß2-Glycokprotein I-Antikörpern. Die HNO-Untersuchung ergab eine rezidivierende Tonsillitis. Echo:zentrale, flottierende Struktur $(8 \mathrm{~mm})$ an der Aortenklappe mit einer AI I. Kardio-MRT: transmurale Nekrose der basalen und midventrikulären Hinterwand. Blutkulturen: positiv für Streptococcus sanguinis und Staphylococcus epidermidis. CMRT und die ophtalmologische Untersuchung: ohne Anhalt für septische Embolien.

Ergebnisse: Bei V.a. infektiöse Aortenklappenendokarditis erfolgte initial eine Therapie mittels Ampicillin und Gentamycin. Darunter keinen rückläufigen Befund der Vegetation. Die Umstellung auf Ceftriaxon führte zu einem langsamen Rückgang der Vegetation, allerdings entwickelte die Patientin eine toxische Knochenmarkschädigung mit Agranulozytose. Umstellung auf Phenoxymethylpenicillin. Nach 6 Wochen erneute Zunahme der Vegetation. Klinisch Bronchitis mit Fieber. Blutkulturen positiv für Streptococcus gordonii. Es folgte eine Antibiose mittels Meronem nach Resistogramm. Eine erneute Fokussuche (Ganzkörper-MRT) ergab eine Lymphadenopathie (axillär und zervikal) und eine grenzwertige Splenomegalie. Im Zusammenhang der Befunde bestand nun der Verdacht auf systemischen Lupus erythematodes (LSE). Die Patientin wurde in eine rheumatologische Klinik verlegt. Unter Therapie( Hydroxychloroquin und Antikoagulanzien) kam es zu einer raschen klinischen Besserung sowie eine Regredienz der Aortenklappenvegetation.

Schlussfolgerungen: Die Libman-Sacks-Endokarditis ist eine besondere Form der abakteriellen Endokarditis die man beim Krankheitsbild des systemischen Lupus erythematodes in ca. 11\% der Fälle finden kann. Bei etwa 50\% aller durch Lupus-bedingten Todesfälle wurde diese Form der Endokarditis post mortem diagnostiziert. Die Vegetationen sind klein und hämodynamisch unbedeutend. Selten besteht zusätzlich eine Pleuritis oder eine Perikarditis. Das Vorhandensein von Antiphospholipid-Antikörpern erhöht dreifach das Risiko für Endokarditis. Da ihre Diagnose schwierig ist, kommt es relativ häufig zu Komplikationen sowie Klappen- oder Herzinsuffizienz.Die optimale Therapie ist noch nicht bekannt.Im Vordergrund steht die Immunsuppression, deren Vorteil für die LSE ist allerdings bisher nicht eindeutig nachgewiesen. Von chirurgischen Eingriffen wird eher abgeraten. Da unsere Patientin rezidivierend Bakteriämien bei rezidivierender Tonsillitis entwickelte, erfolgte die Differenzierung zwischen bakterieller/abakterieller Endokarditis deutlich erschwert.

\section{PS105}

Auswirkungen der Eisenmangelanämie auf FGF23 bei chronisch nierenkranken Patienten

Insa Emrich'; Johanna Wiedenroth ${ }^{2}$; Kathrin Untersteller'; Simone Lennartz; Sarah Seiler-Mußler'; Danilo Fliser'; Gunnar H. Heine ${ }^{1}$

'Universitätsklinikum des Saarlandes, Homburg, Homburg; ${ }^{2}$ Universität des Saarlandes, Homburg

Chronisch nierenkranke Patienten haben eine hohe kardiovaskuläre Morbidität, zu der ein gestörter Kalzium-Phosphathaushalt beiträgt. Hierbei konnte kürzlich eine Assoziation zwischen den Plasmaspiegeln des phosphaturischen Hormones FGF23 und dem Auftreten von kardiovaskulären Ereignissen beobachtet werden. Unlängst konnte gezeigt werden, dass eine Eisenmangelanämie eine erhöhte Transkription von $\mathrm{FGF}_{23}$ und einen Anstieg von FGF23 Fragmenten im Plasma - welche mit dem c-terminmalen FGF-23 ELISA bestimmt werden -, nicht jedoch von funktionsfähigem (,intakten“) FGF-23 induziert. Wir postulieren nun, dass bei chronisch nierenkranken Menschen mit hohem Plasma FGF-23 die Prädiktion kardiovaskulärer Ereignissen auf einen zugrundeliegenden Eisenmangel und nicht auf eine direkte schädigende Wirkung von FGF-23 zurückgeführt werden kann. In der prospektiven CARE FOR HOMe Studie untersuchten wir daher den Zusammenhang zwischen der Plasma-Ferritin als Reflektor eines Eisenmangels, Plasmaspiegeln von c-terminalem und intaktem FGF 23 und dem Auftreten von kardiovaskulären Ereignissen.

Für die Auswertung standen uns Daten von 567 Patienten zur Verfügung, bei denen Plasma-Ferritin, c-terminales FGF23 und intaktes FGF 23 aus bei Studieneinschluss asservierten Plasmaproben bestimmt wurden. Die teilnehmenden Patienten wurden jährlich im Hinblick auf das Auftreten von (1) atherosklerotische kardiovaskuläre Ereignissen, kardiovaskulären Tod (2) und Tod jedweder Genese (3) nachverfolgt. Sowohl intaktes FGF23 als auch c-terminales $\mathrm{FGF}_{23}$ korrelierten signifikant mit der glomerulären Filtrationsrate $(r=-0.520 ; p<0.001$ vs. $r=-0.598 ; p<0.001)$, während nur C-terminales FGF23 $(r=-0.207 ; p<0.001)$, nicht jedoch intaktes FGF23 mit Plasma- Ferritin korrelierte $(r=-0.016 ; p=0.699)$.

Nach Stratifizierung der Patienten in Tertile war in univariaten Kaplan Meier Analysen Plasma-Ferritin weder mit dem Auftreten von kardiovaskulären Ereignissen noch mit kardiovaskulären Todesfällen noch mit Todesfällen jedweder Genese assoziiert. Auch in den univariaten und multivariaten Cox Regressionsanalysen zeigt sich keine signifikante Assoziation zwischen Ferritin und dem Auftreten der oben genannten Endpunkte.Zusammenfassend zeigte sich erwartungsgemäß ein signifikanter Zusammenhang zwischen c-terminalem $\mathrm{FGF}_{23}$ und Plasma-Ferritin. Ebenso erwartungsgemäß weisen Patienten mit weiter fortgeschrittenen Nierenfunktionseinschränkung und niedrigen Plasma-Ferritin ein deutlich erhöhtes c-terminales FGF23 auf. Allerdings ist entgegen unserer Erwartungen Plasma-Ferritin kein Prädiktor kardiovaskulärer Ereignisse und kann somit die prognostische Bedeutung von FGF-23 nicht erklären. 


\section{PS106}

Fehlende rechte Koronararterie: Ein Kasus von single Koronararterie oder kongenitaler ostialer Atresie - Fallbericht

Richard Eyermann $n^{1,2}$, Helmut Langhof ${ }^{2}$

'Dr. Eyermann, Kinder- und Jugendmedizin, Kinderkardiologie, Sportmedizin, Allgemeinmedizin, München; ${ }^{2}$ Klinik Schönsicht, Rehabilitation für Kinder und Jugendliche, Anschlussheilbehandlung, Kind-Mutter/Vater-Rehabilitation, Berchtesgaden

Problem: Koronararterienanomalien werden in 0,6-1,5\% der Koronarangiogramme detektiert. Eine Atresie des linken Koronararterienostiums ist selten, Atresie des rechten Koronararterienostiums extrem selten $\mathrm{u}$. isoliert, ohne andere Anomalien, bislang erst $1 \mathrm{x}$ in der Literatur beschrieben. Die ungewöhnliche Anomalie wird beschrieben u. anatomische $u$. pathophysiologische Signifikanz u. mögliche Wege der Differenzierung von single Koronararterie diskutiert.

Methode: Fallbericht, evidenzbasierte Literaturrecherche, Praxis-Fazit.

Fallbericht: 30-jährige Frau, mit kardiovaskulären Risikofaktoren Nikotinabusus, Adipositas, familiäre Hyperlipoproteinämie Fredrickson Typ III, arterielle HTN u. positive FA, wurde als Notfall mit V.a. ACS bei akuter Dyspnoe sowie thorakalem Druck mit Ausstrahlung in Halsbereich in einem Herzzentrum stationär aufgenommen. Bereits 3 Tage zuvor konnte in einem Klinikum bei noch stärkeren Beschwerden ein $\mathrm{HI}$ ausgeschlossen werden. Bei fehlenden kardialen Dekompensationszeichen, unauffälligem Ruhe-EKG sowie Echokardiographie (nur LA dilatiert $(44 \mathrm{~mm})$ ), erfolgte bei rezidivierenden AP- Beschwerden mit Dyspnoe zunächst nicht-invasiv zur Ischämiediagnostik ein Belastungs-EKG. Darin zeigten sich zunehmende inferolaterale Erregungsrückbildungsstörungen. Bei ausgeprägtem Risikoprofil folgte die Koronarographie. Angiographisch konnte eine KHK der linken Koronararterien bei zarten Gefäßen ausgeschlossen werden. Trotz wiederholter Darstellungsversuche inkl. Bulbusangiographie war keine rechte Koronararterie darstellbar.

Bei unauffälligem echo- u. levokardiographischem Befund ohne Hinweis auf Myokardnarbe $u$. Nachweis eines proximalen Gefäßanteils bestand V.a. sehr seltene Agenesie der rechten Koronararterie, DD chronisch kollateralisierter Verschluss, DD Abgang rechte Koronararterie aus A. pulmonalis. Im MD-Spiral-CT des Herzens zeigte sich eine Agenesie der RCA (kein älterer Verschluss aus der Aorta), kein Anhalt für atypischen Abgang aus rechter Pulmonalarterie), eine kaliberstarke kräftige LAD, welche Fehlen der RCA kompensiert $u$. nebenbefundlich atypischer Abgang des Ramus diagonalis der CX, unmittelbar zwischenLAD u. CX aus dem Hauptstamm.

Die Patientin wird zunächst konservativ anti-anginös $u$. bezüglich Hyperlipidämie primärprophylaktisch behandelt. In zunächst Unkenntnis des gravierenden Vorbefundes bestehen unter physischer Belastung in der Kind-M/V-Rehabilitation jedoch weiterhin AP-Beschwerden u. zwangen zu Vorsicht gegenüber Gefahr plötzlichen Herztodes u. zu weiterem Vorgehen im Herzzentrum.

Konklusion: Signifikante Koronararterienanomalien sind symptomatisch u. können zu ACS mit $\mathrm{HI}$ u. plötzlichem Herztod bereits in jüngerem Alter führen, bei Adulten v.a. im Kontext mit ausgeprägtem kardiovaskulären Risikoprofil u. dadurch zusätzlichen Koronarflusslimitationen. Atresie rechten Koronararterienostiums ist extrem seltene anatomische Variante der Koronarzirkulation u. schwer von single Koronararterie zu differenzieren.
PS107

Endokarditisprophylaxe bei angeborenen und erworbenen Herzerkrankungen bei Interventionen im Oropharynx - Wissen bei Zahnärzten ( Endocarditis Prophylaxis in Interventions in Oropharynx - Study, EPIO - Studie )

Richard Eyermann $n^{1,2}$

'Dr. Eyermann, Kinder- und Jugendmedizin, Kinderkardiologie, Sportmedizin, Allgemeinmedizin, München; ${ }^{2}$ Klinik Schönsicht, Rehabilitation für Kinder und Jugendliche, Anschlussheilbehandlung, Kind-Mutter/Vater-Rehabilitation, Berchtesgaden

Hintergrund: Die infektiöse Endokarditis(IE) ist mit einer Inzidenz von 7/100.000 Einwohner in Deutschland eine häufige schwere Erkrankung mit nach wie vor hoher Morbidität $u$. Mortalität. Dies belegen Studiendaten u.a. für die AHF des DHZB (CHD-Studie) sowie für die angeborenen und erworbenen Herzerkrankungen im Erwachsenenalter (ALKK-Endokarditis-Register). Die Diagnosestellung IE ist immer wieder mit Schwierigkeiten assoziiert, die Krankenhausmortalität ist $15 \%$ u. erforderlich werden ca. $1 / 3$ akute u. bis $60 \%$ im Intervall chirurgische Interventionen. Zahnärztliche Eingriffe sind eine der Hauptursachen für signifikante Bakteriämien u. führen mit einer Inzidenz von ca. 1.71-2,02 u. Oropharynx-Chirurgie von ca. 2,56/10o Interventionen bei Risikopatienten ohne medikamentöse Prophylaxe(IEP) zu IE, in der Regel innerhalb von 14 Tagen nach dem Eingriff.

Methoden: In Kenntnis der Problematik des noch zu häufigen Unwissens $u$. der mangelnden Akzeptanz der IEP im Zahnärztebereich wurde im Rahmen von bundesweiten Fortbildungen über "Rationale AB-Therapie u. - Prophylaxe in der zahnärztlichen Klinik u. Praxis bei Eingriffen im ZMK-Bereich sowie benachbarter Organsysteme" der Wissensstand bei Zahnärzten vor den Seminaren standardisiert mittels Fragebogen erhoben.

Ergebnis: - 384 Zahnärzte geprüft, 240 m., 144 W., Alter 25-64 J., 23 Kliniker, 361 Praktiker.

- Durchschnitt Wissen um IEP bei dentalen Eingriffen nur ca. 38,6\%, $\min .18,75, \%$ u. $\max .82,82 \%$.

- Kliniker nicht besser als Niedergelassene.

- Kenntnisse v.a. in Indikationen zur IEP, kaum in Praxisumsetzung bei Risikostratifizierung von Patienten.

- Kaum Wissen um leitliniengerechte Präparate u. Dosierungen bei Kindern aber auch Erwachsenen bei Risiko, bei Penizillinunverträglichkeit. Als adäquates $A B$ wird vorrangig Clindamycin genannt, auch bei Penicillinverträglichkeit; deutlich weniger, ca. 1/3, Penicillin V oder Amoxicillin genannt - Aminopenicillin jedoch Mittel 1. Wahl n. Leitlinien bei Patienten ohne Penicillinallergie. Korrekte Dosierung zumeist nur bei Clindamycin bekannt, bei Amoxicillin Dosen von 1ug -10g/die genannt!

Nahezu komplett fehlende Kenntnisse über Dosierungen von $A B$ im Kindes- u. Jugendalter, auch nicht über Maximaldosen.

- Meist Gleichsetzung von IEP u. Therapie dentaler Infektionen: Ca. 2/3 aller Zahnärzte setzen die Prophylaxe fort im Sinne einer Therapie über 3-5 Tage u. länger, i.e. $3 \times 600 \mathrm{mg} /$ die Clindamycin.

- Überversorgung bei fehlender Notwendigkeit einer IEP wie v.a. bei Z. n. PCI (PTCA u. Stentimplantation), Z. n. ACB, Z. n. PM- oder ICD)-Implantation. 
- Ca. 50\% der Zahnärzte erhebt die allgemeine Anamnese nur schriftlich.

Konklusion: Die genaue Datenerfassung des Informationsstandes der Zahnärzte über die medikamentöse Endokarditisprophylaxe bei Risikopatienten mit noch ca. 50\% Unwissen unterstreicht weiterhin den erheblichen Aufklärungsbedarf $u$. die Notwendigkeit von Schulungen zur Akzeptanz der Prophylaxe u. leitliniengerechten Durchführung in dieser ärztlichen Fachgruppe.

\section{PS108 \\ Paradoxe Reaktion auf Salbutamol - Ein Fallbericht signifikanter unerwünschter Nebenwirkung}

Richard Eyermann ${ }^{1,2}$, Helmut Langhof ${ }^{2}$

'Dr. Eyermann, Kinder- und Jugendmedizin, Kinderkardiologie, Sportmedizin, Allgemeinmedizin, München; ${ }^{2}$ Klinik Schönsicht, Rehabilitation für Kinder und Jugendliche, Anschlussheilbehandlung, Kind-Mutter/Vater-Rehabilitation, Berchtesgaden

Problemstellung: Salbutamol wird zur Behandlung von Atemproblemen angewendet, die durch Asthma und COPD (inkl. chronische Bronchitis und Lungenemphysem) verursacht werden. Salbutamol wird außerdem zur Prävention von Asthmabeschwerden angewendet, die durch Belastung, auslösende Reize wie Hausstaub, Pollen, Katzen -oder Hundehaare und Zigarettenrauch verursacht werden. Salbutamol erweitert die Atemwege, lindert die Beschwerden, sollte aber weniger als Dauertherapie regelmäßig verwendet werden. Salbutamol ist zugelassen bei Erwachsenen, Jugendlichen und Kindern im Alter von 4-11 Jahren.

Methodik: Fallbericht, evidenzbasierte Literaturrecherche und Praxis-Fazit.

Ergebnisse: Wie alle Arzneimittel kann auch Salbutamol Nebenwirkungen haben, häufig (bis 1 von 10 Behandelten) Zittern, Tachykardie, Kopfschmerzen und Muskelkrämpfe. Sehr selten (1 von 10.000 Behandelten) können allergische Reaktionen, Blutdruckabfall, Kollaps, erhöhte Aktivität und Reizbarkeit, Halluzinationen, Schlafstörungen, Herzrhythmusstörungen, juckener Hautausschlag und erschwerte Atmung direkt nach Anwendung auftreten.

Berichtet wird über einen 12 Jahre alten Jungen, stationär zur komplexen Adipositasintervention (BMI 27,89kg/m2, BMI-Percentil >9799.5, BMI-SDS 2.24), bei dem erstmalig neben adipositasassoziierter Dyspnoe auch der V.a. Belastungsasthma bestand. In der Lungenfunktion $\mathrm{FEV}_{1} 79 \%$ Soll. Im üblichen Brochospasmolyse-Test kam es nach 2 Hub Salbutamol (DA) zu einer signifikanten anaphylaktischen Schockreaktion mit an der Haut Pruritus und Urticaria, an Lunge und Atemwegen in- und exspiratorischen Stridor und verlängertem Exspirium (Brochospasmus) sowie Tachykardie, Hypotension und Saoz-Abfall $<90 \%$. Unter sofortiger Intensivtherapie mit Unterbrechung der Antigenzufuhr, mit Akutmaßnahmen Gabe von Adrenalin, Volumen, Glukokortikoide, Antihistaminika und 02-Therapie, unter Überwachung der Vitalparameter, konnte das Kind erfolgreich stabilisiert werden. Die unerwünschte Nebenwirkung wurde ordnungsgemäß sowohl der Pharmakovigilanz des Arzneimittelherstellers als auch dem BfArM gemeldet.

Konklusion: Wie alle Arzneimittel kann auch Salbutamol Nebenwirkungen haben, in diesem Fall paradox u.a. zum Bronchospasmus führen. Der anaphylaktische Schock ist eine akut vital bedrohliche Reaktion und erfordert sofortige intensivmedizinische Intervention und Vorgehen nach Klinik bzw. Schweregrad.

\section{PS109}

Seltenes malignes Glomus-caroticum-Paragangliom mit radikaler Resektion und rekonstruktiver Versorgung mittels Hybridprothese als schädelbasisnahes Interponat für die A. carotis interna

Dennis Granowski; Andrej Udelnow; Frank Meyer; Dörthe Jechorek; Christoph Arens; Zuhir Halloul

Universitätsklinikum Magdeburg A.ö.R., Magdeburg

Halstumore sind hinsichtlich ihres adäquaten diagnostischen und therapeutischen Managements anspruchsvoll, vor allem bei malignitätsbedingtem infiltrativen Wachstum, die versorgungsrelevante Gefäße einbeziehen, zur Erreichung eines prognosebestimmenden Ro-Resektionsstatus. Ziel des Fallberichts über den Kasus eines seltenen vaskulär infiltrierenden malignen Glomus-caroticum-Tumors ( $\mathrm{Pa}$ ragangliom) war es, die erfolgreiche interdisziplinär-operative Versorgung durch HNO und Gefäßchirurgie wegen notwendiger operativer Reintervention mit zusätzlicher Gefäßresektion infolge histologisch nachgewiesener Malignität des Erstresektates unter Verwendung einer Hybridprothese für den A.-carotis-interna-Ersatz nahe der Schädelbasis zu präsentieren.

Fall: Ein 38-jähriger Patient wurde wegen eines verschieblichen $\mathrm{Hal}-$ stumors der rechten Seite mittels MRA und DSA diagnostiziert, der Tumor embolisiert und anschließend unter Verwendung eines Propathen-Gefäßersatzes ( $7 \mathrm{~cm}$; W.L. Gore GmbH, Putzbrunn, Deutschland) zwischen $\mathrm{Aa}$. carotis communis et interna operiert - Histologie: malignes Paraganglioma caroticum von $40 \mathrm{~mm}$ Durchmesser mit Haemangiosis et Lymphangiosis carcinomatosa, was zur Re-Op veranlasste (Neck dissection [Level II, III, IV, V] und eine Lymphknotenmetastasen zeigte. Es folgte eine Revision der Gefäßrekonstruktion mit GORE ${ }^{\circledast} \mathrm{Hy}$ brid Vascular Graft Prothesenstent (W.L. Gore GmbH) als Interponat wegen des kurzen extrakraniellen Gefäßstumpfes der distalen A. carotis interna nahe der Schädelbasis und Limitierung der Abklemmzeit. Das frühpostoperative Outcome war komplikationslos, nach 12 Monaten besteht kein Anhalt für ein Tumorrezidiv.

Schlussfolgerung: Ausgedehnte Resektionen, notfalls auch unter Mitnahme von (arteriellen) Gefäßsegmenten dienen der Erreichung einer onkologischen Ro-Situation wie bei dieser extrem seltenen und anspruchsvollen Tumorentität. Hybridprothesen ermöglichen die zeitsparende Gefäßrekonstruktion und die Minderung von Ischämiezeiten.

\section{PS110}

Lungenentblähung mit Indacaterol/Glycopyrronium verbessert die kardiale Funktion von COPD Patienten: Die CLAIM Studie

Jens M Hohlfeld'; Jens Vogel-Claussen ${ }^{2}$; Heike Biller'; Dominik Berliner²; Korbinian Berschneider ${ }^{3}$; Hanns-Christian Tillmann ${ }^{4}$; Simone Hiltt ${ }^{3}$; Johann Bauersachs ${ }^{2}$; Tobias Welte ${ }^{2}$

'Fraunhofer Institut für Toxikologie und Experimentelle Medizin, Hannover; ${ }^{2}$ Medizinische Hochschule Hannover, Hannover ${ }^{3}$ Novartis Pharma GmbH, Nürnberg; ${ }^{4}$ Novartis Institutes for Biomedical Research, Basel, Switzerland;

Einleitung: Kardiovaskuläre Erkrankungen sind bedeutende Komorbiditäten der COPD mit einem signifikanten Einfluss auf Morbidität und Mortalität. Dabei ist insbesondere Lungenüberblähung mit verminderten kardialen Volumina und reduziertem Herzzeitvolumen assoziiert. Die CLAIM Studie untersucht den Einfluss der Entblähung der Lunge mit dem dualen Bronchodilatator Indacaterol/Glycopyrronium (IND/GLY) auf die kardiale Funktion bei COPD Patienten mit erhöhtem Residualvolumen. 
Methoden: CLAIM ist eine monozentrische, randomisierte, doppelt-blinde, Placebo-kontrollierte cross-over Studie. COPD Patienten mit erhöhtem Residualvolumen und ohne klinisch signifikante kardiovaskuläre Erkrankungen wurden auf eine von zwei möglichen Behandlungssequenzen (IND/GLY 110/50 $\mu$ g inhalativ q.d. gefolgt von Placebo oder umgekehrt) randomisiert. Primärer Endpunkt war die Veränderung im linksventrikulären enddiastolischen Volumen (LVEDV) gemessen mittels MRI nach 14-tägiger Behandlung.

Ergebnisse: Von 62 randomisierten Patienten mit symptomatischer COPD und erhöhtem Residualvolumen durchliefen 57 beide Behandlungsphasen. IND/GLY führte zu einer klinisch relevanten und statistisch signifikanten Verbesserung der Lungenfunktion und des Residualvolumens (-0.75 L [95\% Cl: -0,93 bis -0,58; $\mathrm{p}$

\section{PS111 \\ Fallbericht: Anhaltende Diarrhoe einer älteren Dame - Wie Blut- druck und Diarrhoe zusammengehören.}

Hanna Israel; Uwe Wahl

\section{BG-Kliniken Bergmannstrost, Halle}

Wir berichten von einer 72-jährigen Patientin mit therapieresistenter Diarrhoe einhergehend mit Verschlechterung des Allgemeinzustands und Exsikkose. Bei negativem Keimnachweis erfolgte die weiterführende endoskopische Diagnostik. Makroskopisch erwies sich sowohl Dünn-, als auch Dickdarm als unauffällig. Histologisch zeigte sich im Duodenum eine deutliche Zottenatrophie und Kryptenhyperplasie im Sinne einer Zöliakie. Die Histologie des Kolons erbrachte den Befund einer lymphozytären Kolitis. Bei negativem Antikörpernachweis für eine glutensensitve Enteropathie erfolgte die Untersuchung des Nebenwirkungsprofils der bestehenden Hausmedikation. Für den Angiotensin-II-Antagonsiten Olmesartan ist in seltenen Fällen (unter $0,01 \%$ ) das Auftreten einer spruähnlichen glutensensitiven Enteropathie beschrieben. Diese Nebenwirkung tritt im Schnitt drei Jahre nach Beginn der Einnahme auf. Nach Absetzen des Antihypertensivums kam es im Verlauf zu einer raschen Besserung der Symptomatik. Obwohl der genaue Mechanismus der sartaninduzierten Enteropathie nicht vollständig geklärt ist, werden Mechanismen mit Beteiligung von IL-15 und TGF- $\beta$ diskutiert. Wichtige Unterschiede zur normalen Zöliakie sind in der negative Antikörpernachweis, die fehlende Besserung durch glutenfreie Ernährung und die Besserung nach Absetzen des verursachenden Medikaments. Glutenhaltige Präparate können komplikationslos verzehrt werden. Die Patientin zeigte nach Absetzen des Olmesartans eine zügige Besserung der beschriebenen Symptomatik. Eine Kontrollgastroskopie nach $1 / 2$ Jahr Medikamentkarenz zeigte einen unauffälligen histologischen Befund. Medikamente können als Nebenwirkungsprofil Durchfälle und Bauchkrämpfe verursachen. Häufig findet man in der endoskopischen Diagnostik das histologische Bild der mikroskopischen Kolitis einerseits und das Bild einer Zöliakie andererseits. Sind zöliakietypische Antikörper nicht nachweisbar, muss an einen medikamenteninduzierten Pathomechanismus denken. Besonders betroffen sind ältere Patientinnen mit anhaltender Diarrhoe ohne infektiöse Ursache. Eine Diagnosis ex juvantibus kann eine aufwendige und möglicherweise teure Differentialdiagnostik bei anamnestischen und klinischen Hinweisen verzichtbar machen.

\section{Interdisziplinarität in der Inneren Medizin II}

\section{PS112 \\ Kardialer Befall durch Aggressives Lymphom. Eine internistische interdisziplinäre Seltenheit}

Madalina Ivan; Thomas Okech; Cornelius Ehmann; Thushira Weerawarna; Manfred Krammer; Paul Weber

Siloah St. Trudpert Klinikum, Pforzheim

Das diffuse großzellige B-Zell-Lymphom ist die häufigste Neoplasie des lymphatischen Systems und ist charakterisiert durch rasch progrediente Lymphknotenvergrößerungen/extranodale Manifestationen. ES führt unbehandelt rasch zum Tod. Ein kardialer Befall im hohen Alter erfordert in der Diagnostik und Therapieauswahl einen individualisierten Ansatz. Die stationäre Aufnahme unseres Patienten (78 J) erfolgte wegen Hämoptysen, Belastungsdyspnoe und wochenlangem therapierefraktärem Husten. Die körperliche Untersuchung ergab außer einem großen rechtsseitigen Pleuraerguss bei reduziertem Allgemeinzustand keine Besonderheiten. Die Echokardiographie zeigte eine Raumforderung im rechten Vorhof mit Übergreifen in den linken Vorhof. Im TEE zeigte sich eine den rechten Vorhof komprimierende Tumormasse, die die Vorhöfe nach links verlagerte und einen flottierenden Thrombus im rechten Vorhof. Im CT des Thorax bestätigte sich eine ausgedehnte tumoröse Raumforderung des oberen Mediastinums mit Einwachsen in den rechten Vorhof über die V. cava superior, Infiltration des Vorhofseptums, Kompression der Lungenvenen und Ummauerung des rechten Mittellappenbronchus.Das Vorhofmyokard war nicht abgrenzbar. Eine intrakardiale Probeentnahme einer Biopsiezange wäre technisch machbar gewesen, aber als zu risikoreich bewertet worden. Bronchoskopisch war kein exophytischer Tumor sichtbar. Die transbronchiale Nadelaspiration TBNA der vergrößerten subkarinalen Lymphknoten ergab einen Verdacht auf ein hoch malignes Lymphoms. Wegen ausgedehnter Nekrosen konnte jedoch das Lymphom nicht subklassifiziert werden. Interventionell wurde mittels der starren Bronchoskopie Proben gewonnen. Die Histologie ergab wiederum nekrotisches Material sowie CD 45-positive Zellen ohne Expression von Melan A, aber starke CD 20 Positivität. Die Pleurapunktion mit patho-histochemischen Nachweis von Zellen mit kräftiger CD 20 Expression, BCL2-Protein und BCL6 bei fehlender Expression von $\mathrm{CD} 30, \mathrm{CD} 138, \mathrm{CD}_{3}$ und c-myc erbrachte die Diagnose eines malignen diffusen großzelligen B-Zell-Lymphom (NOS). Ein Hodgkin-Lymphom konnte ausgeschlossen. Die Erstlinientherapie erfolgte mit 6 Zyklen CHOP und 8 Gaben Rituximab. Wir haben unserem Patienten einer Dosis Immunochemotherapie nach R-mini-CHOP unter kontinuierlicher Antikoagulation verabreicht. Diese wurde gut vertragen und zeigte nach 3 Wochen eine fast vollständige Remission. Der Therapieanspruch ist kurativ so dass wir eine Therapie Eskalation bei guter Verträglichkeit erwägen.

Fazit: Die Initial Symptomatik, die Lokalisation dieser seltenen Krankheit, der diagnostische Weg sowie die Therapie erforderte eine interdisziplinäre Vorgehensweise und eröffnete dem Patienten so eine therapeutische Option mit einer kurativen Perspektive. 


\section{PS113}

\section{Späte Phänotypisierung einer apikalen Form der hypertrophen Kardiomyopathie}

Angelika Koljaja-Batzner'; Barbara Pfeiffer'; Hubert Seggewiss²

'Leopoldina-Krankenhaus der Stadt Schweinfurt GmbH, Schweinfurt;

${ }^{2}$ Praxis, Schweinfurt

Einleitung: Die hypertrophe Kardiomyopathie (HCM) ist eine häufige genetische myokardiale Erkrankung. Hierbei ist der Phänoptyp und die Ausprägung der Symptome sehr variabel und ein Auftreten noch im fortgeschritteneren Alter möglich. Im Folgenden soll der Verlauf einer initial nicht apparenten apikalen HCM dargestellt werden.

Fallbericht: Der 43-jährige Patient wurde erstmalig 2001 wegen akut aufgetretener pectanginöser Beschwerden in Ruhe vorstellig. Bei kardiovaskulärem Risikoprofil (arterielle Hypertonie, Hypercholesterinämie, Nikotinabusus) und typischer Symptomatik entschloss man sich zu einer Herzkatheteruntersuchung, wobei ein Ausschluss einer stenosierenden KHK und Nachweis einer Muskelbrücke in der mittleren LAD erfolgte. Im Ruhe-EKG fanden sich keine Auffälligkeiten, im transthorakalen Echokardiogramm eine grenzwertig verdickte Hinterwand bei schlechter Schallbarkeit. 11 Jahre später wird der Patient erneut wegen retrosternaler Schmerzen, jetzt auch von Dyspnoe begleitet, vorstellig. Im Ruhe EKG fanden sich nun neue ausgeprägte T-Negativierungen in I und aVL sowie über der gesamten Vorderwand und ein grenzwertig breiter QRS Komplex ohne das Bild eines Linksschenkelblocks. Darüber hinaus war sah man Zeichen einer linksventrikulären Hypertrophie mit einem Sokolow-Lyon-Index von 3,8 mV. Im Rhythmusscreening fanden sich wenige polymorphe ventrikuläre Extrasytolen. Echokardiographisch fand sich zunächst bei schlechten Schallfenstern keine linksventrikuläre Wandhypertrophie und eine Dilatation des linken Vorhofs von $45 \mathrm{~mm}$. In der erneut durchgeführten Herzkatheteruntersuchung zeigte sich neben einer Koronarsklerose in der LV-Angiographie eine apikale HCM mit typischer Pik-AssForm. Die anschließende Kontrastechokardiographie bestätigte den Befund der apikalen HCM mit einer apikalen Wanddicke von $20 \mathrm{~mm}$.

Beim erweiterten Rhythmusscreening in häuslicher Umgebung 2 Wochen später fanden sich neben intermittierendem Vorhofflimmern auch nsVTs (5 konsekutive Schläge mit einer Frequenz von 202 / Min). Nach dem aktuellen Risiko Score der Europäischen Kardiologengesellschaft betrug das 5-Jahresrisiko des plötzlichen Herztod 4,1\%. Daraufhin erfolgte eine erneute stationäre Aufnahme zur Diskussion einer primärprophylaktischen ICD-Implantation. Nach Erhöhung der Betablockerdosis fiel eine nächtliche Sinusbradykardie auf bei jetzt nicht mehr nachweisbaren Tachykardien. Einer ICD-Implantation stimmte der Patient nicht zu. Ein Termin zur erneuten Rhythmuskontrolle wurde vom Patienten nicht wahrgenommen. 10 Monate später kam der Patient unter laufender Reanimation in die Klinik. Er verstarb am plötzlichen Herztod.

Zusammenfassung: Der Verlauf bei diesem Fall unterstreicht die Wichtigkeit von wiederholten Untersuchungen bei typischer kardialer Symptomatik. Der Phänotyp einer HCM kann auch in höherem Lebensalter auftreten. Bei Patienten mit HCM sind regelmäßige Risikostratifizierungen bezüglich des plötzlichen Herztodes unabdingbar.

\section{PS114}

Carotido-subclavialer Bypass bei A.-subclavia-Stenose und seltener Kombination von ,Subclavian-Steal'-Syndrom mit supraaortaler Gefäßabgangsanomalie (Fallbericht)

\author{
Kristina Korsake'; Björn Friebe ${ }^{2}$; Torsten Mildner ; Frank Meyer ${ }^{2}$ \\ ${ }^{1}$ Harzklinikum „Dorothea Erxleben“, Wernigerode, Wernigerode; ${ }^{2} \mathrm{Ot}-$ \\ to-von-Guericke-Universität mit Universitätsklinikum Magdeburg, \\ Magdeburg
}

Hintergrund: Das "Subclavian-Steal"-Syndrom (SSS) ist ein vaskuläres Phänomen, bei dem durch eine hochgradige Stenose oder einen Verschluss der proximalen A. subclavia ein retrograder Fluss in der A. vertebralis besteht und die Symptome einer vertebrobasilaren Insuffizienz oder peripher-zerebralen Minderversorgung auftreten.

Fallkonstellation: Die stationäre Aufnahme einer 69-jährigen Patientin erfolgte durch ambulanten Angiologen auf Grund von Schwindel, Schwanken und präsynkopaler Zustände, insbesondere bei Bewegung des linken Armes bei einer Über-Kopf-Arbeit und aufgetretenen Sensibilitätstörungen links-temporal. Es bestand eine Bludruckdifferenz von $40 \mathrm{mmHg}$ zugunsten des rechten Armes.

Das Angio-MRT zeigte folgende Anomalie der supraaortalen Gefäßabgänge: Rechts deszendierender Aortenbogen mit proximal nebeneinander aus dem Aortenbogen entspringenden A. carotis communis sinistra (ohne Stenosen) und A. subclavia sinistra mit 2,5-cm-Okkusion im Ursprungssegment bei offenem Abgang der A. vertebralis sinistra mit arterieller Versorgung des linken Arms über einen retrograden Fluss via A. vertebralis sinistra. A. carotis communis dextra sowie die A. subclavia dextra mit daraus abgehender $A$. vertebralis dextra zeigen einen unauffälligen Verlauf.

Methode: Wegen fehlender Langzeitergebnisse einer endovaskulären Therapie, unklarer Rekanalisationsaussicht des A.-subclavia-sinistra-Segments sowie der Gefäßanomalie und in Anbetracht einer provozierbaren, tendenziell zunehmenden Symptomatik erfolgte die Anlage eines carotido-subclavialen Prothesenbypasses links zwischen den Aa. carotis communis et subclavia sinistrae mittels 6-mm-beringter PROPATEN ${ }^{\circledR}$-Prothese. Der klinisch-neurologische Status und die neurovaskuläre Dopplersonographie postoperativ befundeten einen offenen Bypass. Die A. vertebralis sinistra war noch mit inkomplettem Stealeffekt (Pendelfluss) ableitbar, die A. basilaris noch mit geringem "Prästeal"-Effekt. Jährliche ambulante Kontrollen zeigen mittlerweile schon 7 Jahre lang ein stabiles Ergebnis, wie anfangs beschrieben und ohne eruierbare klinische Symptomatik.

Ergebnis: Das symptomatische SSS ist ein therapiewürdiger Befund mit absoluter Indikation. Heutige Therapieempfehlungen tendieren zur interventionell-radiologischen Versorgung als Therapie der ersten Wahl, jedoch mit sich erst etablierenden suffizienten Langzeiterfahrungen. Chirurgische Verfahren zeigen hingegen ein gesichertes und ansprechendes Langzeit-Outcome und sind im präsentierten Fall daher als absolut indizierte und prognostisch aussichtsreichste Vorgehensweise anzusehen. 


\section{PS115}

Interimsanalyse zu konsekutiven EUS-geführte Enteroanastomosen bei malignitätsbedingter Cholestase und Afferent-Loop-Syndrom (u.a. im Zusammenhang mit Pancreas-, Papillen- und periampullären Malignomen) unter Nutzung des AXIOS-StentsTM

Viliam Masaryk ${ }^{1}$; Frank Füldner'; Frank Meyer ${ }^{2}$; Uwe Will'

${ }^{1} \mathrm{SRH}$ Wald-Klinikum Gera GmbH, Gera; ${ }^{2}$ Universitätsklinikum Magdeburg A.ö.R., Magdeburg

Ziel \& Methodik: Mittels klin.-systemat. prospektiver Datenerfassung im Rahmen einer unizentr. Observationsstudie an einer konsekutiven Pat.-Kohorte eines Zentrums der interventionellen GI-Endoskopie/-Endosonographie(EUS) über einen def. Zeitraum sollten in einer Interimsanalyse techn. Machbarkeit, Outcome (techn./klin. Erfolgsrate), periinterventionelle Morbidität (insbes. Spektrum \& Rate von Früh- \& Spätkomplikationen incl. ihrer interventionell-endoskop. Angehbarkeit)/Letalität von EUS-geführten Enteroanastomosen u.a. bei Pancreas-, Papillen- \& periamp. Malignomen nicht zuletzt zur internen Qual.-Sicherung eruiert werden. Vorgehenstechnisch erfolgte eine EUS-geführte Gastro-Jejunostomie mittels transgastraler(-lumenaler) Darstellung der gestauten zuführenden Schlinge, Durchbrennen der Sonde, Platzierung eines Führungsdrahtes (Vermeiden des Vorbeigleitens des AXIOS-StentsTM an der Darmwand), die Freisetzung des AXIOS-StentsTM \& die Herstellung der Anastomose in Sekunden (Folgetag: Sonographiekontrolle zur Dok. von entstauter zuführender Schlinge \& die korrekte Platzierung des AXIOS-StentsTM in situ \& Ausschluss unkl. Flüssigkeitsansammlung, freier Luft etc.).

Ergebnisse: Von 2013-2017 wurden 13 Pat. im Alter von 43-85 Jahren therapiert. Es wurden überwiegend Gastrojejunostomien $(n=11 / 84,6 \%)$ neben einer Duodeno-Jejunostomie \& einer Jejuno-Jejunostomie (je 7,7\%) mit Hot-AXIOS-StentTM angelegt. Technisch war die AXIOS-StentTM-Anlage bei 12/13 Patienten erfolgreich (92\%). Es traten 2 Anlagekomplikationen auf $(15,4 \%)$ :

- Bei 1 Pat. kam es wg. schwerer EUS-basierter Einstellbarkeit zu einer Fehllage des Stents - der AXIOS-StentTM wurde entfernt \& die entstandene Perforation der Magenwand mit OTSC verschlossen. Die Drainage erfolgte daraufhin endosonographisch über eine translumenale Hepatico-Gastrostomie.

- Beim 2. Pat. war ein 2. AXIOS-StentTM wegen init. Fehllage notwendig.

Klin. Erfolg mit Regress der Cholestase war bei allen Pat. zu verzeichnen (Rate: 100\%).

Im „Follow-up“ waren bei 2 Pat. Reinterventionen wg. Stentokklusion \& Einwachsen des Stents in die Magenwand, auch Buried-Stent-Syndrom genannt, erforderlich. Durch Ballondilatation, Anlage von Plastprothesen \& im Verlauf eines Metallstents konnte bei beiden Patienten eine Drainage gesichert bzw. eine GI-Passage wieder hergestellt werden. Eine stentbedingte Blutung oder Infektion wurde nicht beobachtet. Eine viszeralchirurgische Revision war in keinem Fall erforderlich. Eine Intervention über den AXIOS-StentTM ermöglichte im postinterventionellen Verlauf die min.-invasive, interventionell-endoskop. geführte intrahepat. Steinextraktion \& Entfernung eines fehlliegenden inkrustierten biliären Stents.

Schlussfolgerung: Durch Vermeidung eines op. Eingriffes oder transkutaner Drainage stellt die GE mit AXIOS-StentTM als vielversprechende $\&$ techn. machbare als auch sichere Methode bei Afferent-loop-Syndrom eine hoffnungsvolle Aussicht auf eine zügige Rekonvaleszenz \& Verbesserung der Lebensqualität dar.
PS116

Cholemic nephropathy is frequently observed in jaundiced patients and associated with increased mortality

Young-Seon Mederacke; Jessica Schmitz; Roland Schmitt; Michael Peter Manns; Jan Hinrich Bräsen; Ingmar Mederacke

Medizinische Hochschule Hannover, Hannover

Background and Aims: Impairment of renal function often occurs in patients with liver disease and is associated with an increased mortality. Hepatorenal syndrome is a significant cause of acute kidney injury (AKI) in cirrhotic patients (HRS-AKI, type 1), however, a kidney biopsy to exclude structural causes of kidney disease is rarely performed owing to increased risk of bleeding in this cohort. Causes of non-HRS AKI include cholemic nephropathy $(\mathrm{CN})$, a disease that has regained attention recently and that is characterized by intratubular cast and tubular injury. As data on patients with $\mathrm{CN}$ is mostly obtained from case reports or autopsy studies, we aimed to investigate the prevalence and clinical course of $\mathrm{CN}$ in a tertiary care hospital over a 16-year period.

Methods: We identified 149 patients who underwent kidney biopsy from 2000 to 2016 at the Department of Gastroenterology, Hepatology and Endocrinology. Of these 79 had a history of liver disease and deterioration of renal function and were included into this study. Laboratory values and clinical course of the patients were obtained by retrospective chart analysis. Biopsies were reevaluated to confirm the diagnosis of $\mathrm{CN}$.

Results: The mean age of the patients was $52.2+/-\quad 10.8$ years and $71 \%$ were male patients $(56 / 79)$. One quarter of the patients had a history of organ transplantation (liver or kidney), cirrhosis detected by liver biopsy or ultrasound was observed in 43 of 79 patients (54.4\%). The average creatinine at the time of biopsy was $256+/-181 \mu \mathrm{mol} / \mathrm{l}$. Consistent with the increased risk of bleeding in patients with advanced liver disease 25 patients had an INR $>2$ or platelet count $<100.000 /$ $\mu$ l. Biopsy related complications occurred in six of 79 patients, one of them required surgical therapy. Renal biopsy revealed the diagnosis of $\mathrm{CN}$ in $10.1 \%$ of the patients (8/79). Patients with $\mathrm{CN}$ presented with significantly higher bilirubin ( $779+/-306 \mathrm{vs.} 66+/-110 \mu \mathrm{mol} / \mathrm{l}, \mathrm{p}$ $<0.001$ ), whereas creatinine, ALT, AST, INR, liver function and blood count were similar in both groups. Of note, patients with $\mathrm{CN}$ secreted bilirubin (100\%) and urobilirubinogen (50\%) in the urine, compared to $15 \%$ and 13 of the non-CN patients, respectively $(p<0.05)$. Most importantly, diagnosis of $\mathrm{CN}$ was linked with increased mortality $(p<$ 0.05).

Conclusion: Cholemic nephropathy is associated with increased mortality and should be considered as a cause of impaired renal function in patients with liver disease and elevated bilirubin levels.

\section{PS117}

Patient mit einer pankreatischen Pannikulitis - eine dermatologische Erkrankung oder eine internistische Pankreaserkrankung?

Patricia-Iuliana Mester; Georg Peschel; Kilian Weigand; Martina Müller

Universitätsklinikum Regensburg, Regensburg

Hintergrund: Die pankreatische Pannikulitis ist eine seltene Erkrankung geprägt von disseminierten Fettgewebsnekrosen, die bei 2-3\% aller Patienten mit Pankreaserkrankungen auftritt. Der Zusammenhang zwischen einer chronischen oder akuten Erkrankung der Bauchspeicheldrüse und entfernten Fettnekrosen des subkutanen 
Fettgewebes wurde erstmalig von Chiari in 1883 beschrieben. Pathophysiologisch sind fokale enzymatische Wirkungen der vom Pankreas freigesetzten Lipasen oder der alpha-Amylase anzunehmen.

Klinischer Fall: Wir berichten über den Fall einer schweren pankreatischen Pannikulitis ohne Hinweise auf einen akuten Schub oder einer chronischen Pankreatitis bei einer 66-jährigen Patientin. Die Patientin hatte sich in unserer interdisziplinären Notaufnahme wegen seit 2 Wochen progredienter roter, knotiger schmerzhafter Hautveränderungen an beiden Unterschenkeln vorgestellt.

Methode: Laborchemisch zeigten sich erhöhte Entzündungswerte sowie eine Lipase von $11130 \mathrm{U} / \mathrm{l}$. Eine systemische Stoßtherapie mit Prednisolon erbrachte keine Besserung. Die Patientin wurde in die Abteilung Innere Medizin I der Universitätsklinikum Regensburg bei Verdacht auf einer akuten Pankreatitis stationär aufgenommen. Es erfolgte eine tiefe Biopsie im Bereich der palpablen Nodi am Unterschenkel. Hierbei zeigte sich den Befund vereinbar mit einer pankreatischen Pannikulitis. Die Endosonographie zeigte keine eindeutigen Zeichen einer chronischen Pankreatitis. Eine lgG4-assoziierte-Pankreatitis konnte ausgeschlossen werden. Eine FDG-PET-CT-Untersuchung erbrachte weder einen Anhalt für Lipase produzierende Tumoren noch für eine Vasculitis. Die Entzündungswerte waren im Verlauf rückläufig. Deswegen gehen wir von einer Immunaktivierung im Rahmen der dermatologischen Erkrankung aus.

Diskussion und Schlussfolgerung: Die Pathogenese dieser Krankheit ist nicht komplett bekannt. Man vermutet, dass pankreatische Enzyme wie Trypsin die Permeabilität und die Mikrozirkulation steigern. Dadurch werden Fette abgebaut und es kommt zur Inflammation und zu einer gestörten Immunreaktion im subkutanen Fettgewebe. Differentialdiagnostisch muss man das Erythema Nodosum, das subkutane Pannikulitis-artige T-Zell-Lymphom oder die Alpha-1-Antitrypsin Deficiency Pannikulitis bedenken, da das makroskopische Bild unspezifisch ist. Anhand der Hautbiopsie wird die endgültige Diagnose gestellt. Die weitere Behandlung bezieht sich auf die Therapie der Grunderkrankung. Bei unserer Patientin ergaben sich keine Hinweise auf eine aktive Pankreaserkrankung. Die pankreatische Pannikulitis ist pathognomonisch für eine Pankreaserkrankung und wie in unserem Fall können manchmal die Hautläsionen der zugrundliegenden Erkrankung um Wochen oder Monaten vorgreifen. Zusammenfassend sollte man die pankreatische Pannikulitis als eine mögliche Manifestation einer pankreatischen Krankheit in Betracht ziehen.

\section{PS119 \\ Partielle Milzembolisation als Rescue-Behandlungsoption bei Varizen}

Vlad Pavel; Holger Gößmann; Claudia Zimmermann; Martina Müller; Stephan Schmid

Universitätsklinikum Regensburg, Regensburg

Hintergrund: Die partielle Milzarterienembolisation ist ein nicht-chirurgisches Verfahren, welches zunächst für die Behandlung des primären und sekundären Hypersplenismus etabliert wurde. Bereits im Jahre 1973 erfolgte durch Maddison die erste Milzembolisation bei Hypersplenismus mittels eines Blutkoagels. Heute wird die Milzarterienembolisation bei verschiedenen Erkrankungen, einschließlich der Rescue- Behandlung von Ösophagus- und Fundusvarizen bei portaler Hypertension und/oder Milzvenenthrombose angewandt. Weiterhin ist sie eine sehr gute Alternative zur Splenektomie. In vielen Studien konnte eine signifikante Reduktion der Häufigkeit von Varizenblutungen durch eine Milzarterienembolisation nachgewiesen werden. Zudem kommt es postinterventionell oft zu einer Verbesserung der Leberfunktion.
Klinische Fälle: Sechs Patienten mit Ösophagusvarizen Grad IIIIV- und Fundusvarizen die mittels Milzarterienembolisation als Rescue-Therapie behandelt wurde, werden dargestellt. Endoskopische Therapieoptionen waren aufgrund von Fundusvarizen nicht erfolgsversprechend. Auch die Anlage eines transjugulären intrahepatischen portosystemischen Shunts (TIPS) war auf Grund der Anatomie nicht möglich. Die Interventionen wurden innerhalb eines Jahres am Universitätsklinikum Regensburg durch das Institut für Röntgendiagnostik durchgeführt. Alle Patienten hatten aufgrund verschiedener Grunderkrankungen eine ausgeprägte portale Hypertension: Faktor V Leiden, JAK2 Mutation, Primär sklerosierende Cholangitis, Polycythemia vera. Bei allen sechs Patienten wurde die Milzarterienembolisation erfolgreich durchgeführt. In der anschließenden Gastroskopie zeigte sich eine signifikante Abnahme der Ösophagus- und Fundusvarizen, welche dann als Grad II oder niedriger eingestuft wurden. Bei keinem Patienten kam es in der Zeit nach der Intervention zu einer varikösen Blutung. Laborchemisch kam es stets zu einer Verbesserung. Schwere Komplikationen wurden nicht beobachtet.

Diskussion und Schlussfolgerung: Bei Patienten mit portaler Hypertension und konventionell nicht beherrschbaren Ösophagus- und Fundusvarizen kommt es nach Milzarterienembolisation zu einer signifikante Reduktion der Blutungsereignisse (Reduktion von Varizenblutungen um $80 \% / \mathrm{Jahr}$ ) sowie zu einer anhaltenden klinischen und laborchemischen Verbesserung der Leberfunktion. Durch Verbesserung der verfügbaren Technologien und des interventionellen Vorgehens konnten die Komplikationsrate deutlich reduziert werden. Wie in unseren klinischen Fällen gezeigt, ist die Milzarterienembolisation eine gute Therapieoption für Patienten mit Ösophagus- und Fundusvarizen, welche endoskopisch und mittels TIPS nicht beherrschbar sind. Bei allen sechs Patienten zeigte sich ein sehr guter Therapieerfolg sowie eine hervorragende Lebensqualität.

\section{PS120 \\ Reversible Proteinurie nephrotischen Grades bei funktioneller Einnierigkeit, Nierenarterienstenose und IgA-Nephritis}

Jan Schiefer'; Hermann-Josef Gröne²; Peter Mertens'; Christos Chatzikyrkou'

${ }^{1}$ Universitätsklinikum Magdeburg, Magdeburg; ${ }^{2}$ Deutsches Krebsforschungszentrum Heidelberg, Heidelberg

Fallvorstellung: Bei der Vorstellung eines 61-jährigen Patienten zur Abklärung einer Niereninsuffizienz (eGFR $35 \mathrm{ml} / \mathrm{min}$ ) mit unselektiver großer Proteinurie $(2,1 \mathrm{~g} / \mathrm{g}$ Kreatinin) war unter einer 5-fach antihypertensiven Medikation weiterhin eine Hypertonie (ED vor 20 Jahren, Durchschnitt $176 / 87 \mathrm{mmHg}$ in der 24h-RR-Messung) nachweisbar. Sonographisch war die rechte Niere unauffällig, links präsentierte sich eine zystisch degenerierte Niere. Die antihypertensive Therapie wurde intensiviert, eine Nierenbiopsie lehnte der Patient ab. 6 Monate später zeigte sich das komplette Bild eines nephrotischen Syndroms (Proteinurie 17,0 g/g Kreatinin mit Anasarka) sowie eine weitere Abnahme der Nierenfunktion (eGFR $22 \mathrm{ml} / \mathrm{min}$ ). Nach Besserung der Blutdruckeinstellung erfolgte nach Einwilligung des Patienten eine Nierenbiopsie rechts mit typischen Befunden der Triple-Diagnostik bestehend aus Lichtmikroskopie, Immunhistochemie und Elektronenmikroskopie. Zum einen zeigte sich eine IgA-Nephritis mit Hyperzellularität, Matrixvermehrung sowie IgA- und $\mathrm{C}_{3}$-Ablagerungen mesangial. Zum anderen präsentierte sich eine fokal-segmentale, fokal-globale Glomerulosklerose (verödete Kapillaren mit Fibrinnachweis, Schaumzellen in den Kapillaren) und ein fortgeschrittener tubulointerstiteller Schaden mit interstitieller Matrixvermehrung, mononukleärer Infiltration und Tubulusatrophie (Abb. 1a-c). Bei akuter Verschlechterung der eGFR unter ACE-Hemmer-Gabe sowie duplexsonographisch nachweisbarer abgangsnaher hämodynamisch relevanter Nierenarterienstenose rechts (Vmax $688 \mathrm{~cm} / \mathrm{s}$, Abb. 2) 
bei funktioneller Einzelniere wurde eine PTA mit Stent-Implantation durchgeführt (Abb. 3a). Postinterventionell kam es zur Polyurie. Nierenfunktion und Proteinurie besserten sich (eGFR $24 \mathrm{ml} / \mathrm{min}$, Abfall Proteinurie auf 2,4 g/g Kreatinin am Tag 4 nach PTA; $49 \mathrm{ml} / \mathrm{min}$ und $1,8 \mathrm{~g} / \mathrm{g}$ Kreatinin nach 3 Monaten). Die antihypertensive Therapie konnte deeskaliert werden. Duplexsonographisch wurde eine Normalisierung des Strömungsprofils nachgewiesen (Abb. 3b). Bei zuletzt persistierender Proteinurie subnephrotischen Grades wurde für die IgA-Nephritis retardiertes Budesonid verordnet, zudem war nach der PTA eine RAAS-Blockade möglich.

Schlussfolgerungen: Proteinurie und GFR-Verlust können multifaktoriell bedingt sein. Es lohnt in unklaren Fällen eine Nierenbiopsie, zudem ist die Perfusion der Nieren zu überprüfen. Die ischämische Nephropathie geht mit progredientem GFR-Verlust und oft refraktärer Hypertonie einher, ein nephrotisches Syndrom ist sehr untypisch. Die Reversibilität der Proteinurie nach PTA weist auf eine Kausalität hin, der genaue Mechanismus ist jedoch unklar. Multiple Pathologien können multiple Therapien erfordern, wie in unserem Fall die PTA der Nierenarterienstenose und Budesonid zur Modulation des enteralen Immunsystems, welches in der Pathogenese der IgA-Nephritis eine zentrale Rolle spielt.

\section{PS121 \\ Atypische Präsentation einer nephropathischen Cystinose mit langsam progredienter Niereninsuffizienz und ausgeprägter Osteomalazie bei einem Patienten syrischer Herkunft}

Jan Schiefer'; Juliane Peters'; Hermann-Josef Gröne ${ }^{2}$; Martin Zenker ${ }^{3}$; Carsten Bergmann ${ }^{4}$; Brigitta Witte; Peter Mertens ${ }^{1}$; Christos Chatzikyrkou ${ }^{1}$

'Universitätsklinikum Magdeburg, Magdeburg; ${ }^{2}$ Deutsches Krebsforschungszentrum Heidelberg, Heidelberg; ${ }^{3}$ Medizinische Fakultät der Otto-von-Guericke Universität Magdeburg, Magdeburg; ${ }^{4}$ Universitätsklinikum Freiburg, Freiburg im Breisgau

Fallvorstellung: Die Zuweisung des 36-jährigen syrischen Patienten aus der Orthopädie erfolgte zur Abklärung einer Osteomalazie und Niereninsuffizienz. Wiederholte Knochenbrüche der Beine mit insuffizienter Frakturheilung (Abb. 1) sowie eine Hyperlordose seien seit dem 13. Lebensjahr aufgetreten. Seine Schwester sei mit ähnlicher Symptomatik im Jugendalter verstorben. Außer einer zeitweise substituierten Hypothyreose bei vermuteter Hashimoto-Thyreoiditis sind keine Vorerkrankungen bekannt. Zusätzlich zur Nierenfunktionseinschränkung (eGFR $38 \mathrm{ml} / \mathrm{min}$ ) zeigte sich eine generalisierte Funktionsstörung der proximalen Tubuli im Sinne eines kompletten De-Toni-Fanconi-Syndroms mit Hypokaliämie, Hypophosphatämie, renal tubulärer Azidose, tubulärer Proteinurie, Aminoazidurie und Glukosurie. In der Nierenbiopsie präsentierten sich lichtmikroskopisch eine fokal-segmentale, fokal-globale Glomerulosklerose und ein ausgeprägter chronischer tubulointerstitieller Schaden mit abgeflachten und segmententdifferenzierten Tubuli. Elektronenmikroskopisch (EM) fielen langstreckig fehlende Podozytenfußfortsätze auf. Es erfolgte eine genetische Untersuchung, wo eine pathogene Missense-Mutation C.451A > G (Arg161Gly) im Gen des lysosomalen Cystintransporters (CTNS) homozygot nachgewiesen wurde. Die nachgeforderte Elektronenmikroskopie zeigte in den Zellen der proximalen Tubuli mit hyperdensem Material prall gefüllte Lysosomen, teilweise mit Membranruptur (Abb. 2). Es wurde eine nephropathische Cystinose (juvenile Form) mit Endokrinopathie und sekundärer Osteomalazie im Rahmen der Tubulopathie diagnostiziert. Neben der Elektrolyt-, Bicarbonat- und Vitamin D-Substitution wurde eine Cystin-entspeichernde Therapie mit Mercaptamin-hydrogentartrat eingeleitet. Eine augenärztliche Untersuchung steht noch aus.
Schlussfolgerungen: Der Fall verdeutlicht, dass bei Osteomalazie frühzeitig an eine komplexe metabolische Osteopathie gedacht werden sollte, bei der die Niere oft eine Rolle spielt. Es müssen metabolische, hereditäre, autoimmune und toxische Einflüsse bedacht werden. Bei relativ homogenem mitteleuropäischem Kollektiv ist die Cystinose global eine Erkrankung mit variablem Erscheinungsbild. Bei Europäern wird in $75 \%$ der Fälle eine 57-kb-Deletion im CTNSGen mit Funktionsverlust des Proteins beobachtet, die mit schwerer Symptomatik ab dem Kleinkindalter, Dialyse-pflichtiger Niereninsuffizienz mit ca. 10 Jahren und Enzephalopathie im Erwachsenenalter einhergeht. Am zweithäufigsten ist die adulte, rein okuläre Form. In türkischen und persischen Fallserien werden verschiedene Punktmutationen beschrieben. Bei unserem Patienten findet sich eine intermediäre Symptomatik mit fortgeschrittener, aber keiner terminalen Niereninsuffizienz. Zudem hat er hat ein Studium erfolgreich abgeschlossen und weist keine kognitiven Störungen auf.

\section{PS122 \\ REAL WORLD SAFETY OF VEDOLIZUMAB IN INFLAMMATORY BOWEL DISEASE: A META-ANALYSIS}

Stefan Schreiber'; Axel Dignass; L. Peyrin-Biroulet; M Mosli; Greg Hather; Dirk Demuth; Aimee Blake; R Curtis; Mona Khalid; Edward V. Loftus

\section{'UKSH Campus Kiel, Kiel; Germany}

Introduction: Vedolizumab (VDZ), a gut-selective monoclonal anti-a4 $\beta_{7}$-integrin antibody, is used for treatment of Crohn's disease (CD) and ulcerative colitis (UC). Data from large realworld cohorts can further characterise safety events not fully elucidated in a clinical trial setting, such as the risk of serious infections, as identified with anti-tumour necrosis factor a (TNFa) therapy in the TREAT, GISEA and ENCORE registries.

Aims \& Methods: We conducted a systematic review and meta-analysis of realworld safety outcomes reported for VDZ in UC and CD. MEDLINE-, Cochrane-, and EMBASE-indexed publications and conference abstracts $(n \geq 10)$ from May 1, 2014-January 10, 2017 were searched for studies reporting realworld VDZ safety outcomes. Reports for patients $<18$ years of age or for off-label VDZ use were excluded. A meta-analysis was conducted using the DerSimonian-Laird random effects method to obtain a weighted mean of adverse event (AE) rates.

Results: 218 Published studies were identified, with 33 reporting safety rates on 2857 VDZ-treated patients (CD: 1532; UC: 829; unspecified/ other: 36 ; three studies $[n=460$ ] did not report individual UC/CD data) over a VDZ exposure/follow-up period ranging 0.5-18 months (20 studies). Among included studies, the mean age of patients ranged from 21 to 67 years, with mean disease duration ranging from 7 to 16 years. Most VDZ-treated patients (62-100\%) had prior exposure to $\geq 1$ anti-TNFa therapy and $6-64 \%$ of VDZ-treated patients were receiving concomitant corticosteroids and immunomodulators. The most common non-infectious AEs were acne or acne-like lesions ( $7 \%$; $95 \%$ confidence interval $[\mathrm{Cl}] 5-11 \%)$, fatigue $\left(6 \% ; 95 \% \mathrm{Cl}_{3}-15 \%\right)$ and arthralgia (5\%; $95 \% \mathrm{Cl} 3-10 \%)$. The most common infectious AEs were upper respiratory tract infections $(6 \% ; 95 \% \mathrm{Cl}, 3-11 \%)$ and sinusitis $(4 \% ; 95 \% \mathrm{Cl}$ $<1-19 \%)$. Infusion-related reactions occurred in $2 \%(95 \% \mathrm{Cl}<1-4 \%)$ of patients $(n=811)$, and malignancies were reported in $<1 \%$ of patients $(<1-4 \% ; 2$ studies). Overall, the pooled AE rate reported in VDZ-treated patients was $21 \%$ (95\% Cl 14-32\%); $10 \%$ (95\% Cl 6-16\%) for infections, $8 \%(95 \% \mathrm{Cl} 6-10 \%)$ for serious AEs and $7 \%(95 \% \mathrm{Cl} 3-13 \%)$ for serious infections.

Conclusion: Pooled analysis of AE rates across multiple studies support the favourable, long-term benefit-risk profile of VDZ in real-wor- 
Id clinical practice, with low rates of infusion-related reactions, serious infections and malignancies reported, and no identification of new safety signals. These results are consistent with integrated safety data reported for VDZ in six clinical trials (> 4000 patientyears), despite the selection of complex patients failing previous immunosuppressive or biologic therapies. Limitations of incidental reporting in realworld studies include potential underestimates of $A E$ rates and the reporting of AEs not regularly observed in clinical trials; for example, due to the variability in comedication use and sub-optimal screening of prior infections.

\section{PS123 \\ Beeinflussen genetische Varianten von FGFR4 und Klotho die linksventrikuläre Muskelmasse von Patienten mit chronischer Nierenerkrankung?}

Alexander B. Sellier; Sarah Seiler-Mußler; Insa E. Emrich; Danilo Fliser; Adam M. Zawada; Gunnar H. Heine

Universitätsklinikum des Saarlandes, Homburg

Zielstellung: Erhöhte Plasmaspiegel des Fibroblast Growth Factor 23 (FGF23) sind unabhängige Prädiktoren zukünftiger kardiovaskulärer Ereignisse bei chronisch nierenkranken Patienten. Verschiedene rezente tierexperimentelle Daten suggerieren, dass erhöhte FGF23-Spiegel direkt das Myokard über die Aktivierung des FGF-Rezeptors 4 schädigen, während konkurrierende Studien verminderte Plasmaspiegel des löslichen FGF23-Korezeptors Klotho als direkt kardiotoxisch ansehen. In Querschnittsanalysen unserer prospektiven CARE FOR HOMe Studie konnte kein Einfluss von genetischen Varianten von FGFR4 und Klotho auf die linksventrikuläre Muskelmasse festgestellt werden. Ob das Vorliegen dieser genetischen Varianten einen Einfluss auf Veränderungen der linksventrikulären Muskelmasse in Längsschnittanalysen mit mehrjähriger Nachbeobachtung hat, bleibt indes unklar.

Material \& Methoden: Die CARE FOR HOMe Studie rekrutierte 544 chronisch nierenkranke Patienten in GFR Kategorien G 2 bis $G 4$. Von 519 Teilnehmern wurde DNA isoliert und Single Nucleotid Polymorphismen für FGFR4 (Gly388Arg) und Klotho (Phe352Val) mittels qualitativer Real-Time PCR genotypisiert. Zur Bestimmung des linksventrikulären Massenindex (LVMI) wurden Echokardiographien zum Studieneinschluss $(\mathrm{N}=458)$ und zur Nachuntersuchung nach fünf Jahren $(n=254)$ durchgeführt. Die Untersuchungen folgten den Empfehlungen der American Society of Echocardiography und wurden von einem einzigen Arzt durchgeführt.

Ergebnisse: Patienten mit verschiedenen FGFR4 Genotypen unterschieden sich nicht in der Veränderung des LVMI innerhalb des fünfjährigen Beobachtungszeitraum (Gly/Gly: + 0,1 \pm 17,9 g/m², Gly/Arg: + $1,5 \pm 23,1 \mathrm{~g} / \mathrm{m}^{2}$, Arg/Arg: $\left.-7,7 \pm 24,1 \mathrm{~g} / \mathrm{m}^{2}, \mathrm{p}=0,181\right)$. Patienten mit unterschiedlichem Klotho-Genotyp unterschieden sich ebenso nicht signifikant in den Änderungen des LVMI (Phe/Phe: $-0,2 \pm 19,5 \mathrm{~g} / \mathrm{m}^{2}$, Phe/Val: $+0,2 \pm 25,3 \mathrm{~g} / \mathrm{m}^{2}$, Val $/$ Val: $\left.-0,1 \pm 25,2 \mathrm{~g} / \mathrm{m}^{2}, \mathrm{p}=0,995\right)$.

Schlussfolgerung: Genetische Varianten von FGFR4 und Klotho sind nicht mit unterschiedlichen Veränderungen der linksventrikulären Muskelmasse im Verlauf einer chronischen Nierenerkrankung assoziiert. Diese Ergebnisse widersprechen der Hypothese, dass FGF23 und sKlotho kausal an der Entstehung einer linksventrikulären $\mathrm{Hy}$ pertrophie beteiligt sind. Bevor tierexperimentelle Daten in Form von pharmakologischen Interventionen in die klinische Nephrologie übertragen werden können, sind daher weitere Studien an größeren Patientenkollektiven nötig, um einen kausalen Einfluss von FGFR4 oder sKlotho auf die urämische Kardiomyopathie zu untersuchen.

\section{Internisten und das (zentrale) Nervensystem}

\section{PS124 \\ Lambert-Eaton-Syndrom bei kleinzelligem Bronchialkarzinom auf dem Boden einer idiopathischen Lungenfibrose}

Laurentiu Broscaru; Frank Ingenabel; Claudiu Dobre; Martin Müller; Dietrich Gulba

Katholisches Klinikum Oberhausen, St. Marienhospital, Oberhausen

Ein 69-jähriger türkischer Patient, Raucher, war 2013 mit dem Bild einer ambulant erworbenen Pneumonie und eines spontanen Spannungspneumothorax in unserer Klinik vorstellig. Im CT zeigten sich damals interstitielle Infiltrate in der linken Lunge und Emphysem. 2015 wies ein Kontroll-CT eine progrediente Lungenfibrose mit ubiquitärer interstitieller Pneumonie nach, aber auch einen pulmonalen Rundherd im linken apikalen Unterlappen mit linkshilärer Lymphadenopathie. Der Patient gab Belastungsdyspnoe, Husten und Müdigkeit an, hatte Trommelschlegelfinger und auskultatorisch Knistern, eine $\mathrm{pO}_{2}$ von 70 $\mathrm{mmHg}$ und keine Restriktion oder obstruktive Ventilationsstörung. Laborchemisch fanden sich positive ANA (1:1280). ANCA und AntiCCP-AK waren negativ.

Die Diagnose einer idiopathischen Lungenfibrose wurde gestellt und vor dem eventuellen Therapiebeginn erfolgte die EBUS-gesteuerte Biopsie der mediastinalen Lymphadenopathien, die histologisch keine malignitätsverdächtigen Befunde erwies. 2016 stellte sich unser Patient wieder in unserer Klinik vor, mit persistierender Belastungsdyspnoe, Gesamtkörperschwäche und Gewichtsabnahme von 8 kg. Eine Therapie der Lungenfibrose sei noch nicht eingeleitet worden. Im CT fanden sich jetzt eine deutliche Progredienz der Lungenfibrose sowie eine Größenzunahme des vorbeschriebenen Lungenherds und ein zusätzlicher Lungenherd im linken Oberlappen. Die Belastbarkeit des Patienten im 6-min.-Gehtest war deutlich geringer. $\mathrm{pO}_{2}$ lag bei $58 \mathrm{mmHg}$. Eine leichtgradige Restriktion war nachweisbar. Auffällig waren diesmal sehr hohe CK-Werte (über $7000 \mathrm{UI} / \mathrm{I}$ ), wobei eine Therapie mit Statinen verneint wurde. Im Zusammenhang mit den hohen ANA-Titern und der generalisierten Muskelschwäche und da die Herdbefunde auch mit einem entzündlichen Geschehen vereinbar waren, kam infrage eine autoimmune Muskelerkrankung, wie Polymyositis oder "inclusion body" Myositis, die mit der interstitiellen Lungenerkrankung im Zusammenhang sein konnte. Die Elektromyographie war mit einer myopathischen Läsion vereinbar. Die Anti-Jo1-AK waren negativ und die ersten histologischen Befunde der Muskelbiopsates blieben inkonkludent. Zu diesem Zeitpunkt wurde ein Therapieversuch mit Prednisolon begonnen und es kam nach einigen Tagen zu einer subjektiven Verbesserung der Muskelschwäche und der Dyspnoe. Einige Wochen später zeigten die Ergebnisse der Muskelbiopsie aus dem Referenzzentrum doch eine mittelgradige, floride nekrotisierende Myopathie mit einer ausgeprägten, progredienten neurogenen Muskelatrophie. Mittlerweile habe die Muskelschwäche des Patienten stark zugenommen. Bei dringendem Verdacht auf kleinzelliges Bronchialkarzinom mit paraneoplastischem Lambert-Eaton-Syndrom erfolgte eine CT-gesteuerte Lungenbiopsie, die gemischt-zellige Anteile von einem kleinzelligen Bronchialkarzinom und einem Adenokarzinom nachwies. Eine Chemotherapie mit Carboplatin und Etoposid wurde eingeleitet. 5 Monate später verstarb der Patient infolge einer Pneumonie. 


\section{PS125}

\section{Differential diagnosis of vasculitis on the example of Takayasu arteritis}

Alexandra Elies; Stefan Rosenstengel; Johannes Weßling; Peter Baumgart

Clemenshospital, Münster

Case Report: A 50- year old asian female, presented with headache, double vision and general fatigue. At clinical examination blood pressure was $220 / 110 \mathrm{mmHg}$ and there was left sided palsy of the abducens nerve. CT-scan did not reveal ischemic, hemorrhagic or tumorous lesions. After blood pressure reduction, headache and fatigue improved but neurologic symptomps remained. Cerebral MRI showed chronic microvascular lesions due to hypertension, but no ischemia or sinus vein thrombosis. Lumbar puncture did not reveal bacterial infection or autoimmune disease in liquor. Carotid artery walls were thickened at sonography $(1,01 \mathrm{~mm})$ and MRI divulged a high inflammatory response of the aortic arch and the outgoing supraaortal arteries. Lab results showed elevated BSR and CRP, leucocytosis and anemia. Hepatitis $B$ was newly diagnosed by anti-bodies and PCR. Elevation of CMV-IgA-anti-bodies were suspicious for reactivation of CMV-infection. There were no detectable autoimmune anti-bodies beyond unspecific ANA which were highly elevated.

Discussion: Large vessel disease seemed likely, however international classifications did not allow for adequte discrimination between a giant-cell arteritis or Takayasu-Arteritis (TAK). Further secondary vasculitis due to hepatitis had to be considered, as well as the rare case of a CMV associated vasculitis. Also a behcet vasculitis can, in rare cases mimick large vessel arteritis without antibody labwork. Taking into account the ethnic origin, age and especially the inflammation of the extracranial arteries, we concluded on a TAK. High-dosed Steroids (starting with $80 \mathrm{mg} / \mathrm{d}$ and tapered to $15 \mathrm{mg}$ ) within months were administered according to EULAR recommendations (European League against Rheumatism) and all symptoms disappeared and imaging signs of vasculitis improved.

Conclusion: This case of TAK provides multiple diagnostic aspects of relevance for inflammation of the large extracranial vessels.

\section{PS126 \\ Paradoxe Reaktion auf Pipamperon - Fallbericht eines Patienten mit gravierender kombinierter vokaler und multipler motori- scher Tic-Störung}

Richard Eyermann $n^{1,2}$, Helmut Langhof ${ }^{2}$

'Dr. Eyermann, Kinder- und Jugendmedizin, Kinderkardiologie, Sportmedizin, Allgemeinmedizin, München; ${ }^{2}$ Klinik Schönsicht, Rehabilitation für Kinder und Jugendliche, Anschlussheilbehandlung, Kind-Mutter/Vater-Rehabilitation, Berchtesgaden

Pipamperon (Dipiperon ${ }^{\circledast}$ ) gehört zur Wirkstoffklasse der niederpotenten Neuroleptika u. wird in der Psychiatrie gegen aggressive psychotische Zustände u. Schlafstörungen angewendet. Es hat stark sedierende Wirkung, welche die antipsychotische Effekte in der Intensität übersteigt. Pipamperon wird zur Pharmakotherapie der Tic-Störungen empfohlen.

Fallbericht: Ein 17-jähriger Junge wurde zur Behandlung seiner Tic-Störungen stationär aufgenommen. Er litt an unwillkürlichen, plötzlichen, schnellen, wiederholten, arrhythmischen, stereotypen Bewegungen u. Lautäußerungen. Seit 10/2015 bestanden die Tics, zunächst nur multipel motorisch später aber auch vokal, insgesamt an Häufigkeit zunehmend. Unter Psychotherapie (Hypno-, Gesprächs- therapie) 2015/2016 u. Pharmakotherapie durch Tiaprid 6/2016-6/2017 waren die Tics nicht gebessert $u$. die ambulante Behandlung wurde abgebrochen. Der Jugendliche war im Kontakt freundlich u. zugewandt, er ging ganz offen mit seinen vokalen u. motorischen Tics um. Die Symptomatik nahm bei bei schwierigen Themen deutlich zu, aber auch das konnte er reflektieren. Die Stimmungslage wirkte belastet, von suizidalen Gedanken konnte er sich klar u. glaubhaft distanzieren. Der formale $u$. inhaltliche Gedankengang war geordnet. Kein psychotisches Erleben oder Wahrnehmungsstörungen. Keine Schlafstörungen, keine Antriebsstörung.

Methode: MRT Neurokranium (3 T MRT) u. intrakranieller hirnversorgender Arterien (TOF-Angiographie) unter Dotarem i.v.: unauffälliger, altersentsprechenden intra- $u$. extrakranieller Befund mit Ausschluss Aneurysma oder signifikanter Gefäßstenose. Vor-EEG vor Tiaprid: keine Auffälligkeiten. Im Verlauf EEG unter Tiaprid: artefaktreiche aber altersentsprechend unauffällige Wachableitung incl. Provokationsmethoden ohne Hinweis auf epilepsietypische Potenziale.

Aktueller Befund: HN o.B.. Motorik: keine latenten oder manifesten Paresen. Reflexe: MER normal seitengleich auslösbar, keine Pyramidenbahnzeichen. Koordination: ungestört. Sensibilität: intakt. Psychopathologie: unauffällig. EEG: Normales Beta-EEG.

Die Therapie der Tic-Störung beinhaltet:

- Diagnostik, auch komorbider Störungen

- Psychoedukation, Aufklärung, Beratung, Bewältigungsstrategien, Beobachtung, Begleitung

- Pharmakotherapie D2-Rezeptorantagonisten

(Responderrate 70\%, Tic-Reduktion @60-70\%)

Ergebnis: Nach erfolgloser Tiaprid-Therapie hier Beginn einer Behandlung mit Pipamperon in niedriger Dosierung von $1 \mathrm{mg} / \mathrm{kg} / \mathrm{Kg}$, die aber nach paradoxer Reaktion mit starken Aggressionen leider nach 2 Tagen wieder beendet werden musste. Die unerwünschte NW wurde deutschem Bfarm gemeldet, deren große Datenbank bisher über keinerlei Signale verfügte. Der FDA bislang 25 Fälle von Komplikation Aggression bei insgesamt 594 NW unter Dipiperon gemeldet $=4.2088 \%$ (FDA-Reports jeglicher NW Aggression: $22829=0.1431 \%$ ).

Konklusion: Pipamperon(Dipiperon) -sonst bei aggressiven Zuständen eingesetzt, kann paradoxerweise selbst zu aggressivem Verhalten führen bzw. Aggressionen verstärken.

\section{PS127}

Branched-Chain Amino Acids as potential biomarkers of the somatic health state in Bipolar Disorder

Frederike Fellendorf; Martina Platzer; Susanne Bengesser; Rene Pilz; Nina Dalkner; Robert Queissner; Alexandra Rieger; Carlo Hamm; Harald Mangge; Eva Reininghaus

Medizinische Universität Graz, Graz, Austria

Introduction: Bipolar disorder (BD) is a severe psychiatric disease in which not only current depressive and manic episodes, but also comorbidities pose a major challenge for the healthcare system. BD is accompanied by a higher prevalence of somatic comorbidities such as overweight/obesity as well as insulin resistance, leading to a premature mortality compared to the general population. An important aspect of $B D$ research is the identification of biomarkers pertaining to the current stage and course of $\mathrm{BD}$, as well as the state of somatic 
health. The branched-chain essential amino acids (BCAAs), namely valine, leucine and isoleucine, have been proposed as potential biomarkers of an individual's somatic health state, given their influence on protein synthesis and gluconeogenesis inhibition.

Aim: We aimed to analyze the association between BCAAs and metabolic and clinical parameters in euthymic/subsyndromal depressive individuals suffering from $B D$.

Methods: BCAA levels of 141 euthymic/subsyndromal individuals with $B D$ and 141 matched healthy controls $(\mathrm{HC})$ were analyzed by high-pressure lipid chromatography. Clinical psychiatric, anthropometric and metabolic parameters were collected and correlated with BCAAs.

Results: BD and HC did not differ in valine and isoleucine, whereas leucine was significantly lower in BD. We found significant higher levels of BCAAs in the obese and overweight compared to the normal weight group. Furthermore, correlations were shown between BCAAs and anthropometric data, as well as parameters of glucose metabolism. All BCAAs correlated with lipid metabolism parameters in females with BD. There were no associations between BCAAs and long-term clinical parameters of $\mathrm{BD}$. A negative correlation was found between valine and different depression scales, indicating lower valine in higher depressed patients.

Conclusion: Our results support recent findings in mentally healthy cohorts showing that overweight/obesity is associated with higher BCAA levels compared to normalweight. BCAAs induce the activation of pathways, which regulate cell growth and proliferation, protein synthesis and transcription and however lead to insulin resistance and early beta-cell dysfunction. As overweight is known to be associated with less insulin sensitivity leading to an insulin resistance and diabetes mellitus type 2 and we showed a correlation between BCAA levels and HOMA-IR, our results indicate the utility of BCAAs as biomarkers for the current state of health, including in individuals with BD. As $\mathrm{BD}$ patients have a high risk for classification as overweight/obese, in association with comorbid medical conditions (e.g. cardiovascular diseases, insulin resistance), such markers are urgently required. However, beside correlations between depressive symptoms and valine in male individuals with $\mathrm{BD}$, no illness-specific associations between BCAAs and clinical parameters were found in this euthymic/subsyndromal BD patient group.

\section{PS128}

Das Katecholamin-produzierende Ganglioneurom - eine seltene Differentialdiagnose des Phäochromozytoms

Charlotte Fries; Lena Hübner; Nada Rayes; Wiebke Fenske; Anke Tönjes

Universitätsklinikum Leipzig AöR, Leipzig

Fallbericht: Wir berichten über einen 36-Jährigen Patienten mit rezidivierenden Sinustachykardien und sonographisch auffälligem Inzidentalom der rechten Nebenniere. In der Computertomographie des Abdomens stellte sich die circa $11 \times 11 \mathrm{~cm}$ große Raumforderung mit vereinzelten Verkalkungen und erhöhten Hounsfield-Units dar (>15-30). Laborchemisch zeigten sich eine Erhöhung der Normetanephrine ( $428 \mathrm{pg} / \mathrm{ml}$; Referenzbereich $<127 \mathrm{pg} / \mathrm{ml}$ ) sowie ein erhöhtes 3-Metoxytyramin bei ansonsten unauffälliger Hormondiagnostik. Eine intensive Radiotracerbelegung der rechten Nebenniere in der MIBG-Szintigraphie schien die Verdachtsdiagnose eines Phäochromozytoms zu bestätigen, sodass der Patient nach Aufsättigung mit Phenoxybenzamin zur konventionellen Adrenalektomie in unsere Abteilung für Viszeralchirurgie verlegt wurde. In der histologischen Begutachtung zeigten sich spindelige Tumorzellen neben gruppen- förmig angeordneten Ganglienzellen sowie in der Immunhistochemie eine ausgeprägte Positivität gegenüber S-10o bei Negativität gegenüber Chromogranin. Statt der Verdachtsdiagnose Phäochromozytom konnte demnach ein Ganglioneurom (GN) diagnostiziert werden.

Diskussion: In einem geringen Prozentsatz aller adrenalen Inzidentalome (0.3-2\%) ergibt sich histologisch ein GN. Diese seltenen, in aller Regel gutartigen und asymptomatischen Tumore des sympathischen Nervensystems treten meist im Kindes- und jungen Erwachsenenalter auf und betreffen überwiegend die sympathischen Ganglien des Mediastinums und Retroperitoneums, seltener das Nebennierenmark. Eine Katecholaminproduktion von GN wird in bis zu $30 \%$ der Fälle beobachtet, die Häufigkeitsangaben schwanken jedoch aufgrund der geringen Fallzahlen stark. Besteht eine Katecholaminproduktion, zeigt sich meist auch eine positive MIBG-Anreicherung in der Szintigraphie, sodass sich eine präoperative Differenzierung zum Phäochromozytom aufgrund der unklar definierten bildgebenden Kriterien des GN extrem schwierig gestaltet. Ein GN wird in circa $65 \%$ aller Fälle präoperativ fehldiagnostiziert, eine sichere Diagnose erlaubt weiterhin nur die histologische Begutachtung. Aufgrund der exzellenten Prognose und nur in Einzelfällen berichteten Malignität muss nach Entfernung im Gesunden keine regelhafte adjuvante Therapie oder Nachsorge erfolgen.

Abbildung 1a: Positive Radiotracerbelegung in der MIBG-Szintigraphie

Abbildung 1b: Nebennierenresektat mit teils myxoidem, teils eingebluteten Tumor $(13,1 \times 12,9 \mathrm{~cm})$

\section{PS129}

\section{NUCB2/Nesfatin-1 zeigt bei adipösen Frauen einen positiven} Zusammenhang mit gestörtem Essverhalten

Miriam Goebel-Stengel'; Tobias Hofmann ${ }^{2}$; Elena Weibert ${ }^{2}$; Ulf Elbelt ${ }^{3}$; Matthias Rose ${ }^{3}$; Andreas Stengel ${ }^{4}$

${ }^{1}$ HELIOS Klinikum Zerbst/Anhalt, Zerbst; ${ }^{2}$ Charité-Universitätsmedizin Berlin, Berlin; ${ }^{3}$ Charité Universitätsmedizin Berlin, Berlin; ${ }^{4}$ Universitätsklinikum Tübingen, Tübingen

Hintergrund: Nesfatin-1 ist ein anorexigenes Neuropeptid, das zudem eine Rolle bei der Modulation emotionaler Prozesse zu spielen scheint. In eigenen Voruntersuchungen konnten wir eine negative Korrelation zwischen peripheren NUCB2/Nesfatin-1-Spiegeln und psychometrisch gemessenen Angstwerten bei adipösen Männern, jedoch eine positive Assoziationen bei adipösen Frauen feststellen, was auch in Zusammenschau mit weiteren Studien auf eine geschlechtsspezifische Regulation dieses Peptids hindeutet. Tierexperimentell ist die Rolle von Nesfatin-1 in der Regulation der Nahrungsaufnahme bereits etabliert; in humanen Studien fehlen solche Daten allerdings bisher. Ziel der Studie war es, Zusammenhänge zwischen Plasma-NUCB2/Nesfatin-1 und gestörtem Essverhalten in einer gemischt geschlechtlichen Patientengruppe mit breitem Gewichtsspektrum zu untersuchen.

Methoden: Wir schlossen 243 Patienten (176 weiblich, 67 männlich) ein, die aufgrund von Anorexia nervosa $(n=66)$ oder Adipositas $(n=143)$ und damit assoziierten Komorbiditäten stationär behandelt wurden. Die eingeschlossenen normalgewichtigen Patienten $(n=33)$ wurden aufgrund von somatoformen, depressiven oder Angststörungen behandelt. Parallel zu den Blutentnahmen wurde die essstörungsspezifische Psychopathologie mittels Eating Disorder Inventory-2 (EDI-2, Werte 0-100) erfasst. NUCB2/Nesfatin-1-Plasmaspiegel wurden mit ELISA gemessen. 
Ergebnisse: Die gesamte Studienpopulation zeigte mit einem durchschnittlichen EDI-2-Summenwert von $42,7 \pm 14,6$ (Spanne 5-80) eine essstörungsspezifische Symptomatik. Anorektische $(45,5 \pm 17,3)$ und adipöse Patientinnen $(47,9 \pm 10,5)$ wiesen signifikant höhere EDI-2-Werte als die normalgewichtige weibliche Vergleichsgruppe $(25,6 \pm 10,7, p$

\section{PS130 \\ Adipokines and the unfavorable metabolic profile in bipolar disorder}

Martina Platzer; Frederike Fellendorf; Carlo Hamm; Riccarda Hartleb; Alexander Maget; Rene Pilz; Robert Queissner; Harald Mangge; Sieglinde Zelzer; Eva Reininghaus

Medizinische Universität Graz, Graz, Austria

Introduction: Overweight and obesity are prevalent in patients with bipolar disorder (BD) and are often associated with medical comorbidities like diabetes or the metabolic syndrome which in turn contribute to the heightened mortality in this population and can also worsen the psychiatric prognosis. Adipokines like leptin and adiponectin are crucial to energy homeostasis and may play a role in the metabolic disturbances seen in BD.

Objective: Herein, we sought to examine differences in adiponectin and leptin levels between individuals with BD and healthy controls and the possible association between adipokines and clinical and metabolic parameters.

Methods: We assessed serum adiponectin and leptin levels as well as metabolic, anthropometric and psychometric parameters in 111 individuals with $\mathrm{BD}$ (euthymic or with mild to moderate depression) and 11 matched healthy controls. A detailed psychiatric and medical history was obtained from all participants.

Results: A significant difference in serum adiponectin levels between patients and controls was found. Furthermore, an association between adiponectin and fasting glucose, high density lipoprotein, low density lipoprotein (only in men) and c-reactive protein (only in women) was found in patients but not in healthy controls. Serum adiponectin was lower in male patients taking lithium than in those taking other mood stabilizing medication; however, this difference was only significant in the male subgroup.

Conclusion: Adiponectin has already been identified as a potential independent risk factor for metabolic syndrome in previous investigations; our findings further attest to the unfavorable metabolic profile in patients with BD. It has been previously demonstrated that lithium therapy may lead to reduced adiponectin levels and although due to the cross-sectional design of this investigation an interpretation of the direction of this association cannot be drawn, it seems to confirm a negative association between lithium and adiponectin.

\section{PS131 \\ Ratten mit Aktivitäts-basierter Anorexie zeigen eine Aktivierung CRF-haltiger Neurone in verschiedenen Gehirnkernen}

Sophie Scharner ${ }^{1}$; Miriam Goebel-Stengel ${ }^{2}$; Tiemo Friedrich'; Peter Kobelt"; Matthias Rose ${ }^{1}$; Andreas Stengel ${ }^{3}$

${ }^{1}$ Charité Universitätsmedizin Berlin, Berlin; ${ }^{2} \mathrm{HELIOS} \mathrm{Klinikum} \mathrm{Zerbst/Anhalt,}$ Zerbst; ${ }^{3}$ Universitätsklinikum Tübingen Med. Klinik, Tübingen

Einleitung: Die Aktivitäts-basierte Anorexie ( $A B A$ ) ist das etablierteste Tiermodell für Anorexia nervosa (AN). Dennoch sind zugrundeliegen- de Mechanismen, welche an der Reduktion der Nahrungsaufnahme und des Körpergewichts beteiligt sind, nur unzureichend bekannt.

Ziel: In dieser Studie wurde die potentielle Rolle des Stresshormons Corticotropin-releasing Faktor (CRF) in der Pathogenese von AN anhand des ABA-Modells untersucht. Methodik: Weibliche Sprague-Dawley Ratten wurden in vier Gruppen unterteilt ( $n=6 /$ Gruppe): Aktivitäts-basierte Anorexie (ABA, Nahrung für 1,5 h und 24 h Laufradzugang), restriktive Fütterung (RF, Nahrung für 1,5 h), Aktivität (AC, 24 h Laufrad- und Nahrungszugang) und ad libitum ( $A L, 24 \mathrm{~h}$ Nahrung). Die Tiere entwickelten ABA innerhalb von drei Wochen und wurden danach transkardial perfundiert, die Gehirne für Fos- und CRF-Immunhistochemie weiterverarbeitet. Folgende CRF-haltige Gehirnkerne wurden untersucht: paraventrikulärer Nukleus (PVN), dorsomedialer Hypothalamus (DMH), Edinger-Westphal Nukleus (EW), dorsaler Raphe Nukleus (DR), Lokus coeruleus (LC) und Nukleus traktus solitarius (NTS). Ergebnis: $A B A$ führte zu einer erhöhten Anzahl Fos positiver Zellen im PVN (Zellen/Schnitt, MW \pm SEM, ABA: $13,3 \pm 4,5, \mathrm{RF}: 4,8 \pm 2,0$, AC: $3,4 \pm 1,4, A L: 3,1 \pm 1,5)$ im Vergleich zu AL und $A C(p<0,05)$, auch die Zahl Fos/Nesfatin-1 immunreaktiver Zellen war bei ABA $(5,1 \pm 1,3)$ verglichen zu $A L(0,5 \pm 0,3)$ und $A C(0,4 \pm 0,4)$ erhöht $(p<0,01)$. Im DMH steigerte $A B A(29,6 \pm 8,4)$ ebenfalls die Anzahl Fos positiver Neurone im Vergleich zu AL $(2,9 \pm 1,4, p<0,01)$ und $A C(4,9 \pm 3,1, p<0,05)$, nicht aber zu RF $(17,7 \pm 4,4, p>0,05)$. Auch die Zahl CRF positiver Neurone war bei ABA $(27,6 \pm 4,8)$ im Vergleich zu AL $(2,1 \pm 1,8)$ und AC $(4,8 \pm 2,8)$ erhöht ( $p<0,01)$, ebenso die Anzahl Fos/Nesfatin-1 immunreaktiver Zellen bei $A B A(6,5 \pm 1,2)$ verglichen zu $A L(0,5 \pm 0,3, p<0,01)$ und $A C$ $(0,8 \pm 0,7, p<0,05)$. IM EW wurde eine Erhöhung der Anzahl Fos positiver Neurone bei ABA $(7,4 \pm 2,4)$ im Vergleich zu AC $(1,8 \pm 0,7, p<0,05)$ beobachtet, wohingegen die Anzahl Fos/Nesfatin-1 immunreaktiver Zellen nicht signifikant verschieden war (ABA: $2,8 \pm 1,1, \mathrm{RF}: 1,6 \pm 0,6, \mathrm{AC}$ : $0,3 \pm 0,1, A L: 1,2 \pm 0,7, p>0,05)$. Keine Unterschiede zeigten sich im DR, LC und NTS (Daten nicht gezeigt).

Schlussfolgerung: CRF könnte eine Rolle in der Reduktion der Nahrungsaufnahme und des Körpergewichts bei ABA spielen, sowie in emotionale Prozesse unter diesen Bedingungen involviert sein.

\section{PS132}

Aducanumab titration dosing regimen: 24-month analysis from prime, a randomized, double-blind, placebo-controlled Phase 1B study in patients with prodromal or mild Alzheimer's disease

Philipp von Rosenstiel'; Sarah Gheuens'; Tianle Chen'; John O'Gorman'; Ping Chiao'; Grace Wang'; Christian von Hehn'; LeAnnne Skordos'; Christoph Hock $^{2}$; Roger M Nitsch ${ }^{2}$; Samantha Budd Haeberlein ${ }^{1}$; Alfred Sandrock ${ }^{1}$

'Biogen, Cambridge, MA, United States; ${ }^{2}$ Neurimmune and University of Zürich, Zürich, Switzerland; ${ }^{2}$ Neurimmune and University of Zürich, Zürich, Switzerland

Background: Aducanumab (BIIB037), an anti-amyloid beta (A $\beta$ ) monoclonal antibody, is being investigated as a disease-modifying treatment for early Alzheimer's disease (AD). PRIME is an ongoing Phase $1 \mathrm{~b}$ study evaluating the safety, tolerability, pharmacokinetics, and pharmacodynamics of aducanumab in patients (aged 50-90, positive $A \beta P E T$ scan) with prodromal/mild AD. Amyloid related imaging abnormalities-vasogenic edema (ARIA-E) were the main safety finding in an interim analysis of PRIME; these were dose-dependent and more frequent in apolipoprotein $\mathrm{E}_{4}(\mathrm{ApoE} \varepsilon 4)$ carriers.1 $\mathrm{A}$ titration regimen was tested in ApoE $\varepsilon 4$ carriers to explore impact of titration on ARIA incidence. We report 24-month data for both fixed-dose and titration cohorts, including 12 months from the double-blind, placebo-controlled period and 12 months from the long term extension (LTE). 
Methods: During the double-blind phase, patients received aducanumab $(1,3,6$ or $10 \mathrm{mg} / \mathrm{kg}$ fixed or titrated to $10 \mathrm{mg} / \mathrm{kg})$ or placebo q4W for 52 weeks. In the LTE, all patients were assigned aducanumab 3,6 , or $10 \mathrm{mg} / \mathrm{kg}$, fixed or titrated. Except safety, all LTE endpoints, including measurement of $A \beta$ using PET and changes in clinical endpoints, were exploratory.

Results: Of 196 patients initially randomized and dosed in PRIME, 143 were dosed in the LTE and 115 completed treatment at Month 24. Patients from the titration cohort who continued aducanumab up to 24 months showed a reduction in brain amyloid plaque levels, consistent with results observed in fixed-dose cohorts. In placebo-treated patients who switched to aducanumab in the LTE, decreases in amyloid plaque were also observed.

Clinical Dementia Rating-Sum of Boxes (CDR-SB) and Mini-Mental State Examination (MMSE) data suggest a clinical benefit in patients continuing aducanumab over 24 months. Of the 185 patients dosed with aducanumab in PRIME, 46 patients experienced ARIA-E. There were no new cases of ARIA-E in patients who continued the same dose of aducanumab. 4 patients experienced more than one episode of ARIA-E over 24 months of treatment, with 2 additional patients experiencing recurrent ARIA-E after the first year of the LTE. Recurrent events were consistent with other ARIA reported to date; they were typically asymptomatic, and most patients continued the study. Incidence of ARIA-E after switching from placebo to aducanumab was consistent with that reported in the placebo-controlled period.

Conclusions: In patients from titration and fixed-dose cohorts who completed the first year of the LTE, amyloid plaque levels continued to decrease in a dose- and time-dependent manner. Analyses of clinical endpoints suggest continued benefit during the second year of treatment. Recurrent ARIA events were consistent with other ARIA events reported to date. No new safety signals were identified at 24 months. These data support further investigation of aducanumab in in the ENGAGE/EMERGE Phase 3 trials.

1. Sevigny J et al. Nature. 2016;537:50-56.

Interdisziplinarität in der Inneren Medizin III \& Multimorbidität und Polypharmazie

\section{PS134}

\section{Does the Proton Pump Inhibitor Use in Kidney Transplanted} Patients Affect Renal Function?

Dominik Flothow; Barbara Suwelack; Rene Schmidt; Hermann Pavenstädt; Katharina Schütte-Nütgen; Stefan Reuter

University Hospital Münster, Münster

Background: Recently, proton pump inhibitor (PPI) intake has been linked to acute kidney injury and chronic kidney disease. In the setting of renal transplantation, it was hypothesized that PPI have a clinically relevant drug interaction with mycophenolate mofetil, potentially leading to rejection. However, current data is inconsistent and the causal pathways are still unknown. Objective of this study was to assess the effect of PPIs on renal function and rejection rate in kidney transplanted patients.

Methods: We performed a single center, explorative, retrospective study of 460 patients who received a kidney transplant between May 2010 and July 2015. All included patients received PPI therapy at primary discharge after transplantation. Median follow-up time was 3.3 years. PPI prescription was assessed in half-year intervals. Primary outcome parameters were eGFR, change in eGFR ( $\triangle \mathrm{eGFR}$ ), 30\% and $50 \%$
eGFR decrease for different time periods (half year to five years). Secondary outcome parameters were: delayed graft function (DGF) and BPAR in the second half of the first year and second year. Two patient groups were formed, in regard of PPI-intake $(n=369)$ and non-intake $(n=84)$ half a year after transplantation. These were compared for eGFR and $\triangle$ eGFR outcomes.

Results: $30 \%$ and 50\% eGFR-decrease showed no association with PPI mean daily intake in our patient cohort (multivariable analysis). In the group comparison, the PPI-intake group showed a significantly higher mean GFR $(p<0.05)$, this however was unlikely a result of PPI-therapy, but rather group selection. No difference in the groups was found for $\triangle \mathrm{eGFR}$ (over up to 3.5 years). In the entire cohort DGF was recorded in $84(18.3 \%)$ patients and correlated with baseline PPI-dose (multivariable analysis). BPAR occurred in 32 patients in months 6-12, and in 39 in the second year after transplantation but did not relate to mean PPI daily intake. In the analysis of the different BPAR types, only antibody mediated rejections in the second year showed correlation with increased PPI-intake $(p<0.05)$.

Conclusion: Our findings indicate that prolonged PPI-intake has no relevant adverse effect on transplant function or outcome. They also highlight the importance of examining changes in eGFR ( $\triangle \mathrm{eGFR}$ ) rather than single eGFR measurements in similar studies, to avoid confounding. Polypharmacotherapy however, remains a problem in renal transplant recipients, and it is thus advisable to question necessity of PPI-prescriptions when clear indications are missing. 


\section{PS135}

\section{Rivaroxaban + Grapefruit $=$ Blutung}

Erik Rafflenbeul'; Antje Walter ${ }^{2}$

${ }^{1}$ Asklepios Klinik Barmbek, Hamburg; ${ }^{2}$ Asklepios Klinik Altona, Hamburg

Einleitung und Fallbeschreibung: Eine 74jährige Patientin stellte sich in der ZNA aufgrund einer plötzlichen Epistaxis, sowie multiplen Hämatomen der Haut vor. Zwei Wochen vor der aktuellen Vorstellung erfolgte eine Verordnung von Rivaroxaban (2omg 1x/d) aufgrund von paroxysmalem Vorhofflimmern. Nebenbefundlich Angabe eines Konsum einer Grapefruit jeden Morgen.

Vorerkrankungen: Paroxysmales Vorhofflimmern - $\mathrm{CHA}_{2} \mathrm{DS}_{2}-\mathrm{VASC}=$ $4(\mathrm{H}+\mathrm{D}+\mathrm{A}+\mathrm{Sc})$. Arterieller Hypertonus. Diabetes mellitus Typ II (diätetisch geführt)

Medikation: Rivaroxaban 20mg 1-0-0. Metoprololsuccinat 47,5mg 1-0-1. Ramipril 2,5mg 1-0-0

Körperlicher Untersuchungsbefund bei Aufnahme: Ekchymose beider Unterschenkel, sowie Unterarme und Bauchhaut. Sonst o.p.B.

Laborbefunde bei Aufnahme: INR > 7, PTT 180 Sekunden (Normwert: 22-34 Sekunden) Rivaroxaban-level $173 \mathrm{ng} / \mathrm{ml}$ (keine Referenzwerte vorhanden), Haemoglibin $10.6 \mathrm{~g} / \mathrm{dl}$ (Normwert: 12-16 g/dl). Normale GPT, GOT, yGT, AP und Lipase. Kreatinin 0,9 mg/dl (Normwerte $0,8-1,1 \mathrm{mg} / \mathrm{dl}$ )

Weiterer klinischer Verlauf: Rivaroxaban wurde unmittelbar pausiert. Zwei Tage nach Aufnahme auf die IMC-Unit war der INR weiter $>7$ und die PTT mit 91 Sekunden deutlich verlängert. Hämoglobin fiel auf 5,1 g/dl. Ein durchgeführtes CT des Abdomens bei Angabe von Flankenschmerzen zeigte multiple retroperitoneale Hämatome. 2000 i.E. Prothrombinkonzentrat (PPSB) und zwei Erythrozytenkonzentrate wurden verabreicht. Koloskopie und Ösophagogastroduodenoskopie zeigten keine Blutungsquellen. Eine Thrombozytenaggregationsfunktionstestung war ohne pathologischen Befund. Kein Anhalt für eine Von-Willebrand-Syndrom. Mit einem HAS-BLED-Score von $3(\mathrm{~B}+\mathrm{L}+\mathrm{E})$ und einem $\mathrm{CHA}_{2} \mathrm{DS}_{2}$-VASc von 4 wurde die Indikation zu einem interventionellen Vofhofohrverschluss gestellt. Der Patientin wurde komplikationslos ein 20mm AMPLATZER Cardiac Plug (St. Jude Medical) implantiert.

Schlussfolgerung: Im klinischen Alltagen werden Arzneimittelinterakionen zunehmend bedeutsamer. Suffiziente Daten zu genauen Effekten von Interaktionen fehlen. In dem gezeigten Patientenfall ist die Interaktion zwischen Rivaroxaban und dem Grapefruitkonsum offensichtlich. Diverse Studien zeigen, dass Grapefruit ein potenter Inhibitor von $\mathrm{CYP}_{3} \mathrm{~A}_{4}$ ist und mit diversen Medikamenten in Wechselwirkung tritt. Rivaroxaban ist ein Substrat von $\mathrm{CYP}_{3} \mathrm{~A}_{4}$.. Auf PubMed sind keine Ergebnisse zu "Rivaroxaban" oder "Xarelto" UND "grapefruit" oder "grapefruitsaft" bzw. "grapefruitjuice" zu finden. In der Fachinformation zu Rivaroxaban (Stand 10/2017) finden sich keine Bemerkungen. In einer amerikanischen Kommentierung der FDA findet sich jedoch folgende Stellungnahme: "Grapefruit juice may also increase plasmaconcentrations of XARELTO ${ }^{\circledR}$ and should be avoided." Die Konsequenz hätte sein können die Rivaroxabantherapie oder den Konsum von Grapefruits zu beenden. Bei jedoch eröhtem HASBLED-Score und der Indikaion zur Antikoagulation bei hohem Thrombembolierisiko wurde die Entscheidung zum Vorhoffohrverschluss gestellt und dies wird durch die aktuellen Leitlinien unterstützt.
PS136

Lebensqualität und Schmerzen vor und nach Behandlung mit einem neuen transdermalen Fentanylpflaster - erste Ergebnisse der Relief-Study

Roxane Schulten'; Tanja Kottmann ${ }^{2}$; Christoph Dietrich ${ }^{3}$

${ }^{1}$ Dr. Ausbüttel Pharma GmbH, Witten; ${ }^{2} \mathrm{CRO}$ Dr. med. Kottmann GmbH \& Co. KG, Hamm; ${ }^{3}$ Bethlehem-Gesundheitszentrum Stolberg GmbH, Stolberg

Hintergrund: Tumoren führen oft zu schwersten Schmerzen, die weltweit in der Hälfte der Fälle nicht adäquat behandelt werden. Weiterhin verursachen chronische nicht-maligne Erkrankungen häufig schwerste Schmerzzuständen, die ebenfalls oftmals nur unzureichend behandelt werden. Beide Ursachen bedingen eine deutliche Reduzierung der Lebensqualität. Durch ein neues transdermales Fentanylpflaster sollen die Schmerzen effektiv behandelt und hierdurch die Lebensqualität verbessert werden. Die neue transdermale Fentanylmatrix (TFM) ist mit Aloe Vera beschichtet, wodurch Hautreizungen vermieden und Unverträglichkeiten minimiert werden sollen.

Methoden: Für die prospektive, einarmige multizentrische Studie wurden zwischen 12/16 - 07/17 112 Patienten rekrutiert, die mit dem neuen TFM mit den Wirkstärken zwischen $12,5-100 \mu \mathrm{g} / \mathrm{h}$ therapiert wurden. Die Patienten wurden vor Beginn der Therapie und nach 6 Wochen untersucht, wobei die Schmerzstärke, die Nebenwirkungen und die Lebensqualität (SF-12) erhoben wurden.

Ergebnisse: Für die Studie wurden bisher 112 Patienten (71 \pm 13 Jahre, $67,9 \%$ männlich) rekrutiert. Ursächlich für die Schmerzbehandlung war am häufigsten Arthrose / Osteoporose $(60,4 \%)$, gefolgt von malignen Erkrankungen (12,3\%) und rheumatoider Arthritis (9,4\%), während restliche Erkrankungen $17,9 \%$ betrugen. Vor Therapiebeginn wurden $62,5 \%$ der Patienten mit antipyretischen antiphlogistischen Analgetika (z.B. ASS), 53,6\% mit nicht-sauren antipyretischen Analgetika (z.B. Metamizol) 35,7\% mit einem Analgetikum der WHO-Klasse II (z.B. Tramadol) und 48,2\% mit einem anderen Fentanyl- bzw. Morphinderivat behandelt. In 50,4\% aller Fälle wurde die bisherige Schmerzmedikation als nicht genügend angesehen:

Die Haftung des Pflasters wurde in $94,6 \%$ und die Schmerzreduktion in $84,7 \%$ als sehr gut oder gut bewertet. Die systemische Verträglichkeit war in $94,5 \%$ und die Hautverträglichkeit in $94,6 \%$ sehr gut oder gut. Innerhalb von 6 Wochen traten in 5,6\% leichte und in 0,9\% starke Hautreaktionen auf. Die körperliche Summenskala des SF-12 verbesserte sich unter der Therapie mit der TFM signifikant von $27,5 \pm 1,1$ auf $36,7 \pm 8,8$ Punkte $(p<0,001)$ und die psychische Summenskala von $35,1 \pm 11,1$ auf $45,5 \pm 8,9$ Punkte $(p<0,001)$.

Schlussfolgerungen: Im hausärztlichen Alltag war die Indikation zur Behandlung mit einem transdermalen Fentanylpflaster in mehr als $2 / 3$ der Fälle eine chronisch-degenerative Erkrankung. In dieser Klientel ließ sich innerhalb von 6 Wochen mit der neuen TFM eine sehr gute oder gute Schmerzreduktion erzielen. Zusätzlich kann durch das neue TFM eine hochsignifikante Verbesserung der Lebensqualität erreicht werden. Die systemische Verträglichkeit, die Hautverträglichkeit und die Haftung der neuen TFM mit der Aloe Vera-Beschichtung werden ganz überwiegend als sehr gut oder gut beurteilt. 


\section{PS137}

\section{Genetische Untersuchung bei Statinmyopathie im klinischen Alltag}

Steffi Szymczak'; Brigitte Öhm²; Christian Dorbath²; Peter Grützmacher ${ }^{2}$

${ }^{1}$ AGAPLESION MARKUS Krankenhaus, Frankfurt; ${ }^{2}$ AGAPLESION Markus-Krankenhaus, Frankfurt

Bei der Therapie mit Statinen werden als häufigste Nebenwirkung muskuläre Beschwerden beobachtet. Der Schweregrad und klinische Symptomatik sind sehr variabel. Diagnosestellung und Auswahl eines verträglichen Statins sind klinisch aufwendig. Mittlerweile wurden verschiedene genetische Polymorphismen im Statinmetabolismus identifiziert, welche das Risiko einer Statinmyopathie erhöhen können und mit Hilfe eines neuen Tests untersucht werden können [3-Hydroxy-3-Methylglutaryl-Coenzym-A-Reduktase (HMG-Gen), Coenzym Q2 (COQ2-Gen), leberspezifischer Anionentransporter (SCLO1B1-Gen), ATP-Bindungskasetten (ABCG2-, $A B C B 1-G e n)] . V a r i-$ anten der Transporterproteine SCLO1B1-, $A B C G_{2}-, A B C B 1$ führen zur verminderten Aufnahme der Statine in die Leberzelle, was zur Unterdosierung verschiedener Statine und extrahepatischer Überdosierungen mit Toxizität führen kann. Veränderungen des HMG-Gens bedingen eine verminderte Wirksamkeit der Statine. Coenzym Q2 führt bei Variationen bei allen Statinen zu Myopathien.

Patienten/Methode: Wir haben in einer Fallserie von 28 Patienten, die in unserer Lipidsprechstunde mit V.a. Statinmyopathie vorgestellt wurden, diesen Test durchgeführt.

Ergebnisse: Insgesamt zeigten 19/28 (68\%) der Patienten Auffälligkeiten, in 9/29 (32\%) der Fälle war der Test unauffällig. Fast die Hälfte der Pat. (13 von $28=46 \%$ ) wiesen Auffälligkeiten im COQ2-Gen auf. Davon waren 12/13 mit weiteren genetischen Veränderungen der Transporterproteine assoziiert. Insgesamt zeigten 18/28 (64\%) eine oder mehr Genvarianten der Transporterproteine (SCLO1B1: 8/28 (29\%); ABCG2: 7/28 (25\%); ABCB1: 8/28 (29\%). Auffälligkeiten im HMGCR-Gen wurden bei keinem Patienten festgestellt.

Schlussfolgerung: Der Test kann helfen, die klinische Verdachtsdiagnose zu sichern und zur Bestätigung der Diagnose "Statinmyopathie" dienen. Er eignet sich zur Auswahl und Dosisfindung des individuell verträglichsten Statins und kann zur Steigerung der Compliance führen. Der klinische Nutzen pharmakogenetischer Tests zur Statinmyopathie sollte unbedingt weiter evaluiert werden.

\section{PS139 \\ Nur wieder ein Alkoholiker? Der lange Weg zur Diagnose einer Sarkoidose.}

Kathrin Untersteller; Moritz Bewarder; Claudia Simone Lennartz; Danilo Fliser; Gunnar Henrik Heine

Universitätsklinikum des Saarlandes, Homburg, Homburg;

Fallvorstellung: Die stationäre Vorstellung des 51-jährigen Patienten in der Nephrologie erfolgte im Oktober 2017 bei akuter Nierenschädigung (Kreatinin bei Aufnahme 4,2 mg/dl, Harnstoff 122 mg/dl, Vorwerte im Normbereich), a. e. prärenal bei iatrogener Hypotonie unter seit einigen Wochen gesteigerter Diuretikaeinnahme. Die klinische Untersuchung zeigte bis auf ein blasses Hautkolorit bei laborchemischer Anämie von $10 \mathrm{~g} / \mathrm{dl}$ keine Auffälligkeiten. Laut Vordiagnosen war ebenfalls eine ethyltoxische Leberzirrhose bekannt, ED 06/2017.
Bei Aufnahme betrug der Child-Pugh-Score 6 Punkte, entsprechend einem Stadium A der Leberzirrhose. Anamnestisch gab der Patient einen Alkoholkonsum von circa $300-500 \mathrm{ml}$ Rotwein pro Tag an, welchen er bei Diagnose der Leberzirrhose umgehend eingestellt hatte (bei Aufnahme Transaminasen normwertig, gamma-GT $130 \mathrm{U} / \mathrm{I}$ ).

Klinisch beklagte der Patient im Verlauf des Aufenthaltes rezidivierende Athralgien vor allem der Handgelenke, eine eingeschränkte körperliche Belastbarkeit sowie ständige Müdigkeit. Innerhalb der letzten Monate habe er 30 kg abgenommen, zunächst gewollt, im Verlauf dann aufgrund von Appetitmangel.

Methode: Zur Abklärung von osteolytischen Läsionen, welche in einem auswärtigen Low-Dose-CT festgestellt wurden, erfolgte ein Staging-CT des Hals, Thorax und Abdomens. Bei den Osteolysen handelte es sich um Hämangiome und eine feinfleckige Demineralisierung. Weiterhin zeigte sich eine mediastinale und bihiläre Lymphadenopathie, passend zu einer Sarkoidose. Laborchemisch waren ACE mit $92 \mathrm{U} / \mathrm{I}$ (Norm 20-70 U/I) und der IL2-Rezeptor mit $2882 \mathrm{U} / \mathrm{ml}$ (Norm 158-623) erhöht. Zur weiteren Diagnostik wurde eine Bronchoalveoläre Lavage durchgeführt, welche eine milde lymphozytäre Alveolitis mit sarkoidosetypischen $\mathrm{CD}_{4} / 8$ Quotient von 7,5 zeigte. In der anschließend transjugulär durchgeführten Leberbiopsie zeigte sich eine chronische aktive, partiell granulomatöse Hepatitis mit portaler und periportaler Fibrose sowie inkompletter Leberzirrhose, welche mit der klinischen Verdachtsdiagnose einer Sarkoidose vereinbar waren. Unter Pausierung der antihypertensiven Medikation besserte sich die Nierenfunktion sukzessive, das Kreatinin lag zuletzt bei 1,48 mg/ dl. Weiterhin wurde eine Steroidtherapie mit Prednison $50 \mathrm{mg}$ täglich begonnen. Hierunter kam es bereits nach wenigen Tagen zu einer guten Beschwerderegredienz.

Fazit: Auch bei Alkoholanamnese ist es sinnvoll, eine Umfelddiagnostik zu betreiben und den Patienten nicht gleich als "Alkoholiker" zu titulieren. 


\section{Prävention und Rehabilitation}

\section{PS140}

Diabetes mellitus increases the risk of thromboembolic complications and major bleedings in patients receiving oral anticoagulation therapy - Results from the thrombEVAL study

Christoph Bickel'; Mario Klaas ${ }^{2}$; Lisa Eggebrecht ${ }^{3}$; Sebastian Göbel ${ }^{4}$; Heidrun Lamparter ${ }^{2}$; Markus Nagler ${ }^{3}$; Karsten Keller ${ }^{3}$; Volker Schmitt ${ }^{4}$; Michael Lauterbach $^{5}$; Christine Espinola-Klein ${ }^{6}$; Roland Hardt ${ }^{7}$; Thomas Münzel ${ }^{8}$; Philipp S. Wild ${ }^{4}$; Jürgen Prochaska ${ }^{4}$

${ }^{1}$ Federal Armed Forces Central Hospital Koblenz, Koblenz; ${ }^{2}$ University Medical Center of the Johannes Gutenberg-University Mainz," Mainz; ${ }^{3}$ University Medical Center of the Johannes Gutenberg University Mainz, Mainz; ${ }^{4}$ University Medical Center of the Johannes Gutenberg-University Mainz, German Center for Cardiovascular Research (DZHK), Partner Site Rhine Main, Mainz; ${ }^{5}$ Barmherzige Brüder Hospital Trier, Trier; ${ }^{6}$ University Medical Center of the Johannes Gutenberg-University Mainz, Mainz; ${ }^{7}$ University Medical Center Mainz, Johannes Gutenberg University-Mainz, Mainz; ${ }^{8}$ University Medical Center of the Johannes Gutenberg University Mainz, German Center for Cardiovascular Research (DZHK), Partner Site Rhine Main, Mainz, Mainz

Background: Diabetes mellitus (DM) represents a known cardiovascular risk factor, but the information regarding the influence of DM on the quality of oral anticoagulation under vitamin $\mathrm{K}$ antagonist therapy $(\mathrm{OAC})$ in the context of prevention of thromboembolic events is limited.

Methods: Information on 2,011 individuals under OAC was available for analysis from the prospective multi-centre thrombEVAL study (NCT01809015). Data were obtained from clinical visits, computer-assisted interviews, and laboratory measurements. Time in therapeutic range (TTR) was calculated as a surrogate of quality of OAC therapy. During a three year follow-up period, information on study endpoints (e.g. bleeding, thromboembolic events, hospitalization, and all-cause mortality) was assessed, validated by medical records and subsequently adjudicated.

Results: Overall, $30.1 \%$ of the study participants were diabetics ( $\mathrm{n}=$ 598). $13.2 \%$ of diabetics were treated with a diabetes diet, $38.8 \%$ with oral antidiabetics. $48 \%$ of diabetics were insulin dependent (IDDM). In diabetics, TTR was significantly reduced at $66.53 \%$ compared to a TTR of 70.42 in nondiabetics $(p=0.010)$. The event with regard to hospitalization under OAC therapy was significantly increased in diabetics with 75.99/100 patient-years (py) vs. 57.39/100-py ( $p$
PS141

Validierung der Kurzform der Allgemeinen Selbstwirksamkeitsskala (GSE-6) in der internistischen Rehabilitation

\section{Martin Brünger; Karla Spyra}

Charité - Universitätsmedizin Berlin, corporate member of Freie Universität Berlin, Humboldt-Universität zu Berlin, and Berlin Institute of Health, Berlin

Hintergrund: Selbstwirksamkeitserwartung (Bandura, 1997) spielt In gesundheitspsychologischen Modellen eine zentrale Rolle zur Erklärung von Gesundheitsverhalten wie körperliche Aktivität und Ernährung (Schwarzer et al., 2011) und findet daher therapeutische Berücksichtigung beispielsweise in der Rehabilitation. Zur Erfassung des Konstrukts am weitesten verbreitet ist die allgemeine Selbstwirksamkeitserwartungsskala (GSE) (Schwarzer, Jerusalem, 1995). Allerdings ist es aufgrund des Umfangs nicht immer möglich, die GSE einzusetzen. Daher wurde die Kurzform GSE-6 vorgeschlagen (Romppel et al., 2013). Ziel dieser Arbeit ist die Bestimmung psychometrischer Eigenschaften und der Validität der GSE- 6 bei Patienten in der internistischen Rehabilitation.

Methodik: Grundlage dieser Analyse ist eine Beobachtungsstudie mit Versicherten der Deutschen Rentenversicherung Bund (Brünger et al., 2016). Für $n=1.350$ Personen mit Bewilligung einer internistischen Rehabilitation (Kardiologie, Pulmologie, Gastroenterologie \& Onkologie) liegen Angaben aus einer postalischen Befragung zur allgemeinen Selbstwirksamkeitserwartung (GSE), psychischen Beeinträchtigung (PHQ-4), Depressivität/Angst (HADS), Beeinträchtigung durch Schmerzen (PDI), Gesundheitsverhalten (IRES), sozialen Unterstützung (IRES) und subjektiven Arbeitsfähigkeit (WAS) vor. Es werden psychometrischen Eigenschaften, die konkurrente Validität mit Korrelationen nach Spearman zu den genannten Beeinträchtigungen und Ressourcen und die faktorielle Validität mithilfe konfirmatorischer Faktorenanalysen (CFA) im Vergleich beider Versionen (GSE-6/GSE) geprüft.

Ergebnisse: Die Mittelwerte der Summenwerte von Kurz- und Originalfassung waren vergleichbar: GSE-6 28,8 $(5,7)$ Punkte; GSE 28,6 $(5,7)$ Punkte. Bei $93,8 \%$ der Rehabilitanden divergierten die beiden Summenwerte um maximal \pm 2 Punkte. Bodeneffekte existierten bei GSE- 6 für 3,0\% der Patienten (GSE: 2,6\%), Deckeneffekte bei o,2\% (GSE: 0,2\%). Die mittlere Trennschärfe der Items lag bei $r=0,68$ für GSE- 6 und bei $r=0,73$ für GSE. Die interne Konsistenz nach Cronbachs alpha betrug 0,88 für GSE- 6 und 0,93 für GSE. GSE- 6 und GSE waren mit $r=0,98$ untereinander korreliert. Die Zusammenhänge zu anderen Beeinträchtigungen und Ressourcen waren jeweils nahezu identisch mit Abweichungen in der Höhe des Korrelationskoeffizienten von maximal 0,02. Multigruppen-CFA bestätigten die eindimensionale Struktur unabhängig von Geschlecht, Alter und Indikationsgruppe.

Diskussion: Es zeigten sich nur geringfügige Abweichungen bei Nutzung der GSE-6 im Vergleich zur Originalversion. Die psychometrischen Eigenschaften liegen für beide Versionen auf einem hohen Niveau. Damit scheint eine Anwendung der Kurzversion in der internistischen Rehabilitation und auch in anderen Reha-Bereichen (Brünger, Spyra, 2017) indikationsübergreifend ohne nennenswerte Abstriche im Vergleich zur Originalversion möglich. Diese Erkenntnis kann zukünftig die Erfassung von Selbstwirksamkeitserwartung vereinfachen. 


\section{PS142}

Ergebnisse aus einer gepolten Analyse der Sicherheitsdaten eines adjuvantierten Herpes Zoster Untereinheit Impstoffkandidaten bestehend aus mehr als $\mathbf{1 4 . 5 0 0}$ Studienteilnehmern ab 50 Jahren

Marta Lopez-Fauqued'; Laura Campora'; Frederique Delannois'; Mohamed El Idrissi'; Edouard Ledent'; Javier Diez-Domingo ${ }^{2}$; Janet McElhaney ${ }^{3}$; Shelly McNeil ${ }^{4}$; Ferdinandus De Looze ${ }^{5}$; Wilfried $\mathrm{Yeo}^{6}$; Fernanda Tamares da Silva'; Johannes Hain $^{7}$

${ }^{1}$ GlaxoSmithKline, Wavre, Belgium; ${ }^{2}$ Fundación para el Fomentode la Investigación Sanitaria y Biomédica (FISABIO), Valencia, Spain; ${ }^{3}$ Health Sciences North Research Institute, Sudbury, ON, Canada; ${ }^{4}$ IWK Health Centre and Nova Scotia Health Authority, Halifax, NS, Canada; ${ }^{5}$ University of Queensland, Brisbane; ${ }^{6}$ University of Wollongong, Wollongong, NSW, Australia; ${ }^{7}$ GlaxoSmithKline GmbH \& Co. KG, München

Hintergrund: Der rekombinante Herpes zoster (HZ) -Untereinheitenimpfstoff $(\mathrm{HZ} / \mathrm{su})$ hat in zwei zulassungsrelevanten klinischen Phase-III-Studien (NCT01165177, NCTo1165229) Wirksamkeit gegen HZ bei Erwachsenen $\geq 50$ und $\geq 70$ Jahren $(\mathrm{J})$ aufgezeigt. Eine gepoolte $\mathrm{Si}-$ cherheitsanalyse der Daten aus diesen beiden Wirksamkeitsstudien wurde durchgeführt, einschließlich einer vergleichenden Analyse von $\mathrm{HZ}$ /su vs. Placebo, um ein umfassendes Verständnis des HZ/su-Sicherheitsprofils zu vermitteln.

Methoden: Zwei pivotale, randomisierte, placebokontrollierte Phase-III-Studien untersuchten die Wirksamkeit, Reaktogenität und Sicherheit von $\mathrm{HZ} / \mathrm{su}$, verabreicht intramuskulär nach einem Schema von o und 2 Monaten. Abgefragte und nicht abgefragte unerwünschte Ereignisse (UEs) wurden für 7 bzw. 30 Tage nach jeder Dosis gesammelt; schwere UEs (SUEs) für ein Jahr nach der letzten Dosis; tödliche und verwandte SUEs und potenzielle immunvermittelte Krankheiten (pIMDs) während des gesamten Studiendauer. Die Reaktogenität wurde in einer Untergruppe von Teilnehmern bewertet; Die Sicherheit wurde bei allen geimpften Teilnehmern untersucht.

Ergebnisse: 29.305 Teilnehmer $\geq 50 \mathrm{~J}$ (HZ/su: 14.645; Placebo: 14.660) wurden in die gepoolte Analyse einbezogen. $\mathrm{HZ} / \mathrm{su}$ war reaktogener als Placebo. Die lokalen Reaktionen waren meist leicht bis mäßig und vorübergehend (mediane Dauer $=3$ Tage); Die Prozentsätze der Teilnehmer, die SAEs, tödliche SUEs und pIMDs berichteten, waren in beiden Kohorten am Tag 30 und ein Jahr nach der letzten Dosis vergleichbar. Die Prozentsätze der tödlichen SUE lagen zwischen 4,3\% (95\% Konfidenzintervall [KI]: 4,0-4,7) und 4,6\% (95\% Kl: 4,3-5,0), der pIMDs zwischen 1,2\% (95\% KI: 1,1-1,4) und 1,4\% (95\% -KI: 1,2-1,6), jeweils in der HZ/su- bzw. der Placebo-Gruppe. In beiden Kohorten waren Neoplasmen, Herzerkrankungen sowie Infektionen der Atemwege und parasitäre Erkrankungen die häufigsten Todesursachen. Die häufigsten pIMDs waren Polymyalgia rheumatica, rheumatoide Arthritis und Psoriasis.

Schlussfolgerung: Zusammen mit der hohen Wirksamkeit gegen $\mathrm{HZ}$ (97,2\% [95\% Kl: 93,7-99,0](1), 91,3\% [95\% KI: 86,8-94,5](2)) unterstützen die Sicherheitsdaten ein günstiges Nutzen-Risiko-Profil von $\mathrm{HZ} / \mathrm{su}$ in Teilnehmern $\geq 50$ Jahre.

(1) Lal H et al, N Engl J Med 2015; 372:2087-96.

(2) Cunningham AL et al, N Engl J Med 2016; 375:1019-32.

Finanzierung: GlaxoSmithKline Biologicals SA (NCT01165177; NCTo1165229)

\section{PS143}

Wirksamkeit, Immunogenität und Sicherheit eines adjuvantierten Herpes Zoster-Virus Untereinheiten-Impfstoffkandidaten bei Erwachsenen ab 60 Jahren und älter: Ergebnisse aus den Wirksamkeitsstudien ZOE-50 und ZOE-70

Janet McElhaney'; Himal Lal ${ }^{2}$; Anthony Cunningham ${ }^{3}$; Myron Levin ${ }^{4}$; Roman Chlibek ${ }^{5}$; Javier Diez-Domingo ; Eugene Athan ; J Johan Berglund ${ }^{8}$; Jackson Downey ${ }^{9}$; Wayne Ghesquiere ${ }^{10}$; Olivier Godeaux ${ }^{2}$; Iris Gorfinkel ${ }^{11}$; Shinn-Jang Hwang ${ }^{12}$; Tiina Korhonen ${ }^{13}$; Shelly McNeil ${ }^{14}$; Karlis Pauksens ${ }^{15}$; Joan Puig-Barbera ${ }^{6}$; Thiago Avelino-Silva ${ }^{16}$; Timo Vesikari ${ }^{13}$; Antonio Volpi ${ }^{17}$; Daisuke Watanabe ${ }^{18}$; Wilfried $\mathrm{Yeo}^{19}$; Laura Campora ${ }^{2}$; Carline Vanden Abeele ${ }^{2}$; Lidia Oostvogels ${ }^{2}$; Thomas Heinemann ${ }^{2}$; Johannes Hain ${ }^{20}$

${ }^{1}$ Health Sciences North Research Institute, Sudbury, ON, Canada; ${ }^{2}$ GlaxoSmithKline, King of Prussia, PA, United States; ${ }^{3}$ Westmeadand University of Sydney, Sydney, NSW, Australia; ${ }^{4}$ University of Colorado Anschutz Medical Campus, Aurora, CO, United States; ${ }^{5}$ University of Defence, Hradec Kralove, Czech Republic; ${ }^{6}$ Fundación para el Fomentode la Investigación Sanitaria y Biomédica (FISABIO), Valencia, Spain; ${ }^{7}$ BarwonHealth, Deakin University, Geelong, VIC, Australia; ${ }^{8}$ Blekinge Institute of Technology, Karlskrona, Sweden; ${ }^{9}$ Jacksonville Center for Clinical Research, Jacksonville, FL, United States; ${ }^{10}$ University of British Colombia, Victoria, BC, Canada; ${ }^{11}$ Prime Health Clinical Research, Toronto, ON, Canada; ${ }^{12}$ Taipei Veterans General Hospital, Taipei, Taiwan; ${ }^{13}$ University of Tampere, Tampere, Finland; ${ }^{14}$ IWK Health Centre and Nova Scotia Health Authority, Halifax, NS, Canada; ${ }^{15}$ Uppsala University Hospital, Uppsala, Finland; ${ }^{16}$ University of Sao Paulo Medical School, Sao Paulo, Brazil; ${ }^{17}$ University of Rome Tor Vergata, Rome, Italy; ${ }^{18}$ Aichi Medical University, Nagakute, Japan; ${ }^{19}$ University of Wollongong, Wollongong, NSW, Australia; ${ }^{20} \mathrm{GlaxoSmithKline} \mathrm{GmbH} \mathrm{\&} \mathrm{Co.} \mathrm{KG,} \mathrm{München}$

Hintergrund: In den zwei Phase III Zulassungsstudien (ZOE-50, ZOE70 [NCT01165177, NCT01165229]) zeigte das rekombinante Herpes Zoster $(\mathrm{HZ})$ Virus Untereinheit Vakzin (HZ/su) eine Wirksamkeit von über $90 \%$ gegenüber $\mathrm{HZ}$ bei älteren Erwachsenen. Wir berichten über die Wirksamkeit (VE) von $\mathrm{HZ} / \mathrm{su}$ gegen $\mathrm{HZ}$ und post-Zoster Neuralgie (PZN), Immunogenität- und Sicherheitsdaten bei Erwachsenen mit 60 Jahren (J) und älter, (VE $\geq 60 \mathrm{~J}$ [ZOE-50], Immunogenität/Sicherheit 60$69 \mathrm{~J}$ [ZOE-50], Immunogenität/Sicherheit $\geq 70 \mathrm{~J}$ [gepoolt ZOE-50/70]).

Methoden: Die Studienteilnehmer wurden 1:1 randomisiert und erhielten 2 intramuskuläre Injektionen in einem Abstand von 2 Monaten (M) entweder mit dem HZ/su Vakzine (bestehend aus $50 \mu \mathrm{g}$ Varizella-Zoster Virus Glycoprotein E [gE] und dem Adjuvants (ASo1B) oder Plazebo (Natriumchlorid Lösung). Die VE wurde anhand der $\mathrm{HZ}$ - und PZN-Fälle, die humorale Immunantwort durch anti-gE ELISA vor der Injektion, 1, 12, 24 und $36 \mathrm{M}$ nach der zweiten Injektion (Untergruppe) im Vergleich zu Placebo ermittelt. Die Sicherheit wurde mittels abgefragter unerwünschter Ereignisse (Tagebucheinträge einer Untergruppe über 7 Tage nach Impfung) und nicht-abgefragte unerwünschte Ereignissen für 30 Tage nach jeder Injektionerhoben; , Unerwünschte Ereignisse, die medizinisch behandelt werden mussten (MAEs) wurden über $6 \mathrm{M}$ nach der 2 Injektion erfasst, tödliche Ereignisse, schwerwiegende AEs (SAEs) in Verbindung zur Impfung/ Studienteilnahme, potentiell immun-vermittelte Krankheiten (pIMDs) wurden bis zum Studienende erfasst..

Ergebnisse: Es wurden 4490 Teilnehmer 60-69 J (HZ/su: 2244; Placebo: 2246), $8122 \geq 60$ (4053; 4069) aus ZOE-50 und insgesamt $17531 \geq$ $70 \mathrm{~J}(8758 ; 8773)$ gepoolt aus beiden Studien geimpft. VE gegen $\mathrm{HZ}$ in ZOE-50 war 97,6\% (95\% Konfidenzinterwall: 92,7-99,6) bei Erwachsenen $\geq 60 \mathrm{~J}$. VE bei 60-69 J war $97,4 \%$ (ZOE-50) und bei $\geq 70 \mathrm{~J} 91,3 \%$ (gepoolt ZOE-50/70). VE gegen PZN war 100,0\% (55,2 - 100,0) bei Erwachsenen $\geq 60$ (ZOE-50) und $88,8 \%$ bei den $\geq 70 \mathrm{~J}$ (gepoolt ZOE-50/70). Die höchste Rate der Immunantwort und die geometrisch mittlere Konzentration von anti-gE Antikörpern in der HZ/su Kohorte wurde einen $M$ nach der zweiten Injektion gemessen. Diese Immunantwort 
hielt über $36 \mathrm{M}$. HZ/su zeigte mehr Reaktogenität auf als Placebo, wobei die Reaktionen meist mild bis moderat und von kurzer Dauer waren. SAEs, tödliche Fälle und pIMDs waren mit der Placebo Gruppe vergleichbar.

Schlussfolgerung: Das HZ/su Vakzin zeigt eine in Personen $\geq 60$ Jahren eine hohe Wirksamkeit gegen $\mathrm{HZ}$ und $\mathrm{PZN}$, ist hoch immunogen und hat einm gutes Sicherheitsprofil.

\section{Finanzierung: GlaxoSmithKline Biologicals SA (NCT01165177; NCTo1165229) \\ PS144 \\ Die nicht-interventionelle PEARL Studie zur Verwendung von Alirocumab im klinischen Alltag in Deutschland}

\author{
Klaus G. Parhofer ${ }^{1}$; Berndt von Stritzky²; W Dieter Paar ${ }^{2}$
}

${ }^{1}$ Medizinische Klinik II - Grosshadern, Klinikum der Universität München, München; ${ }^{2}$ Sanofi-Aventis Deutschland $\mathrm{GmbH}$, Berlin

Hintergrund: Die ESC/EAS Task Force gibt an, dass PCSK9-Inhibitoren bei Patienten eingesetzt werden können, die bei nachgewiesener Atheroskleroseerkrankung nicht adäquat mit Statin und Ezetimib behandelt sind.

Ziel: Die PEARL (Prospektive Nicht-Interventionelle Studie zur Erfassung der WirksAmkeit und VeRträgLichkeit des PCSK9-Inhibitors PRALUENT) Studie erfasste die Wirksamkeit und Sicherheit des PCSK9-Inhibitors Alirocumab bei Patienten mit Hypercholesterinämie im klinischen Alltag.

Methoden: PEARL war eine offene, prospektive, nicht-interventionelle, Multi-Center Studie in Deutschland, in welche Patienten $(n=619)$ eingeschlossen wurden, die trotz maximal tolerierter lipidsenkender Therapie (außer PCSK9-Inhibitoren) ein erhöhtes LDL-Cholesterin (LDL-C) aufwiesen (>1,81 oder 2,59 mmol/l [70 oder $100 \mathrm{mg} / \mathrm{dl}$ ] abhängig vom kardiovaskulären Risiko) und $\geq 1$ Dosis Alirocumab 75 oder 150 mg (alle 2 Wochen [Q2W]) erhielten. Die Beobachtungszeit betrug 24 Wochen, während dessen die Alirocumab-Dosis anpasst werden konnte. Der primäre Zielparameter war die Reduktion des LDL-C mit Alirocumab nach 24 Wochen.

Ergebnisse: $64,4 \%$ der eingeschlossenen Patienten waren männlich das Durchschnittsalter betrug 61,6 Jahre. 90,2\% hatten eine kardiovaskuläre Erkrankung, einschließlich koronare Herzkrankheit $(72,4 \%)$, cerebrovaskuläre Erkrankung (9,2\%) und periphere arterielle Verschlusskrankheit (13,2\%). Eine familiäre Hypercholesterinämie wiesen 50,8\% und einen Diabetes $27,6 \%$ auf. $45,3 \%$ der Patienten waren komplett Statin-intolerant ( $\geq 2$ Statine nicht vertragen) und $27,6 \%$ wiesen eine partielle Statinintoleranz auf (zur Zielwerterreichung ausreichende Statindosis nicht toleriert). Vor der ersten Alirocumab-Dosis erhielten 23,5\% der Patienten nur Statins, 47,8\% eine Kombination Statin mit anderen Lipidsenkern (Ezetimib, Fibrat und/oder Anionenaustauscherharz), 10,1\% eine nicht-Statin-lipidsenkende Therapie und 1,9\% erhielten andere Lipidsenker; für $16,7 \%$ lag keine Information vor. Zu Beginn der Alirocumabtherapie lag das LDL-C bei $4,7 \pm 1,6 \mathrm{mmol} / \mathrm{l}(180,5 \pm 60,7$ $\mathrm{mg} / \mathrm{dl}$ ). Bei Woche $24 \mathrm{lag}$ das durchschnittliche LDL-C bei $2,3 \pm 1,2$ $\mathrm{mmol} / \mathrm{l}(89,8 \pm 46,0 \mathrm{mg} / \mathrm{dl})$. Die prozentuale Absenkung (least-squares mean percentage change) vom Ausgangswert lag bei $-48,6 \%$ bei Woche 24 (Konfidenzintervall: $-50,7 ;-46,5$ ). Bei $72,9 \%$ war die initiale Alirocumab-Dosis $75 \mathrm{mg}$ und bei $24,5 \% 150 \mathrm{mg} \mathrm{Q} 2 \mathrm{~W}$. Bei $20,4 \%$ wurde die Alirocumab-Dosis von $75 \mathrm{mg}$ auf $150 \mathrm{mg} \mathrm{Q} 2 \mathrm{~W}$ gesteigert, bei 4,0\% wurde die Dosis reduziert. Insgesamt unterbrachen $13,4 \%$ der Patienten die Alirocumab-Therapie. Therapie-assoziierte Wirkungen wurden bei 10,3\% der Patienten berichtet, wobei Myalgien mit 7,3\% am häufigsten auftraten.

Schlussfolgerung: Diese Daten aus dem klinischen Alltag zeigen, dass Alirocumab vor allem bei Patienten mit hohen kardiovaskulären Risiko und kompletter oder partieller Statin-Intoleranz eingesetzt wird. Die beobachtete Wirksamkeit und Sicherheit deckt sich mit den im ODYSSEY Phase III Programm beschriebenen Daten.

\section{PS145}

Erste klinische Erfahrungen mit den PCSK9-Hemmern Evolocumab und Alirozumab zur LDL und $L p(a)$ Reduktion bei Patienten mit familiärer Hypercholesterinämie oder manifester Atherosklerose-Progression

Madan Raj Poudel'; Dana Stoyanova'; Regine Gottfried'; Dieter Horstkotte'; Frank Van Buuren²; Klaus-Peter Mellwig'

${ }^{1}$ HDZ NRW, Bad Oeynhausen; ${ }^{2}$ St. Martinus-Hospital, Olpe; ${ }^{1}$ HDZ NRW, Bad Oeynhausen

Fragestellung: „Werden Hochrisiko Patienten unter der Therapie mit proprotein convertase subtilisin/kexin type 9 (PCSK9)-Hemmer die von den ESC Leitlinien empfohlenen Zielwerte für Low density Lipoprotein (LDL) und Lipoprotein(a) Lp(a) erreicht?".

Methodik: Es wurden 27 Patienten aus der Lipidambulanz der kardiologischen Klinik des HDZ NRW mit manifester Atherosklerose (KHK, pAVK, Karotisstenose) oder familiärer Hypercholesterinämie eingeschlossen, die die Kriterien für eine Therapie mit PCSKg Hemmer erfüllt haben (unzureichende LDL Senkung unter Statin-Ezetimibtherapie oder Statin-Ezetimib-Unverträglichkeit). Das Patientenkollektiv wurde nach dem Therapiebeginn weitere 20 Wochen verfolgt. (Regelmäßige Bestimmung der Lipidparameter, alle 2 Wochen).

Ergebnisse: Die Patienten wiesen ein mittleres Alter von $61,3 \pm 11,3$ Jahr (J3-77) auf; (48,1\% Männer) 21 Pat. mit manifester KHK, 19 Pat. mit nachgewiesenem Progress der KHK, 2 Pat mit pAVK, 5 Pat mit extrakranieller Carotisstenose; 3 Pat. mit familiärer Hypercholesterinämie ohne manifeste Atherosklerose. Bei 22 Patienten wurde eine Revaskularisation durchgeführt, bei 21 Pat. eine perkutane transluminale koronare Angioplastie (PTCA), und bei 4 Pat eine Coronararterienbypassgraft-Operation (CABG). Eine Statine und oder eine Ezetimibtherapie zu Beginn der Beobachtung wiesen 33,3\% bzw. 25,9\% der Patienten $(n=9 / 7)$ auf, bei denen jedoch der Zielwert über den von European Society of Cardiology (ESC) empfohlenen Wert lag. Bei den Übrigen Patienten bestand eine Statin/Ezetimib-Unverträglichkeit. Vor der Einleitung der Therapie mit PCSK9-Hemmer betrug die LDL Konzentration $168,63 \pm 79,89 \mathrm{mg} / \mathrm{dl}$. Unter der Therapie mit Alirocumab $(n=21)$ und Evolocumab $(n=6)$ wurde eine LDL-Senkung im Mittel auf $85,68 \pm 62,69 \mathrm{mg} / \mathrm{dl},(\mathrm{p}=0.0001)$ erzielt. Die statisch relevante maximale LDL-Senkung konnte bereits nach 4 Wochen beobachtet werden $(79,96 \pm 44,45) \mathrm{mg} / \mathrm{dl}$. In den nachfolgenden 16 Wochen konnte keine weitere statistisch relevante LDL Senkung dokumentiert werden $(p$ $=0.32$ ). Unter der Therapie mit PCSK9 Hemmer konnte keine statistisch signifikante $L p(a)$ Senkung beobachtet werden. Vor der Therapie lag der $L p(a)$ Wert bei $25,92 \%(n=7)$ der Patienten über $60 \mathrm{mg} / \mathrm{dl}$ $(89,50 \pm .13,56)$. Am Ende der Beobachtung konnte eine statistisch nicht signifikante Senkung des $L p(a)$ von $13,40 \%(p=0.17)$ gezeigt werden.

Schlussfolgerung: Unter der PCSK9 Hemmer Therapie konnte bereits nach 4 Wochen eine statistisch signifikante LDL-Senkung (in Woche 8 maximale Senkung) erzielt werden. Bei den Hoch-Risikopatienten wurde der von den ESC empfohlene LDL-Zielwert unter 7omg/ dl trotz PCSK9 Hemmer nicht erreicht. Für dieses Patientenkollektiv ist die Indikation der Lipidaphrese zu diskutieren. Für die Patienten 
mit hohem Lp(a) über 6omg/dl konnte unter der Therapie mit PCSKg-Hemmer keine ausreichende Senkung beobachtet werden. Bei Progress der Atherosklerose ist bei diesem Patientenkollektiv ebenfalls eine Lipidaphrese zu diskutieren.

\section{PS146}

Machbarkeit und Wirksamkeit von hochintensivem Intervallausdauertraining (HIIT) bei Tumorpatienten: Eine systematische Übersichtsarbeit mit Meta-Analyse

Dejan Reljic; Hans Joachim Herrmann; Markus Neurath; Yurdagül Zopf

Universitätsklinikum Erlangen - Medizinische Klinik 1, Erlangen

Hintergrund: Tumorpatienten weisen ein erhöhtes Risiko fü Herz-Kreislauferkrankungen auf, was unter anderem auf kardiotoxische Effekte bestimmter Krebstherapien sowie auf die häufig reduzierte körperliche Aktivität in Folge der Erkrankung zurückgeführt wird. Eine geringe kardiorespiratorische Fitness ist zudem mit einem erhöhten Risiko für postoperative Komplikationen und einer insgesamt schlechteren Langzeitprognose bei Tumorerkrankungen assoziiert. Regelmäßiges Ausdauertraining hat somit einen hohen Stellenwert im Rahmen der supportiven Krebstherapie. Hochintensives Intervallausdauertraining (HIIT) ist eine spezifische Form des Ausdauertrainings, mit der sich die Fitness Studien zufolge besonders effektiv und zeiteffizient verbessern lässt. Bisher gibt es jedoch nur sehr wenige Daten zur Anwendung von HIIT bei Krebserkrankungen. In der vorliegenden Übersichtsarbeit sollten daher die Machbarkeit und Effektivität von HIIT bei Tumorpatienten evaluiert werden.

Methodik: Die Datenbank Pubmed/MEDLINE wurde systematisch nach relevanten Artikeln, die bis Januar 2018 publiziert wurden, durchsucht. Die Einschlusskriterien waren: 1)Strukturierte HIIT-Studien mit Tumorpatienten; 2)Erfassung von Compliance und unerwünschten Ereignissen; 3)Erfassung der Effekte auf die maximalen Sauerstoffaufnahme (VO2max); 4)Veröffentlichung in einem peer-reviewed Journal.

Ergebnisse: Insgesamt wurden 292 Artikel gefunden, von denen 7 ( $n=197$ Probanden mit unterschiedlichen Krebsentitäten, Alter: 57.2 \pm 6.9 Jahre) die Einschlusskriterien erfüllten. Die Interventionen dauerten im Mittel 8 Wochen, wobei durchschnittlich 3x/Woche trainiert wurde. Die mittlere Belastungsintensität betrug $91 \pm 2.9 \%$ der maximalen Herzfrequenz. Im Mittel dauerte das Training $32 \pm 5$ Min./ Einheit. Insgesamt zeigten sich in den Studien eine hohe Compliance (92\%) und niedrige Dropout-Raten (4\%). In keiner der Studien wurden schwerwiegende Vorfälle verzeichnet. Insgesamt wurden nur 3 geringfügige Nebeneffekte (Ischiasschmerzen $n=1$; Knieschmerzen $n=2$ ) berichtet. Die gepoolte Steigerung der VO2max betrug $3.5 \mathrm{ml} / \mathrm{kg} / \mathrm{min}$.

Schlussfolgerung: Die Ergebnisse dieser systematischen Übersichtsarbeit weisen darauf hin, dass sich mit HIIT innerhalb weniger Wochen effektiv die Fitness bei Tumorpatienten steigern lassen kann. Eine Verbesserung der VO2max um $3.5 \mathrm{ml} / \mathrm{kg} / \mathrm{min}$ (=1 Metabolische Einheit, $M E T$ ) ist klinisch bedeutsam, da eine Steigerung des Fitnessgrades um 1 MET Untersuchungen zufolge mit einer Reduktion des Gesamtmortalitätsrisikos um 12\% assoziiert ist. Zudem zeigte sich in den Studien trotz der im Vergleich zu herkömmlichem Ausdauertraining deutlich höheren Belastungsintensitäten eine gute Verträglichkeit und Akzeptanz von HIIT. HIIT könnte somit eine vielversprechende Option für die Sporttherapie mit Tumorpatienten darstellen. Aufgrund der geringen Studienzahl sind jedoch weitere Untersuchungen notwendig, um diese ersten Ergebnisse zu untermauern.

\section{PS147}

Effekte von zeiteffizientem, hochintensivem Intervallausdauertraining auf die kardiorespiratorische Fitness und kardiometabolische Risikomarker in einem Real-World-Setting: Eine randomisiert-kontrollierte Pilotstudie

Dejan Reljic'; Felix Wittmann²; Joachim Fischer²

'Universitätsklinikum Erlangen - Medizinische Klinik 1, Erlangen

${ }^{2}$ Medizinische Fakultät Mannheim der Universität Heidelberg, Mannheim

Hintergrund: Zunehmender Bewegungsmangel in der Bevölkerung und ein alarmierender Anstieg an mit Inaktivität assoziierten Erkrankungen erfordern die dringende Entwicklung und Evaluation innovativer Bewegungskonzepte. Erwachsenen werden mindestens $150 \mathrm{Min}$. körperliche Aktivität empfohlen, um gesundheitsprotektive Effekte zu erzielen. Ein Großteil der Bevölkerung erreicht diese Empfehlung jedoch nicht. Dabei zählt vor allem Zeitmangel als eine der häufigsten Ursachen für unzureichende Bewegung. Aktuell findet hochintensives Intervallausdauertraining (HIIT) als potentiell zeiteffiziente und effektive Trainingsmethode zur Gesundheitsförderung zunehmend Beachtung. Bisherige HIIT-Studien wurden jedoch vorwiegend unter Laborbedingungen durchgeführt, sodass die praktische Umsetzung und die Effekte von HIIT unter authentischen („Real-World“) Bedingungen noch unzureichend untersucht sind. Zudem wurde besonders zeiteffizientes HIIT bisher nur anhand sehr extremer, supramaximaler Protokolle getestet, deren Umsetzbarkeit bei Untrainierten fraglich ist. Ziel dieser Studie war es daher, die Machbarkeit und die Effekte von zwei praktikableren, zeiteffizienten HIIT-Protokollen ( $\leq$ 30 Min. Zeitaufwand/Woche) in einem öffentlichen Fitnessstudio zu untersuchen.

Methoden: Es wurden 34 untrainierte Männer und Frauen randomisiert entweder A)einem 2X4 Min. HIIT (2X4-HIIT), B)5X1 Min. HIIT (5X1-HIIT) (jeweils mit einer Intensität von $85-95 \%$ der maximalen Herzfrequenz, HFmax) oder C)einer aktiven Kontrollgruppe zugeteilt, die ein moderat-intensives, kontinuierliches Ausdauertraining (MIKT, 38 Min./Trainingseinheit, $65-75 \%$ HFmax) durchführte. Alle Trainings wurden 2x/Woche in Form von Gruppenkursen auf Spinning-Bikes über 8 Wochen absolviert. Zu Beginn und am Ende der Intervention wurden die maximale Sauerstoffaufnahme ( $\mathrm{VO}_{2} \mathrm{max}$ ), kardiometabolische Risikomarker und subjektive Parameter erfasst.

Ergebnisse: Alle Trainingsprotokolle wurden gut toleriert und es traten keine unerwünschten Vorfälle auf. Die Dropout-Raten waren bei $2 \times 4-\mathrm{HIIT}(17 \%)$ und 5X1-HIIT (8\%) geringer als bei MIKT (30\%). Die $\mathrm{VO}_{2 m a x}$ konnte mit allen Protokollen signifikant gesteigert werden, jedoch waren die Effekte mit $2 \times 4-\mathrm{HIIT}(+20 \%, \mathrm{P}<0.01)$ und $5 \times 1-\mathrm{HIIT}$ $(+27 \%, \mathrm{P}<0.001)$ größer, als mit MIKT $(+16 \%, \mathrm{P}<0.05)$. Das Cholesterinprofil konnte mit allen Protokollen verbessert werden. Nur mit 2X4-HIIT zeigte sich aber eine Verringerung des Metabolischen Syndrom Z-Scores $(P<0.05)$ und nur mit 5x1-HIIT konnte der Taillenumfang signifikant verringert $(P<0.05)$ sowie die subjektive Arbeitsfähigkeit gesteigert werden $(P<0.05)$.

Schlussfolgerung: Die Ergebnisse dieser Pilotstudie zeigen, dass sich zeiteffizientes HIIT praktikabel in einem Real-World-Setting umsetzen lässt. Die vorliegenden Daten deuten zudem darauf hin, dass mit HIIT, sowohl in Form von 2X4-HIIT oder als 5X1-HIIT, bereits mit einem Zeitaufwand von nur 30 Min./Woche signifikante Verbesserungen der $\mathrm{VO}_{2}$ max und kardiometabolischer Risikomarker erzielen werden können. 


\section{PS148}

Ganzkörper-Elektromyostimulation kombiniert mit individualisiertem Ernährungssupport verbessert die Körperzusammensetzung von Patienten mit fortgeschrittenen Krebserkrankungen

Kristin Schink'; Hans Joachim Herrmann'; Raphaela Schwappacher'; Till Orlemann'; Julia Meyer'; Elisabeth Waldmann ${ }^{2}$; Bernd Wullich'; Andreas Kahlmeyer'; Rainer Fietkau'; Dorota Lubgan'; Matthias W. Beckmann'; Carolin Hack'; Wolfgang Kemmler'; Jürgen Siebler'; Markus F. Neurath'; Yurdagül Zopf ${ }^{1}$

${ }^{1}$ Universitätsklinikum Erlangen, Falkenstein; ${ }^{2}$ Friedrich-Alexander-Universität Erlangen-Nürnberg, Erlangen

Hintergrund: Als Hauptmerkmal der Tumorkachexie besitzt der Abbau der Skelettmuskelmasse (SMM) einen bedeutenden Einfluss auf die Lebensqualität, Therapieverträglichkeit und Prognose von Tumorpatienten. Einzelne bewegungs- und ernährungstherapeutische Maßnahmen zeigten positive Effekte auf den Erhalt des Körpergewichts und der Lebensqualität von Krebspatienten; allerdings nur mit marginalem Erfolg auf die SMM. Insbesondere Patienten in fortgeschrittenen Krebsstadien sind während ihrer onkologischen Behandlung häufig zu schwach um ein konventionelles intensives Muskeltraining durchzuführen. In der vorliegenden Pilotstudie sollte daher der Effekt eines kombinierten Ansatzes aus der neuartigen Trainingsmethode der Ganzkörper-Elektromyostimulation (WB-EMS) und einer individuell angepassten, proteinreichen Ernährung auf diese Patientengruppe untersucht werden.

Methoden: In einer prospektiven, kontrollierten Studie wurden $\mathrm{Pa}-$ tienten mit fortgeschrittenen soliden Tumoren (56.5 \% männlich; 59.9 \pm 12.7 Jahre; $\mathrm{N}=131$ ) unter onkologischer Therapie in eine Kontrollgruppe $(n=35)$ mit routinemäßiger eiweißreicher Ernährungsunterstützung (Proteinzufuhr $>1.0 \mathrm{~g} / \mathrm{kg} / \mathrm{Tag}$ ) oder in eine Interventionsgruppe $(n=96)$ mit einem regelmäßigen WB-EMS Training ( $2 x /$ Woche á $20 \mathrm{~min}$ ) über einen Studienzeitraum von 12 Wochen eingeteilt. Primärer Endpunkt war die Veränderung der SMM. SMM, Körperzusammensetzung und Körpergewicht wurden mit Hilfe der Bioelektrischen Impedanzanalyse zu Beginn, nach 4, 8 und 12 Wochen bestimmt. Die körperliche Funktionalität wurde alle 4 Wochen durch ein Hand Dynamometer sowie zu Beginn und Studienende durch den 6-Minuten-Gehtest und Karnofsky-Leistungsindex erfasst. Im gleichen Zeitraum erfolgte die Bestimmung der inflammatorischen Blutparameter (Albumin, CRP) sowie der Lebensqualität und Fatigue durch den EORTC QLQ-C30 und FACIT-Fatigue Scale Fragebogen.

Ergebnisse: 82 Patienten beendeten die Studie. Es traten keine WB-EMS-bedingten negativen Nebenwirkungen auf. Nach 12 Wochen wiesen die Patienten der WB-EMS Gruppe gegenüber den Kontrollpatienten eine signifikant höhere SMM (0.53 kg [0.08, 0.98]; $p=0.022)$ und Körpergewicht $(1.02 \mathrm{~kg}[0.05,1.98] ; p=0.039)$ auf. Außerdem konnte eine signifikante Verbesserung der 6-Minuten-Gehstrecke und des Leistungsstatus der WB-EMS Gruppe gegenüber den Kontrollen beobachtet werden $(p \leq 0.05)$. Es konnten keine signifikanten Gruppenunterschiede in der Handkraft, Lebensqualität, Fatigue und den Blutparametern festgestellt werden.

Zusammenfassung: WB-EMS kombiniert mit einer eiweißreichen Ernährungstherapie ist ein geeigneter und effektiver supportiver Therapieansatz um dem tumorbedingten Muskelabbau in Patienten mit fortgeschrittenem Tumorleiden während der onkologischen Therapie entgegenzuwirken und die körperliche Funktionalität zu steigern. Der Einfluss auf die Lebensqualität und das Überleben innerhalb der verschiedenen Tumorentitäten sollte in zukünftigen Studien untersucht werden.
PS149

Exercise training as secondary prevention of endothelial dysfunction in ZSF-1 animals with HFpEF

Zelia Schmederer'; Natale Rolim²; T Scott Bowen ${ }^{3}$; Axel Linke'; Ulrik Wisloff ${ }^{2}$; Volker Adams ${ }^{5}$

${ }^{1}$ University Leipzig, Heart Center Leipzig, Leipzig; ${ }^{2}$ K.G. Jebsen Center of Exercise in Medicine, NTNU, Trondheim, Norway; ${ }^{3}$ University of Leeds, Leeds, United Kingdom; ${ }^{4} \mathrm{TU}$ Dresden, Heart Center Dresden, Dresden; ${ }^{5}$ TU Dresden, Heart Center Dresden - on behalf of the OptimEx study group, Dresden

Background: Endothelial dysfunction seems to be a pivotal player in the comorbidity-driven pathogenesis and the prognosis of heart failure with preserved left ventricular ejection fraction (HFpEF). Despite normal left ventricular ejection fraction (LVEF), patients with HFpEF present exercise intolerance which can be attenuated by exercise training. Whether exercise training and which optimal modality improves endothelial function after disease onset is still unknown. Alterations in the vascular system have been described with conflicting results and the molecular mechanisms mediating the beneficial effects of exercise training after disease onset is still unknown.

Aims: The present study, therefore, used a cardiometabolic rat model to further assess functional, molecular and histological alterations in the vascular system induced by HFpEF and to evaluate the potentially beneficial effects of exercise training of different modalities in secondary prevention.

Protocol: At the age of 20 weeks, obese Zucker diabetic fatty/spontaneously hypertensive heart failure $\mathrm{F}_{1}$ hybrid (ZSF1) rats with leptin resistance $(n=12)$ were compared to their lean counterparts $(n=8)$. Further 3 groups of obese ZSF1 rats were assessed 8 weeks later at the age of 28 weeks following sedentary behavior $(n=15)$, moderate-continuous training (MCT; $n=11)$ or high-intensity interval training (HIIT; $n=11)$.

Results: Obese rats displayed diastolic dysfunction, LV hypertrophy, exercise intolerance $(p<0.05)$ and preserved LVEF, meeting the criteria for HFpEF. These animals showed impaired endothelial-dependent $(p<0.001)$ and -independent $(p<0.05)$ vasodilation of the thoracic descending aorta, compared with controls. This functional impairment was associated with reduced expression and activation of the main NO generating enzyme eNOS ( $p<0.05)$, an elevated expression of the ROS generating enzyme NADPH oxidase gp91phox $(p<0.05)$, and reduced endothelial cell coverage $(p<0.001)$. Also, a reduced I/III ratio of collagen transcription $(p<0.05)$, aortic hypertrophy $(p<0.05)$, increased expression of the protein-crosslinking enzyme LOX-1 $(p=0.07)$, and higher transcription of the proinflammatory cytokine TNF-alpha $(p=0.09)$ were observed in the obese animals. Exercise, independent of modality, improved endothelial function, which was associated with a reversion of all molecular parameters except eNOS.

Conclusion: A cardiometabolic obese rat model of HFpEF revealed impaired endothelial function. This was associated with molecular and histological alterations, including reduced expression and activation of eNOS, as well as increased oxidative stress and inflammation, resulting in reduced NO bioavailability and increased vascular stiffness. Exercise training (independent of protocol) reversed these alterations via mechanisms including all the above-mentioned parameters but eNOS. 


\section{PS150 Schwierige HCC-Sekundäreprophylaxe bei Peliose und Leberzirrhose Child A bei hypoplastischer Pfortader}

Jessica Weis; Sophie Schlosser; Martina Müller

Universitätsklinikum Regensburg, Regensburg

Hintergrund: Die Peliosis hepatis ist eine seltene Erkrankung, die unabhängig von Alter und Geschlecht auftritt und durch Proliferation von Lebersinusoiden zu multiplen wenige Milimeter bis $4 \mathrm{~cm}$ großen blutgefüllten Zysten im Leberparenchym führt. Die Erkrankung kann fokal oder ubiquitär in der Leber auftreten und ist manchmal auch extrahepatisch u.a. in Lungen, Nieren, Darm und Lymphknoten zu finden. Die Ätiologie ist weitestgehend unklar. Verschiedene Faktoren wie Medikamente (z.B. Steroide, orale Kontrazeptiva, Azathioprin, Methotrexat), Infektionen (z.B. HIV, Bartonella henselae, Tuberkulose), Autoimmunerkrankungen und maligne Erkrankungen (z.B. HCC, Myelodysplastisches Syndrom, Morbus Castleman) sind mit der Erkrankung assoziiert. Meist ist die Peliose ein Zufallsbefund, da sie in der Regel asymptomatisch verläuft. Wenige Patienten stellen sich mit abdominellen Schmerzen, Ikterus, Hepatomegalie, portaler Hypertension oder akutem Leberversagen vor. In bis zu drei Viertel der Fälle kann es abhängig von der Lokalisation zu einer lebensbedrohlichen Leberruptur kommen.

Fallbericht: 2003 wurde bei einem 14-jährigen männlichen Patienten bei Ikterus und Gynäkomastie eine Leberzirrhose Child A bei hypoplastischer Pfortader diagnostiziert. Bei Ösophagusvarizen erfolgten mehrfach Ligaturen. Die Leberfunktion blieb stabil. Halbjährlich wurden Sonographien zur Früherkennung eines Hepatozellulären Karzinoms (HCC) durchgeführt. 2012 wurden hierbei erstmalig zwei Leberraumforderungen in Lebersegment VII und VIII festgestellt. Ein MRT der Leber ergab weitere Raumforderungen, welche bildmorphologisch mit einem HCC oder Cholangiokarzinom (CCC) vereinbar waren. Bioptisch konnte ein Malignom ausgeschlossen werden. Es zeigte sich eine Peliose hepatis. Ein Jahr später erfolgte bei Größenprogredienz der Raumforderungen erneut eine Leberbiopsie, die abermals ein Malignom ausschließen konnte. Bisher konnte bei stabiler Peliose kein Hepatozelluläres Karzinom nachgewiesen werden.

Schlussfolgerung: Der vorliegende Fall beschreibt einen Langzeitverlauf einer Peliosis hepatis. Er zeigt, wie schwierig die Diagnosestellung und die Abgrenzung zum HCC mittels bildmorphologischer Diagnostik ist. Letztendlich kann nur mittels Punktion und histopathologischer Untersuchung die definitive Diagnose gestellt werden. Dies erschwert das Follow-up dieser Patienten, die aufgrund der Assoziation der Peliose mit malignen Erkrankungen ein enges Monitoring erhalten sollten. Wiederholt können Biopsien erforderlich werden.

\section{Versorgungsstrukturen - Versorgungsforschung I}

\section{PS152}

Allgemein- und viszeralchirurgische Oberarzt(OA)-Konsile für andere medizinische Disziplinen über 10 Jahre an einem tertiären Zentrum - Klinisches Befund-, Diagnose- und therapeutisches Entscheidungsspektrum und Einflussfaktoren (Ergebnisse einer klinisch

Joul Deeb; Manuela Petersen; Siegfried Kropf; Frank Meyer

Universitätsklinikum Magdeburg A.ö.R., Magdeburg

Ziel: Analyse der Breite variabler Diagnosen \& des Profils der chir. Entsch.-Findung mit weiterf., ggf. op. Maßnahmen im Rahmen des allg.- \& visz.-chir. Oberarzt-Konsils für med. Nachbardisziplinen über einen def. Beob.-Zeitraum an einer repräsentativen Pat.-Klientel in einem klin. Zentrum der Max.-Versorgung.

Patienten und Methode: Über 10 Jahre wurden alle konsekutiven Patienten im Rahmen einer klin.-systemat., prospektiven unizentr. Observationsstudie (Registration trial DRKSooo11888 on DRKS) an einem tertiären Zentrum in einer computer-basierten Patientendatei erfasst und hinsichtlich klin. Befund-, Diagnose- \& therapeut.. Entsch.-Spektrum (u.a. Übereinstimmungsrate von Verdachts- \& def. Diagnose, Rate der gestellten OP-Indikation u.a.) \& ihrer signifik.. Einflussfaktoren sowie hinsichtlich zeitabhängiger Entwicklungstrends mittels $x^{2}-\&$ U-Test analysiert $(p<0,05)$.

Ergebnisse (Eckpunkte): - Vom 01.10.2006-30.09.2016 wurden insgesamt 549 Fälle dokumentiert (Geschl.-Verhältnis: $m: f=321: 228$ [1,41:1]; Durchschnittsalter: 63 [Streubreite: 15-98] Jahre).

- Die dominierende Fachdisziplin war die Kardiologie $(n=109 / 549 ;$ $19,9 \%)$, gefolgt von anderen chir. Fächern ( $n=65 / 549 ; 11,8 \%) \&$ der Gastroenterologie $(n=62 / 549 ; 11,3 \%)$.

- Das Spektrum der Konsultationsgründe umfasste: A) eher klin.-beurteilend geprägt: „Mitbeurteilung" zu 69,8\% ( $n=383 / 549)$, "Therapieempfehlung" $14,4 \%$ ( $n=79 / 549)$, „Verlaufskontrollen" $16 \%(n=88 / 549)$, „Wiedervorstellungen“ 2,4\% ( $n=13 / 549)$, B) Operations(OP)-assoziiert: Frage der OP-Indikation $24,4 \%(n=134 / 549)$ \& postop. Wiedervorstellungen $6,9 \%(n=38 / 549)$.

- Das Diagnoseprofil wurde von Wundheilungsstörungen ( $n=39 / 549$; 7,1\%) bestimmt.

- Bei 13,3\% ( $n=73 / 549)$ der Patienten wurde die unmittelbare OP-Indikation gestellt \& bei 10,6\% ( $n=58 / 549)$ wurde die OP elektiv empfohlen.

- Die Übereinstimmungsrate von Verdachts- \& definitiver Diagnose lag bei lediglich $55,2 \%(n=187 / 339)$.

- Im Geschlechtervergleich wurde bezüglich des def. Diagnosespektrums bei Frauen häufiger eine Ischämie $(n=9 / 13)$ diagnostiziert $(p=0,048)$.

- Im Vergleich der Altersgruppen bestand bei Patienten ab 66 Jahre $(n=280)$ ein höheres Risiko, eine manifeste Pneumonie $(n=9 / 10)$ aufzuweisen $(p=0,021)$.

- Signifikant häufiger erhielten die def. Diagnosen "Hernie“ $(p=0,049)$, "Abszess/Phlegmone" ( $p=0,001)$ \& "Cholecystholithiasis" $(p=0,009)$ eine OP-Empfehlung und "Akutes Abdomen" $(p=0,000)$, "Abszess/ Phlegmone“ $(p=0,018) \&$ "Cholecystholiasis" $(p=0,003)$ nicht unerwartet eine unmittelbare OP-Indikation.

\section{Schlussfolgerung: Diese Studie zur chir. Konsiltätigkeit dient}

i) der chir. Qual.-Sicherung bei der klin. Mitbetreuung von Patienten mit interdisz., hier chir. Versorgungsbedarf in der tägl. allg.-/visz.-chir. Praxis im Rahmen der klin. Versorgungsforschung (und)

\section{ii) dem Klinik-Marketing.}

Die Daten reflektieren anschaulich das tägl. Arbeitsprofil eines allg.- \& visz.-chir. OA bei hohem Erfassungsgrad der aufgeführten Auswertparameter. 
PS153

Evaluation der Umsetzung ärztlicher Versorgungsleitlinien und pflegerischer Expertenstandards für die multimodale demenzspezifische Patientenversorgung im ambulanten Setting: ein internationaler Vergleich zwischen Deutschland und der Schweiz

Angelika Feldmann ${ }^{1}$; Christian Apfelbacher ${ }^{2}$; Maria Eberlein-Gonska ${ }^{3}$

${ }^{1}$ TU Dresden, München; ${ }^{2}$ UR-Universität Regensburg Fakultät Medizin Regensburg; ${ }^{3}$ Universitätsklinikum Carl Gustav Carus TU Dresden, Dresden

Hintergrund und Zielsetzung: Es ist sowohl für die Akteure der ambulanten (allgemein-) medizinischen Versorgung als auch für die Pflege bei demenziellen Erkrankungen nicht ausreichend untersucht, inwiefern strukturierte Entscheidungshilfen im medizinischen und pflegerischen Alltag berücksichtigt werden. Ziel dieser Studie ist es, die Sichtweisen von Experten aus dem ärztlichen und pflegerischen Bereich in Bezug auf Versorgungsleitlinien und Expertenstandards zu explorieren.

Methoden: In Deutschland und der Schweiz wurden unter Verwendung eines Interviewleitfadens 20 Experteninterviews mit Ärzten und Pflegepersonal im Bereich der Demenzversorgung durchgeführt. Die Interviews wurden digital aufgezeichnet und verbatim trankribiert. Die Auswertung erfolgte inhaltsanalytisch. Nach der Codierung des Interviewmaterials wurden Kategorien und Hauptkategorien gebildet und eine Bewertung vorgenommen.

Ergebnisse und Beantwortung der zentralen Forschungsfragen - Erkenntnisgewinn: Es wurden 36 Kategorien gebildet, die wiederum in 6 Hauptkategorien zusammengefasst wurden: 1. Einstellung, 2. Implementierung, 3. Patientenorientierung, 4. Praxistauglichkeit, 5. Evidenzbasiertes Verfahren und 6. Strukturmerkmal. Insgesamt lassen sich drei Typisierungen der Akzeptanz von Leitlinien und Expertenstandards unterscheiden: 1 . Leitlinien und Expertenstandards sind hilfreich, 2. Leitlinien und Expertenstandards werden für das Krankheitsbild "Demenz" nicht benötigt sowie 3. Die Umsetzung von Leitlinien und Expertenstandards sollte gefördert werden. Sowohl Ärzte als auch das Pflegepersonalbeurteilen systematisch entwickelte Entscheidungshilfen für das angemessene ärztliche sowie pflegerische Vorgehen ambivalent und skeptisch. Die Grundlage für eine gemeinsame und informierte Entscheidungsfindung von Ärzten, professionell Pflegenden und Patienten findet somit noch nicht vollumfänglich statt (mangelnde Patientenpartizipation). Die Befragten waren bereit, sich mit dem Thema der evidenzbasierten und konsensusunterstützten strukturierten Entscheidungshilfen substantiell auseinanderzusetzen. Im Ländervergleich bleibt festzuhalten, dass die Experten aus Deutschland und der Schweiz sowohl im ärztlichen als auch im pflegerischen Bereich grundsätzlich ähnliche Sichtweisen hatten.

Diskussion und Ausblick: Eine wichtige Voraussetzung zur Akzeptanz der ärztlichen Leitlinien und pflegerischen Expertenstandards stellt die Anpassung an die jeweiligen Herausforderungen und Bedingungen des Versorgungsalltags im ambulanten Setting dar. Das Forschungsvorhaben hat aufgezeigt, dass die ärztlichen Leitlinien zu demenziellen Erkrankungen im ambulanten Setting konkretere, an den spezifischen Belangen des multimorbiden und polymedizierten älteren Patienten ausgerichtet werden sollten. In diesem Zusammenhang wird auf die grundsätzliche Frage der Gewichtung einzelner Prozessstandards und Qualitätskriterien sowie die Möglichkeit der Unterteilung in Mindest- und Maximalstandards hingewiesen.
PS154

Magenkarzinom(Ca)-Übersichtsdaten im täglichen chirurgischen Management einer Studiengruppe aus einer prospektiven Multicenter-Beobachtungsstudie i.R. der klinischen Versorgungsforschung

Ingo Gastinger'; Frank Meyer²; . Studiengruppe „Primäres Magenkarzinom ${ }^{\prime \prime}$

${ }^{1}$ Otto-von-Guericke-Universität Magdeburg, Magdeburg; ${ }^{2}$ Universitätsklinikum Magdeburg A.ö.R., Magdeburg

Ziel \& Methode: Untersuchung d. bedeutsamsten Kriterien \& Faktoren (wie Fortschritte in diagnost. Prozeduren \& op. Techniken) mit Einfluss auf i) peri- \& früh-postop. „Outcome" sowie ii) onkolog. Langzeitergebnisse an konsekutiven Patienten mit histolog. gesichertem Magen-Ca, die in deutschen chir. Kliniken von I-XII/2002 sowie 20072009 (Einführung multimodaler Ther--Konzepte) im Rahmen einer prospektiven Multicenter-Beob.-Studie registriert wurden \& damit den chir. Alltag in der landesweiten Versorgung $d$. Magen-Ca reflektieren.

Ergebnisse (Eckpunkte): - Insgesamt ist die neoadj. Behandlung bis auf $18 \%$ angestiegen.

- Es gibt eine Verbesserung der 5-Jahres-Gesamtüberlebensrate (alle Stadien: von 40,0 auf 48,5\%, insbes. in Stadium II/IV) ohne erhöhtes periop. Risiko (kein Anstieg von postop. Morbidität/Letalität) nach Inauguration multimodaler Konzepte \& auf einer höheren D2-Lymphadenektomie-Rate basierend (von 71 auf $83,3 \%$ ) über die Jahre.

- Es war eine erhöhte Akzeptanz (jedoch mit weiterem Potenzial) der EUS-Nutzung (27,4 auf 40,4\%) zu verzeichnen, insbesondere um die Stadien "T>2" und "N+" zu eruieren, die für die neoadj. Behandlung relevant sind als auch eine „Über-/Unterbehandlung" zu vermeiden.

- Dysphagie \& Magenstenose (als Charakteristika für eine gestörte Gl-Passage durch Tu-induzierte Obstruktion als auch fortgeschr. Tu-Wachstum \& prähospitalen katabolen Status), Adipositas \& periop. Risiko nach ASA-Klassifikation wurden als unabh. Einflussfaktoren auf die Heilung der esophagojej. Anastomose mittels logistischer Regression eruiert.

- Ther.-Ergebnisse \& die Prognose der AEG-Tumore sind signifikant schlechter als Magen-Ca anderer Lokalisationen, was nicht befriedigen kann trotz multimodalen Herangehens \& Erhöhung der Resektionsrate als auch moderater Verbesserung von Morbidität, Letalität \& 5-Jahres-Überleben (jedoch auch Trend einer ansteigenden Anast.-Insuffizienzrate) über die Jahre.

- "Hospital-volume“-Effekte können lediglich in der Behandlung der prox. Magen-Ca (AEG-Tumorläsionen) nachgewiesen werden.

Es war eine verminderte Rate an Palliativeingriffen zu verzeichnen ( $40 \%$ auf $24,5 \%$ ). Die radikale Tu-Resektion unter palliativer Intention (wenn möglich aus Op-technischer Sicht) mündete in einer Verlängerung der medianen Überlebenszeit von 3 Monaten mit akzeptabler postop. Morbidität \& Letalität im Vergleich mit nichtresektiven Prozeduren.

- Patienten, die einer palliativen Op unterzogen wurden, ziehen Nutzen aus der postop. palliativen Chemotherapie, wobei, wie erwartet, die mit resezierenden Op's mehr als jene mit nichtresezierenden Verfahren.

Schlussfolgerung: Um das früh-postop. \& onkolog. Langzeitoutcome weiter zu verbessern, erscheinen insbesondere i) eine größerer 
Anteil neoadj. Behandlung \& laparoskop. Chirurgie beim Magen-Ca, ii) die Zentralisierung des prox. Magen-Ca \& iii) die palliative Resektion mit niedrigem Risiko (ASA, Ausschluss Tu-Stenose/Dysphagie) empfehlenswert.

\section{PS155 \\ Biologika zur Behandlung von Patienten mit chronisch-ent- zündlichen Darmerkrankungen (CED): Therapiekontinuität und assoziierte Arzneimittelkosten}

Antje Groth'; Thomas Wilke ; Alina Brandes ${ }^{3}$; Boris Ratsch ${ }^{3}$; Andreas Fuchs ${ }^{4}$; Barthold Deiters ${ }^{5}$; Bernd Bokemeyer ${ }^{6}$

'IPAM e.V., Wismar; ${ }^{2}$ Ingress Health HWM GmbH, Wismar; ${ }^{3}$ Takeda Pharma Vertrieb GmbH \& Co. KG, Berlin; ${ }^{4} \mathrm{AOK}$ PLUS, Dresden; ${ }^{5} \mathrm{GWQ}$ Serviceplus AG, Düsseldorf; ${ }^{6} \mathrm{Gastroenterologische} \mathrm{Gemeinschaftspraxis} \mathrm{Minden,} \mathrm{Minden}$

Hintergrund: Biologika spielen eine große Rolle bei der CED-Behandlung in Deutschland. Nachweise für Kontinuität und Wechselraten von initiierten Therapien sind kaum vorhanden, insbesondere für Patienten ohne vorherige Biologika-Behandlung (bio-naiv). Zudem haben Biologika einen erheblichen finanziellen Einfluss auf das Gesundheitssystem.

Ziel: Ziel dieser Interimsanalyse einer retrospektiven, deutschen Krankenkassen-Studie (7,5 Mio. Versicherte) war die Beschreibung von Wechsel- und Abbruchraten der initiierten Biologika-Therapien (anti-TNFs: Adalimumab, Golimumab, Infliximab; Vedolizumab (VDZ)) bei CED-Patienten und der damit verbundenen Arzneimittelkosten. Die Datenbasis beinhaltet stationäre und ambulante Behandlung, sowie erstattungsfähige Arzneimittelverordnungen.

Methoden: Erwachsene Patienten mit gesicherter Morbus Crohnoder Colitis Ulcerosa-Diagnose $(63 \% / 37 \%)$, die mit einer anti-TNFoder VDZ-Therapie zwischen 01.07.2015 - 30.06.2016 begannen und 12 Monate beobachtbar waren (oder bis zum Tod), wurden eingeschlossen. Die Beobachtung begann mit der ersten Biologika-Verordnung. Therapieabbruch und -wechsel wurden zwischen anti-TNF- und VDZ-Patienten unadjustiert und adjustiert nach Geschlecht und Alter verglichen (Log-Rank-Test und Cox-Regression). Therapiewechsel wurde definiert, als Verordnung eines anderen Biologikums und Therapieabbruch als Behandlungslücke von $>90$ Tagen. Die Arzneimittelkosten aus dem Apothekenabgabepreis wurden für Perioden von kontinuierlicher Index-Therapie in $€$ pro beobachtetem Patientenmonat berichtet.

Ergebnisse: 1.149 von 51.222 CED-Patienten begannen eine anti-TNFoder VDZ-Therapie im Einschlusszeitraum. Davon konnten für diese Analyse 582 Patienten für 12 Monate oder bis zum Tod beobachtet werden (476 anti-TNF: durchschnittlich 37,2 Jahre, 53,9\% weiblich, 78,7\% bio-naiv; 107 VDZ: durchschnittlich 40,5 Jahre, 55,1\% weiblich, $21,5 \%$ bio- naiv). Im Follow-up wechselten $20,6 \%$ vs. $16,8 \%$ der anti-TNF- vs. VDZ-Patienten $(\mathrm{p}=0.227) \mathrm{zu}$ anderen Biologika (bio-naiv: $17,9 \%$ vs. $17,4 \%, p=0.605) ; 19,8 \%$ vs. $15,0 \%(p=0.154)$ brachen ihre Therapie ab (bio-naiv: $20,6 \%$ vs. $21,7 \%, p=0.534$ ) und $59,4 \%$ vs. $67,6 \%$ setzten ihre Therapie fort ( $N=4$ zensiert; $\mathrm{p}=0.060$; bio-naiv: $61,5 \%$ vs. $60,9 \%)$. Patienten verblieben im Durchschnitt länger auf VDZ (309 Tage) als auf anti-TNF (286 Tage; $p=0.028$; bio-naiv: 309 vs. 293 Tage). Durchschnittliche Arzneimittelkosten pro Patientenmonat in der Zeit auf Therapie betrugen $2.561,66 €$ für anti-TNF- und 2.314,74€ für VDZ-Therapie.

Schlussfolgerungen: Dies ist die erste Analyse eines großen Datensatzes zu Therapiekontinuität und Kosten von Biologika in Deutschland. Im Vergleich zu bisherigen observationalen Studien, ermittelte diese Studie niedrigere Persistenz-Raten von etwa zwei Dritteln der
CED-Patienten nach 12 Monaten. Es gibt eine Tendenz zu längerer Therapiekontinuität bei Patienten, die mit VDZ behandelt wurden, bei vergleichbaren Arzneimittelkosten sowohl in der Gesamt- als auch in der bio-naiven Population.

\section{PS156 \\ Informationsübermittlung von stationär nach ambulant bei Patienten mit akuter Nierenschädigung}

Anja Haase-Fielitz'; Bernt-Peter Robra ${ }^{2}$; Anke Spura ${ }^{3}$; Christian Albert ${ }^{4}$; Christian Butter'; Martin Ernst ${ }^{5}$; Michael Haase ${ }^{6}$

${ }^{1}$ Herzzentrum Brandenburg, Bernau; ${ }^{2}$ Institut für Sozialmedizin und Gesundheitsökonomme, Magdeburg; ${ }^{3}$ Instut für Sozialmedizin und Gesundheitsökonomie, Magdeburg; ${ }^{4}$ Diaverum MVZ Potsdam, Potsdam; ${ }^{5}$ Medizinische Fakultät, Otto-von-Guericke-Universität Magdeburg, Magdeburg; ${ }^{6}$ Diaverum MVZ Potsdam \& Medizinische Fakultät, Otto-von-Guericke-Universität Magdeburg, Potsdam

Hintergrund: Die akute Nierenschädigung (AKI) kann zur Progredienz einer vorbestehenden chronischen Niereninsuffizienz (CKD) bis hin zur Dialysepflichtigkeit beitragen. Der Nutzen (Letalitätssenkung) der fachspezifischen ambulanten Nachsorge nach stattgehabter akuter Nierenschädigung ist belegt. Die primäre Fragestellung der vorliegenden Untersuchung war es, Quantität und Qualität der Informationsübermittlung über "AKI-bei-CKD" stationär versorgter Patienten an ambulant nachsorgende Ärzte zu untersuchen.

Methodik: Der Entlassungsarztbrief von 150 konsekutiv an einem Universitätsklinikum behandelte Patienten mit vorbestehender CKD, welche während ihrer stationären Behandlung eine AKI aufwiesen, wurde untersucht. Ausgeschlossen wurden Patienten mit CKD Stadium 5 bzw. chron. Dialysepflichtigkeit und auf der nephrologischen Station behandelte Patienten.

Ergebnisse: Das Alter der Patienten lag bei 72 Jahren (25.-75. Perzentile 63-78), der Frauenanteil bei 41\%. Die Nierenfunktion war zum Zeitpunkt der stationären Aufnahme deutlich eingeschränkt (geschätzte glomeruläre Filtrationsrate $49 \mathrm{ml} / \mathrm{min}$ [25.-75. Perzentile 31-70]). AKI Grad 1 wiesen 60\% der Patienten auf, Grad $218 \%$ und Grad $322 \%$. Die Krankenhausletalität betrug $20 \%$ und die Wiederaufnahmerate ins Krankenhaus innerhalb von 30 Tagen $18 \%$. Bei $28 \%$ der betroffenen Patienten wurde die Diagnose ,AKI' in der Diagnoseliste des Entlassbriefes geführt und bei $30 \%$ auf eine während des stationären Aufenthalts akut abfallende Nierenfunktion hingewiesen. In 9\% aller Fälle wurde die Empfehlung zur ambulanten Kontrolle der Nierenfunktion und in 5\% der Fälle zur fachspezifischen ambulanten Nachsorge gegeben, wobei letztere Empfehlung etwas häufiger gegeben wurde (18\%), wenn bei diesen Patienten während der stationären Behandlung ein nephrologisches Konsil durchgeführt worden war. Bei $19 \%$ der Patienten lag die niedrigste Nierenfunktion zum Zeitpunkt der Entlassung aus dem Krankenhaus vor. Bei 15\% der Patienten wurden im Entlassbrief Angaben zum Schweregrad, Ursachen und Therapie der AKI gemacht. Die Diagnose ,AKI' wurde bei $27 \%$ der Patienten in der stationären Patientenakte dokumentiert. Die Kodierung der Diagnose , $\mathrm{AKI}^{\prime}$ erfolgte bei $25 \%$ der Patienten.

Limitation: Die Studie kann den tatsächlichen Umfang und die Art und Qualität der erfolgten Nachsorge sowie ihr Ergebnis nicht beurteilen.

Diskussion: Im beschriebenen Risikopatientenkollektiv wurde die seltene Weitergabe der Diagnose ,akute Nierenschädigung' an ambulant nachsorgende Ärzte belegt. Bei einem erheblichen Anteil der Patienten lag die schlechteste Nierenfunktion zum Zeitpunkt der Entlassung aus dem Krankenhaus vor. Eine Empfehlung zur fachspezifi- 
schen Weiterbehandlung wurde nur in Ausnahmefällen gegeben. Die Ergebnisse der vorliegenden Arbeit entsprechen der internationalen Datenlage, wobei außerhalb Deutschlands bereits Versorgungsforschungsprogramme zur Kontextanalyse und Optimierung der intersektoralen Schnittstelle angelaufen sind.

\section{PS157 \\ Blood pressure, lipid lowering and self-reported adherence with a fixed-dose combinaton of Atorvastatin/Perindopril/Amlodipi- ne in primary care: a non-interventional study}

Stephan Jacob'; Bettina Weger ${ }^{2}$

${ }^{1}$ Universität Tübingen, Villingen-Schwenningen; ${ }^{2}$ Servier Deutschland $\mathrm{GmbH}$, München

Objective: To investigate the effect on blood pressure (BP), lipid parameters (LP) and adherence to medication after 4 months of therapy with a fixed-dose combination (FDC) of Atorvastatin/Amlodipine/Perindopril (ATOR/PER/AMLO) in hypertensive patients with concomitant hypercholesterolemia or mixed hyperlipidaemia.

Design and Method: We performed a prospective, non-randomized study on the effect of 4 months of therapy with FDC ATOR/PER/ AMLO medication in patients with hypertension and concomitant hypercholesterolemia or mixed hyperlipidaemia, who were previously treated with single pill formula. Changes in BP were assessed by office $\mathrm{BP}$ and home BP. Lipid parameters were recorded as part of the medical routine. Adherence was measured with the Hill-Bone medication adherence scale.

Results: 1134 patients (39.5\% women) were enrolled, mean age was 65.7 years, $38.6 \%$ were 70 years or older, mean body mass index was $29.7 \mathrm{~kg} / \mathrm{m} 2$. 1017 patients completed the 4 months follow-up, $41.9 \%$ suffered from Hypertension longer than 10 years and hypercholesterolemia was diagnosed in $93.7 \%$ for more than one year (851patients). $74.5 \%$ had other risk factors (e.g. obesity $39.9 \%$, smoking $30.7 \%$, pre-diabetes $15.2 \%$ of all patients) and $85.4 \%$ had concomitant diseases (e.g. diabetes mellitus 22.4\%, COPD 10.0\%, CVI 8.1\%, CHD $6.4 \%$ of all patients)

Majority of patients were pretreated with statins (82.79\%), $78.4 \%$ with ACE inhibitors and $58.5 \%$ with calcium channel blockers in a single pill strategy. Main reason to start therapy with ATOR/PER/AMLO was the reduction of the pill burden ( $84.2 \%)$ and non-adherence to pre-medication (37.4\%).

$83.4 \%$ of patients had concomitant therapies. The most prevalent co-medications were betablocker (50.7\%), Antiplatelet drugs $(26.0 \%)$ and oral hypoglycaemic agents (17.2\%).

$90.4 \%$ of patients did not have to change the dose of ATOR/PER/AMLO during the course of the study. The ATOR/PER/AMLO dose schedule at start was quite divers, but most often patients received 20mg/10mg/5mg ATOR/PER/AMLO (28.0\%).

After 4 months, office BP decreased from 154.5/90.0 $\mathrm{mmHg}$ at inclusion to $133.4 / 79.0 \mathrm{mmHg}$ at the last documentation. The responder rate was $69.6(\mathrm{BP}<140 / 90 \mathrm{mmHg})$.

Total Cholesterol levels improved statistically significant by - 44.5 $( \pm 44.3) \mathrm{mg} / \mathrm{dl}$ from visit $1-3(p<0.0001)$ with a mean final total Cholesterol of $187.1 \mathrm{mg} / \mathrm{dl}$. HDL level increased by $3.4( \pm 12.5) \mathrm{mg} / \mathrm{dl}(\mathrm{p}<0.0001)$ with a mean final HDL of $53.5 \mathrm{mg} / \mathrm{dl}$. LDL level were reduced by 35.06 $( \pm 38.2) \mathrm{mg} / \mathrm{dl}(\mathrm{p}<0.0001)$ with a mean final LDL of $108.5 \mathrm{mg} / \mathrm{dl}$.
Perfect adherence at 4 months markedly increased from 164 patients $(15.9 \%)$ at baseline to 365 patients $(35.3 \%)$ at the end of the study.

Conclusion: ATOR/PER/AMLO as FDC can be successfully used in patients with hypertension with concomitant hypercholesterolemia or mixed hyperlipidaemia and shows significant improvement in adherence. At the end, a much better blood pressure and lipid control was achieved possibly due to a better adherence.

\section{PS158}

Die Nutzung einer AND ( Allow Natural Death) Anordnung in der täglichen Praxis. Die Erfahrung am Klinikum Bremen Mitte (KBM)

Antonia Karpouza'; Klaus-Peter Hermes ${ }^{2}$; Brigitte Kuss²; Gisela Exeriede ${ }^{3}$; Jörg Gröticke ${ }^{2}$; Christoph Reuter ${ }^{4}$; Bernd Hertenstein ${ }^{2}$

${ }^{1}$ Helios Klinik Cuxhaven, Cuxhaven; ${ }^{2}$ Klinikum Bremen Mitte, Bremen; ${ }^{2} \mathrm{Kli}-$ nikum Bremen Mitte, Bremen; ${ }^{3}$ Helios Klinik Medizincontrolling, Cuxhaven; ${ }^{4}$ Medizinische Hochschule Hannover, Hannover

Einleitung: Die moderne Medizin bietet zunehmende Möglichkeiten zur Lebensverlängerung an. Mit der Frage der Sinnhaftigkeit einer Therapie setzen sich Ärzte und Pflegekräfte täglich auseinander. In vielen Kliniken sind Maßnahmen zur Anordnung von Therapiebegrenzungen (TBG) eingeführt, systematische Auswertungen zum Einsatz in der Praxis sind jedoch nur vereinzelt verfügbar.

Ziel: Im Klinikum Bremen Mitte wurde 2013 unter dem Begriff AND (Allow Natural Death) eine Anordnung zur schriftlichen Fixierung einer TBG eingeführt. In der AND Anordnung wird Art und Grund der TBG beschrieben und zwei Ärzte und eine Pflegekraft sollen die Anordnung unterschreiben. Das Ziel der aktuellen Arbeit ist es den Einsatz des AND Bogens auszuwerten.

Patienten und Methode: Die digitalen Akten von in 2015 und 2016 im KBM verstorbenen Pat. wurden retrospektiv recherchiert. Alle Daten bzgl. des Vorliegens einer AND Anordnung und deren Inhalt wurden gesammelt und analysiert.

Ergebnisse: Die Ergebnisse werden in der endgültigen Präsentation aktualisiert. Bislang wurden 633 verst. Pat. (58\% Männer, medianes Alter $76,4 \mathrm{~J}$ ) analysiert. Bei 455 (72\%) Pat. wurde eine AND Anordnung getroffen. Der Anteil von Pat. mit AND Anordnung ist am höchsten bei Pat. aus hämatoonkologischen (84/94,89\%), gastroenterologischen $(65 / 71,92 \%)$ und neurologischen Stationen $(56 / 64,88 \%)$.

Der Anteil von Pat. mit AND Anordnung unterschied sich je nach vorliegender Diagnose. Am höchsten war er bei malignen Krankheiten (148/177,84\%), am geringsten bei Trauma (z.B. Verkehrsunfall) (10/24, $42 \%)$. Sie waren ebenfalls häufig bei resp. Insuffizienz (44/55, 80\%) und Apoplex (98/126,78\%) und deutlich niedriger bei kardiovaskulären Krankheiten (54/85, 64\%), Leberzirrhose $(15 / 23,65 \%)$ oder Sepsis (41/65,63\%).

Als Grund für die AND Anordnung wurde in 70\% der Fälle eine fehlende medizinische Indikation zur Therapieeskalation angegeben, in $10 \%$ der Wunsch des Pat., in $12 \%$ der mutmaßliche Wille des Pat. und in $6 \%$ eine vorliegende Patientenverfügung. In 5 Fällen war der Grund nicht dokumentiert. Die mediane Aufenthaltsdauer war 6 Tage(T) (o155), die Zeit zwischen Aufnahme und AND $2 T$ (o-93), zwischen AND und Tod $3 \mathrm{~T}(0-82)$. Die AND Anordnung wurde generell berücksichtigt. Ausnahme war ein Pat., der trotz anderslautender AND Anordnung auf die ITS verlegt wurde. Bemerkenswert ist die Tatsache, dass lediglich in 12\% der Fälle neben einem Arzt eine Pflegekraft und nur in $4 \% 2$ Ärzte und eine Pflegekraft die AND Anordnung unterschrieben. 
Diskussion: Nach unseren Daten wird die AND Anordnung in hohem Maße im klinischen Alltag des Klinikums benutzt um eine TBG Entscheidung nachvollziehbar dem behandelten Team zu kommunizieren. Die AND Anordnung ist für Ärzte klar und wird in der Therapie berücksichtigt. Die Wahrscheinlichkeit einer AND Anordnung liegt bei malignen oder neurologischen Erkrankungen deutlich höher als beispielwiese bei kardiovaskulären Erkrankungen, auch wenn häufig ähnliche Prognosen vorliegen.

\section{PS159 \\ Zeigt ein 5-Jahres-Zeitraum Trends bei der chirurgisch-operati- ven Versorgung des Rektumkarzinoms an?}

Julia Katzenstein ; Frank Meyer ${ }^{2}$; Ingo Gastinger ${ }^{3}$; Henry Ptok ${ }^{2}$; Hans Lippert $^{3}$; Ralf Steinert ${ }^{4}$

'AMEOS Klinikum Aschersleben, Aschersleben; ${ }^{2}$ Universitätsklinikum Magdeburg AöR, Magdeburg; ${ }^{3}$ An-Institut für Qualitätssicherung in der operativen Medizin Magdeburg, Magdeburg; ${ }^{4}$ St. Josefs-Krankenhaus Salzkotten, Salzkotten

Hintergrund: Gendermedizinische Aspekte spielen eine zunehmende Rolle in der klinischen Medizin und Onkochirurgie.

Ziel \& Methode: Mittels klinisch-systematischer, prospektiver multizentrischer Beobachtungsstudie sollten die Trends bezüglich Änderungen des Gesamtoutcomes im zeitlichen Verlauf an einer repräsentativen Fallzahl zur chirurgischen Therapie des Rektumkarzinoms über zwei definierte Zeitraume inclusive geschlechtsspezifischer Unterschiede zu analysieren.

Ergebnisse: Patientenzahl: 10.657 (2005/06: $\mathrm{n}=5.714 ;$ 2010/11: $\mathrm{n}=4.943)$ in der Mehrheit männlich (60,9\%). Exog./endog. Risikofaktoren: Männer unterliegen signifikant häufiger(gleich in beiden Zeiträumen) dem Alkohol-/Nikotinmissbrauch. Anteil adipöser Patienten ist bei beiden Geschlechtern zunehmend. Rate anderer exog. Risikofaktoren bleibt über die Jahrgänge annähernd stabil.

Die MRT- \& EUS-Untersuchungszahl steigt über die Jahrgänge an (jeweils $\mathrm{P}$

\section{PS160}

\section{Klug entscheiden beim geriatrischen multimorbiden Patienten}

Annette Kurrle; Anja Hergenröther

Asklepios Paulinen Klinik Medizinische Klinik II, Wiesbaden

Anamese: 87-jähriger Patient wurde zur geriatrischen Komplexbehandlung übernommen nach Erstdiagnose eines Bronchial-CA (großzellig). Weiterhin erwähnenswert bekannte COPD, stabile Koronare Herzkrankheit, arterieller Hypertonie, Chronisches Schmerzsyndrom bei degenerativen Veränderungen.Körperlicher Status: Patient in leicht reduziertem AZ und regelrechtem EZ $(173 \mathrm{~cm}, 72 \mathrm{~kg}$, BMI 24), HF:98'min, Spo2:89\%, AF: 19'min. Cor:Herztöne leise, Aktion rhythmisch mit vereinzelten ES. Pulmo:hypersonorer Klopfschall, leises vesikuläres AG, beidseits basal Brummen. Kopf- und Halsstatus: cervikal Lymphknotenvergrößerungen palpabel. Regelrechter Abdominalstatus. Wirbelsäule:diffuser Klopfschmerz v.a. über BWS, Beweglichkeit schmerzhaft eingeschränkt. Gelenke: große Gelenke arthrotisch deformiert , keine Rötung, keine Überwärmung, keine Ergüsse. Neurologischer Status: regelrechtes Reflexniveau, Stand und Gangunsicherheit, Romberg, Unterberger nicht durchführbar. Psychiatrischer Befund: Patient erschöpft, keine wesentlichen kognitiven
Einschränkungen. Labor:CRP 15mg/dl, HB:12g/dl, Leukos 12000, weitere Laborparameter im Normbereich.

Therapie und Verlauf: Im Vordergrund der Therapiemassnahmen stand initial die allgemeine Stabilisierung, Verbesserung des Bewegungsvermögens. Onkologischerseits war eine operative Therapie abgelehnt worden. Von Seiten des Schmerzsyndromes war der Patient unter einer Kombination aus Novaminsulfon und Tilidin gut eingestellt. Unter einem moderaten Therapie- und Trainingsprogramm konnte zunächst eine deutliche Stabilisierung und Verbesserung der Mobilität erreicht werden. Nach 10 Tagen Verschlechterung des AZ mit $\mathrm{Hb}$-Abfall auf 7,3g/dl, Verschlechterung der pulmonalen Situation, Fieber. Radiologisch pneumonische Infiltrate beideits. Massiver Ganzkörperschmerz. Verlegung auf die Intermediate Care. Gabe von Erythrozytenkonzentraten, kalkulierte Breitbandantibiose, trotz leichter Stabilisierung lehnt der Patient weitere diagnostische Massnahmen, (Gastroskopie) ab. Mit dem Patienten und seinen Angehörigen wird ein palliatives Procedere besprochen. Nach Rückverlegung auf Normalstation, adäquater Schmerztherapie, Best supportive Care verstarb der multimorbide Patient nach einer Woche.

Zusammenfassung: Die Kasuistik zeigt in eindrücklicher Weise den wechselhaften klinischen Verlauf bei multimorbiden geriatrischen $\mathrm{Pa}$ tienten. Trotz Multimorbidität konnte eine kurze Phase der Stabilisierung erreicht werden. Das Eintreten von Komplikationen, für welche ein erhöhtes Risiko vorliegt, zieht nicht nur eine rasche Veränderung des Verlaufes nach sich, sondern bedingt auch eine Korrektur des Therapiezieles. Eine kluge Entscheidung zu treffen zwischen dem medizinisch Machbaren und dem ethisch Vertretbaren, unter gleichzeitiger Berücksichtigung der Patientenautonomie stellt insbesondere in der Geriatrie eine besondere Herausforderung an das therapeutische Team, den Patienten und seine Angehörigen.

\section{PS161}

Therapiemanagement des Reizdarmsyndroms in der hausärztlichen Praxis: Probiotika und Phytotherapeutika kommen häufig erfolgreich zum Einsatz

Ahmed Madisch'; Christian Labenz ${ }^{2}$; Margrit Hollenz ${ }^{3}$; Manfred Gross ${ }^{4}$; Joachim Labenz ${ }^{5}$

${ }^{1}$ Klinikum Region Hannover - Klinikum Siloah, Hannover; ${ }^{2}$ Universitätsmedizin Mainz, Mainz; ${ }^{3}$ Praxis für Allgemeinmedizin, Rödental; ${ }^{4}$ Internistische Klinik München Süd, München; ${ }^{5}$ Diakonie Klinikum GmbH • Jung-Stilling-Krankenhaus, Siegen

Einleitung: Das Reizdarmsyndrom (RDS) gehört in der primärärztlichen Versorgung zu den häufigsten funktionellen Störungen des Gastrointestinaltraktes. Bei noch unzureichend verstandener Pathophysiologie und fehlendem kausalen Therapieansatz ist die Therapie nach wie vor eine Herausforderung in der täglichen Praxis.

Patienten und Methodik: Im Rahmen einer deutschlandweiten Fragebogenaktion wurden in der Primärversorgung tätige Ärzte (Allgemeinmediziner und Internisten) postalisch angeschrieben und gebeten, einen Fragebogen auszufüllen. Dabei lag der Schwerpunkt des Fragebogens auf dem therapeutischen Management von RDS-Patienten und den persönlichen Erfahrungen mit den verschiedenen medikamentösen Therapieoptionen.

Ergebnis: Insgesamt beteiligten sich 478 Ärzte aus allen Bundesländern an dieser Umfrage. Mehr als $50 \%$ der Patienten mit RDS präsentieren sich mit multiplen Symptomen und berichten dabei über eine starke Einschränkung der Lebensqualität (70,3\%). Nach Einschätzung der Ärzte spielen Stress (76\%), das Darmmikrobiom (56\%), die falsche 
Ernährung (35\%) sowie die Sensitivitätsstörung (26\%) die wesentliche Rolle bei der Entstehung des RDS. Die am häufigsten eingesetzten Medikamente, auch nach Empfehlung vom Facharzt, sind Spasmolytika (43,5\%), Phytotherapeutika (37,7\%) und Probiotika (37,8\%). Die Zufriedenheit mit dem Therapieerfolg bei Einsatz dieser Substanzen lag bei 53,8\% (Spasmolytika), 74,7\% (Phytotherapeutika) und $74.9 \%$ (Probiotika). Beim Einsatz von trizyklischen Antidepressiva sind mehr als $60 \%$ der Ärzte unzufrieden. Die hohe Beratungsintensität $(71,8 \%)$ und Erwartungshaltung $(73,4 \%)$ sowie die wechselnde Symptomatik $(44,9 \%)$ der Patienten werden von den Ärzten als größte Herausforderungen in der täglichen Praxis angegeben.

Schlussfolgerungen: Das Reizdarmsyndrom gehört zu den häufigen Erkrankungen in der primärärztlichen Versorgung. Dabei stellen die Polysymptomatik, die hohe Beratungsintensität und Erwartungshaltung durch die Patienten die größten Herausforderungen dar. Probiotika und Phytotherapeutika kommen häufig erfolgreich zum Einsatz.

\section{PS162 \\ The influence of diabetes mellitus onto "morbidity and mortali- ty" and survival rates after rectal cancer resections}

Frank Meyer'; Björn Gebauer'; Henry Ptok'; Ralf Steinert ${ }^{2}$; Ronny Otto ${ }^{3}$; Ingo Gastinger ${ }^{3}$; Hans Lippert ${ }^{3}$

${ }^{1}$ Universitätsklinikum Magdeburg A.ö.R., Magdeburg; ${ }^{2}$ St Joseph Hospital, Salzkotten; ${ }^{3}$ Otto-von-Guericke-Universität Magdeburg, Magdeburg;

${ }^{3}$ Otto-von-Guericke-Universität Magdeburg, Magdeburg

Introduction: Diabetes mellitus (DM) is a common comorbidity. Especially in rectal cancer surgery with older patients, the prevalence is about one in six patients having DM. This makes it crucial as aim of the study to examine the possible impact of DM onto important early postoperative \& long-term oncological outcomes in this type of surgery.

Methods: A prospective multicenter observational study was conducted from 2008 to 2011 observing 68 items, concerning pre-, peri-, postoperative (postop.) aspects $\&$ histological findings. These were reported by the clinic where the patient underwent surgery. A consecutive follow-up was performed if the patient signed a consent form. Patients were grouped as non-diabetic (w/o DM) \& not-/insulin-dependent DM (NIDDM/IDDM). Further discrimination was made in morbidity \& mortality analyses, dividing into patients younger than 65 years (yr), aged 65 to $74 \mathrm{yr} \& 75 \mathrm{yr}$ as well as above.

Results: In total, 10,442 patients were enrolled including 8,546 (81.8\%) patients w/o DM, 1,145 (11.0\%) w/ NIDDM \& 751 (7.2\%) w/ IDDM. Mean age at admission was 68 (range, 21-99) yr with older patients in the IDDM \& NIDDM group $(P<0.001)$. Tumor stages (according to UICC classification) were comparable $(P=0.547)$. ASA score was worse in IDDM \& NIDDM $(P<0.001)$. IDDM \& NIDDM increased postoperative morbidity (mean, 38.3\%) only in patients $65-74 \mathrm{yr}$ of age $(P=0.006)$. In-hospital mortality (mean, 3.2\%) was only impaired in patients younger than $65 \mathrm{yr}(P=0.011)$. The overall survival after $5 \mathrm{yr}$ was $60.6 \%$ in patients w/o DM whereas NIDDM (53.3\%) \& IDDM (46.4\%) patients had a worse outcome $(P<0.001)$. There was also a significant difference between NIDDM \& IDDM $(P=0.008)$. The disease-free survival (DFS) after $5 \mathrm{yr}$ was observed to be $58.5 \%$ in patients w/o DM. NDDIM \& IDDM patients had a worse DFS $(P<0.01)$ w/o differences between the DM groups $(P=0.106)$. A trend was observed that NIDDM-patients had a higher local recurrence rate after $5 \mathrm{yr}$, which was $7.8 \%$ compared to $8.3 \%$ in IDDM \& $4.6 \%$ in patients $\mathrm{W} / \mathrm{o}$ DM $(P=0.058)$.

Conclusions: DM worsened postop. morbidity \& mortality, although these impacts were only significant in certain age groups. Furthermo- re, survival rates were lower in DM patients, which might be at least partly due to higher age at admission \& worse preoperative physical status (characterized by ASA score). IDDM impaired the survival even more than NIDDM whereas NIDDM had a negative impact onto local tumor recurrence.

\section{Versorgungsstrukturen - Versorgungsforschung II}

\section{PS164}

Transsektorales Interventionsprogramm zur Verbesserung der Geriatrischen Versorgung in Regensburg [TIGER] Ein innovatives Versorgungsformen-Projekt (Innovationsfonds des G- BA)

Benjamin Schleenvoigt ${ }^{1}$; Markus Sellmann ${ }^{2}$; Christian Schulz ${ }^{3}$; Michael Waizmann ${ }^{4}$; Carlos Fritzsche ${ }^{5}$; Ines Ruck ${ }^{6}$; Nils Postel7; Hans Jäger ${ }^{8}$; Steve Rößler'; Ute Boashie ${ }^{10}$; Andreas Jenke ${ }^{11}$; Sophie Schneitler ${ }^{12}$; Thomas Heuchel $^{13}$; Mathias W. R. Pletz ${ }^{14}$

${ }^{1}$ Uniklinikum Jena; ${ }^{2}$ Medical practice, Adele-Sandrock-Straße $5 ;{ }^{3}$ University Hospital LMU Munich; ${ }^{4}$ Medical practice, Delitzscher Str. 137, Leipzig; ${ }^{5}$ University of Rostock Medical School; ${ }^{6}$ Medical practice, Käthe-Kollwitz-Str. 9, Leipzig; ${ }^{7}$ Medical practice, Sendlinger-Tor-Platz 8, Munich; ${ }^{8}$ HIV Research and Clinical Care Centre; ${ }^{9}$ Medical practice, Bürgerstrase $2 ;{ }^{10}$ Medical practice, Kamelienweg 2; ${ }^{11}$ Medical practice, Konigsbrücker Str. 76; ${ }^{12}$ Bethanien Hospital, Solingen; ${ }^{13}$ Medical practice, Carolastraße 1; ${ }^{14}$ Jena University Hospital

Einleitung: Der demographische Wandel wird in den kommenden Jahren zu einer zunehmenden Zahl an Krankenhausfällen durch ältere, chronisch erkrankte Menschen führen. Gerade bei älteren Patienten fördert ein Krankenhausaufenthalt beträchtliche Funktionseinschränkungen, Selbststän-digkeits- und Lebensqualitätsverluste, Ernährungsdefizite und erhöhte Infektionsrisiken, die nach Entlassung zu gravierenden Versorgungslücken führen können. Damit steigt das Risiko von Wie-dereinweisungen. Die Notwendigkeit einer hier angreifenden multiprofessionellen, integrierten Versorgung für ältere, vulnerable Patienten wurde in Deutschland erkannt und daher seitens des Gesetzgebers seit 2012 das Entlassmanagement als Bestandteil der Krankenhausbehandlung einge-führt. Praktische Erfahrungen zeigen jedoch, dass das Entlassmanagement den komplexen Bedürf-nissen der geriatrischen Patienten und ihrer Angehörigen oft nicht genügt, da es häufig nicht über eine Klärung von Leistungsansprüchen und Kontaktherstellung zu ambulanten Diensten hinausgeht. International begegnet man dem Defizit der patientengerechten Versorgung bei der Transition von stationärer zu ambulanter Behandlung u.a. mit Anwendung des Transitional Care Models (TCM), das von Mary Naylor et al.* entwickelt wurde, um älteren Patienten über eine Reihe definierter Aktionen eine Unterbrechung der Versorgungskette zu ersparen. Machbarkeit und Effizienz des Modells bezüglich eines verbesserten Gesundheitszustandes und einer Reduzierung der Kranken-hauswiedereinweisungsraten und Gesundheitskosten, sind durch Studien belegt.

Ziel: Ziel des sektorenübergreifenden Versorgungsformenprojektes TIGER ist die Reduzierung der Wiedereinweisungsrate. Dies soll durch eine Verbesserung der in Deutschland noch unzureichen-den Versorgung geriatrischer Patienten beim Übertritt von stationärer zu ambulanter Versorgung erreicht werden.

Methode: Das TIGER Programm bindet die Komponenten des wissenschaftlichen TCM in einem Projekt mit dem Krankenhaus Barmherzige Brüder in Regensburg ein. Eine strukturierte, kontinu-ierliche Begleitung geriatrischer Patienten, ihrer Angehörigen, ihres Arztes und Pflegeteams durch einen sog. "Pfadfinder" bereits während eines Krankenhausaufenthaltes und über weitere 12 Mo-nate danach unterstützt hierbei Patienten und pflegende Angehörige, eigenver- 
antwortlich und selbstbestimmt eine Stabilisierung bzw. Verbesserung von Lebensqualität, Krankheitsbewältigung, Funktionalität, Ernährungszustand und Wundversorgung zu erreichen. Wirksamkeit, Praktikabilität und Limitationen werden wissenschaftlich evaluiert, sowie eine mögliche Kostenersparnis für das Gesundheitssystem ökonomisch evaluiert. Das Projekt startete im September 2017 und hat eine Laufzeit von 36 Monaten.

Ergebnis: Durch das Projekt wird eine integrative Verzahnung von stationären und ambulanten Versorgungsteams rund um den Patienten angestrebt, die das Entlassmanagement komplementär ergänzt.

* Naylor M. et al. Ann Intern Med, 1994, 120, 999-1006

\section{PS165}

\section{HIV-regional: regionale Unterschiede des HIV-Testverhaltens}

Benjamin Schleenvoigt ; Markus Sellmann ${ }^{2}$; Christian Schulz ${ }^{3}$; Michael Waizmann ${ }^{4}$; Carlos Fritzsche ${ }^{5}$; Ines Ruck ${ }^{6}$; Nils Postel'; Hans Jäger ; Steve Rößler'; Ute Boashie ${ }^{10}$; Andreas Jenke ${ }^{11}$; Sophie Schneitler ${ }^{12}$; Thomas Heuchel $^{13}$; Mathias W. R. Pletz ${ }^{14}$

${ }^{1}$ Uniklinikum Jena, Jena; ${ }^{2}$ Medical practice, Adele-Sandrock-Straße 5, Berlin; ${ }^{3}$ University Hospital LMU Munich, Munich; ${ }^{4}$ Medical practice, Delitzscher Str. 137, Leipzig, Leipzig; ' University of Rostock Medical School, Rostock; ${ }^{6}$ Medical practice, Käthe-Kollwitz-Str. 9, Leipzig, Leipzig; ${ }^{7}$ Medical practice, Sendlinger-Tor-Platz 8, Munich, Munich; ${ }^{8}$ HIV Research and Clinical Care Centre, Munich; ${ }^{9}$ Medical practice, Bürgerstraße 2, Chemnitz; ${ }^{10}$ Medical practice, Kamelienweg 2, Dresden; ${ }^{11}$ Medical practice, Königsbrücker Str. 76, Dresden; ${ }^{12}$ Bethanien Hospital, Solingen, Solingen; ${ }^{13}$ Medical practice, Carolastraße 1, Chemnitz; ${ }^{14}$ Jena University Hospital, Jena

Hintergrund: Nach Angaben des Robert-Koch-Instituts (RKI) betreffen $30 \%$ aller HIV-Erstdiagnosen in Deutschland "late presenter" mit weniger als $200 \mathrm{CD} 4$-Zellen/ $\mu$ l. Die späte Diagnose und der späte Therapiebeginn gehen mit einer schlechteren Prognose für die betroffenen Patienten einher.

Methode: In diesem Studienprojekt wurden 289 HIV-Erstdiagnosen aus dem Jahr 2014 im Hinblick auf testende Einrichtung (Klinik Hausarzt, ambulanter Facharzt, AIDS-Hilfe und Gesundheitsamt) und CD4-Höhe bei Diagnosestellung ausgewertet. Es wurden 239 Erstdiagnosen aus den ostdeutschen Bundesländern in die Auswertung eingeschlossen und mit 50 Erstdiagnosen aus Münchner HIV-Schwerpunktpraxen verglichen.

Ergebnisse: 89 (31\%) der Diagnosen wurden in der Klinik gestellt, gefolgt vom Gesundheitsamt mit 53 (18\%). In je 16\% erfolgte die Diagnosestellung durch den Hausarzt bzw. ambulanten Facharzt (jeweils $\mathrm{n}=46$ ). Nur 9 Diagnosen (3\%) erfolgten nach einem Test durch die AIDS-Hilfe (Rest: Gesundheitsamt $n=53$, Blutspende $n=8$, Asyl $n=17$, sonstige $n=8$, unbekannt $n=13)$. $C D 4$ lag bei im Krankenhaus getesteten Patienten durchschnittlich bei $340 / \mu \mathrm{l}$, während Patienten, die beim Hausarzt getestet wurden, höhere $\mathrm{CD}_{4}$-Werte bei Diagnosestel-

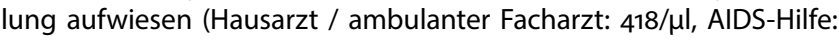

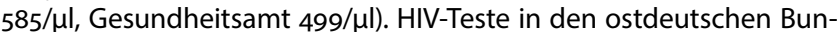
desländern waren mit im Mittel niedrigeren $\mathrm{CD}_{4}$-Zellen bei Diagnosestellung assoziiert, als Teste in der westdeutschen Großstadt (405 vs. 494/ $\mathrm{\mu l} ; \mathrm{p}=0,03)$. HIV-Teste, die im Krankenhaus veranlasst wurden, sind im Mittel mit niedrigeren CD4-Zellen bei Diagnosestellung assoziiert, als Teste im ambulanten Bereich (340 vs. 456/ $\mu$; $p=0,003$ ).

Schlussfolgerung: Um HIV-Infizierte auch in den ostdeutschen Bundesländern frühzeitig (d.h. mit $\mathrm{CD}_{4}>500 / \mu \mathrm{l}$ ) durch einen Test zu erreichen, sollte das Testangebot im ambulanten Bereich ausgebaut werden. Dazu gehört neben einem strukturierten Testangebot in der niedergelassenen Praxis - z.B. im Rahmen des Gesundheitschecks-35 - auch ein erweitertes Testangebot durch die AIDS-Hilfe, da hier im Rahmen des Studienprojektes mit durchschnittlich 585 CD4-Zellen/ $\mu \mathrm{l}$ die frühzeitigsten Diagnosen mit der besten Langzeitprognose beobachtet wurden. Die AIDS-Hilfe hat jedoch in unserem Studienkollektiv nur 3\% der diagnostisch relevanten HIV-Teste veranlasst. Zukünftige prospektive Studien sollten untersuchen, inwieweit niederschwellige Testangebote ohne obligate ärztliche Aufklärung eine Verbesserung der Testbereitschaft in Hochrisikogruppen erreichen können.

PS166

X-Tab: Nicht-interventionelle Langzeitstudie mit Omalizumab bei schwerem Asthma in Deutschland

Jens Schreiber'; Claudia Mailänder²

'Otto-von-Guericke-Universität mit Universitätsklinikum Magdeburg, Magdeburg; ${ }^{2}$ Novartis Pharma GmbH, Nürnberg

Einleitung: Ziel der nicht-interventionellen Studie X-Tab war die erstmalige Erhebung von deutschlandweiten prospektiven Daten aus der Praxisroutine zur Asthmakontrolle, der asthmabezogenen Lebensqualität und der Sicherheit während einer Langzeittherapie mit Omalizumab (OMA) bei schwerem allergischen Asthma (SAA).

Methodik: Einschlusskriterien waren eine SAA-Diagnose unter Ausschluss der Komorbidität COPD und mindestens eine OMA Verordnung bei der Eingangsvisite. Über einen Zeitraum von 3 Jahren wurde der Asthma-Kontrollstatus per Asthma Control Questionnaire (ACQ6) sowie die asthmabezogene Lebensqualität mittels Mini-Asthma Quality of Life Questionnaire (Mini-AQLQ) dokumentiert und die Exazerbationen erfasst.

Ergebnisse: 153 Erwachsene nahmen an der Studie teil ( $\varnothing$ 49.0 Jahre, $60.5 \%$ Frauen, $\varnothing$ Krankheitsdauer 15,5 Jahre). Die durchschnittliche Beobachtungsdauer betrug 901,6 Tage, 90 Patienten schlossen die Studie planmäßig nach 3 Jahren ab. 91,7\% der Patienten erhielten bereits vor Einschluss eine OMA Therapie ( $\varnothing$ Therapiedauer (SD) 3,1 $(2,6)$ Jahre vor Beobachtungsbeginn). Nach Beobachtungsbeginn wurden nur geringe Veränderungen in der Asthmakontrolle und der Lebensqualität verzeichnet - die Mittelwerte von ACQ6 lagen im gesamten Zeitraum zwischen 2,0 und 1,7. Der mittlere Mini-AQLQ lag zwischen 4,5 und 5,0; darüber hinaus war die annualisierte Exazerbationsrate konstant $<0,6 /$ Jahr, über die gesamte Studiendauer blieben mehr als zwei Drittel der Studienteilnehmer ohne schwere Exazerbation. Diese Ergebnisse sind vergleichbar mit den Daten einer retrospektiven Studie von Tzortzaki et al. (1), dort betrug die jährliche Exazerbationsrate 0,66 nach 4 Jahren anti-lgE Behandlung. Ebenso wurde bereits in $M$. Schatz et al. (2) gezeigt, dass ein Mini-AQLQ $>4,7$ mit einer geringen Exazerbationsrate assoziiert ist.

Schlussfolgerung: Diese Daten zeigen, dass Omalizumab auch unter Alltagsbedingungen über einen längeren Zeitraum eine stabile Asthma-Kontrolle sowie eine gleichbleibende asthmabedingte Lebensqualität bei Patienten mit schwerem allergischem Asthma gewährleisten kann.

(1) PPT 2012; 25: 77-82

(2) Am J Manag Care 2008;14(4):206-211 
PS167

Produktprobleme bei Insulinpumpen und deren Zubehör Analyse der 2005-2017 vom BfArM veröffentlichten Kundeninformationen

\section{Rüdiger Siekmeier}

Pharmazeutisches Institut der Universität Bonn, Bonn

Einleitung: Vermarktung und Marktüberwachung von Medizinprodukten werden in Europa durch Direktiven (z. B. The European Directive 93/42/EEC) geregelt. Bei Vorkommnissen und korrektiven Maßnahmen (Field Safety Corrective Action, FSCA) müssen die Hersteller diese den zuständigen nationalen Behörden (Competent Authority (CA); Deutschland: Bundesinstitut für Arzneimittel und Medizinprodukte (BfArM)) melden und die Kunden über Kundeninformationen (Field safety notice, FSN) informieren, die auch den Behörden zur Verfügung gestellt werden. Ziel der Studie war die Untersuchung von auf der Homepage des BfArM veröffentlichten FSN bei FSCA zu Insulinpumpen und deren Zubehör (Reservoire/Cartridges, Infusionssets) in Hinblick auf Produktprobleme, damit einhergehende Risiken sowie Art der FSCA.

Material und Methoden: Analysiert wurden die von Anfang 2005 bis November 2017 auf der Homepage (http://www.bfarm.de/DE/Medizinprodukte/riskinfo/kundeninfo/functions/kundeninfo-node.html) veröffentlichten FSCA und FSN.

Ergebnisse: Unter 10300 seit 2005 publizierten FSCA fanden sich 53 FSCA (36 Pumpen, 17 Zubehör). Typische Fehlerbilder bei Pumpen waren Komponentenfehler, Softwarefehler und Fehler der Gebrauchsanweisung, die zu Pumpenausfall, Unter- oder Überdosierung von Insulin, Alarmfehlern und Bedienfehlern führten, während sich bei Zubehör meist Leckagen/Ablösungen und Kanülenfehler mit Insulinunterdosierung oder Verletzung fanden. Die Fehlerbilder waren hinreichend beschrieben, jedoch war die zugrundeliegende Fehlerursache (z. B. Produktionsfehler, Komponentenfehler, Verschleiß, Softwarefehler) häufig nur aus dem Kontext identifizierbar. Nur in wenigen Fällen fanden sich Angaben zu aufgetretenen Patientenschädigungen. Häufigste korrektive Maßnahmen waren bei Pumpen Kundeninformationen mit teils umfangreichen Handlungsanweisungen (36) und Rückruf (19, einschl. Teile; Kundeninformation obligat), bei Zubehör Kundeninformationen (17) und Rückruf (10; Kundeninformation obligat).

Schlußfolgerungen: FSCA zu Insulinpumpen und deren Zubehör stellen aufgrund ihres Gefährdungspotentials bei Produktmängeln eine wichtige Produktgruppe dar. FSN zu Pumpen waren, bedingt durch die höhere Komplexität der Fehlerbilder und Anwendermaßnahmen, ausführlicher als solche zu Zubehör. Insulinpumpen und Zubehör unterscheiden sich deutlich in Hinblick auf Fehlerbilder (z. B. zu Insulinunterdosierung/Insulinüberdosierung führende Batterie- und Batteriefachfehler, Motorfehler, Softwarefehler und Gebrauchsanweisungsfehler bei Pumpen vs. Leckagen/Ablösungen und Kanülenfehler mit Insulinunterdosierung oder Verletzung bei Zubehör) sowie zu treffende Maßnahmen (z. B. bei Pumpen Kundeninformationen mit teils umfangreichen Handlungsanweisungen und Rückruf vs. Kundeninformationen und Rückruf/Vernichtung bei Zubehör). Aufgrund der Bedeutung der FSN zur Risikoverminderung bei FSCA sollten Form und Inhalt der FSN weiter verbessert werden.
PS168

Medical Apps - welche Rolle spielen Produktfehler? Analyse der 2005-2017 vom BfArM veröffentlichten Kundeninformationen

Rüdiger Siekmeier'; Jürgen Hannig'; Tanja Grammer²; Winfried März ${ }^{3}$

${ }^{1}$ Pharmazeutisches Institut der Universität Bonn, Bonn; ${ }^{2}$ Medizinische Fakultät Mannheim der Universität Heidelberg, Mannheim; ${ }^{3}$ Synlab Holding Deutschland GmbH, Mannheim, Mannheim

Einleitung: Medical Applications (Apps) bzw. Gesundheits-Apps finden zunehmend Verbreitung. Mehr als 150000 Healthcare-bezogene Apps sind bekannt, die den verschiedensten Zwecken dienen, z. B. Kommunikation mit Health Care Providern, Datenanzeige und Aufzeichnung, Alarmabgabe und Medikamenteneinnahme, aber auch Prävention, Therapie und Behandlung. Handelt es sich gemäß ihrer Zweckbestimmung um Medizinprodukte, sind Vermarktung und Marktüberwachung geregelt und Vorkommnisse und korrektive Maßnahmen (Field Safety Corrective Action, FSCA) müssen den zuständigen nationalen Behörden (Deutschland: Bundesinstitut für Arzneimittel und Medizinprodukte, BfArM) gemeldet werden. Diesem obliegt die Erfassung und wissenschaftliche Bewertung der Vorkommnisse und korrektiven Maßnahmen. Deren Durchführung wird durch die Länderbehörden überwacht. Im Falle von FSCA erfolgt die Veröffentlichung mit der zugehörigen Kundeninformation (Field Safety Note, FSN) auf der BfArM-Homepage (http://www.bfarm.de/DE/ Medizinprodukte/riskinfo/kundeninfo/functions/kunden info-node. html). Ziel dieser Studie war die Analyse der vom BfArM publizierten FSN zu Medical Apps in Hinblick auf Fehlerbilder und korrektive Maßnahmen.

Material und Methoden: Analysiert wurden alle 2005 bis Ende November 2017 vom BfArM publizierten FSCA und FSN zu Medizinprodukten und In-vitro Diagnostika.

Ergebnisse: Unter 10300 seit 2005 publizierten FSCA fanden sich 8 zu Medical Apps, die erste im Jahr 2011 zur Berechnung eines Rheumatologiescores durch Ärzte und 7 weitere seit 2014 zum Gebrauch durch diabetische Patienten (Laienanwender). Typische Fehlerbilder waren fehlerhafte Berechnung der Scores zur Berechnung der Rheumaaktivität (1) bei professionellen Anwendern sowie fehlerhafte Bolusvorschläge bzw. Bolusberechnungen (5), falsche Insulindosierungen $(n=2)$ und Angabe fehlerhafter Werte des aktiven Insulins $(n=2)$ bei Patienten, die zu fehlerhafter Einschätzung der Rheumaaktivität (1) und schweren gesundheitlichen Beeinträchtigungen bzw. Hypoglykaemie (4) führen können. Es erfolgten explizite Maßnahmen (Mehrfachnennung; z. B. Deaktivierung der Cloud (1), Deaktivierung der App (1), Software-Upgrades (7, z. T. automatisch)) und Anweisungen (z. B. Löschen der App, Kontrolle der Boluseinträge, Einstellung der Nutzung der Bolusvorschlagfunktion, Einstellung der Nutzung der Kohlenhydrat-/Insulinwerte) zur Risikominimierung.

Schlußfolgerungen: Obwohl bislang kaum FSCA zu Medical Apps vorliegen sind diese aufgrund ihrer medizinischen Relevanz (z. B. Hypoglykaemie) vor allem für Laienanwender von Bedeutung. Zunehmende Verbreitung und Bekanntheit ihrer Eigenschaft als Medizinprodukte, z. B. durch Aufklärung durch Wissenschaft, BfArM und andere, kompetente Behörden (z. B. U. S. Food and Drug Administration, FDA, Medicines and Healthcare Products Regulatory Agency, MHRA) sollten mit einer höheren Anzahl von Meldungen und einer weiteren Qualitätsverbesserung der Medical Apps einhergehen. 


\section{PS169}

\section{WannaCry und Co.: Wie steht es um die Cybersicherheit von Medizinprodukten? Analyse der vom BfArM 2017 publizierten Kundeninformationen}

\author{
Rüdiger Siekmeier'; Jürgen Hannig'; Tanja Grammer²; Winfried März ${ }^{3}$ \\ ${ }^{1}$ Pharmazeutisches Institut der Universität Bonn, Bonn; ${ }^{2}$ Medizinische \\ Fakultät Mannheim, Universität Heidelberg, Mannheim; ${ }^{3}$ Synlab Holding \\ Deutschland GmbH, Mannheim, Mannheim
}

Einleitung: Die Europäische Direktive 93/42/EEC reguliert Vermarktung und Marktüberwachung von Medizinprodukten in der Europäischen Union. Bei Vorkommnissen und korrektiven Maßnahmen im Feld (Field safety corrective action, FSCA) müssen die Hersteller die zuständige Behörde (Competent Authority, CA; D: BfArM) und die Öffentlichkeit mittels Kundeninformation (Field safety note, FSN) informieren. Angaben zu FSCA sowie die zugehörige FSN werden auf der BfArM Homepage veröffentlicht. 2017 traten durch Schadsoftware, insbesondere "WannaCry", erhebliche wirtschaftliche Schäden auf. Ziel dieser Studie war die Untersuchung von auf der Homepage des BfArM veröffentlichten FSCA auf das Vorliegen von Meldungen in Verbindung zur Cybersicherheit von Medizinprodukten.

Methoden: Sämtliche vom BfArM im Jahr 2017 (bis Ende November) veröffentlichten FSN (http://www.bfarm.de/DE/Medizinprodukte/ riskinfo/kundeninfo/functions/kundeninfo-node.html) wurden auf Inhalte bezüglich Cybersicherheit untersucht.

Ergebnisse: Bis Ende November 2017 wurden 832 FSCA/FSN publiziert von denen 11 die Cybersicherheit von Medizinprodukten betrafen. Bei den Produkten handelte es sich um Geräte aus den Bereichen Radiologie/MRT/Bestrahlungsmedizin (8), Kardiologie/Telemetrie (2) und Reinigung/Desinfektion (1), die von insgesamt 3 Herstellern produziert wurden. In 10 Fällen wurden von den Herstellern potentielle Probleme durch Schadsoftware (mehrfach "WannaCry") angegeben (nur in 1 Fall wurde der Befall eines Gerätes berichtet); in 1 weiteren Fall beschrieb der Hersteller ein Problem durch Programme zur Bekämpfung von Schadsoftware, die nicht infizierte Gerätedateien blockierten. Mögliche Fehlerbilder waren eine Beeinträchtigung der Gerätefunktion bis hin zum Ausfall. Das Auftreten von Patientenschäden wurde von den Herstellern meist ausgeschlossen bzw. es erfolgten Angaben, daß solche bislang nicht vorlagen. Zur Problembeseitigung erfolgten in allen Fällen Software-Upgrades (diese wurden von den Herstellern z. T. automatisch durchgeführt) sowie als Interimsmaßnahme die dringende Empfehlung der Errichtung einer Firewall bzw. einer Trennung der Geräte vom Internetzugang.

Diskussion: Die zunehmende Verbreitung komplexer softwaregestützter Medizinprodukte geht mit dem erhöhten Risiko eines Befalls durch Schadsoftware einher. Die Häufung von die Cybersicherheit betreffenden FSCA bei einzelnen Herstellern ist am ehesten Folge der Verwendung gleichartiger Softwarekonfigurationen bei verschiedenen Geräten des gleichen Herstellers. Zwar ist die 2017 beobachtete Häufung derartiger Meldungen gegenüber den Vorjahren Folge des Auftretens von "WannaCry", doch sollte diesem Problem auch nach Beseitigung der zugrundeliegenden Softwareschwachstellen weiter Aufmerksamkeit gewidmet werden. Verbreitung und Publikation der FSN bei FSCA zu Medizinprodukten tragen wesentlich zur Minimierung des durch Schadsoftware hervorgerufenen Risikos bei.
PS170

Patients' views on the impact of multi-drug-resistant bacterial microorganisms (MDRO) in end-of-life care - A qualitative study using principles of Grounded Theory.

Alexander Sturm ${ }^{1}$; Christoph Ostgathe ${ }^{2}$; Stephanie Stiel ${ }^{3}$; Maria Heckel ${ }^{2}$; Franziska A. Herbst ${ }^{3}$; Thomas Adelhardt ${ }^{4}$; Oliver Schöffski ${ }^{4}$; Christian Bogdan $^{5}$; Johanna Tiedtke ${ }^{6}$; Frieder R. Lang ${ }^{6}$; Cornel C. Sieber ${ }^{1}$

${ }^{1}$ Friedrich-Alexander-University (FAU) Erlangen-Nürnberg, Institute for Biomedicine of Aging \& Hospital of the Order of St. John of God Regensburg, Department of General Internal and Geriatric Medicine, Nürnberg, Regensburg; ${ }^{2}$ Friedrich-Alexander-University (FAU) Erlangen-Nürnberg, University Hospital Erlangen, Division of Palliative Medicine, Erlangen; ${ }^{3}$ Hannover Medical School, Institute for General Practice, Hannover; ${ }^{4}$ Friedrich-Alexander-University (FAU) Erlangen-Nürnberg, Division of Health Management, School of Business and Economics, Institute of Management, Nürnberg; ${ }^{5}$ Friedrich-Alexander-University (FAU) Erlangen-Nürnberg, University Hospital Erlangen, Institute of Microbiology - Clinical Microbiology, Immunology and Hygiene, Erlangen; ${ }^{6}$ Friedrich-Alexander-University (FAU)

Erlangen-Nürnberg, Institute of Psychogerontology, Nürnberg;

Objectives: The impact of infection and colonization with multi-drug-resistant bacterial microorganisms (MDRO) on patients in need of end-of-life care is mostly unknown and cannot be inferred from existing research data from a general population. Therefore, an interdisciplinary team of nursing scientists, psychologists, health economists and physicians realized "M-EndoL - MRSA in End-of-Life Care". M-EndoL aims at developing a patient-, family- and team-centered approach to handle MDRO in end-of-life care taking into account the needs of hospitalized patients, family caregivers, staff members and the needs of healthcare institutions as well. The present study is a subproject that focuses on patients' reports of their experienced impact of MDRO and aims to build a theoretical framework on the impact of MDRO in end-of-life care from the patients' point of view.

Methods: The study was performed in two study centers (01/2014 06/2016) with two periods of data assessment and analysis: interviews and focus group discussion. Using a semi-structured questionnaire, 43 patients were interviewed in order to explore the consequences of MDRO regarding information, communication, ailments, therapy and contact precautions. Interview findings were used to guide the focus group discussion conducted with five patients and three (professional) representatives in order to deepen the insights into the physical, emotional and social consequences as well as the patients' dealing with stigmatization. Verbatim transcripts of interviews and focus group discussion were inductively analyzed using principles of Grounded Theory.

Results: The impact of MDRO on patients in end-of-life care varies widely. It ranges from no impact to individually experienced consequences relating to the patient's physical constitution, social-life and emotional state. The impact often depends on the patient's health status and his MDRO-case-history, the main diagnosis and the family situation. As they experience the impact, patients cope with this situation as individuals.

Conclusion: The results suggest individual approaches when dealing with geriatric and palliative care patients with MDRO. Team members and institutional stakeholders should be aware that patients might be upset and even burdened by positive MDRO findings and the required contact precautions. Patients' suggestions imply that they would benefit from comprehensible information, empathic communication and individual support. Therefore, recommendations in German language, based on the results of all four subprojects of $\mathrm{M}$-EndoL were developed and published to realize a more individualized end-of-life 
care: "Zum Umgang mit multiresistenten Erregern (MRE) in der Versorgung am Lebensende im Krankenhaus."

[Download: https://www.dgpalliativmedizin.de/images/RZ_170213_ MRE_online.pdf]

Acknowledgement: The project is funded by the Federal Ministery of Education and Research (BMBF 01GY1314). There are no conflicts of interest to be reported.

\section{PS172}

Niedrige Exazerbationsrate nach Umstellung von COPD-Patienten auf eine LABA/LAMA Fixdosiskombination: Eine Analyse der DACCORD-Studie

Heinrich Worth ${ }^{1}$; Carl-Peter Criée ${ }^{2}$; Peter Kardos ${ }^{3}$; Nadine Lossi ${ }^{4}$; Claus Franz Vogelmeier ${ }^{5}$; Roland Buhl ${ }^{6}$

${ }^{1}$ Facharztforum Fürth, Fürth; ${ }^{2}$ Evangelisches Krankenhaus Göttingen Weende, Bovenden; ${ }^{3}$ Zentrum für Pneumologie, Allergie, Somnologie an der Klinik Maingau, Frankfurt; ${ }^{4}$ Novartis Pharma GmbH, Nürnberg; ${ }^{5}$ Universitätsklinikum Marburg, Marburg; ${ }^{6}$ Universitätsmedizin Mainz, Mainz

Einleitung: Die nicht-interventionelle DACCORD Studie dokumentiert Daten von COPD Patienten, welche in Allgemein- und Facharztpraxen deutschlandweit rekrutiert wurden. Hier berichten wir Ergebnisse aus dem ersten Beobachtungsjahr von 3084 Patienten, welche eine LABA/LAMA-Fixdosiskombination (-FDC) als Bestandteil ihrer COPD-Dauertherapie erhielten (LABA/LABA-FDC) sowie von $980 \mathrm{~Pa}-$ tienten, die keine LABA/LAMA-FDC im Rahmen ihrer Behandlung erhielten (Vergleichsarm).

Methoden: Patienten, bei denen der Beginn oder die Änderung der bestehenden COPD-Dauertherapie erfolgte, wurden in die Studie aufgenommen. Daten zu Exazerbationen und COPD-Medikation wurden bei Einschluss und in Folge alle drei Monate erhoben.

Ergebnisse: In der LABA/LAMA-FDC Gruppe erhielten 86,3\% der Patienten ausschließlich eine LABA/LAMA-FDC; 9.5\% zusätzlich ein ICS. Patienten im Vergleichsarm wurden mit LABA oder LAMA $(43,6 \%)$, einer Dreifachtherapie aus LABA+LAMA+ICS $(24,9 \%)$, der Kombination aus LABA oder LAMA plus ICS $(14,2 \%)$ oder LABA+LAMA $(11,3 \%)$ therapiert. Viele Patienten berichteten keine Exazerbationen in den 6 Monaten vor Einschluss ( $75,7 \%$ vs. $74,8 \%$ LABA/LAMA-FDC vs. Vergleichsarm). Im ersten Beobachtungsjahr blieben $74,7 \%$ vs. $72,1 \%$ der Patienten exazerbationsfrei (Exazerbationsraten 0,284 [95\% Cl 0,258; 0,312] und 0,343 [0,296; 0,398]). Der Großteil der Patienten ohne Exazerbationshistorie blieb auch im Folgejahr frei von Exazerbationen ( $78,6 \%$ vs. $78,3 \%)$. Patienten mit $\geq 1$ Exazerbation in den 6 Monaten vor Einschluss waren in der LABA/LAMA-FDC Gruppe häufiger frei von Exazerbationen als Patienten im Vergleichsarm (62,8\% vs. $53,1 \%, p=$ 0,029 ).

Schlussfolgerung: In dieser Population war die Exazerbationsrate der LABA/LAMA-FDC Gruppe (9,5\% der Patienten mit additivem ICS) somit ähnlich niedrig wie im Vergleichsarm ( $>35 \%$ der Patienten mit additivem ICS). Diese Daten bestätigen die Ergebnisse randomisierter klinischer Studien zur Wirksamkeit von LABA/LAMA Fixdosiskombinationen bei Patienten mit COPD.
PS173

Sprechen wir darüber - Kommunikation im deutschen Rettungsdienst

Matthias Zimmer'; Daria Czarniecki ${ }^{2}$; Stephan Sahm

${ }^{1}$ Ketteler Krankenhaus Offenbach, Offenbach; ${ }^{2}$ Goethe-Universität, Frankfurt;

Fragestellung: Kommunikation ist der Klebstoff der Teamarbeit. Unter dem Druck der Notfallmedizin kann sie der entscheidende Faktor im Überleben des Patienten sein. Doch wie ist es um die Selbstwahrnehmung im Bereich der Kommunikation im deutschen Rettungsdienst bestellt?

Methodik: Mitarbeiter im Rettungsdienst aus ganz Deutschland nahmen an der anonymen und freiwilligen Onlinebefragung teil.

Ergebnis: Die Teilnehmer (TN, w=17,7\%, $\mathrm{m}=82,3 \%$ ) waren Notärzte $\mathrm{n}=200$, Rettungsassistenten $\mathrm{n}=380$ und Notfallsanitäter $\mathrm{n}=131$, im Mittel 35,9 $\pm 10,5$ Jahre alt und hatten 12,5 $\pm 9,4$ Jahre Berufserfahrung. $53,2 \%$ (KI 49,5-56,9) hatten großes Interesse am Thema Kommunikation. Bei Übergaben vergessen $81,3 \%(\mathrm{KI} 78,3-84,1)$ selten mitgeteilte Informationen und $78,9 \%(\mathrm{KI} 75,8-81,9)$ selten Informationen zu übergeben. Bei übernommenen Aufgaben wiederholen 57\% die Aufgabenstellung selten / nie laut. Bei erledigten Aufgaben sagen 26,6\% dies selten / nie laut an. Stress sorgt bei den TN dafür, dass 5,4\% (KI 3,8-7,3) oft etwas verwechseln, 8,9\% (KI 6,9-11,2) sich oft verhören, sich $18,5 \%$ (KI 15,7-21,6) oft unpräzise ausdrücke, $28,0 \%$ (KI 24,8-31,5) oft weniger kommunizieren und sich 8,5\% (KI 6,5-10,8) im Ton vergreifen. Die Auswirkungen der Kommunikationsfehler führen bei $85,1 \%$ (KI 82,2$87,7)$ zu schlechten Gefühlen, 37,6\% (KI 34,0-41,4) schämen sich, 31,3\% (KI 27,8-34,9) haben Angst vor Sanktionen und für $11,4 \%$ (KI 9,1-14,0) ist es nicht schlimm. Scham besteht eher bei Unerfahrene als bei Erfahrene ( 51 vs. 31\%). Sanktionen werden eher von Nicht-Ärzten als von Arzt befürchtet (35\% vs. $18 \%$ ). Die Ursachen für schlechte professionelle Kommunikation sehen $83,0 \%$ (KI 80,0-85,7) in den Charakterzügen der Kollegen, $66,9 \%$ (KI 63,2-70,3) im Führungsverhalten, 56,9\% (KI $53,2-60,6)$ in der Arbeitsorganisation, 56,1\% (KI 52,3-59,8) mangelnde Fortbildungsmöglichkeiten und $49,7 \%(\mathrm{KI} 46,0-53,5)$ in den Charakterzügen der eigenen Person. Tendenziell galt je älter der TN desto eher war Führungsverhalten ursächlich und eher Nicht-Ärzte sehen einen Mangel bei den Fortbildungsmöglichkeiten. Eine Kommunikation über vorgefallene Fehler findet bei 2,0\% (KI 1,1-3,3) nicht, bei $45,9 \%(\mathrm{KI}$ 42,1-49,6) mit Vorgesetzen, bei 90,2\% (KI 87,7-92,2) mit Kollegen und bei $83,1 \%$ (KI 80,2-85,8) mit Freunde statt. 43,7\% (KI 39,9-47,4) wünschen eine Supervision zur Kommunikation mit einem Mediator. 96,3\% (KI 94,6-97,6) wünschen sich eine gemeinsame Fortbildung von Ärzten und Nicht-Ärzten. Mit Standards möchten 43,0\% (KI 39,4-46,8) bei der allg. Arbeit und $45,4 \%(\mathrm{KI} 41,7-49,1)$ bei Übergaben arbeiten. $57,1 \%(\mathrm{KI}$ $53,3-60,8)$ wünschen ein regelmäßiges Kommunikationstraining und für $79,7 \%(\mathrm{KI} 76,6-82,7)$ sollte es Teil der Berufsausbildung sein.

Schlussfolgerung: In der Fort- und Ausbildung aller Personen im Rettungsdienst sollte die Kommunikation im Team und bei Übergaben einen größeren Raum einnehmen. Informationsübergaben sollten in einem geschützten Raum stattfinden. Der Umgang mit der Kommunikation sollte professionalisiert werden. 
PS174

Überraschendes aus der Versorgungsforschung: Kardiologische

Praxen ohne Angina pectoris, gastroenterologische ohne Kolonadenome und andere Kuriositäten

Waltraud Zopf; Simon Dally

AOK Baden-Württemberg, Stuttgart

Einleitung: Diagnosen aus stationärer und ambulanter Versorgung können zu Versorgungsforschungszwecken genutzt werden. Bei der Verwendung dieser Sekundärdaten und deren Interpretation gibt es einige Fehlerquellen zu berücksichtigen. Im Rahmen von Versorgungsanalysen sind Versorgungslücken aufgefallen, die sich medizinisch nicht erklären lassen. Als Ursachen hat sich eine bisher kaum bekannte Fehlerquelle herausgestellt: die Schlagwortsuche der Praxissoftware (PVS). In der ambulanten vertragsärztlichen Versorgung nutzen Ärzte bei der Dokumentation von Diagnosen häufig die in der Software hinterlegte Schlagwortsuche. Dieser, auf dem alphabetischen Verzeichnis des DIMDI basierenden Thesaurus koppelt die Vielzahl von medizinischen Fachbegriffen an die begrenzte Anzahl von ICD-10 Diagnosekodes. Dabei können inhaltlich sehr ähnliche Schlagwörter zu unterschiedlichen ICD-10 Codes führen, was schließlich Auswirkungen auf die Versorgungsforschung hat.

Methode: Im Rahmen von Analysen zur Versorgungsstruktur ausgewählter Patientengruppen, für die Entwicklung von Selektivverträgen gemäß §73c SGBV, zeigte sich eine auffällige praxisindividuelle Heterogenität in der Kodierung einiger Erkrankungen. Hier zeigen wir eine Auswahl an ureigenen fachspezifischen Diagnosen, die mit überraschender Häufigkeit nicht in entsprechenden internistischen Praxen dokumentiert sind.

Ergebnisse: Datenanalysen zeigen, dass 15\% der Gastroenterologen ausschließlich gutartige hyperplastische Polypen (K63.5) kodieren [und nie Adenome] und fast 20\% der kardiologischen Praxen keine Angina pectoris (I20.-). Anhand dieser und weiterer Beispiele können wir zeigen, dass eine wesentliche Ursache für das Problem in der Schlagwortsuche der PVS liegt. So führt allein die Verwendung der Begriffe "Kolonpolyp" oder "adenomatöser Kolonpolyp" zu unterschiedlichen ICD-10 Kodes. Dieses Problem tritt insbesondere bei Erkrankungen auf, die sich mit verschiedenen medizinischen Begriffen beschreiben lassen.

Diskussion: Im medizinischen Praxisalltag werden ähnliche Begriffe für die Beschreibung desselben Krankheitsbildes verwendet. Bei der alleinigen Verwendung der Schlagwortsuche der PVS kann es zu systematisch falscher Diagnosedokumentation kommen, weil die Software die Unterschiede der Kodes nicht transparent macht, und den Klartext eines ICD-10 Kodes nicht anzeigt. Die Häufung falscher Diagnosen kann dann Versorgungsanalysen, die auf Basis ambulanter Daten durchgeführt werden, erheblich beeinträchtigen. Ziel dieser Arbeit ist es Beispiele für diese Problematik und deren Relevanz aufzuzeigen. In der Literatur wird das Problem aktuell bisher kaum berücksichtigt. Die standardmäßige Anzeige des „Klartext“ eines ICD10 Kodes bei allen PVSen kann helfen, das Problem künftig zu reduzieren. Bei der Analyse von Sekundärdaten sollte diese Fehlerquelle berücksichtigt werden. 


\section{A}

Acciuffi, S. (Magdeburg), PS001

Adams, V. (Dresden), PS149

Adelhardt, T. (Nürnberg), PS170

Alban, F. (Heidelberg), PSO22

Albert, A. (Regensburg), PS082

Albert, C. (Potsdam), PS156

Albrecht, H. (Neumarkt), PS083

Altendorf-Hofmann, A. (Jena),

PS001

Ambrosch, A. (Regensburg), PS073

Anders, H. (München), YIA01

Anraths, J. (Düsseldorf), PS059

Apfelbacher, C. (Regensburg), PS153

Arens, C. (Magdeburg), PS109

Aschenbach, R. (Jena), PSO29

Aschenbrenner, E. (Regensburg),

PS032, PS090, PS099

Athan, E. (Geelong, VIC), PS143

Auerswald, T. (Chemnitz), YIA02

Auerswald, T. (Nürnberg), PS04

Avelino-Silva, T. (Sao Paulo), PS143

Babel, N. (Herne), PS024, PS100

Bachmann, O. (Hannover), PS064

Backs, J. (Heidelberg), PS022

Balkaner, F. (Oschersleben), PS060

Balzen, L. (Oberhausen), PS061

Barnacle, H. (Uxbridge), PS086,

PS098

Bauer, F. (Herne), PS024, PS100

Bauer, K. (Saarbrücken-Bischmis-

heim), PS100

Bauersachs, J. (Hannover), PS013,

PS110

Baumgart, P. (Münster), PS125

Bauschke, A. (Jena), PS001

Becker, I. (Köln), PS043, PS046

Beckmann, M. (Erlangen), PS148

Beer, M. (Ulm), PS003

Bengesser, S. (Graz), PS127

Bening, C. (Würzburg), $\mathrm{PS} 003$

Benzing, T. (Köln), PS043, PS046

Berglund, J. (Karlskrona), PS143

Bergmann, C. (Freiburg im

Breisgau), PS121

Bergmann, T. (Regensburg), PS088

Berliner, D. (Hannover), PS110

Berschneider, K. (Nürnberg), PS110

Bertsch, T. (Nürnberg), PS017

Bewarder, M. (Homburg), PS139

Bickel, C. (Mainz), YIA05

Bickel, C. (Koblenz), PS140

Biller, H. (Hannover), PS110

Birk, R. (Uxbridge), PS086, PS098

Blake, A., PS122

Blüher, M. (Leipzig), PS027

Boashie, U. (Dresden), PS165

Bode, C. (Freiburg), PS067, PS068

Bogdan, C. (Erlangen), PS170

Bogner, I. (Leipzig), PS048

Böhm, M. (Homburg), PS012, PS103

Bokemeyer, B. (Minden), PS155
Bollheimer, C. (Aachen), PS017

Bollig, C. (Freiburg), PS045

Bossaller, L. (Greifswald), PS080

Bowen, T. (Leeds), PS149

Bozec, A. (Erlangen), PS070

Braisch, U. (UIm), PS040

Brandes, A. (Berlin), PS155

Bräsen, J. (Hannover), PS116

Braun, A. (Erlangen), PS083

Brealey, N. (Uxbridge), PS086, PS098

Brefka, S. (Ulm), PS045

Brinkers, M. (Magdeburg), PS101

Brinkkötter, P. (Köln), PS043

Broscaru, L. (Oberhausen), PS061,

PS124

Brünger, M. (Berlin), PS141

Bruns, T. (Jena), PS029

Brünsing, J. (Aachen), PS074

Brzoska, M. (Fankfurt), PS063

Budd Haeberlein, S. (Cambridge,

MA), PS132

Buhl, R. (Mainz), PS091, PS172

Burkhardt, H. (Mannheim), PS036, PS037

Burmester, G. (Berlin), PS015

Butter, C. (Bernau), PS156

C

Campora, L. (Wavre), PS142, PS143 Canbay, A. (Magdeburg), PS081

Cariou, B. (Nantes), PS102

Cato, A. (Karlsruhe), YIA10

Chatzikyrkou, C. (Magdeburg),

PS120, PS121

Chatzitolios, A. (Thessaloniki),

PS084, PS085

Chen, T. (Cambridge, MA), PS132

Cheng, Q. (Berlin), YIA08, PS031

Chiao, P. (Cambridge, MA), PS132

Chira, R. (Cluj-Napoca), PS038

Chlibek, R. (Hradec Kralove,), PS143

Christa, M. (Würzburg), PS003

Churin, Y. (Gießen), PS078

Cornberg, M. (Hannover), PS014,

YIA07

Criée, C. (Bovenden), PS172

Croner, R. (Magdeburg), PS001

Cunningham, A. (Sydney, NSW), PS143

Curtis, R., PS122

Czarniecki, D. (Frankfurt), PS173

\section{D}

Dalkner, N. (Graz), PS127

Dallmeier, D. (Ulm), PS040, PS045

Dally, S. (Stuttgart), PS174

Danne, T. (Hannover), PS102

Dathe, H. (Göttingen), YIA04

De Looze, F. (Brisbane), PS142

de With, K. (Dresden), PS069

Dederer, J. (Homburg), PS103
Deeb, J. (Magdeburg), PS152

Deiters, B. (Düsseldorf), PS155

Delannois, F. (Wavre), PS142

Demirci, C. (Regensburg), PSO02

Demuth, D., PS122

Denkinger, M. (Ulm), PS040, PS045

Didaggelos, T. (Thessaloniki),

PS084, PS085

Diebold, S. (Aachen), PS074

Diepolder, I. (Aachen), PS074

Dieterich, W. (Erlangen), PS051,

PS056

Dietrich, C. (Stolberg). PS136

Diez-Domingo, J. (Valencia), PS142,

PS143

Dignass, A., PS122

Diller, M. (Bad Nauheim), YIA09

Dobre, C. (Oberhausen), PS124

Dombrowski, F. (Greifswald), PS080

Dorbath, C. (Frankfurt), PS063, PS137

Downey, J. (Jacksonville, FL), PS143

Dransfield, M. (Birmingham), PS086

Duca, I. (Cluj-Napoca), PSO38

Dumbrava, D. (Magdeburg), PS104

Dumitrascu, D. (Cluj-Napoca), PS038

E

Eberlein-Gonska, M. (Dresden)

PS153

Eggebrecht, L. (Mainz), YIA05, PS140

Ehmann, C. (Pforzheim), PS112

Ehrenschwender, M. (Regensburg) PS026

Eisfeldt, C. (Magdeburg), PSO20

El Idrissi, M. (Wavre), PS142

El-Armouche, A. (Dresden), PS013

Elbelt, U. (Berlin), PS129

Elies, A. (Münster), PS125

Emrich, I. (Homburg), PS012, PS018,

PS033, PS105, PS123

Ernst, M. (Magdeburg). PS156

Ertl, G. (Würzburg), PS003

Eskofier, B. (Erlangen), PS052

Espinola-Klein, C. (Mainz), YIA05,

PS140

Essmann, J. (Hannover), PS064

Exeriede, G. (Cuxhaven), PS158

Eyermann, R. (München), PS106,

PS107, PS108, PS126

\section{F}

Faber, L. (Bad Oeynhausen), PS097

Farr, M. (Bad Oeynhausen), PS097

Feldmann, A. (München), PS153

Felix, S. (Greifswald), PS080

Fellendorf, F. (Graz), PS053, PS127,

PS130

Fenske, W. (Leipzig), PS128

Fietkau, R. (Erlangen), PS148

Fili, M. (London), YIA04

Fischer, J. (Mannheim), PS147
Fleck, M. (Regensburg), YIA09 Fliser, D. (Homburg), PS012, PS018, PS033, PS105, PS123, PS139

Flothow, D. (Münster), PS134

Fodor, D. (Cluj-Napoca), PS038

Foldenauer, A. (Aachen), PS074

Forster, C. (Regensburg), PS082

Frank, M. (Marburg), PS094

Franke, A. (Nürnberg), YIA02, PS039,

PS041

Franke, T. (Göttingen), YIA04

Frantz, S. (Würzburg), PSO03

Freiberger, E. (Nürnberg), YIA02,

PS039, PS041, PS042, PS055, PS164

Frey, N. (Kiel), PS097

Friebe, B. (Magdeburg), PS114

Friedrich, T. (Berlin), PS131

Fries, B. (Karlsruhe), $\mathrm{PS} 003$

Fries, C. (Leipzig), PS128

Frille, A. (Leipzig), PS004

Fritzsche, C. (Rostock), PS165

Froese, N. (Hannover), PS013

Frommer, K. (Bad Nauheim), PS050, PS065

Fuchs, A. (Dresden), PS155

Füldner, F. (Gera), PS115

G

Gastinger, I. (Magdeburg), PS005, PS154, PS159, PS162

Gattenlöhner, S. (Gießen), PS003

Gebauer, B. (Magdeburg), PSO05, PS162

Gensler, D. (Würzburg), PS003

Georgescu, A. (Regensburg), PS002

Gerhard, T. (Mönchsdeggingen),

PS040

Ghesquiere, W. (Victoria, BC), PS143

Gheuens, S. (Cambridge, MA), PS132

Giannoulaki, P. (Thessaloniki)

PS084

Gnemmi, V. (München), YIA01

Göbel, S. (Mainz), YIA05, PS140

Godeaux, O. (King of Prussia, PA),

PS143

Goebel-Stengel, M. (Rottweil)

PS129, PS131

Golsong, V. (Oberhausen), PS061

Gorfinkel, I. (Toronto, ON), PS143

Gößmann, H. (Regensburg), PS119

Gottfried, R. (Bad Oeynhausen),

PS145

Gotthardt, S. (Nürnberg), YIA02,

PS039, PS041

Gräb, J. (Köln), YIA10

Grammer, T. (Mannheim), PS168,

PS169

Granowski, D. (Magdeburg), PS109

Grenkowitz, T. (Berlin), PS010

Gröne, H. (Heidelberg), PS120, PS121

Gross, M. (München), PS161

Groth, A. (Wismar), PS155

Gröticke, J. (Bremen), PS158

Grotius, K., PS067, PS068 
Grund, A. (Hannover), PS013 Grützmacher, P. (Frankfurt), PS063, PS137

Gulba, D. (Oberhausen), PS061, PS124 Gutensohn, K. (Hamburg), PS047

\section{$H$}

Haag, F. (Hamburg), PS079 Haake, H. (Mönchengladbach), PS059

Haase, M. (Potsdam), PS156 Haase-Fielitz, A. (Bernau), PS156 Hack, C. (Erlangen), PS148 Haderer, M. (Regensburg), PS099 Hahne, S. (Berlin), PS015 Hain, J. (München), PS142, PS143 Haj-Yehia, E. (Aachen), PS074 Halloul, Z. (Magdeburg), PS023, PS109

Halpin, D. (Exeter), PS086

Hamm, C. (Graz), PS127, PS130

Hannig, J. (Bonn), PS168, PS169

Hardt, R. (Mainz), YIA05, PS140

Härdtner, C. (Freiburg), PS067, PS068

Harth, A. (Köln), PS072

Hartleb, R. (Graz), PS130

Hasenfuß, G. (Göttingen), YIA04

Haslinger, C. (Heidelberg), PSO22

Hather, G., PS122

Hauser, A. (Berlin), YIA08

Heckel, M. (Erlangen), PS170

Heine, G. (Homburg), PS012, PS018, PS033, PS0105, PS123, PS139

Heineke, J. (Mannheim), PS013 Heinemann, T. (King of Prussia, PA), PS143

Heinken, A. (München), YIA03

Helbig, S. (Dresden), PSO69

Herbst, F. (Hannover), PS170 Hergenröther, A. (Wiesbaden), PS160

Hermes, K. (Bremen), PS158 Hernandez Petzsche, M. (München), YIA01

Herrmann, H. (Erlangen), PS052, PS054, PS058, PS146, PS148

Herrmann, S. (Würzburg), PS003 Hertenstein, B. (Bremen), PS158 Hertling, S. (Markranstädt), PS006, PS007, PS008, PS009

Hess, T. (Saalfeld), PS05

Hesse, S. (Leipzig), PSO04

Heuchel, T. (Chemnitz), PS165

Hiepe, F. (Berlin), YIA08, PS015, PS031

Hilgendorf, I. (Freiburg im

Breisgau), PS067, PS068

Hiltl, S. (Nürnberg), PS110

Hippe, H. (Kiel), PS087

Hock, C. (Zürich), PS132

Hoffmann, A. (Magdeburg), PS08

Hofmann, A. (Nürnberg), YIA02,

PS041

Hofmann, T. (Berlin), PS129

Hohl, M. (Homburg), PS103
Höhler, T. (Recklinghausen), PS093 Hohlfeld, J. (Hannover), PS110 Hollenz, M. (Rödental), PS161 Hollstein, T. (Berlin), PS010 Hölscher, C. (Borstel), YIA10 Horstkotte, D. (Bad Oeynhausen) PS097, PS145

Hoyer, B. (Kiel), YIA08, PS015, PS031 Hoyer, J. (Marburg an der Lahn), PS077

Hu, K. (Würzburg), PS003

Huber, Y. (Mainz), PS151

Hübner, L. (Leipzig), PS128

Hülser, M. (Gießen), PS070, YIA09

Hummel, A. (Greifswald), PS080

Hüttemann, P. (Berlin), PS011, PS02

Hwang, S. (Taipei), PS143

I

Ingenabel, F. (Oberhausen), PS061 PS124

srael, H. (Halle), PS111

Ivan, M. (Pforzheim), PS112

J

Jacob, D. (Magdeburg), YIA06 Jacob, S. (Villingen-Schwenningen), PS157

Jäger, H. (Munich), PS165

Jander, A., PS067

Jechorek, D. (Magdeburg), PS109

Jenke, A. (Dresden), PS165 Jerrentrup, A. (Marburg), PS019

Jörres, A. (Köln), PS072

\section{K}

Kaczmarek, P. (Greifswald), PS080 Kahles, F. (Aachen), PS074 Kahlmeyer, A. (Erlangen), PS148 Kaiser, H. (Homburg), PS012 Kamm, M. (Köln), PS072 Kandulski, M. (Regensburg), PS088 Kanthak, H. (Esslingen), PS089 Kanzler, S. (Schweinfurt), PS093 Kapfhammer, H. (Graz), PS053 Kardos, P. (Frankfurt), PS172 Karlafti, E. (Thessaloniki), PS084 Karpouza, A. (Cuxhaven), PS158 Kaser, L. (Sulzbach-Rosenberg), PS090

Kassner, U. (Berlin), PS010

Kattih, B. (Mannheim), PS013 Katus, H. (Heidelberg), PS013, PS022 Katzenstein, J. (Magdeburg), PS159 Kazakov, A. (Homburg), PS103 Kedor, C. (Berlin), PS031 Keil, C. (Hannover), PS064 Keller, K. (Mainz), YIA05, PS140
Kemmler, W. (Erlangen), PS148 Kestler, A. (Regensburg), PS164 Khalid, M., PS122

Kiesswetter, E. (Nürnberg), PS049 PS055, PS057

Kimmann, M. (Hannover), PS014 Klaas, M. (Mainz, Germany), PS140 Klaus, J. (UIm), PS040

Kleinert, C. (Frankfurt), PS063 Klugmann, M. (Leipzig), PS048 Kob, R. (Nürnberg), PS017 Kobelt, P. (Berlin), PS131

Koch, A. (Aachen), PSO74

Kocheise, F. (Regensburg), PS002 Koljaja-Batzner, A. (Würzburg), PS113

Königsmann, J. (Berlin), PS011, PS021 Kontoninas, Z. (Veroia), PS084, PS085 Konturek, P. (Saalfeld), PS051 Korf-Klingebiel, M. (Hannover) PS013

Korhonen, T. (Tampere), PS143

Kornemann, J., PS068

Korsake, K. (Wernigerode), PS114

Kottmann, T. (Hamm), PS097, PS136

Kotzakioulafi, E. (Thessaloniki),

PS084

Kraft, M. (Berlin), YIA08

Krammer, M. (Pforzheim), PS112

Kraska, C. (Nürnberg), YIA02, PS041

Krause, K. (Germany), PS093

Krawczyk, M. (Homburg), PS103

Kretzschmar, A. (Leipzig), YIA03

Kropf, S. (Magdeburg), PS152

Krumpoch, S. (Nürnberg), PSO42

Kuhn, H. (Leipzig), PS004

Kümmel, A. (Mainz), PS091

Kumpert, C. (Berlin), PS015

Kunst, C. (Regensburg), PS016, PS032

PS090, PS099

Kunzmann, V. (Würzburg), PS093

Kurrle, A. (Wiesbaden), PS160

Kuss, B. (Bremen), PS158

L

Labenz, C. (Mainz), PS161

Labenz, J. (Siegen), PS161

Lahousen-Luxenberger, T. (Graz),

PS053

Lal, H. (King of Prussia, PA), PS143

Lammerding, D. (Berlin), YIA08

Lammert, F. (Homburg), PS103

Lamparter, H. (Mainz), YIA05, PS140

Lang, F. (Nürnberg), PS170

Langer, C. (Bremen), PS097

Langhof, H. (Berchtesgaden), PS108,

PS126

Laser, H. (Hannover), PS014

Laurentius, T. (Aachen), PS017

Lauterbach, M. (Trier), YIA05, PS140

Lebherz, C. (Aachen), PS074

Ledent, E. (Wavre), PS142

Leenen, E. (Köln), PS072

Lehle, K. (Regensburg), PS009
Lehmann, T. (Jena), PS029 Lehrke, M. (Aachen), PS074 Lennartz, C. (Homburg), PS018, PS139 Lennartz, S. (Homburg), PS105 Lepiorz, M. (Regensburg), PS073 Leschke, M. (Esslingen), PS089 Levin, M. (Aurora, CO), PS143 Leyh, R. (Würzburg), PSO03 Linke, A. (Dresden), PS149 Linz, D. (Homburg), PS103 Lippert, H. (Magdeburg), PS001, PS005, PS159, PS162

Lipson, D. (King of Prussia, Philadelphia), PS098

Lipson, D. (King of Prussia), PS086 Liu, D. (Würzburg), PSO03 Locantore, N. (King of Prussia), PS098

Lodes, U. (Magdeburg), YIA06 Loftus, E., PS122 Lomas, D. (London), PS098 Lopez-Fauqued, M. (Wavre), PS142 Lordick, F. (Leipzig), PS093 Lorenz, K. (Essen), PS003 Lossi, N. (Nürnberg), PS172 Lubgan, D. (Erlangen), PS148 Lüdde, M. (Kiel), PS087 Luo, Y. (Erlangen), PS070 Lutz, M. (Kiel), PS097

$M$

Ma, Q. (München), YIA01 Maasoumy, B. (Hannover), PS014, YIA07

Maderer, A. (Mainz), PS093

Madisch, A. (Hannover), PS092, PS161 Maget, A. (Graz), PS130

Mahfoud, F. (Homburg), PS103 Maier-Giebing, T. (Marburg an der Lahn), PS077

Maier-Stocker, C. (Regensburg), PSO02

Mailänder, C. (Nürnberg), PS166 Makowski, M. (Berlin), PS015 Malfertheiner, P. (Magdeburg), PS081

Mangge, H. (Graz), PS127, PS130

Manns, M. (Hannover), PS014, PS064, PS116, YIA07

Margaritidis, C. (Serres), PS085 Markus, L. (Greifswald), PS080 Martin, J. (Berlin), PS031 Martin, L. (Aachen), PS074 Marx, N. (Aachen), PS074 März, W. (Mannheim), PS010, PS168, PS169

Masaryk, V. (Gera), PS115 Mavromanoli, A. (Thessaloniki) PS084, PS085

May, M. (Düsseldorf), YIA03

Mayer, K. (UIm), PS040 Mayr, R. (Regensburg), PS075 McElhaney, J. (Sudbury, ON), PS142, PS143 
McNeil, S. (Halifax, NS), PS142, PS143 McSweeney, S. (London), PS044 Mederacke, I. (Hannover), PS116 Mederacke, Y. (Hannover), PS116 Mellou, M. (Nürnberg), YIA02, PS041 Mellwig, K. (Bad Oeynhausen), PS145

Melms, L. (Marburg), PS019 Mertens, P. (Magdeburg), PS120, PS121

Mertens, R. (Aachen), PS074 Mester, P. (Regensburg), PS117 Meyer, A. (Köln), PS043, PS046 Meyer, C. (Nürnberg), PS042 Meyer, F. (Magdeburg), PS001, PSO05, PS023, PS030, PS101, PS109, PS114, PS115, PS152, PS154, PS159, PS162, YIA06 Meyer, H. (Berlin), PS093 Meyer, J. (Erlangen), PS052, PS148 Michels, B. (Regensburg), PS075 Mickley, F. (Leipzig), PS048 Mihaljevic, A. (München), PS093 Mildner, T. (Wernigerode), PS114 Miron, I. (Cluj-Napoca), PS038 Mittag, J. (Magdeburg), PS104 Möhler, M. (Mainz), PS093 Mohrlang, C. (München), PS086, PS096

Mollenkopf, H. (Berlin), PS078 Möllmann, J. (Aachen), PS074 Molloy, W. (Cork, Co. Cork), PS044 Mönig, S. (Köln), PS093 Mosafer, A. (Weimar), PSO20 Mosli, M., PS122

Mross, T. (Cork, Co. Cork), PS044 Mühlbauer, V. (UIm), PS045 Müller, M. (Oberhausen), PS061, PS124 Müller, M. (Regensburg), PSO02, PS016, PS032, PS088, PS090, PS117, PS119, PS150

Müller, O. (Kiel), PS013

Müller, T. (Marburg), PS019, PS094 Müller-Ladner, U. (Gießen), PS050, PS065, PS070, YIA09

Müller-Schilling, M. (Regensburg), PS075, PS082, PS099

Münzel, T. (Mainz), YIA05, PS140

$\mathrm{N}$

Nagel, A. (Erlangen), PS083 Nagler, M. (Mainz), PS140, YIA05 Nair, R. (kochi), PS095 Nebe, T. (Mannheim), PS047 Neuling, F. (Magdeburg), YIA06 Neumann, E. (Gießen), PS065, PS070, YIA09

Neumann, E. (Bad Nauheim), PS050 Neurath, M. (Erlangen), PS051, PS052 PS054, PS056, PS058, PS, 146, PS148 Nickolaus, T. (Marburg), PS019, PS094 Nitsch, R. (Zürich), PS132 Nordbeck, P. (Würzburg), PS003 Nourbakhsh, M. (Aachen), PS017
Nowak, A. (Berlin), PS011, PS021 Nusser, C. (Regensburg), PS075

\section{0}

Oberländer, J. (Erlangen), PS052 O'Caoimh, R. (Galway), PS044 O'Gorman, J. (Cambridge, MA), PS132 Öhm, B. (Frankfurt), PS137 Okech, T. (Pforzheim), PS112 Oostvogels, L. (Wavre), PS143 Orlemann, T. (Erlangen), PS052, PS148

Ortmann, O. (Regensburg), PS088 Ostgathe, C. (Erlangen), PS170 Otto, R. (Magdeburg), PS005, PS162

\section{P}

Paar, W. (Berlin), PS144

Pagonas, N. (Herne), PS024

Parhofer, K. (München), PS144

Parigger, L. (Mannheim), PS036

Paschala, A. (Thessaloniki), PS084, PS085

Pascoe, S. (King of Prussia), PS098 Pauksens, K. (Uppsala), PS143 Paul, S. (The Woodlands), PS102 Pavel, V. (Regensburg), PS119 Pavenstädt, H. (Münster), PS134 Peschel, G. (Regensburg), PS082, PS117

Peters, J. (Magdeburg), PS121

Petersen, M. (Magdeburg), PS152

Peyrin-Biroulet, L., PS122

Pfau, G. (Magdeburg), PS101 Pfeiffer, B. (Schweinfurt), PS113

Pfirrmann, D. (Mainz), PS151

Pickering, J. (Christchurch), PS018

Pickert, L. (Köln), PS046

Pillai, M. (Kochi), PS028

Pilotto, A. (Genova), PS043

Pilz, R. (Graz), PS127, PS130

Pinzer, T. (Erlangen), PS056 Platzer, M. (Graz), PS053, PS, 127, PS130

Pletz, M. (Jena), PS165

Polidori, M. (Köln), PS043, PS046

Pollinger, K., PS032, PS090

Pollinger, K. (Regensburg), PS099

Pollock, K. (Berlin), YIA08

Postel, N. (Munich), PS165

Poudel, M. (Bad Oeynhausen), PS145

Prochaska, J. (Mainz), YIA05, PS140

Ptok, H. (Magdeburg), PS005, PS159, PS162

Puig-Barbera, J. (Valencia), PS143

Q

Queissner, R. (Graz), PS127, PS130
R

Radbruch, A. (Berlin), YIA08 Rafflenbeul, E. (Hamburg), PS135 Raithel, M. (Erlangen), PS083 Ratsch, B. (Berlin), PS155 Raulien, N. (Leipzig), PS027 Rayes, N. (Leipzig), PS128 Rehart, S. (Frankfurt), YIA09, PS050, PS065

Reichert, T. (Mainz), PS091 Reininghaus, E. (Graz), PS127, PS130 Reith, V. (Nürnberg), PS039, PS041, PS042

Reljic, D. (Erlangen), PS052, PS054, PS058, PS146, PS147

Rempe, H. (Nürnberg), PS039 Rempe, H. (Bamberg), PS055 Reuter, C. (Hannover), PS158 Reuter, S. (Münster), PS134 Ribback, S. (Greifswald), PS080 Rieger, A. (Graz), PS127 Riess, H. (Berlin), YIA03 Rimmele, M. (Nürnberg), PS164 Robra, B. (Magdeburg), PS156 Roderfeld, M. (Gießen), PS078 Roeb, E. (Gießen), PS078 Roehrig-Herzog, G. (Köln), PS047 Rohn, B. (Herne), PS024, PS100 Rolim, N. (Trondheim), PS149 Rolshoven, E. (Saarbrücken-Püttlingen), PS100

Rose, M. (Berlin), PS129, PS131 Rosenstengel, S. (Münster), PS125 Rößler, S. (Chemnitz), PS165 Rossol, M. (Leipzig), PS027 Rottmann, T. (Göttingen), YIA04 Ruck, I. (Leipzig), PS165 Rudolph, B. (Berlin), PS015 Ruppert, V. (Marburg), PS094 Rußwurm, M. (Marburg an der Lahn), PS077

Rybniker, J. (Köln), YIA10

\section{$S$}

Sabo, C. (Cluj-Napoca), PS038 Sabri, O. (Leipzig), PSO04 Sahm, S. (Offenbach), PS173 Salinger, T. (Würzburg), PS003 Sandermann, A. (Langenfeld), PS093

Sandoval, D. (Ann Arbor), PS074

Sandrock, A. (Cambridge, MA), PS132 Sauerbier, M. (Frankfurt), PS065 Sawhney, S. (The Woodlands), PS102 Schäfer, C. (Neumarkt), PS083 Schäfer, J. (Marburg), PS019, PS094 Scharnagl, H. (Berlin), PS010 Scharner, S. (Berliin), PS131 Schattenberg, J. (Mainz), PS151 Schell, R. (Heidelberg), PSO22 Schellong, S. (Dresden), YIA03 Scherr, F. (Mannheim), PS037 Schett, G. (Erlangen), PS070 Schiefer, J. (Magdeburg), PS120,
PS121

Schieffer, B. (Marburg), PS019 Schink, K. (Falkenstein), PS148 Schink, M. (Nürnberg), PS056 Schinköthe, C. (Leipzig), PS048 Schirmer, S. (Homburg), PS012 Schleenvoigt, B. (Jena), PS165 Schlosser, S. (Regensburg), PS099, PS150

Schmederer, Z. (Leipzig), PS149 Schmid, S. (Regensburg), PS002, PS075, PS119

Schmidt, H. (Magdeburg), PS104

Schmidt, L. (Münster), PS091 Schmidt, R. (Münster), PS134 Schmidt-Lucke, A. (Berlin), PS011, PS021

Schmidt-Lucke, C. (Berlin), PS011, PS021

Schmitt, R. (Hannover), PS116 Schmitt, V. (Mainz), PS140 Schmitz, J. (Hannover), PS116 Schneider, A. (Esslingen), PS089 Schneitler, S. (Solingen), PS165 Schoene, D. (Nürnberg), PS041 Schöffski, O. (Nürnberg), PS170 Scholtz, V. (Magdeburg), PS023 Schöne, D. (Nürnberg), YIA02, PS039 Schreiber, J. (Magdeburg), PS166

Schreiber, S. (Kiel), PS122 Schreiyäck, C. (Bad Nauheim), PS070 Schroll, S. (Braunschweig), PS093 Schroll, S. (Regensburg), PS073 Schuhmacher, C. (München), PS093 Schulten, R. (Witten), PS136 Schulz, A. (Berlin), PS031 Schulz, C. (Munich), PS165 Schulz, H. (Haldensleben), PS023 Schürholz, T. (Rostock), PS074 Schütte-Nütgen, K. (Münster), PS134 Schwappacher, R. (Erlangen), PS148 Schwarz, D. (Gießen), PS078 Seggewiss, H. (Schweinfurt), PS113 Seibert, F. (Herne), PS024, PS100 Seidler, T. (Göttingen), YIA04 Seiler-Mußler, S. (Saarlouis), PS018 Seiler-Mußler, S. (Homburg), PS033, PS105, PS123

Selejan, S. (Homburg), PS103 Selgrad, C. (Regensburg), PS088 Selgrad, M. (Regensburg), PSO02, PS075, PS088

Sellier, A. (Homburg), PS123 Sellmann, M. (Berlin), PS165 Sellmayr, M. (Neubiberg), YIA01 Settmacher, U. (Jena), PS001 Seyfarth, H. (Leipzig), PS004 Sieber, C. (Nürnberg), YIA02, PS017, PS039, PS041, PS042, PS049, PS055 PS057, PS164, PS170

Sieber, G. (Nürnberg), PS039 Siebler, J. (Erlangen), PS148 Siekmeier, R. (Bonn), PS167, PS168, PS169

Simon, P. (Mainz), PS151

Sirtl, S. (Regensburg), PS026 Sivanathan, V. (Mainz), PS093 
Skordos, L. (Cambridge, MA), PS132 Sommer, M. (Leipzig), PS027 Sossalla, S. (Regensburg), PS087 Soufi, M. (Marburg), PS094 Speer, T. (Homburg), PS103 Sproesser, G. (Konstanz), PS055 Spura, A. (Magdeburg), PS156 Spyra, K. (Berlin), PS141 Srikanth, V. (KOCHI), PS028, PS095 Stachon, P. (Freiburg im Breisgau), PS067, PS068

Stahl, M. (Essen), PS093

Stahr, T. (Jena), PS029

Stallmach, A. (Jena), PS029 Stegherr, A. (München), PS096, PS098 Stegmaier, A. (Schwabach), PS083 Steiger, S. (München), YIA01 Steinert, R. (Salzkotten), PS005, PS159, PS162

Steinhagen-Thiessen, E. (Berlin), PS010

Steinhoff, K. (Lepzig), PS004 Stengel, A. (Tübingen), PS129, PS131 Stergiou, I. (Thessaloniki), PS085 Stervbo-Kristensen, U. (Herne), PS024

Stiehler, M. (Dresden), PS069 Stiel, S. (Hannover), PS170 Störk, S. (Würzburg), PS003 Stoyanova, D. (Bad Oeynhausen), PS145

Stracke, B. (Karlsruhe), PS092 Strauß, T. (Nürnberg), PS049, PS057 Strotmann, J. (Kiel), PS003 Strungaru, E. (Magdeburg), PS030 Studiengruppe Primäres Magenkarzinom "“", .. (Magdeburg), PS154 Sturm, A. (Nürnberg, Regensburg), PS170

Sudau, M. (Magdeburg), PS104 Süßkind von Schwendi, M. (Regensburg), PS006, PS007, PS008, PS009 Suwelack, B. (Münster), PS134 Swierzewska, S. (Hamm), PS097 Szaroszyk, M. (Hannover), PS013 Szymczak, S. (Frankfurt), PS137

\section{T}

Tabberer, M. (Uxbridge), PS098 Tacke, F. (Aachen), PS074 Tamares da Silva, F. (Wavre), PS142 Tannapfel, A. (Bochum), PS093 Teege, S. (Hamburg), PS079 Tergast, T. (Hannover), PS014, YIA07 Tetzlaff, S. (Magdeburg), PS104 Theis, P. (Heidelberg), PS022 Thomas, A. (kochi), PS095 Thrum, S. (Leipzig), PS027 Thum, T. (Hannover), PS013 Thuss-Patience, P. (Berlin), PS093 Tiedtke, J. (Nürnberg), PS170 Tietz, E. (Erlangen), PS056 Tilgner, G. (Berlin), PS031 Tillmann, H. (Basel), PS110
Tönjes, A. (Leipzig), PS128 Torbahn, G. (Nürnberg), PS045, PS049, PS057

Toth, I. (Greifswald), PS080

Tröger, U. (Magdeburg), YIA06 Tsiklauri, L. (Bad Nauheim), PS050 Tsotoulidis, S. (Chalkidiki), PS085 Tziomalos, K. (Thessaloniki), PS085

U

Udelnow, A. (Magdeburg), PS109 Ungermann, E. (Regensburg), PS032 Untersteller, K. (Homburg), PS012, PS018, PS033, PS105, PS139 Utz, C. (Mainz), PS093

V

Van Buuren, F. (Olpe), PS145 van Gumpel, E. (Köln), YIA10 Vanden Abeele, C. (Wavre), PS143 Vesikari, T. (Tampere), PS143 Vogel-Claussen, J. (Hannover) PS110

Vogelmeier, C. (Marburg), PS172 Voigt-Radloff, S. (Freiburg), PS045 Völker, W. (Würzburg), PSO03 Volkert, D. (Nürnberg), PS049, PS055, PS057, PS164

Volpi, A. (Rome), PS143

von Arnim, U. (Magdeburg), PS081

von Hehn, C. (Cambridge, MA), PS132 von Rosenstiel, P. (Cambridge, MA), PS132

von Seck, P. (Wiesbaden), PS011, PS021

von Stritzky, B. (Berlin), PS144

W

Wächter, L. (Regensburg), PS099 Wagner, M. (Dresden), PS013 Wagner, U. (Leipzig), PS027 Wahl, U. (Halle), PS111 Waizmann, M. (Leipzig), PS165 Waldmann, E. (Erlangen), PS148 Walla, B. (Aachen), PS074 Walter, A. (Hamburg), PS135 Wang, G. (Cambridge, MA), PS132 Watanabe, D. (Nagakute), PS143 Weber, P. (Pforzheim), PS112 Weerawarna, T. (Pforzheim), PS112 Weger, B. (München), PS157 Weibert, E. (Berlin), PS129 Weidemann, F. (Unna), PS003 Weigand, K. (Regensburg), PS082, PS117

Weis, J. (Regensburg), PS150 Welte, T. (Hannover), PS110 Wenisch, S. (Gießen), PS050
Werhahn, S. (Göttingen), YIA04 Werner, J. (Gießen), PS050 Weßling, J. (Münster), PS125 Westhoff, T. (Herne), PS024, PS100 Wex, C. (Magdeburg), PSO30 Wheeler, C. (London), YIA04 Wiedenroth, J. (Homburg), PS033, PS105

Wild, P. (Mainz), YIA05, PS140 Wilke, H. (Essen), PS093 Wilke, K. (Dresden), PS069 Wilke, T. (Wismar), PS155 Will, U. (Gera), PS115 Willecke, F. (Freiburg im Breisgau), PS067, PS068

Winter, S. (Köln), YIA10 Wirtz, H. (Leipzig), PS004 Wirtz, S. (Erlangen), PS056 Wisloff, U. (Trondheim), PS149 Witte, B. (Magdeburg), PS121 Wittmann, F. (Mannheim), PS147 Wöhrle, A. (Esslingen), PS089 Wolf, D., PS067, PS068 Wolff, S. (Magdeburg), PS001 Wollert, K. (Hannover), PS013 Worth, H. (Fürth), PS172 Wullich, B. (Erlangen), PS148 Wurm, S. (Nürnberg), PS164

\section{Y} Yeo, W. (Wollongong, NSW), PS142, PS143

\section{Z}

Zamyatkin, O. (Homburg), PS103 Zawada, A. (Homburg), PS123 Zelzer, S. (Graz), PS130 Zenker, B. (Erlangen), PS052 Zenker, M. (Magdeburg), PS121 Zhu, C. (Uxbridge), PS086, PS098 Ziller, V. (Marburg), PS094 Zimmer, M. (Offenbach), PS173 Zimmermann, C. (Regensburg), PS119

Zirlik, A. (Freiburg), PS067, PS068

Zölch, B. (München), PS096

Zopf, W. (Stuttgart), PS174

Zopf, Y. (Erlangen), PS051, PS052,

PS054, PS056, PS058, PS146, PS148

Zou, J. (Freiburg im Breisgau), PS067 